\title{
Plant defence strategies to multi-herbivore attack
}

Maite Fernández de Bobadilla

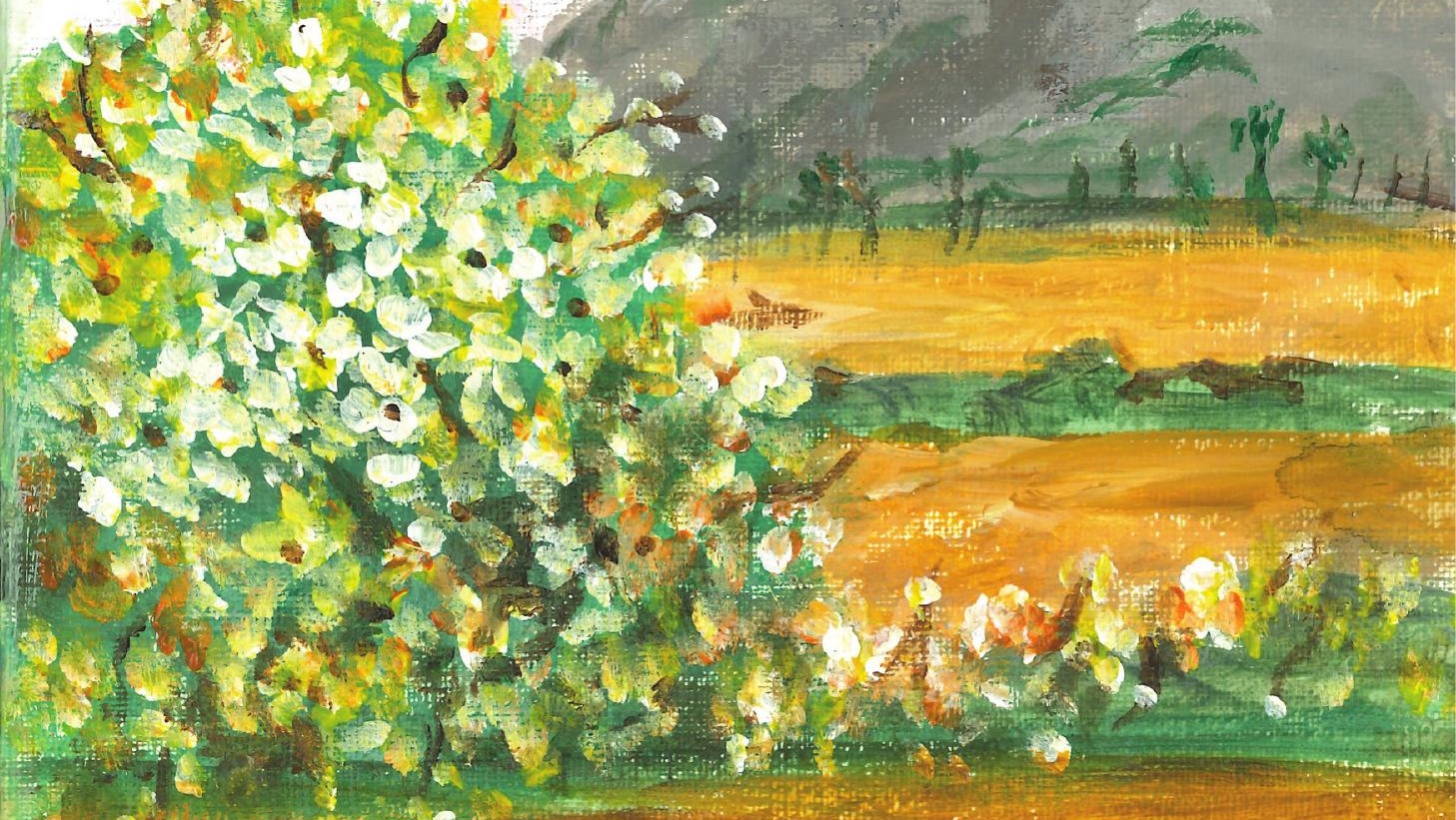

(6)

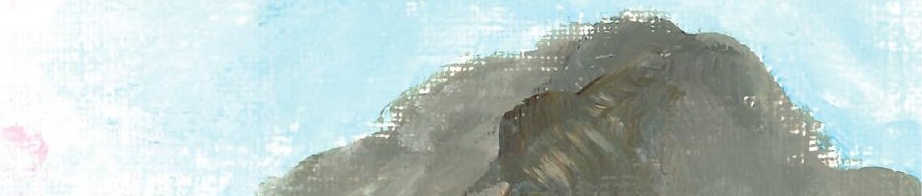

of ${ }^{2}=2$

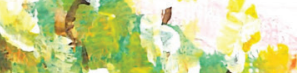




\section{Propositions}

1. Herbivore species richness in multi-herbivore attack on plants influences plant resistance to a subsequent attacker, the plant-associated insect community and plant fitness. (this thesis)

2. Plants tailor their defence strategies to the anticipated arrival of the most prevalent herbivores.

(this thesis)

3. Transferring knowledge to society rather than to the rest of the scientific community is the first priority for scientists.

4. Current measures of scientific success are not representative for scientific quality.

5. Discriminatory behaviours originate from either ignorance or the desire to maintain privileges.

6. Collaboration between people is the way to achieve the Sustainable Development Goals.

Propositions belonging to the thesis entitled 'Plant defence strategies to multi-herbivore attack' Maite Fernández de Bobadilla

Wageningen, 30 June 2021 
Plant defence strategies to multi-herbivore attack

Maite Fernández de Bobadilla 


\section{Thesis committee}

\section{Promotors}

Dr E. H. Poelman

Associate Professor, Laboratory of Entomology

Wageningen University \& Research

Prof. Dr M. Dicke

Professor of Entomology

Wageningen University \& Research

\section{Other members}

Dr Colette Broekgaarden, Keygene, Wageningen

Prof. Dr Francine Govers, Wageningen University \& Research

Dr Sybille Unsicker, Max Planck Institute for Chemical Ecology, Jena, Germany

Dr Klaas Vrieling, Leiden University

This research was conducted under the auspices of the Graduate School Experimental Plant Sciences (EPS) 


\title{
Plant defence strategies to multi-herbivore attack
}

\author{
Maite Fernández de Bobadilla
}

Thesis

submitted in fulfilment of the requirements for the degree of doctor at Wageningen University

by the authority of the Rector Magnificus,

Prof. Dr A.P.J. Mol,

in the presence of the

Thesis Committee appointed by the Academic Board

to be defended in public

on Wednesday 30 June 2021

at 1.30 p.m. in the Aula. 
Maite Fernández de Bobadilla

Plant defence strategies to multi-herbivore attack 284 pages

PhD thesis, Wageningen University, Wageningen, the Netherlands (2021) With references, with summary in English

ISBN: 978-94-6395-796-0

DOI : https://doi.org/10.18174/545962 

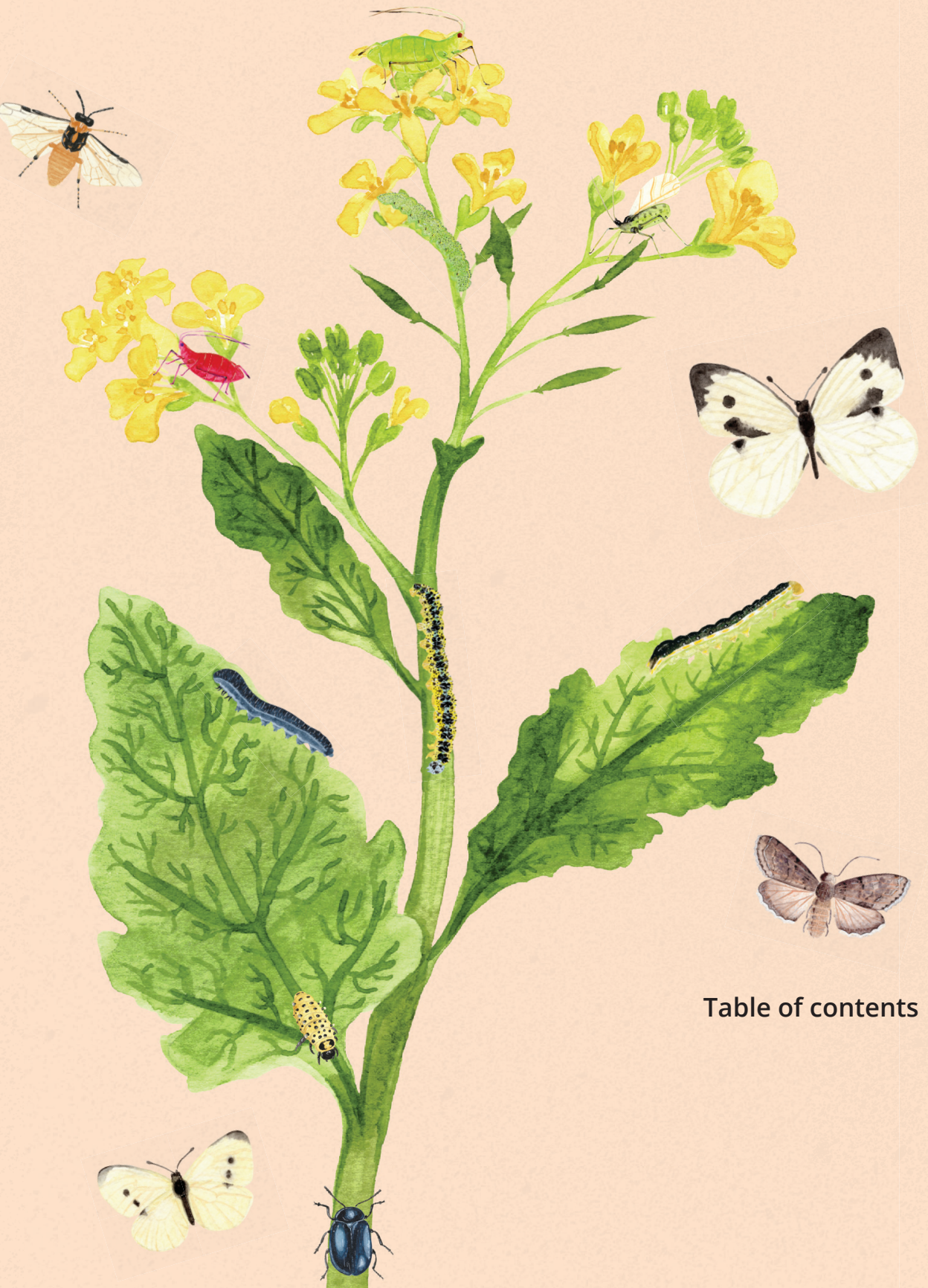



\section{Chapter 1}

General Introduction

9

\section{Chapter 2}

Plant defence strategies to multi-herbivore attack

Maite Fernández de Bobadilla, Alessia Vitiello, Matthias Erb and Erik H. Poelman

\section{Chapter 3}

Plant defence to sequential attack is adapted to prevalent herbivores

Daan Mertens, Maite Fernández de Bobadilla, Quint Rusman, Janneke Bloem, Jacob C. Douma, Erik H. Poelman

\section{Chapter 4}

Insect species richness affects plant responses to multi-herbivore attack

Maite Fernández de Bobadilla, Mitchel E. Bourne, Janneke Bloem, Sarah N. Kalisvaart, Gerrit Gort,

Marcel Dicke, Erik H. Poelman

\section{Chapter 5}

Herbivore species richness influences plant interactions with an herbivorous insect, its natural enemies, and plant reproduction

Maite Fernández de Bobadilla, Quint Rusman, Thibault Costaz, Cristina Alban, Kalliopi Arkoumanea,

Daan Mertens, Erik. H. Poelman

\section{Chapter 6}

Plasticity in induced resistance to sequential attack by multiple herbivores in

Brassica nigra

Maite Fernández de Bobadilla, Roel van Wiechen, Gerrit Gort, Erik. H. Poelman

\section{Chapter 7}

General Discussion

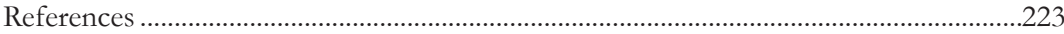

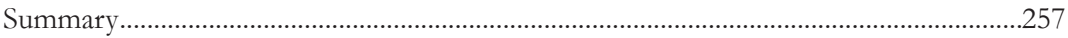

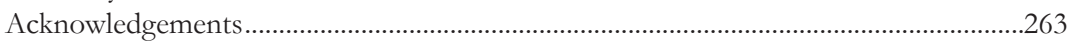

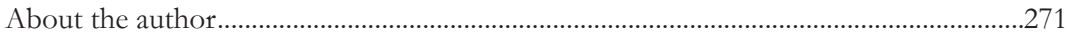

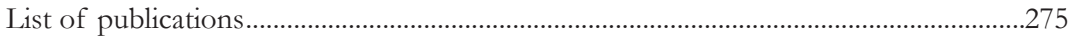

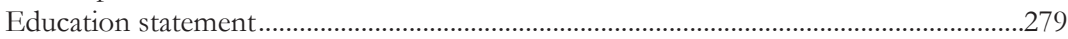




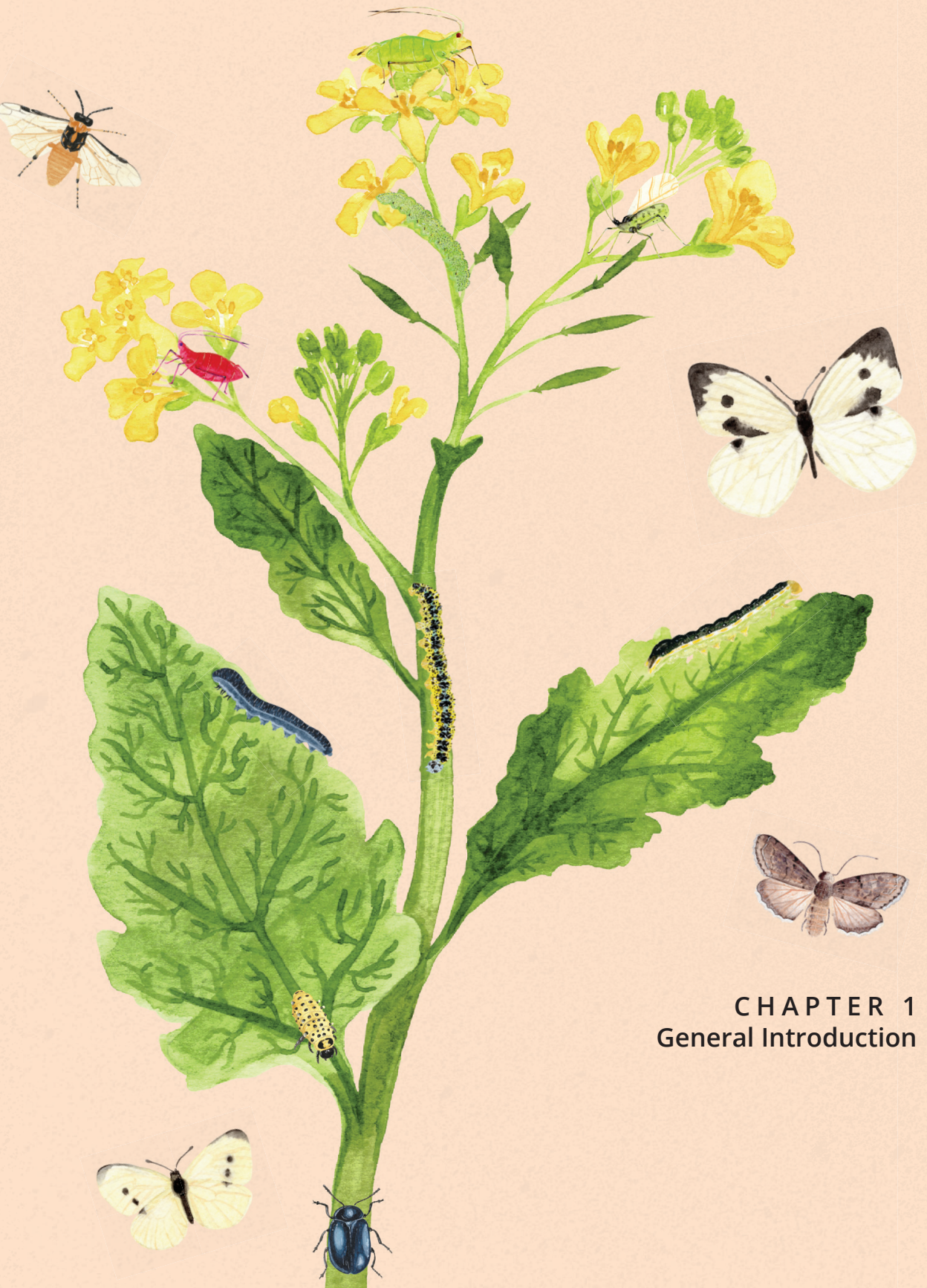



\section{INTRODUCTION}

Plants live in a complex environment where they interact with a community of diverse organisms ranging from microbes to mammals. Insects are very important members of that community of organisms because of the number of interactions and the large effect they have on plant fitness (Cozzolino et al., 2015; Lankau \& Strauss, 2008; Soper Gorden \& Adler, 2018). Some of the interactions with insects are detrimental for the plant such as interactions with herbivores (Grass et al., 2018), whereas others are beneficial such as interactions with natural enemies of the herbivores and with pollinators (Gervasi \& Schiestl, 2017; Ollerton et al., 2011). Herbivores often have a direct negative effect on plant fitness, because they consume plant tissues and reduce the photosynthetic capacity of the plant (Nabity et al., 2009). In contrast, natural enemies of the herbivores, such as parasitoids are hypothesized to have a positive effect on the plant, because they control the populations of the herbivores that consume plant tissues (Romero \& Koricheva, 2011). Additionally, interactions with pollinators have an enormous impact on plant fitness, because the majority of flowering plants depend on pollinators for reproduction (Ollerton et al., 2011). In order to maximise their fitness, plants need to prevent herbivores from eating their tissues while maintaining interactions with beneficial organisms (Grass et al., 2018; Herrera et al., 2002). In nature, plants are attacked by multiple insect herbivores that induce changes in plant phenotypic traits and affect plant interactions with other organisms such as other herbivores, natural enemies of the herbivores and pollinators (Moreira et al., 2019). However, how plants defend themselves against the attack of multiple insects while maintaining the attraction of parasitoids and pollinators in order to maximise fitness, is poorly understood (Dicke et al., 2009).

\section{PLANT DEFENCE AGAINST HERBIVORES}

To protect their tissues from being eaten, plants are equipped with constitutive defence traits that are always present independent of the presence of the attacker. If a herbivore attacks the plant, another layer of defence comes into play and plants change their phenotype by expression of an induced 
defence response, that is tailor made to the current attacker (Karban, 2011). This induced response will directly target the attacker, and aims to reduce the damage the attacker inflicts to the plant, known as direct defence. The induced response will not only directly target the herbivore, but may also attract natural enemies of the herbivore, such as predators and parasitoids, known as indirect defence (Bustos-Segura et al., 2020; Karban et al., 1997). Induced plant resistance to herbivory is modulated by the identity (Agrawal et al., 2014; Van Zandt \& Agrawal, 2004), guild (Bidart-Bouzat \& Kliebenstein, 2011; Davidson-Lowe et al., 2019), and density of the attacker (Kroes et al., 2016; Kroes et al., 2015; Pineda et al., 2017; Ramirez \& Eubanks, 2016), among others. Only recently, studies including more than two attackers have unravelled that the order of attackers also influences a plant's ability to defend against attackers (Stam et al., 2017, 2018, 2019; Viswanathan et al., 2007). Nonetheless, we are far from understanding how plants defend themselves against the attack of more than two herbivores or in other words multi-herbivore attack.

\section{MOLECULAR REGULATION OF PLANTS TO DEAL WITH HERBIVORE ATTACK}

Plants can sense virtually every external stimulus surrounding them (Kliebenstein, 2014). Plant interactions with their biotic environment are regulated in an integrated system, where information travels at the molecular level (gene, transcript, metabolite) and at the morphological level (cells, tissues and the whole plant) (Atkinson \& Urwin, 2012). Irrespective of whether interactions with organisms are beneficial, detrimental or neutral for the plant, plants can detect all of them. Detection is mediated by plant receptors that recognise specific molecules present in the organisms and activate a regulatory response. The molecules that trigger a response in the plant have been named associated molecular patterns (AMPs), and depending on the organism (or organism part) they are called PAMPs, MAMPs, HAMPs, DAMPs or EAMPs when they come from a pathogen, a microorganism, a herbivore, damage or an egg, respectively (Acevedo et al., 2015; Gouhier-Darimont et al., 2019; Jones \& Dangl, 2006; Kliebenstein, 2014). When feeding from the plant, HAMPs and DAMPs come into contact with the inner part of the cell. 
HAMPs include molecules present in the insect oral secretion that elicit a defence response whereas DAMPs are cell fragments mixed with intracellular components. Together the HAMPs and DAMPs resulting from the leaf-chewing process by the herbivore, characterize the signature from chewing insects (Acevedo et al., 2015; Erb \& Reymond, 2019). The different AMPs bind to specific pattern recognition receptors (PRRs) in the plant, which will start a defence response. Several HAMPs have been identified in insect oral secretions (Schmelz et al., 2006; Truitt et al., 2004). However, so far only PRRs for DAMPs have been described (Erb \& Reymond, 2019). Early signalling events triggered by herbivory include 1) depolarization of the plasma transmembrane potential, 2) rise in cytosolic $\mathrm{Ca}^{2+}$; 3) production of reactive oxygen species (ROS) and 4) mitogen activated protein kinase (MAPK) activity. The early signalling activated upon herbivore attack is connected to broad transcriptional rearranging and a defence response regulated by hormonal signalling networks (Maffei et al., 2007).

Despite the specificity in herbivore recognition (especially well-supported in plant-pathogen interactions) the signalling cascade that regulates phenotypic changes induced by herbivore attack seems more general. The Jasmonic Acid (JA) signalling is the core pathway regulating plant defence responses, especially against chewing insects.

JA biosynthesis occurs via the lipoxygenase pathway which is activated upon mechanical damage and herbivory. Upon damage, acyl-lipidic hydrolases release $\alpha$-linolenic acid from the plastid membrane, which is the substrate for the enzyme lipoxygenase (LOX) which activates the expression of LOX genes (Bell et al., 1995).

A key regulator of the first steps in JA biosynthesis is the enzyme lipoxygenase (LOX) which activates expression of JA-biosynthetic genes, such as LOX2 (Bell et al., 1995; Sarde et al., 2018). 12-oxophytodienoic acid (OPDA) is generated and transported to the peroxisome, where it undergoes three $\beta$-oxidation steps and forms (+)-iso-JA that is transported to the cytosol and 
conjugates with isoleucine. JA-isoleucine (JA-Ile) is the bioactive form of JA that is transported to the nucleus where it binds to nuclear receptors, formed by CORONATINE-INSENSITIVE1 (COI1), JASMONATE-ZIM DOMAIN (JAZ), and an inositol-polyphosphate cofactor. The binding of JA-Ile to JAZ triggers JAZ degradation by the proteasome, which in turn releases the repression of MYC transcription factors, resulting in expression of JA-responsive genes, such as $\boldsymbol{V S P 2}$, which are involved in expression of defence genes against a wide diversity of herbivores (Erb \& Reymond, 2019). Salicylic acid (SA), ethylene (ET) and abscisic acid (ABA) are well-known stress-related phytohormones that are also induced upon herbivory and are modulators of the defence response. Moreover, growth hormones, such as gibberellins (GAs), auxin and cytokinins (CKs) are involved in antiherbivore responses. GAs regulate growth via proteasome-mediated degradation of DELLA repressors, which modulate the JA pathway. Activation of the GA pathway leads to DELLA degradation and inhibition of JA responses (Erb \& Reymond, 2019).

In general, plant-induced responses to attack by insects of the chewing feeding guild are regulated via the JA-phytohormonal pathway whereas plant responses to attack by phloem feeders are regulated via the SA-pathway (Erb et al., 2012; Jaouannet et al., 2014; Züst \& Agrawal, 2016). Deficiency in SA makes tomato plants more susceptible to Myzus persicae whereas SA-deficient Arabidopsis plants are not affected in resistance (Erb \& Reymond, 2019) and mutants in the JA biosynthetic pathway generally show reduced resistance against chewers (Ye et al., 2012). The different signalling pathways interact and can stimulate or inhibit each other. The best described example is the negative crosstalk between SA and JA. Several plant species that have been attacked by phloem-feeding insects cannot defend against a chewer as well as undamaged plants do (Moreira et al., 2018; Pieterse et al., 2012; Soler et al., 2012). In addition, ET/JA and ABA/ ET antagonism has also been reported, and this further modulates plant defence against herbivores (Verhage et al., 2011). Additionally, transcription factors play a key role in regulating defences both up- and downstream of the phytohormone signalling. Downstream of phytohormonal signal-transduction, induced plant 
secondary metabolites can regulate plant defence. For example, benzoxazinoids in maize induce callose deposition (Kliebenstein, 2014).

Herbivore-induced plant phenotypic changes start at the damaged site (Chandran et al., 2010). At the cell level, cell autonomous responses to the organism will be first transported to neighbouring cells, to the whole leaf, and to the whole plant to form a systemic response (i.e. in distal plant tissues). The communication in plants is not limited to a systemic signal within one individual. Plants interact and communicate with their neighbouring plants via volatiles and via the roots, exchanging information useful to survive, such as the presence of danger (Babikova et al., 2013; Farmer \& Ryan, 1990). Importantly, there is also interkingdom communication between plants and micro- and macro-organisms. Striking examples of interkingdom communication are plants detecting and discriminating fungal volatiles from different soil-borne fungi, and modulating root growth direction (Moisan et al., 2021) or parasitoids differentiating volatiles from plants attacked by their host from undamaged plants or plants attacked by a different herbivore (De Rijk et al., 2016a,b). Herbivory induces dramatic changes in a plant phenotype, and because plants communicate and interact with a whole range of organisms, it is not surprising that herbivory alters the dynamics of the plant-associated community (Pashalidou et al., 2015; Soper Gorden \& Adler, 2018; Stam et al., 2014).

\section{HERBIVORE INDUCED CHANGES ON THE INSECT COMMUNITY}

The induced response against one herbivore does not only affect the current attacker, but may also affect the attraction and the performance of other insects that colonise the plant later including other herbivores, natural enemies of the herbivores and pollinators (Cozzolino et al., 2015; Ohgushi, 2008; Rusman et al., 2018; Van Zandt \& Agrawal, 2004). As all these community members may have strong effects on plant fitness, this change in interactions can have dramatic effects for the plant (Cozzolino et al., 2015; Glaum \& Kessler, 2017; Kessler \& Chautá, 2020; Soper Gorden \& Adler, 2018). Consequently, plant 
responses to herbivory can alter community dynamics and plant trait selection (Lankau \& Strauss, 2008; Strauss, 2014; Strauss \& Irwin, 2004).

Parasitoids are generally hymenopteran or dipteran species that predate on other insects, and therefore are classified as natural enemies of the herbivores. The foraging of parasitoids is highly affected by herbivore-induced changes in a plant phenotype. Upon attack by insect herbivores, plants release herbivoreinduced plant volatiles that attract parasitoids, acting as a "cry for help" (Dicke \& Baldwin, 2010). Parasitoids are able to discriminate between plants that have been attacked or not, and even on the type and density of attackers (De Boer et al., 2008; De Rijk et al., 2013, 2016a-c). In nature, parasitoids need to find their host in a complex environment, where some plants are attacked by hosts, others by non-host herbivores and yet others may even be attacked by several attackers (Aartsma et al., 2017, 2019). We know that parasitoids are able to discriminate between plants under single or dual attack (Blubaugh et al., 2018; Ponzio et al., 2016; Rodriguez-Saona et al., 2003, 2005), however, we lack studies investigating whether parasitoid foraging is affected by multiple attack. This knowledge will help us better understand parasitoid foraging under more natural conditions.

Importantly, plant defence against herbivores may trade off with pollinator attraction. Herbivore-induced changes in the volatile blend of the plant can also affect attraction of pollinators. Additionally, herbivory induces changes in important cues for pollinator recruitment such as flower colour/ shape/odour, pollen and nectar quality (Lucas-Barbosa, 2016; Lucas-Barbosa et al., 2016; Nicholls \& Hempel de Ibarra, 2017; Rusman et al., 2018, 2019 a,b). Field studies show that the community of pollinators of B. nigra plants changes when the plants are under single herbivore attack early in the season. Interestingly, plant-mediated interactions between herbivores and pollinators are different depending on the species of herbivore attacking the plant (Rusman et al., 2018). These studies show that plant responses to single herbivore attack can affect pollinator attraction, but we lack information whether the response of the pollinators is affected by multiple herbivore attack. 


\section{GENERAL OBJECTIVE AND RESEARCH QUESTIONS OF THIS THESIS}

The aim of my PhD project, was to explore how Brassica nigra plants deal with multiple attack by its most common herbivores. In my thesis I explored four main questions (Fig. 1):

1) Can plants anticipate arrival of the most prevalent herbivores and tailor their induced defence strategies accordingly? (chapter 3)

2) Does species richness of previous attackers affect a plant's capability to respond to a subsequent attacker of the same or of a different feeding guild? (chapter 4)

3) Does species richness of previous attackers affect plant interactions with herbivores, parasitoids, pollinators and do these effects result into plant fitness consequences in the field? (chapter 5)

4) Are plants able to defend against a late arriving herbivore after a history of sequential attack by four other herbivores? (chapter 6)

\section{STUDY SYSTEM}

\section{The black mustard, Brassica nigra}

As a focal plant I used the wild annual plant Brassica nigra (Brassicaceae family). This pretty plant contains yellow flowers, is a fast growing species, highly branched, and an obligatory outcrossing species. Its leaves and flowers contain glucosinolates, secondary metabolites characteristic for the Brassicaceae involved in defence against herbivores and attraction of pollinators. The extensive information on the ecology and mechanistic machinery of B. nigra available makes B. nigra an excellent focal plant to address the main questions of my PhD. First, the herbivore community of B. nigra plants is well characterised (Mertens et al., 2020 thesis chapters 3,6; Mertens et al., 2021; Poelman et al., 2009). Additionally, some studies addressed herbivore-induced responses to single attack in B. nigra (Van Dam et al., 2005; Van Dam \& Raaijmakers, 2006). There is even modelling exercises made to assess the plant growth defence trade-off under conditions of herbivory and competition for light (de Vries et al., 2017, 2018, 2019). Second, the pollinator community of B. nigra plants, as well as the effects of single attack by 


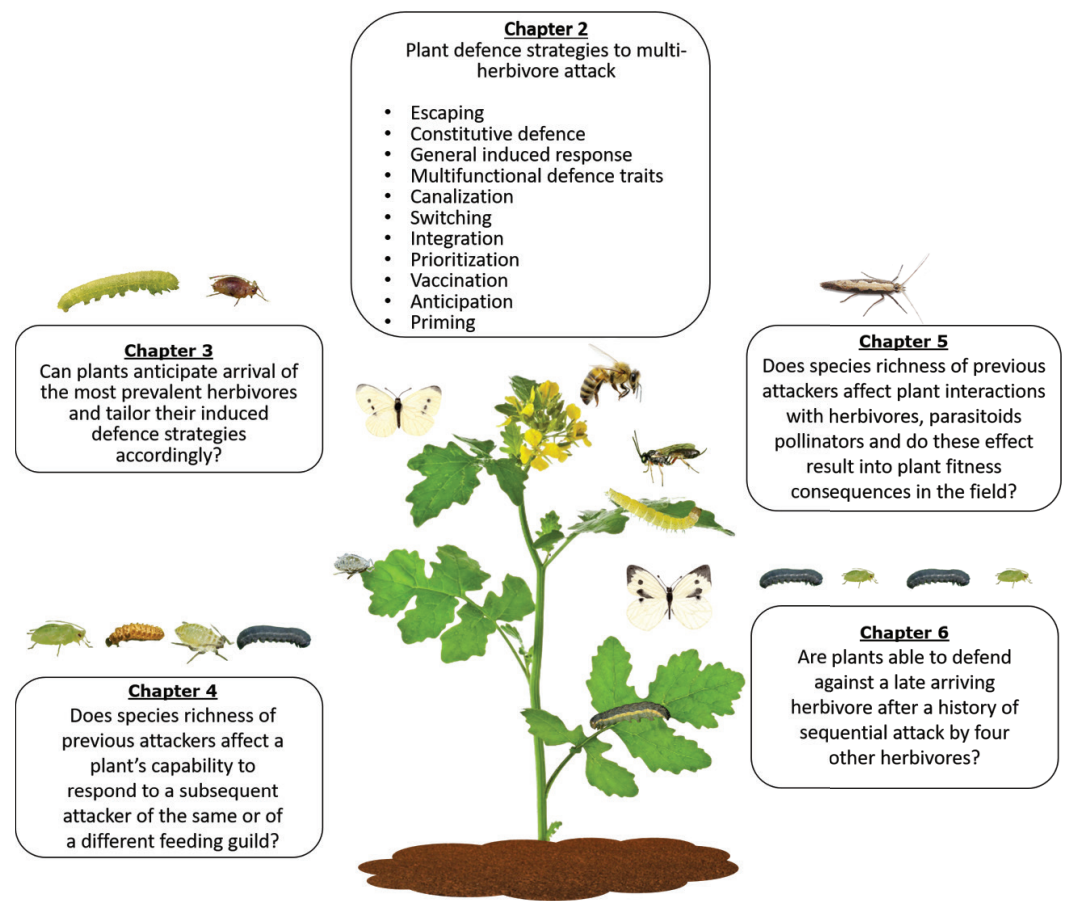

Figure 1. Overview of the main questions investigated in each chapter of the thesis. 
different herbivores and the effects of dual attack on the pollinator community have also been explored (Chrétien et al., 2021; Rusman et al., 2018, 2020). Third, there is information on how community complexity shapes selection on traits in $B$. nigra (Lankau \& Strauss, 2008). Fourth, other studies have addressed mechanistic and molecular interactions of B. nigra with several stressors. These studies include transcriptomic responses to single attack by insects of different feeding guilds (Broekgaarden et al., 2011), metabolic and phytohormonal profile after dual attack (Chrétien et al., 2018; Papazian et al., 2019; Ponzio et al., 2017), and transcriptomic responses to dual attack (Bonnet et al., 2017). Even recently, the genome of B. nigra has been sequenced, allowing for a more in-depth study of the molecular regulation of this plant's responses to interactions with members of the associated community (Perumal et al., 2020). Thus, the ecological and molecular knowledge basis of B. nigra plants, allowed me to design ecologically relevant experiments related to the plant's responses to multi-herbivore attack.

\section{Insect herbivores used in the experiments}

To study plant adaptations to the attack by multiple insect herbivores, I challenged my plants with 11 insect herbivores (Table 1). I applied herbivores with a chewing feeding guild and with a phloem feeding guild. All the herbivores I used in my experiments are commonly found in the field attacking B. nigra plants (Mertens et al., 2021) and therefore may be important selection agents on this plant species (Table 1).

\section{Chewers}

As chewing insects, I used seven insect species, including larvae of Lepidoptera, Coleoptera and Hymenoptera. Among them there were two generalist species and five species specialized on brassicaceous plants.

The silver Y Autographa gamma, and the cabbage moth Mamestra brassicae (both Lepidoptera: Noctuidae) are generalist insects, their larvae are polyphagous and attack Brassicaceae plants but also Leguminosae, Solanaceae and many other plants. 


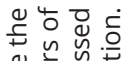

ข ㄴํำ

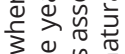

ज过 है

듬

$\frac{2}{\sigma} \sqsubseteq \frac{1}{1}$

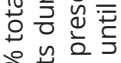

อ兄艺

은 응 등

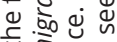

‡

ง

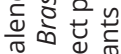

む)

능ㄴㅇ 흔

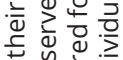

은응으은

ㅁํำ 등

언웡 는

๘

는 는 음

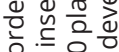

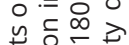

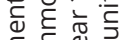

ह ह ญ ह

ญ는

증ํํ

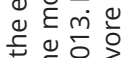

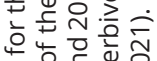

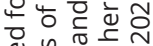

ปับํำ

ฆ ్ㅓㅇㅠ 워

이는

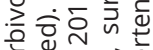

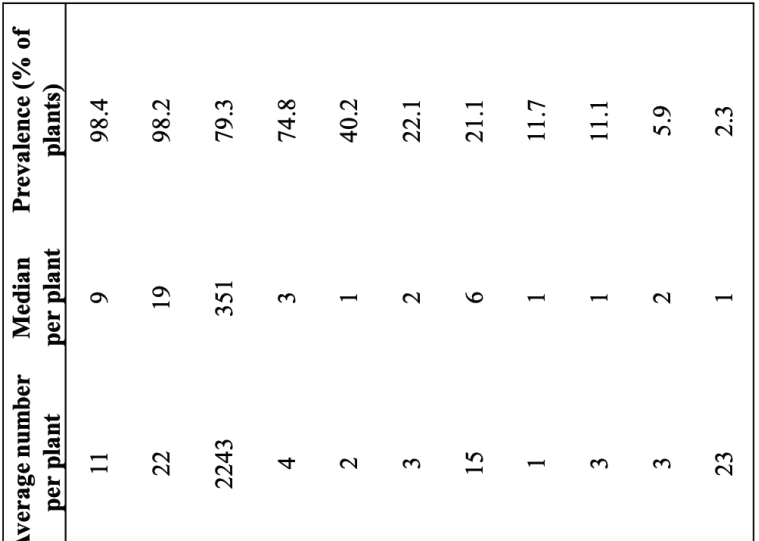

选苍点这

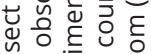

드 는 는

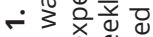

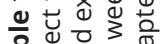

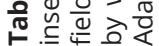

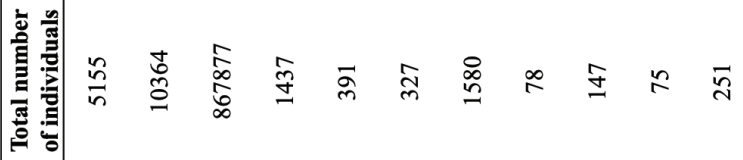

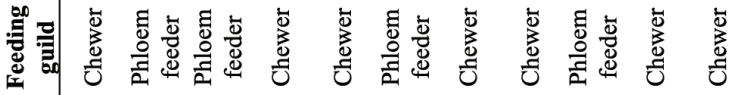

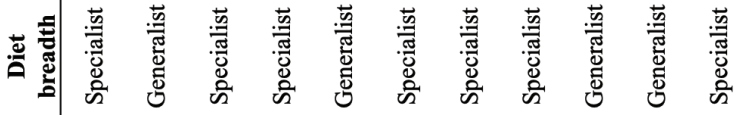

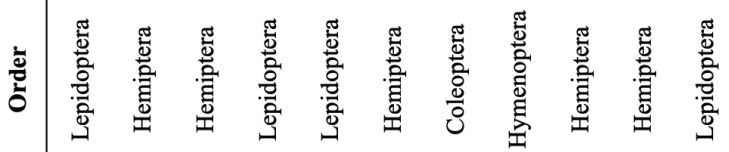

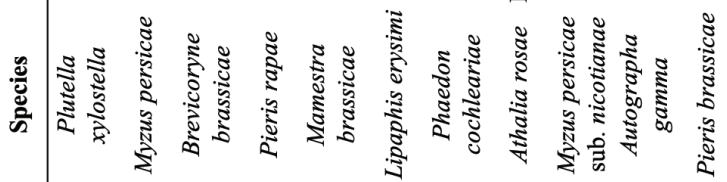

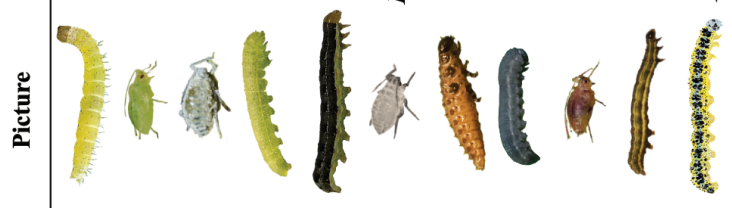


The turnip sawfly Athalia rosae, (Hymenoptera: Tenthredinidae) is a specialist herbivore of brassicaceous plants. Female wasps lay eggs on the leaf edge and upon hatching larvae feed voraciously until they consume all the leaf tissue and move to another leaf. Larvae and adults of the mustard leaf beetle Phaedon cocbleariae (Coleoptera: Chrysomelidae) are herbivores specialised on brassicaceous plants. Adults emerge in large numbers, and due to the high mobility and voracious feeding they can devastate fields of Brassicaceae plants. When larvae have acquired enough body mass, they burry themselves in the soil for pupation. Butterflies of the large white Pieris brassicae (Lepidoptera: Pieridae) lay eggs in clutches on the leaf surface of Brassicaceae plants (Fatouros et al., 2015). Upon hatching, the larvae feed gregariously from that same leaf and when they reach the second instar they move to the buds and flowers. Butterflies of the small cabbage white Pieris rapae (Lepidoptera: Pieridae) lay single eggs on brassicaceous plants and larvae feed from leaves. The diamondback moth Plutella xylostella (Plutellidae) is a specialist insect of plants of the Brassicaceae family with a short generation time and high fecundity. Larvae of P. xylostella cause big economic losses worldwide. Female moths of P. xylostella are attracted to glucosinolate breakdown products and lay many eggs on brassicaceous plants. Upon hatching, larvae burrow a mine inside the leaf, and after a few days they leave the mine and start chewing the whole leaf.

\section{Phloem-feeding insects}

As phloem-feeding insects, I used four aphid species, all belonging to the Hemiptera (Aphididae). The cabbage aphid Brevicoryne brassicae is a specialist of brassicaceous plants; they can reach big colonies of thousands of individuals on leaves, stems, buds and flowers. The mustard turnip aphid, Lipaphis erysimi, is also a specialist insect that can be found mostly on lower stems, but also on leaves and flowers of brassicaceous plants. The green peach aphid, Myzus persicae is a very generalist phloem feeder, that can be found on more than 400 plant species, including brassicaceous plants. The red tobacco aphid, Myzus persicae sub. nicotianae is an important pest on tobacco cultivars, although it feeds from many other plant species, including B. nigra. 


\section{OUTLINE OF THIS THESIS}

In chapter 2, I propose a framework for plant defence to multi-herbivore attack by defining the repertoire of plastic defence strategies that may allow plants to optimize defence against multiple herbivore attack. These defence strategies could be seen as risk management tools to deal with a multitude of stressors.

In Chapter 3, I investigated whether the level of induced resistance to sequential herbivory in B. nigra corresponds with the likelihood of the order and nature of sequential insect attack in the field. I linked observations of the order of arrival of insect herbivores during three years of season-long insect community assessment on 488 plants, to a full-factorial assessment of induced resistance in pairwise plant-mediated interactions between ten herbivore species. I specifically tested whether induced resistance of the second herbivore can be predicted by traits (feeding guild and diet breadth) of the inducing herbivore or the secondary receiving herbivore species, as well as the commonality of those species and their pairwise interactions in the field (Mertens et al., 2021). Moreover, to characterise the specificity of induced plant response, I studied the expression of the JA-biosynthesis marker gene (LOX2) and of the SAresponsive gene (PR1) after plants were attacked by the ten herbivores.

In chapter 4, I investigated whether species richness of previous attackers affects a plant's capability to respond to a subsequent attacker of the same or of a different feeding guild. I challenged B. nigra plants with 51 treatments representing attack by an increasing species richness (1,2 or 4 species) of either phloem feeders, leaf chewers, or a mix of both feeding guilds when keeping total density of attackers constant. I selected eight of the most prevalent herbivore species equally distributed across the leaf chewer and phloem feeder guilds and studied how plant responses to these attackers affect resistance to subsequent attack by caterpillars of P. xylostella. Additionally, to characterise the specificity of the induced plant responses to the 51 herbivore combinations I studied the expression of the JA-biosynthesis marker gene (LOX2), of the JA-responsive gene (VSP2), of the SA- biosynthesis gene (ICS) and of the SA-responsive gene (PR1) 48 and $96 \mathrm{~h}$ after herbivore attack. 
In chapter 5, I studied how herbivore species richness and its trait composition affect plant interactions with its insect community and whether these interactions affect plant fitness. I conducted a manipulative field study where I subjected B. nigra plants to attack by an insect herbivore community with increasing species richness from one and/or two feeding guilds (i.e. phloem feeders, leaf chewers or a mix of both). I specifically studied how the composition of the introduced herbivore community affected 1) plant colonisation by the herbivore P. xylostella, 2) top-down control of this herbivore's population by parasitoids, 3) visitation of flowers by pollinators, and 4) how these interactions collectively affect plant fitness in terms of seed production. I examined the relations between the different community members and effects of varying levels of herbivore richness and feeding guild by applying structural equation modelling (SEM). This allowed to partition the direct and indirect effects of different community members on plant fitness, and how early-season herbivore attack alters these relationships.

In chapter 6 , I investigated whether $B$. nigra plants are able to defend themselves against caterpillars of the late-arriving herbivore $P$. xylostella, when plants had been previously exposed to sequential attack by four other herbivores. I manipulated the order of arrival and the history of attack by four herbivores to investigate which patterns in sequential herbivory determine resistance against the fifth attacker. I specifically studied the effect of: 1) the identity and the guild of the first attacker; 2) the identity and the guild of the last attacker; 3 ) the order of arrival of herbivores in a sequence 4) the number of times a plant is attacked by herbivores of the same feeding guild.

Finally, in chapter 7, I summarize and discuss the main contributions of my $\mathrm{PhD}$ to the field of insect-plant interactions. Furthermore, I give a perspective for future research.

ACKNOWLEDGEMENTS I thank Erik Poelman and Marcel Dicke for helpful comments on an earlier version of this chapter. 


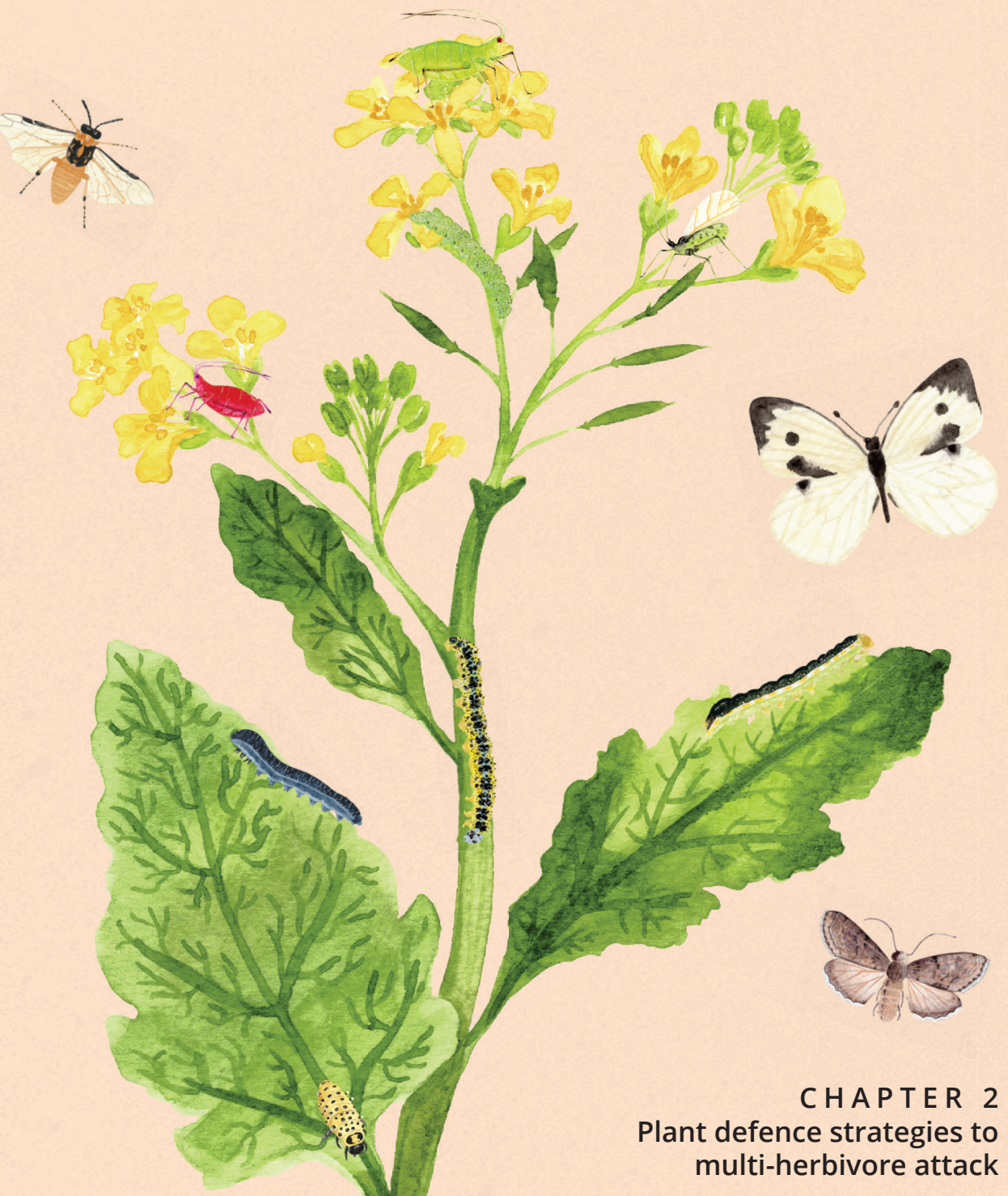

Maite Fernández de Bobadilla ${ }^{a}$, Alessia Vitielloa, Matthias Erba and Erik H. Poelman ${ }^{a}$ a authors contributed equally 



\section{HIGHLIGHTS}

- Virtually all plants are under attack by a species rich community of antagonists. By recognizing the individual attacker, plants may effectively tailor their defence response. However, the response to one attacker should be balanced with the costs that this response may entail towards defence against other attackers.

- Reprogramming of plant physiology to counteract herbivory is typically studied under single or at most dual attack. Often these studies identify antagonistic interactions that limit plants in their ability to resist a second attacker. The emerging physiological concepts may mismatch with the adaptive nature of plant responses in community context.

- Identifying plant plasticity to deal with their full community of attackers is key in understanding plant defence strategies and requires unified terminology to illustrate the repertoire of plant plasticity matching ecological patterns of attack.

\section{ABSTRACT}

Plants may effectively tailor defences by recognizing their attackers and reprogramming their physiology. Although most plants are under attack by a large diversity of herbivores, surprisingly little is known about the physiological capabilities of plants to deal with multi-herbivore attack. Studies on dual herbivore attack identified that defence to one attacker may cause energetic and physiological constraints to deal with a second attacker. How these constraints shape plant plasticity in defence to multi-herbivore attack and what defence strategies plants may deploy to their full community of attackers is a major knowledge gap in plant science. Here we provide a framework for plant defence to multi-herbivore attack by defining the repertoire of plastic defence strategies that may allow plants to optimize defence against a multitude of stressors. 


\section{PLANTS ARE UNDER SELECTION TO DEFEND AGAINST MULTI-HERBIVORE ATTACK}

Plants interact with a large diversity of organisms in antagonistic or mutualistic relationships. For many plant species, insect herbivores are some of the most prominent and diverse antagonists that often have a direct or indirect negative impact on plant fitness (see Glossary) (Atkinson \& Urwin, 2012). To maximise their fitness, plants use tolerance and resistance strategies (Schuman \& Baldwin, 2016; Erb, 2018). An important asset of the defence repertoire in plants is that they can recognize the specific attacker (Acevedo et al., 2015; Erb and Reymond, 2019; Züst \& Agrawal, 2016) and mount induced responses to tailor resistance to the type of attacker. Such induced responses are likely a cost saving strategy (Karban, 2020). Typically, responses of plants to herbivores are studied by isolating responses to individual stressors. These studies yielded extensive knowledge on plant perception, recognition and regulation of responses to insect attack (Box 1). Over the past decades, studies on dual herbivore attack uncovered interactions between gene regulatory networks involved in response to individual stressors, resulting in distinct responses to multiple attackers (Voelckel \& Baldwin, 2004; Vos et al., 2015; Zarate et al., 2007; Zhang et al., 2009). Importantly, these studies also identified that antagonistic cross communication between these networks may impair plants in maximising resistance to individual stressors, may allow herbivores to exploit the cross communication to suppress plant defence and may result in susceptibility to herbivory (Caarls et al., 2015; Li et al., 2015; Li et al., 2018; Proietti et al., 2018; Thaler et al., 2012). While these results represent a step forward to understand defence responses in a herbivore community context, they do not yet capture the full dynamics occurring in nature, where plants are simultaneously or sequentially attacked by a multitude of organisms (Stam et al., 2014). In theory, plants should optimise their defence strategies to deal with communities of attackers (Mertens et al., 2021; Orrock et al., 2015; Poelman \& Kessler, 2016). However, clear examples and a framework for plant defence optimization towards herbivore communities from a physiological perspective are currently missing. Here, we provide a framework to understand plant adaptive responses to multi-herbivore attack. 


\section{GLOSSARY}

Allocation costs: reductions in growth or reproduction as a direct consequence of diversion of limiting resources to defence processes

Defence costs: cost associated to the expression of defence traits or to the cost of expressing the traits in the absence of plant enemies: allocation, ecological or physiological costs, decreases in fitness resulting from interactions with other species.

Dynamic attacker community: multiple attackers can arrive simultaneously, sequentially, and on high and low numbers.

Ecological costs: costs of plant's defence associated with changes in interactions with community members.

Induced response: defence trait that is activated upon herbivore attack and recognition.

Metabolic costs: energy and resources required for the production and maintenance of defences.

Plant defence trait: plant trait that increases the fitness of plants upon herbivore attack

Plant fitness: contribution of an individual plant to the gene pool of the next generation

Tolerance: ability of a plant to reduce fitness losses of herbivory without preventing the attack.

\section{PLASTICITY TO MATCH COMMUNITY CONTEXT}

From an adaptive point of view, plants should not only balance their investment in defence with maintaining competitiveness for light and nutrients, they should tailor their responses to maximize fitness upon herbivore attack (Ballaré et al., 2019; de Vries et al., 2017; Züst \& Agrawal, 2017). As plants are attacked by communities of herbivores, this implies that plant responses should be balancing the costs and benefits of their responses in a community context (Mertens et al., 2021a,b; Poelman \& Kessler, 2016). Thus, defence 
strategies may be advanced risk management strategies that are analogous to chess games to deal with a multitude of stressors (Mertens et al., 2021b; Orrock et al., 2015).

\section{Specific or generalized responses to herbivory}

When considering that plants will be attacked by a multitude of herbivores, it should be questioned to what extent plants recognise and respond to specific attackers or that the responses target a broader range of multiple attackers (Erb et al., 2012). There is substantial evidence that plants recognize herbivore feeding guilds by their type of damage (piercing-sucking or leaf chewing), specific herbivores by associated elicitors and that plants differentially respond to individual herbivores (Schuman \& Baldwin, 2016; Acevedo et al., 2015; Erb \& Reymond, 2019) (Box 1). However, there is also considerable overlap in core transcription profiles after herbivore attack and little evidence that the expressed phenotype specifically targets the current attacker (Mertens et al., 2021a). Limited specificity may be particularly adaptive under high and predictable herbivore pressure. When herbivore pressure is high, plants express their full defences constitutively to reduce herbivory at high maintenance costs that may have a negative impact on plant growth and fitness (Züst \& Agrawal, 2017) (Box 2). To reduce maintenance costs under high and predictable herbivore pressure, plants may use a general induced response that targets all potential attackers at once. Several studies have shown that individual herbivores induce similar suits of defence compounds, where activity of individual compounds may only effectively target a single herbivore species (Adio et al., 2011; Gonzales-Vigil et al., 2011). This suggests that either these plant species were not capable of recognizing the specific attacker and thus mount a broad spectrum of defences, or that individual plants are predictably attacked by most of the herbivore species in the pool of potential attackers (Mertens et al., 2021b). The costs associated with mounting a broad palette of defence compounds may be reduced when compounds are highly multifunctional and are involved in direct and indirect defence as well as in primary metabolism (Neilson et al., 2013; Quintana-Rodríguez et al., 2018). 


\section{BOX 1: Plant perception, recognition and response to herbivores}

To deal with multiple attackers, that may all require different defensive traits, plants have evolved mechanisms to recognize the specific attacker they are facing (Acevedo et al., 2015; Schuman \& Baldwin, 2016). Plants perceive herbivory via binding and recognition of specific molecules. These molecules may originate from disrupted cell walls that provide general information on plant damage (damageassociated molecular patterns, DAMPS) or provide more specific information on herbivore identity by herbivore-associated molecular patterns (HAMPs) in herbivore saliva for example. Perception and recognition of specific attackers results in mobilisation of resistance through phytohormonal signalling and broad transcriptional reprogramming (Acevedo et al., 2015; Erb \& Reymond, 2019; Schuman \& Baldwin, 2016). Each attacker elicits a unique signature of responses involving a large number of phytohormones. In general, responses to leaf-chewing herbivores such as caterpillars are predominately mediated by Jasmonic Acid (JA), Abscisic Acid (ABA) and Ethylene (ET). Phloem-feeding herbivores such as aphids or biotrophic pathogens typically induce responses that are mediated by Salicylic Acid (SA) induction (Erb \& Reymond, 2019; Erb et al., 2012).

Crosstalk between signal-transduction pathways allows plants to fine-tune responses to multiple attackers (Thaler et al., 2012; Vos et al., 2015). The JA/ ET pathway is activated by JASMONATE ZIM-DOMAIN (JAZ) proteins that regulate JA activity and thereby transcriptional activators. The JA pathway has two branches, the MYC2 branch activates wound-induced responses through transcription of LOX. The ERF branch is connected to JA and ET signalling and activates transcription of PDF1.2 that is primarily involved in responses to necrotrophic pathogens (Hickman et al., 2017). Signalling through one branch has antagonistic effects on the other branch. SA accumulates after attack by sapsucking herbivores and regulates NPR1 and its downstream WRKY and TGA transcription factors. These activate defence gene expression such as those that encode for pathogenesis related proteins (PR1). Crosstalk between the signaltransduction pathways is strongly regulated by the NPR1 proteins and WRKY transcription factors that link the JA and SA pathways (Koorneef and Pieterse, 2008). Signal transduction in the SA pathway has antagonistic effects on JA regulation (Caarls et al., 2015). This may result in aphid-induced susceptibility to caterpillar attack (Ali \& Agrawal, 2014; Soler et al., 2012). 
When it is highly unpredictable when, by which herbivore species and in which order attack will take place, adaptive plant defence strategies may include specific responses to an attacker (Karban, 2020). Theoretically, a more specific response maximizes resistance to the current attacker and reduces investment costs to only the specific defences needed (Karban, 2020). However, a specific response to one herbivore may impair plants in maximising resistance or even result in susceptibility to other herbivores thus compromising fitness. Plant regulation of responses to attack by different herbivore feeding guilds is involved in antagonistic cross communication by which aphids may reduce plant resistance to caterpillars (Ali \& Agrawal, 2014; Fernández de Bobadilla et al., 2021; Soler et al., 2012; Thaler et al., 2012). Also, investment in plant family specific defence chemistry may be effective against generalists, but ineffective against specialists (Ali \& Agrawal, 2012). Recent studies further identify that cross-resistance or susceptibility via induced responses may be highly specific for the interacting herbivore species pair (Mertens et al., 2021a; Moreira et al., 2018). How specificity in response to individual herbivores and physiological constraints of dealing with a multitude of attackers is reflected in plant defence strategies is poorly defined and explored.

\section{Defining plastic defence strategies to match multi- herbivore attack}

Here we define the repertoire of plastic defence strategies that may allow plants to use specific responses to individual attackers under a multitude of stressors interacting with the plant (Box 2). A strong specific induced response to a first attacker may limit the potential to respond to other stressors. Several plants show such canalization in their response and cannot deploy a full response to subsequent stressors (Viswanathan et al., 2007). The reduced potential to respond to a second attacker may come from prioritizing defence to the first attacker over growth, which reduces energetic potential to mount defences against subsequent attackers (Li et al., 2015; Li et al., 2018). Antagonistic cross-communication between signal transduction pathways may impair resistance to caterpillars after responding to aphid attack (Ali \& 
Agrawal, 2014; Fernández de Bobadilla et al., 2021; Soler et al., 2012; Thaler et al., 2012). The canalization strategy may be adaptive if the first attacker is the most damaging, or if the phenotype in response to this first attacker has broad cross-resistance to subsequent attackers (Adio et al., 2011; Gonzales-Vigil et al., 2011). In these scenarios, plants may save costs of defence since they do not need to invest energy in redirecting resources towards every new stressor. This strategy may not be the optimal response during different ontogenetic stages, or in different plant organs, because it may lead to reduced potential to deal with subsequent attackers or negatively affect interactions with beneficial organisms (Rusman et al., 2019; Thaler et al., 2012). To deal with some of these costs, the strategy can be modified by adopting tissue specific canalization where each plant organ particularly responds to the first attacker (Wolinska et al., 2019).

Plants may play a switching strategy if resistance to each herbivore requires very different responses, and the costs of switching phenotype are low. The costs not only entail metabolic investment in the new resistance traits, but if the former attacker is still present the switch will lead to vulnerability to the first attacker (Heil, 2010). Thus, completely switching defence phenotype may only be advantageous when multiple herbivores arrive at different moments on the host plant (Barton \& Koricheva, 2010; Ochoa-López et al., 2020), or in herbivorespecies-poor communities (e.g. high altitude or very dry ecosystems) where it is very rare that two herbivores arrive at the same time. The metabolic costs of switching may be reduced when biosynthetic enzymes and intermediates are shared between pathways that regulate resistance to each individual attacker ( $\mathrm{Li}$ et al., 2018), for example through remobilization of resources from secondary to primary metabolisms and vice versa or reabsorbing secondary metabolites to synthesize new chemicals needed for a different response. The switching strategy also implies that plants may reconfigure gene networks involved in crosstalk between pathways to avoid antagonistic crosstalk or switch resistance downstream by for example converting secondary chemistry into different 
components matching the type of attacker or by resource allocation strategies (Li et al., 2015; Li et al., 2018).

Many plant species find themselves in ecological settings where stressors occur simultaneously including the interaction with several different herbivore species at the same time (Stam et al., 2014). These situations require plants to play a strategy of integrating defence responses to all antagonists that may be regulated through crosstalk between signal-transduction pathways. The plant phenotype thus compromises resistance potential to each individual attacker, but yields a net benefit of resistance in perspective of the full attacker community Davidson-Lowe et al., 2019; Eisenring et al., 2018; Mathur et al., 2013; Stam et al., 2017). The costs of an integrated response may arise when plants are not able to effectively deal with the most ravaging herbivore species in terms of their effect on plant fitness. Such costs are likely to be countered by prioritizing responses to the most damaging herbivores. Thus, plants may integrate responses to multiple attackers, but upon attack by a key herbivore redirect their resistance to particularly deal with that attacker. The costs arising from prioritizing resistance to one attacker is suboptimal resistance against other attackers and thus such strategies are likely to only arise when fitness costs of attack by herbivore species substantially differ. Under these conditions we may also find plants to strongly respond to particular herbivore species that themselves may not inflict large fitness costs, but with their presence reduce the likelihood that the plant will be colonized by more ravaging herbivores (Poelman \& Kessler, 2016). A response to one herbivore may vaccinate the plant to arrival of other herbivores and such situations may lead to plants even acquiring some herbivores that are tolerated to reduce costs of herbivory in a community context (Kessler \& Baldwin, 2004).

In a community context, plants may play advanced strategies that match predictable patterns of herbivore attack. When arrival of one herbivore species predicts the odds of arrival of subsequent species, plants may evolve anticipation strategies (Mertens et al., 2021a,b). In such strategies the induced 
response to one herbivore includes a trait expression that prepares for future attack. Oviposition-induced resistance to caterpillar damage (Hilker \& Fatouros, 2015; Pashalidou et al., 2015), plants responding to snail cues and becoming more resistant to later arriving insect herbivores (Orrock et al., 2018), or leaf herbivory enhancing resistance to herbivores feeding on reproductive organs (McArt et al., 2013) are examples of this strategy. Anticipatory strategies may only persist under high predictability of herbivore co-occurrence and order of attack (Mertens et al., 2021b).

\section{Playing one or multiple strategies}

Adaptiveness of each of these strategies largely depends on community context and it is evident that many plant species are part of communities that are highly stochastic in terms of local dynamics of herbivore and pathogen attack. This not only means that within plant populations frequency-dependent selection may maintain large diversity in (plastic) defence strategies (Davila Olivas et al., 2017), but that individual plants may also be selected to play context-dependent strategies (Mertens et al., 2021b; Hervé et al., 2019). Within one plant the optimal defence strategy to play may depend on attack intensity. This may entail that depending on the severity of attack by a particular herbivore, plants may either integrate a response to multiple attackers at low density of each attacker or prioritize responses to one attacker when its density increases. Per plant organ, plants may play different strategies when leaves are under attack by a large diversity of herbivores, but reproductive tissues only by one specific attacker. The effectiveness of each strategy may also largely differ with plant ontogeny, especially when the costs of defence in trade-off with growth and the likelihood of attack vary across plant age (Barton et al., 2010; Ochoa-Lopez et al., 2020). This entails that individual plants may play ontogeny-determined plastic responses to multi-herbivore attack. Abiotic conditions may limit the potential to deploy particular strategies by resource limitation or by physiological interactions in dealing with abiotic and biotic stress (Nguyen et al., 2016). 


\section{BOX 2: Plant strategies}

In the context of the multitude of attackers arriving to plants in various ecological settings, a number of defence strategies can be defined. Many of these include advanced risk management strategies that are reflected in physiological responses of plants to herbivory.

Escaping: Plants respond to attackers with an induced shift in growth or phenology that reduces the encounter rate with the attackers (Van Wallendael et al., 2019).

Constitutive defence: Plants express their defences independently of actual herbivore attack (Erb, 2018; Karban, 2020).

General induced response: The induced response against the first attacker is also effective against all subsequent attackers (e.g. cell wall thickening, trichome density, broad spectrum of chemical defences) (Adio et al., 2011; Gonzales-Vigil et al., 2011)

Multifunctional defence traits: Plants use the same compound to defend against different attackers, or with different purposes (Li et al., 2018).

Canalization: The first herbivore attacking the plant has a large impact on the plant defence phenotype. The induced phenotype cannot be changed to respond against subsequent attackers and it determines how the plant interacts with other members of the insect community (Viswanathan et al., 2007).

Switching. The plant has the ability to always adapt the response to the current attacker and tailor the defence phenotype to the identity of the stressor.

Integration: Plants activate a defence response every time a new attacker arrives and integrate this response into a defence phenotype that is effective against the full suite of attackers, but sub-optimal to each individual attacker (Mathur et al. 2013; Stam et al., 2017).

Prioritization: Upon damage by the most ravaging herbivore species, plants target their defence to the specific attacker, with a cost of becoming more susceptible to other attackers.

Vaccination: Induced response to a herbivore species that can be tolerated with the benefit of reduced attack by a more ravaging herbivore (Kessler \& Baldwin, 2004).

Anticipation: Plants anticipate the arrival of other herbivores based on cues or presence of specific herbivores. In their response to the current attacker, the plant already mobilizes defences for likely forthcoming attack (Mertens et al., 2021a,b; McArt et al., 2013).

Priming: A physiological state of alert in response to environmental cues, that prepares the plant for future attack (Martinez-Medina et al., 2016). The alert state allows plants to rapidly respond to actual attack, not forming the defence in absence of the attacker. 


\section{CONCLUDING REMARKS AND FUTURE OUTLOOK}

Plant defence strategies against herbivores evolve under complexity of multi-herbivore attack, but have been largely studied in isolation over the past decades. Only very few studies have moved beyond challenging plants with two stressors (Kroes et al., 2016; Mathur et al. 2013; Stam et al., 2017), and these studies have only scratched the surface of how plants match their phenotype with natural challenges by a large diversity of herbivore attack (Fernández de Bobadilla et al., 2021). Important challenges are to identify plant limitations to deal with their natural number of stressors to also place our insights in defence plasticity to two attackers in context. In many of the plastic defence strategies we describe here, specific ecological settings may select against antagonistic interactions caused by induced plant responses. For example, when plants are consistently attacked by aphids and subsequently by caterpillars, we may anticipate that plants adapt their plasticity to this situation and reduce the impact of aphid-induced susceptibility to caterpillar attack by reducing antagonism between signalling pathways, correct those in downstream signal transduction or balance energetic costs of defence responses to multiple attackers. This may explain the absence of aphidinduced susceptibility to caterpillar attack in at least some plant species (Ali \& Agrawal, 2014; Mertens et al., 2021b; Moreira et al., 2018). Canalization or prioritization strategies may also identify the adaptive nature of antagonistic crosstalk in a community context rather than that this physiological concept may mismatch with the ecological outcome of plant-mediated species interactions.

Providing insights into the physiological strategies and limitations that plants have in dealing with multi-herbivore attack, we may start with uninformed and more artificial, controlled experiments. In these experiments, plants should be challenged with sequential herbivore attack beyond dual attack and address if plants prioritize responses to particular attackers or integrate all challenges into a compromised phenotype. Importantly, such studies should eventually be placed in an ecological context by challenging plants with natural 
sequences of herbivore attack potentially in comparison with non-occurring or rare orders of arrival to study what parts of plant plasticity are adaptive to patterns of attack found in nature (Mertens et al., 2021a). At least, we should conclude that in many of the plastic plant defence strategies that we outlined here, the signature of response to an individual stressor is likely to include an adaptation to the community of attackers as a whole. Thus, in transcriptomic studies on "the response of a plant to caterpillar or aphid attack", we are not just measuring the response to that particular species, but will observe regulation of processes that allow for resistance to other attackers (Mertens et al., 2021b). For example, physiological responses to the focal caterpillar we study include the preparation of the plant for likely pathogen attack in the open wound caused by the caterpillar or prepare the plant for arrival of other insect species that often co-occur with the caterpillar (Davila Olivas et al., 2019). The dependence of plant responses on the plant organ under attack, the density of the attacking herbivore and the time interval between herbivore arrival should be evaluated in the context of the strategies outlined here. This includes identification whether plants may connect local responses to leaf herbivory with anticipation of attack on reproductive organs such as flowers and ripening seeds.

A final challenge is to connect plant physiology and ecology to identify the ecological settings that correspond with the repertoire of defence plasticity of a plant. We should identify whether there is local adaptation by plant populations in defence plasticity to match their specific local pattern of attack, compare plant species in similar ecological context for their plastic defence strategies to multi-herbivore attack and compare related species to understand the macroevolutionary patterns of defence plasticity. 


\section{OUTSTANDING QUESTIONS}

To what extent does recognition of attack by specific herbivores lead to specificity in plant defence phenotypes?

What are plant limitations in physiological responses to multi-herbivore attack?

How is antagonistic cross-talk between signal transduction pathways matching multi-stress situations?

Is predictability in the order and timing of attack reflected in plastic plant defence strategies?

Are plants limited in deploying one strategy or can they play strategies depending on the ecological context?

ACKNOWLEDGEMENTS We thank Marcel Dicke for constructive comments on an earlier version of this manuscript. This work is supported by the European Research Council (ERC) under the European Union's Horizon 2020 research and innovation programme to E.H.P. (grant agreement 677139). 



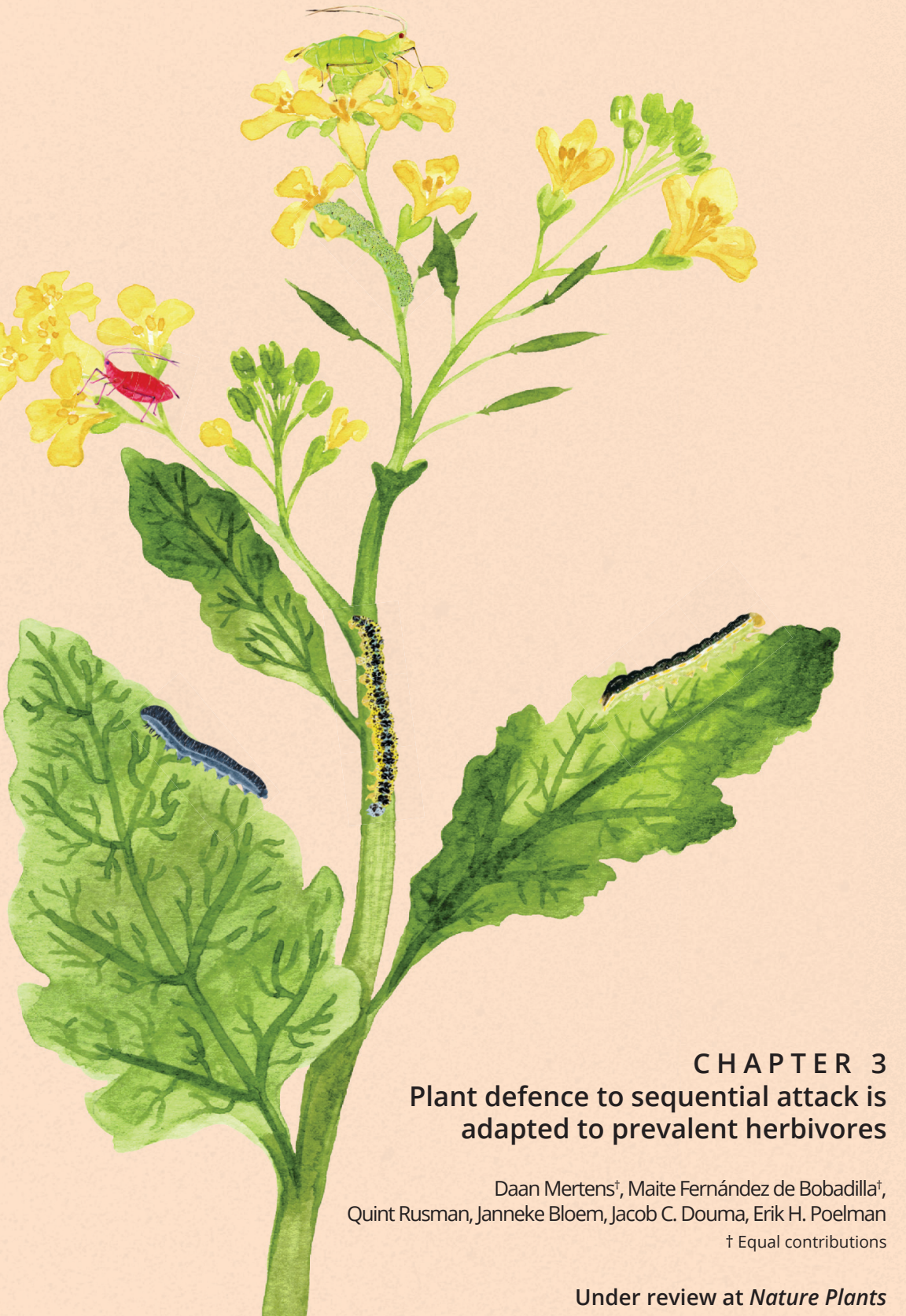





\section{ABSTRACT}

Plants have evolved plastic defence strategies to deal with uncertainty of when, by which species and in which order attack by herbivores will take place (Erb \& Reymond, 2019; Heil, 2010; Karban, 2020). However, the responses to current herbivore attack may come with a cost of compromising resistance to other, later arriving herbivores. Due to antagonistic cross-talk between physiological regulation of plant resistance to phloem-feeding and leaf-chewing herbivores (Ohgushi, 2005; Poelman et al., 2010; Erb et al., 2012; Soler et al., 2012; Thaler et al., 2012), the feeding guild of the initial herbivore is considered to be the primary factor determining whether resistance to subsequent attack is compromised. We show that, by investigating 90 pair-wise insect-herbivore interactions among ten different herbivore species, resistance of the annual plant Brassica nigra to a later arriving herbivore species is not explained by feeding guild of the initial attacker. Instead, the prevalence of herbivore species that arrive on induced plants based on three years of season-long insect community assessments in the field explained cross-resistance. Plants maintained resistance to prevalent herbivores in common patterns of herbivore arrival and compromises in resistance especially occurred for rare patterns of herbivore attack. We conclude that plants tailor induced defence strategies to deal with common patterns of sequential herbivore attack and anticipate arrival of the most prevalent herbivores. 
During 400 million years, insect herbivores have been driving the evolution of plant defences (Ehrlich \& Raven, 1964; Labandeira \& Johnson, 2002). Individual plants are typically attacked by multiple species of insects that arrive at different moments during a plant's lifetime. The occurrence of insect herbivores may be uncertain in terms of when, by which species, and in which order the attack will take place. To save costs of maintaining defences in the absence of herbivores, plants alter their level of defence in response to actual herbivore attack (Erb \& Reymond, 2019; Heil, 2010; Karban, 2020). These induced defences also allow plants to tailor resistance to the specific attacker which can be recognized by its damage pattern and compounds in the herbivore's oral secretion (Erb \& Reymond, 2019; Erb et al., 2012). However, the specific response to one herbivore may compromise the resistance to other herbivores. Physiological limitations in the regulation of resistance to sequential herbivore attack as well as manipulation of induced plant responses by herbivores may make plants more susceptible to secondary herbivore attack (Ohgushi, 2005; Poelman et al., 2010; Erb et al., 2012; Soler et al., 2012; Thaler et al., 2012).

Although plants are known to rapidly evolve to combinations of species they interact with (Ramos \& Schiestl, 2019; Züst et al., 2012), we know surprisingly little about the link between induced responses to herbivory and the odds of sequential herbivore attack that plants may have to deal with. The understanding of plant responses to attack by two sequentially attacking herbivore species is built upon a large collection of studies that arbitrarily selected pairs of herbivores to study plant resistance to sequential attack (Agrawal, 2000; Moreira et al., 2018) and in-depth studies of the underlying molecular mechanisms of plant physiological responses to dual attack (Erb \& Reymond, 2019; Voelckel \& Baldwin, 2004; Caarls et al., 2015; Proietti et al., 2018). These studies demonstrated that plant responses to attack by leaf-chewing herbivores such as caterpillars are primarily regulated through the jasmonic acid (JA) signalling pathway and generally result in induced resistance to subsequent attack by other leaf chewers (Davidson-Lowe et al., 2019; Eisenring et al., 2018). Plant responses to phloem-feeding herbivores such as aphids, primarily involve the salicylic acid 
(SA) signalling pathway and generally result in induced resistance to attack by other phloem-feeding herbivores (Züst \& Agrawal, 2016). However, a sequence of phloem-feeding and leaf-chewing herbivores compromises physiological responses to the later arriving species because of antagonistic crosstalk between the SA and JA pathways (Soler et al., 2012; Thaler et al., 2012; Caarls et al., 2015; Proietti et al., 2018; Ali et al., 2014). The current consensus is that plant responses to initial herbivore attack strongly determine the potential to respond to subsequent herbivory and results in compromises of resistance to sequential attack by herbivores of different feeding guilds (Thaler et al., 2012; Moreira et al., 2018). This consensus, based on physiological studies, has largely ignored the apparent negative ecological consequences of induced susceptibility to future herbivory (Poelman et al., 2010; Soler et al., 2012).

Empirical ecological studies and meta-analyses that compared interactions between several pairs of herbivores revealed high specificity in the magnitude of induced resistance or susceptibility in pairwise interactions (Agrawal, 2000; Moreira et al., 2018). In addition to feeding guild, diet breadth in terms of the level of food-plant specialization by herbivores is an important trait determining the nature of induced plant responses as well as the susceptibility of herbivores to the induced plant phenotype (Agrawal, 2000; Bidart-Bouzat \& Kliebestein, 2011). Whereas the feeding behaviour and growth of generalist herbivores is strongly affected by food-plant specific classes of chemical defences, specialist herbivores can cope with low concentrations of these compounds but are affected by high concentrations (Ali and Agrawal, 2014). Interestingly, closely related plant species differ in their responses and level of resistance to sequential attack by the same pair of herbivore species (Ali \& Agrawal, 2014). The apparent plant-species-specific responses to sequences of attackers indicate differences in selection pressures by sequential herbivory and suggest that plants are not systematically impaired in responding to sequential attack by herbivores of different feeding guilds. We argue that plant responses to initial herbivory should not result in significant compromises in resistance to subsequent attack by the most prevalent and damaging herbivore species. In optimizing their defence 
strategy, plants may prepare for likely future attack as part of their induced response to current attack (Mertens et al. 2021b).

Our study investigates whether the level of induced resistance to sequential herbivory in the annual plant Brassica nigra corresponds with the likelihood of the order and nature of sequential insect attack in the field. We link observations of the order of arrival of insect herbivores during three years of season-long insect community assessment on 488 plants, to a full-factorial assessment of induced resistance in pairwise plant-mediated interactions between ten herbivore species. We specifically test whether induced resistance of the second herbivore can be predicted by traits (feeding guild and diet breadth) of the inducing herbivore or the secondary receiving herbivore species, as well as the commonality of those species and their pairwise interactions in the field (Extended Data Figure 1, Supplementary Information, Table S1)

The ten herbivore species induced species-specific plant responses as characterized by the expression of LIPOXYGENASE 2 (LOX2) as marker gene for the JA pathway and PATHOGENESIS-RELATED PROTEIN 1 (PR1) as a marker for the SA pathway (Table S2) (Appel et al., 2014; Erb \& Reymond, 2019). As predicted from the literature (Bidart-Bouzat \& Kliebestein, 2011), chewing herbivores induced stronger JA responses compared to phloem feeders indicated by LOX2 expression levels but weaker SA responses indicated by PR1 expression levels (Extended Data Figure 2). These differences between feeding guilds were most apparent for specialist herbivores that also induced stronger LOX2 expression than generalist herbivores (Table S3-S6). However, the traits of the inducing herbivore (feeding guild and diet breadth) did not predict the performance of secondary herbivores feeding on the induced plants (Fig. 1, Table S7-8). Performance of receiving herbivores was either almost consistently promoted or consistently inhibited on plants previously induced by herbivores, independent of which herbivore species served as inducer (cf. rows in Fig. 1A). Surprisingly, we found much less consistency from the inducing herbivore perspective, as specific inducers could both promote or inhibit performance 


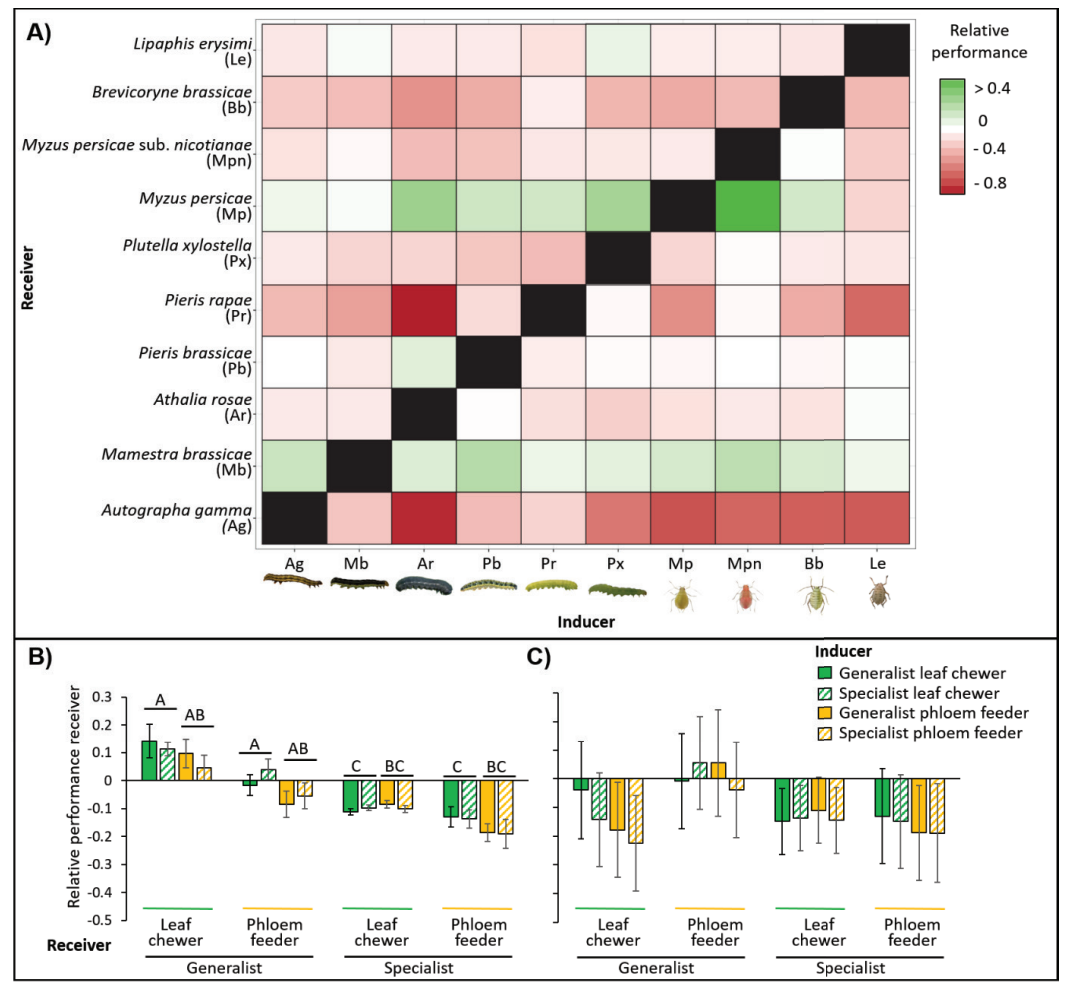

Figure 1. Effect of herbivory by ten primary herbivores (inducers) on the performance of the same ten species as secondary herbivores (receiver) relative to performance on an undamaged control plant. We modelled the performance (number of aphids, or larval weight) of each of the receiving herbivores as a function of the inducing herbivores. We calculated the response ratio for each observation by taking the natural logarithm of the ratio between the observed performance of an individual belonging to each species on a treatment and the modelled mean performance of all individuals of that species on control plants. The log response ratio (LnRR) is a dimensionless measure widely used in meta-analysis ${ }^{26}$. (A) Heat map as visual representation of the effect of each inducer on the relative mean performance of each receiving herbivore. (B) and (C) show relative performance of receiver based on the feeding guild and diet breadth of the inducer. Bars represent average relative performance \pm SE. Different letters indicate significant differences among treatments in post-hoc analyses (LMM). (B) Presents estimations from a model not correcting for inducer identity. (C) Corrected for inducer identity. 
of subsequent herbivores, even when those were of the same feeding guild (cf. columns in Fig. 1A). Thus, induction by leaf-chewing herbivores did not predict resistance to subsequent feeding by chewers, nor did herbivory by phloem-feeding herbivores predict resistance to subsequent phloem feeders. Moreover, induced responses to phloem feeders did not lead to susceptibility to secondary feeding by chewing herbivores. The plant phenotype induced by one herbivore species resulted in large variation in both sign and magnitude of effect on performance of the nine other secondary herbivore species (Fig. 1A, Extended Data Figure 3). These findings are further supported by the absence of a relation between the induced plant phenotype in terms of LOX2 and PR1 expression and secondary herbivore performance (Extended Data Figure 4). Gene expression of LOX2 and PR1 upstream in the defence signalling cascade may not predict expression of traits that affect herbivore performance such as primary metabolites, secondary metabolites (glucosinolates), or morphological defences such as trichomes (Supplementary Results and Discussion). Overall, secondary herbivore performance was best explained by diet breadth of the secondary herbivore, but was mostly independent of its feeding guild and largely driven by species-specific effects (Fig. 1B and 1C, Table S7-S10). Where most other studies have evaluated up to ten pairwise interactions, we show by using 90 species interactions (excluding intraspecific interactions) that the identity and/or functional group of the inducing herbivore does not affect the performance of a secondary herbivore on induced plants (Moreira et al., 2018). These findings ask for a shift of focus in our research field, stepping away from the predictive value currently attributed to the identity or functional group of inducing herbivores when inferring an ecological or evolutionary interpretation of induced plant responses to sequential herbivory. Instead, our data clearly show that the attack rates of individual herbivore species in the field are the major determinants of the level of cross-resistance in induced defence strategies.

Based on the assembly of insect communities over the lifetime of 488 plants, we found that five out of the 90 pairwise interactions investigated in our greenhouse experiment were not observed in the field. A total of 23 interactions 
occurred on less than $1 \%$ and 53 interactions were observed on less than $5 \%$ of the 488 plants, while 37 interactions were found on greater than $5 \%$ and 11 interactions were observed on more than $25 \%$ of the plants (Fig. 2D and Table S12-S14). The frequency of these observed species interactions is determined by overall prevalence of the inducing and secondary herbivore species, as well as by how initial herbivory affects the likelihood of a plant being colonized by a second herbivore species 5 . Our most important finding is that, when assessing the performance of herbivores on previously induced plants relative to their performance on untreated plants in our greenhouse experiment, we observed a reduced performance of specialist herbivores correlated with the increased prevalence of the herbivore under field conditions (Fig. 2A and 2B). This pattern is inversed when interactions involve a generalist secondary herbivore, showing that common interactions (occurring $>3.5 \%$ plants) are associated with higher performance of generalist secondary herbivores relative to their performance on non-treated plants (Fig. 2A and 2B). The frequency of interaction pairs to occur was strongly determined by the overall commonality of plant attack by specialist secondary herbivores (Table S11). Irrespective of the identity of the initial herbivore attacking the plant, resistance was strongest against prevalent secondary specialist herbivore species (Extended Data Figure 5). Even if plants were initially attacked by a prevalent phloem-feeding herbivore species, they were not compromised in resistance to prevalent specialist leaf-chewing herbivores. Thus, plant responses to initial herbivory did not compromise resistance to attack by common secondary herbivores (Extended Data Figure 5).

Placing our experimental findings in an ecologically relevant context is imperative for interpretation of the ecology and evolution of induced plant defences. First, the induced response to initial herbivory and enhanced resistance to specialist herbivores is likely adaptive as the insect community on B. nigra plants becomes dominated by the most ravaging specialist herbivores as the growing season progresses (Fig. 2C) (Connell, 1980). Moreover, herbivoreinduced Brassica plants are more frequently colonized by specialist herbivores than undamaged plants (Poelman et al., 2010). Specialist herbivores involved 


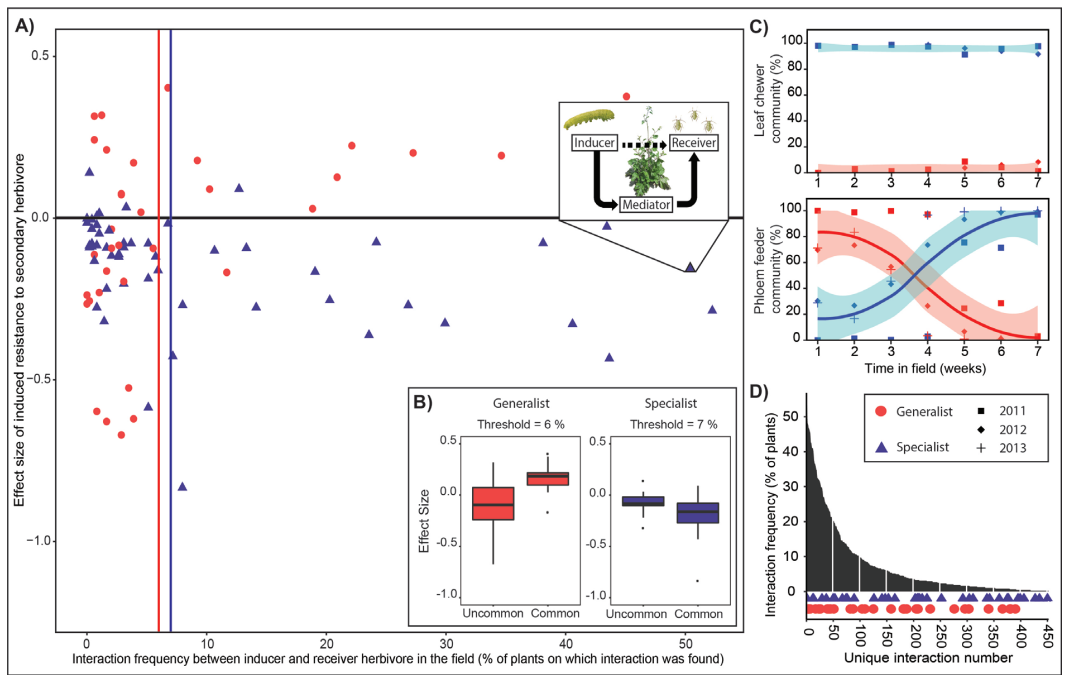

Figure 2. Optimal resistance strategies incorporate likelihood of secondary attack. (A) Outcome of pairwise interactions tested in a greenhouse set-up related to percentage of plants in our field experiment on which these interactions were observed. The coloured vertical lines represent the threshold set in B. (B) Response ratio of pairwise interactions classified as common or uncommon by an interaction frequency threshold. This threshold was set at $6 \%$ or $7 \%$ of plants in our field depending on whether the secondary herbivore was a generalist (left panel) or a specialist (right panel). These threshold values indicate the percentages of interaction occurrence that yielded clear significant differences between common and uncommon interactions in a sensitivity analysis for 20 threshold values. Response ratio of common and uncommon interactions were significantly different (Mann-Whitney test; generalist $p=0.004$ and specialist $p=$ 0.002). (C) Ratio of specialist and generalist herbivores in the field experiments over time, indicating increasingly specialist dominated herbivore communities. (D) The percentage of plants on which the 453 unique interactions observed in the field occurred. Circles and triangles indicate the interactions we tested in our greenhouse set-up.

in the most common interactions were also the most prevalent herbivores and colonize individual plants with larger numbers than herbivores in rare interactions (Table S13-S14). Together these findings indicate that B. nigra plants activate defence strategies to the most likely type of herbivore attack and are not compromised to deal with these attackers by responses to initial herbivory. However, compromises do arise when later arriving herbivores are 
rare. Second, we observe apparent maladaptation of induced plant responses to secondary herbivores with a wide diet breadth (generalists) (Fig. 2A and 2B). We propose that the costs of this induced susceptibility are relatively small due to the effectiveness of constitutive resistance (Barton et al., 2010). Even though relative performance of surviving generalist herbivores on induced plants compared to undamaged plants was positive, the mortality rates were high compared to specialist herbivores, and the size of those that survived were small (Extended Data Figure 3). Third, strong negative effects on rare secondary herbivores can be explained by non-mutually exclusive hypotheses (Fig. 2A). They may indicate an evolutionary signature of past arms races between B. nigra and the specific herbivore species (Connell, 1980). Alternatively, the strong, induced cross-resistance may indicate resistance to a secondary herbivore that has strong outbreak cycles that did not occur during the three-year period of our field observations (Barbosa et al., 2012). Finally, some of the resistance to uncommon secondary herbivores may be due to trait overlap with secondary herbivore species involved in common interactions and hence similarity in performance on induced plants.

The results of this study demonstrate that B. nigra plants optimize their defence responses to herbivory by anticipating the arrival of the most abundant herbivores in response to initial herbivory. To understand the evolution of induced defence strategies, plant physiological responses to single herbivore attack and its plasticity to multi-herbivore attack should thus be evaluated in a community perspective, including the dynamic patterns in order and timing of multi-herbivore attack. The adaptive significance of plant responses to initial attack is largely determined by the predictability of secondary herbivore attack and herbivore traits such as diet breadth. The cross-talk between SA and JA should not be extrapolated to ecological outcomes of herbivore interactions, because substantial variation in plant defence phenotypes may arise after initial expression of genes basal to the JA and SA signal transduction pathways (Züst et al., 2012). SA and JA cross-talk in plants may dampen the overly strong induction of specific defensive phenotypes and allow a fine-tuning where the 
urgent need to respond to current stress is traded off to retain the capability to respond to probable subsequent stresses (Thaler et al., 2012). We speculate that initial attack by any herbivore species may result in plants mobilizing resistance to tailor future prevalent herbivores. Thus, the simultaneous upregulation of JA and SA related genes to initial attack found in many studies on induced responses to individual herbivores could presumably be responses targeted to predictable future attack (Mertens et al., 2021b). For instance, PR1 expression in response to chewers may predict that plants are exposed to a large likelihood of future aphid attack. Hence, plants optimize their defence strategy to match common patterns of sequential herbivore attack, to not compromise responses to the most prevalent herbivore attacks.

\section{METHODS}

\section{Study system}

Brassica nigra (L.) Koch is an annual herbaceous plant common throughout Europe and used as host plant by a wide range of phytophagous insects (Bischoff \& Trémulot, 2011; Schlinkert et al., 2015). Seeds collected from a local population in Wageningen, The Netherlands, were germinated in trays. One-week-old plants were transplanted and grown in pots (ø $15 \mathrm{~cm}, 1 \mathrm{~L})$ under greenhouse conditions $\left(22 \pm 2{ }^{\circ} \mathrm{C}, 60-70 \%\right.$ r.h. and L16:D8). Four-week-old plants were used in greenhouse experiments, seedlings were planted in field experiments.

Ten insect species were used as herbivores (Table S1). We used first instar larvae of the leaf chewers: the silver Y, Autographa gamma (Ag) (Lepidoptera: Noctuidae); the cabbage moth, Mamestra brassicae (Mb) (Lepidoptera: Noctuidae); the large cabbage white, Pieris brassicae (Pb) (Lepidoptera: Pieridae); the small cabbage white, P. rapae (Pr) (Lepidoptera: Pieridae); the turnip sawfly, Athalia rosae (Ar) (Hymenoptera: Tenthredinidae); and second instar larvae of the diamondback moth, Plutella xylostella $(\mathrm{Px})$ (Lepidoptera: Plutellidae). Starting mass of these newly hatched herbivores is below the error margins of an analytical balance and was not assessed. We used adult wingless individuals of 
the phloem feeders: the cabbage aphid, Brevicoryne brassicae $(\mathrm{Bb})$; the green peach aphid, Myzus persicae (Mp); the tobacco aphid, My₹us persicae sub. nicotianae (Mpn); and the mustard aphid, Lipaphis erysimi (Le) (all Hemiptera: Aphididae). The insects A. gamma, M. brassicae, P. brassicae, P. rapae, B. brassicae and P. xylostella were reared on Brussels sprouts (Brassica oleracea L. var. gemmifera cv. Cyrus), while $A$. rosae, $M$. persicae, $M$. persicae sub. nicotianae and $L$. erysimi, were reared on radish (Raphanus sativus) (all from the stock rearing of the Laboratory of Entomology, Wageningen University). The insect cultures were kept under greenhouse conditions $\left(22 \pm 2{ }^{\circ} \mathrm{C}, 50-70 \%\right.$ r.h. and L16:D8). We classified herbivore species as host specialists when their documented trophic niche in the relevant herbivorous life stages is limited to plants of Brassicaceae (Table S1). The herbivore species were selected for being culturable, the possibility to measure a proxy for their performance (weight gain or population growth) and to represent a balanced spectrum of feeding guilds, level of food plant specialisation and prevalence in the field.

\section{Experimental design}

To assess plant defence responses to herbivory and the ecological outcome of all 90 pairwise interactions among the ten different insect herbivore species in our experiment, we divided the experiment in ten blocks. In each experimental block, we used all herbivore species as inducers and only one of the ten species as secondary herbivore (receiver) (Extended Data Figure 1). Each block consisted of 110 plants, with ten plants assigned to each of the herbivore inducer treatments, and ten plants which were left untreated. Treatments were randomly arranged over two benches in a greenhouse compartment $(22 \pm 2$ ${ }^{\circ} \mathrm{C}, 60-70 \%$ r.h. and L16:D8). To prevent desiccation of the plants and cross contamination between treatments, we placed plants in inundated trays. We infested the youngest fully developed leaf of each plant with either ten wingless aphids or five first instar larvae. All herbivores were left free to move on the plant, allowing them to choose their preferred feeding sites. 


\section{Plant transcriptional responses to herbivore induction}

As a measure for plant defensive responses to each of the ten initial herbivores, we quantified levels of transcription of two marker genes in Brassica nigra; LIPOXYGENASE 2 (LOX2) for the jasmonic acid signalling pathway (JA) (Snoeren et al., 2011) and PATHOGENESIS-RELATED PROTEIN 1 (PR1) for the salicylic acid signalling pathway (SA) (Leon-Reyes et al., 2010) (Table S2). In each experimental block, leaf samples were taken at 24 or 96 hours after plants were infested with herbivores. The time points for LOX2 and PR1 expression were selected based on their peaks of expression after herbivory in Brassica nigra identified in previous studies (Broekgaarden et al., 2011; Fernández de Bobadilla et al., 2021). For each herbivore treatment and time point, we sampled the youngest fully developed leaf on which herbivores had been released from five of the plants. These leaves were always damaged and had the majority of released herbivores still present. Samples were taken by detaching the leaf with a razorblade and punching a leaf disc of $2 \mathrm{~cm} \varnothing$ with a leaf puncher. Herbivores present on the detached leaf were placed back on the plant. The five leaf discs were combined to constitute one biological replicate, submerged in liquid nitrogen right after sampling, and stored at $-80{ }^{\circ} \mathrm{C}$ until further analysis. Per experimental block, a single biological replicate per inducer and time point was obtained, yielding a total of ten biological replicates per treatment and time point for the entire experiment. The frozen samples were ground to a fine powder with a pestle, and RNA was isolated from the plant material by using RNeasy Plant Mini Kit (Qiagen) and treated with DNAse 1 (Invitrogen) following the manufacturer's instructions. After isolation, the RNA concentration was quantified using a NanoDrop ND-100 spectrophotometer (NanoDrop Technologies, Wilmington, DE, USA; all samples with OD 260 $\mathrm{nm} / 280 \mathrm{~nm}$ of 1.9-2.2 ratio). Samples were diluted and adjusted to an RNA concentration of $50 \mathrm{ng} / \mu \mathrm{l}$. From the RNA samples we synthesized cDNA, using the SensiFAST cDNA synthesis kit (Bioline). We quantified expression levels of each sample by RT-qPCR, using the SensiFAST SYBR no-ROX kit (Bioline). Five ng of the cDNA template were added to the reaction with a total volume of $20 \mu \mathrm{L}$. The reactions were performed with shuttle PCR conditions using 
a CFX96 Touch ${ }^{\mathrm{TM}}$ Real-Time PCR Detection System (Biorad). All reactions were conducted using two technical replicates, and samples were omitted from further analysis if the difference in expression between the technical replicates was higher than 0.5. Plate setups also included negative controls (no template) and inter-run calibrators. Gene-expression data was imported to qBase +3.1 (Biogazelle), to calculate Calibrated Normalized Relative Quantity (CNRQ). The CNRQ value represents the relative quantity (gene expression level) of a sample for a given target gene corrected by the expression value of two reference genes for each sample. We tested the following reference genes for expression stability: ACTIN-2 (ACT2), BETA-TUBULINE (B-TUB), ELONGATION FACTOR-1 (EF1), PEROXIDASE 4 (PER4), SECRETION ASSOCLATED RAS RELATED GTPASE 1 A (SAR1A), GLYCERALDEHYDE-3-PHOSPHATE DEHYDROGENASE (GAPDH). The last two genes were selected as reference genes because they had highest stability (i.e. their expression was not affected by the treatment). Data was corrected for differences between runs using inter-run calibrators.

\section{Herbivore performance}

To quantify pairwise plant-mediated interactions among the full matrix of interactions between the ten herbivores (90 interactions, excluding intraspecific interactions), we assessed herbivore performance on herbivore-induced plants. These were the same plants that were sampled for gene expression analyses, allowing for direct correlations between defence gene expression and herbivore performance. Seven days after initial herbivore (inducers) infestation, we removed all herbivores (to exclude direct effects of inducing herbivores on the receiver herbivore and thus isolate plant-mediated effects through herbivoreinduced plant responses) and immediately re-infested plants with the second herbivore species (receivers). When removing herbivores, we retrieved inducing herbivores from all plants which were clearly damaged by these herbivores. We placed either 20 wingless aphids or ten first instar larvae of the secondary herbivore (receiving) species on all plants in a block, repeating the setup with a different herbivore receiver in each block. After seven days we assessed the 
performance of the receiving herbivore by either counting the number of aphids or by collecting and weighing larvae individually (scales: Sartorius CP2P, Mettler Toledo ML54/01) (Extended Data Figure 1). Aphids were counted twice by different persons, and the average was taken as measure of their performance. Herbivore performance is commonly used as parameter of plant resistance to herbivory and provides insight in how initial herbivory results in induced resistance or susceptibility to secondary herbivores.

\section{Herbivore interaction frequency under field conditions}

Information on herbivore interaction frequency under field conditions was collected during three years of common garden experiments (summers of 2011, 2012 and 2013) in an experimental field in Wageningen, The Netherlands. We recorded herbivore identity and time of arrival on a total of 540 B. nigra plants (180 plants per year). Insect presence was assessed weekly, surveying herbivore community development on individual plants from seedling until seed maturation. Plants that were monitored less than 4 times were removed from the dataset, retaining 488 plants and 895,236 herbivore observations in the analysis (Table S14). We calculated the relative number of plants on which we recorded one herbivore species arriving before or at the same time as another herbivore species. The 90 pairwise interactions tested in our greenhouse experiments spanned the range from common to rare among all pairwise interactions identified on B. nigra plants in the field (Fig. 2D).

\section{Statistical analysis}

For each modelling analysis, we selected the best model from a set of candidate models by comparison of the Akaike information criteria (AIC) with a selection threshold of $\Delta 5$ AIC (Johnson et al., 2004). Candidate models differed in the number of fixed factors (and their interactions) and the stochastic distribution. Full models included all interactions between fixed factors and in case mixed models were applied they included all random intercepts. When mixed effect models were applied, we first optimized the random structure, and then the fixed structure of the model (Zuur et al., 2009). When more than 
one model was optimal based on our AIC threshold, we explored all optimal models to verify that our interpretation of the results would not differ among models and reported the model with lowest AIC. We further compared the AIC of optimal models with the AIC of null models which did not contain any predictors (i.e. the model assumes the effect of all fixed factors is equal to zero). Parameters of the optimal model were estimated by restricted maximum likelihood estimation or Laplace approximation. After model selection, we evaluated the significance of different factors in the optimal (generalized) linear (mixed) model (see Plant transcriptional responses to herbivore induction and Herbivore performance). Pairwise post-hoc comparisons were evaluated by Tukey's HSD test and contrasts were considered significantly different at $P$-values $\leq 0.05$. Statistical analyses were done using the nlme (Pinheiro et al., 2019), lme4 (Bates et al., 2015), Imtest (Zeileis \& Hothorn, 2002), and emmeans (Lenth, 2018) packages in $\mathrm{R}$ (v3.2.4, R Core Team 2016).

\section{Plant transcriptional responses to herbivore induction}

To model species-specific effects of herbivore induction on the relative expression of LOX2 and PR1 we applied generalized linear models (GLM) with gamma error distribution and log link function. The full models included herbivore species identity and the time point at which the sample was taken $(24 \mathrm{~h}$ or $96 \mathrm{~h}$ after induction) as explanatory factors. To detect broader patterns based on the feeding behaviour of the herbivores, we performed a second analysis. Here we applied generalized linear mixed models (GLMM) with gamma error distribution and inverse link function to evaluate effects of the inducing herbivore's feeding guild (phloem feeder or chewing herbivore), its level of host specialization (specialist or generalist), and the time point at which the sample was taken (24h or 96h after induction) on the relative expression of LOX2 and PR1. To interpret patterns in transcription levels in terms of feeding guild and host specialization of herbivores while correcting for variable species effects within feeding guild and host specialization, species identity was taken as a random effect. As we were interested in species-specific effects on relative gene expression and exploring patterns on higher functional levels (i.e. herbivore 
feeding guild and host specialization) rather than changes in expression over time, we performed pairwise comparisons for the two time points separately.

\section{Herbivore performance}

To allow comparison of performance across all herbivore species, we calculated the response ratio (LnRR) for each observation (number of aphids, or larval weight) by taking the natural logarithm of the ratio between the observed performance $(\mathrm{X})$ of an individual (i) belonging to species (S) on treated plants $(\mathrm{T})$, and the mean performance of species $(\mathrm{S})$ on control plants $(\mathrm{C})$ :

$$
\begin{aligned}
& \mathrm{LnRR}=\begin{array}{c}
\text { individual performance on induced plant } / \\
\text { average species performance on control plants }
\end{array} \\
& \mathrm{LnRR}=\ln \left[\mathrm{X}_{\mathrm{iST}} /\left(\mathrm{X}_{\mathrm{SC}}\right)\right]
\end{aligned}
$$

The response ratio is a practical measure when comparing strength and sign of treatment effects across multiple experiments and different response variables, and is widely used in ecological meta-analysis (Hedges et al., 1999; Lajeunesse, 2011). To get robust estimates of mean performance on control plants, we fitted mixed effect models expressing the herbivore performance (number of aphids or larval weight) as a function of the induction treatment, and including the plant individual and the timepoint at which the leaf sample was taken as random factors. The estimated means on control plants were used as denominator in the response ratios. We applied linear mixed effect models (LMM) for each of the phloem-feeding herbivores and Pieris brassicae, and generalized linear mixed models (GLMM) with gamma error distribution and $\log$ link function for each of the remaining leaf chewing herbivores. We found heterogeneity of variance in the models estimating phloem feeder performance and in the model estimating $P$. brassicae performance. To adjust for this, we allowed variance to be different for each of the inducing herbivores.

Next, we evaluated if herbivore performance depended on the inducing or receiving herbivore. We applied a linear mixed effect model (LMM) with 
response ratio as dependent variable and inducing and receiving herbivore identity as independent variables. We corrected for dependency between observations by including the plant individual as random intercept and adjusted for heterogeneity of variance by allowing variance to be different for each of the receiving species. The alternative model that was tested was a model that related the response ratio to the feeding guild (phloem feeder or chewing herbivore) and level of host specialization (specialist or generalist) of the inducer and receiver herbivores with plant individual as random effect. We excluded pairwise interactions between the herbivores themselves, and between Myzus persicae sub. nicotianae and $M$. persicae from this analysis, as for the latter the data obtained was an outlier in effect size. Although including this interaction did not significantly change the interpretation of our results, it was influential in determining confidence intervals and AIC values, hampering convergence of analysis of the full matrix of interactions. In a third analysis we added a random effect including species identity of both the inducing and receiving herbivore to the model evaluating feeding guild and level of host plant specialization.

In a final analysis we assessed the role of prevalence of both the inducing and receiving herbivores as observed under field conditions in predicting the performance of the receiving herbivore. In these models, the estimated response ratios of pairwise interactions was included as dependent variable, and the feeding guild (phloem feeder or chewing herbivore), level of host specialization (specialist or generalist) and prevalence of the inducer and receiver herbivores were included as explanatory variables. The backward selection procedure applied to optimize the model converged on an optimum that included a complex four-way interaction. Hence, to avoid overinterpretation of our data, we chose to optimize the model by applying a forward stepwise selection procedure.

\section{Relation to field observations}

To evaluate whether patterns in herbivore performance under greenhouse conditions could be linked to interaction frequency under field conditions, we related the frequency of each tested pairwise interaction observed in the field 
to their estimated mean response ratio in the performance assays. We annotated interactions based on host specialization of the receiving herbivore (host specialists or generalists), and analysed the two groups separately. We classified interactions as either common or uncommon for a range of 20 threshold values, based on the percentage of plants on which interactions were observed (i.e. interaction frequency). To statistically compare the mean response ratio of common and uncommon interactions at each of the 20 threshold values, we applied a non-parametric rank-based test (Mann-Whitney). To assess variability of the observed effect size independent of the sign of the effect, we transformed the estimated mean response ratio by taking its absolute value. This transformed dataset was subjected to the same non-parametric analysis with 20 threshold values of interaction frequencies used in our previous analysis. As the number of common or uncommon interactions was dependent on the assessed interaction frequency threshold, we compared the variance of the group with the least number of pairwise interactions with the variance of an equal number of randomly selected interactions from the largest group. This permutation procedure was repeated 1,000 times for each assessed threshold level. We used prevalence of herbivores (the number of plants colonized by a specific species) as a measure for the importance of interactions, instead of the abundance of the herbivore in the field (number of individuals counted over all plants). Abundance of herbivores on an individual plant may not accurately compare fitness costs of herbivory across species, since the costs of attack by a large number of aphids may not compare to attack by a single ravaging caterpillar.

ACKNOWLEDGMENTS We thank Anurag Agrawal and Marcel Dicke for discussion of our results; We acknowledge funding by the European Research Council (ERC) under the European Union`s Horizon 2020 research and innovation program (grant agreement nr: 677139 to EHP). JCD was supported by a NWO Earth and Life Sciences (NWO-ALW) through a VENI grant, project no. 863.14.018; 
AUTHOR CONTRIBUTIONS EHP, DM and MFB planned and designed the study and developed the methodology; EHP, DM, MFB, QR and JB performed the experiments; MFB and JB analysed gene expression; DM and BD analysed performance and field data; all authors contributed to writing of the manuscript;

COMPETING INTERESTS Authors declare no competing interests;

DATA AND MATERIALS AVAILABILITY All data is available in the main text or the supplementary materials.

ADDITIONAL INFORMATION Supplementary Information is available for this paper. 


\section{EXTENDED DATA FIGURES}

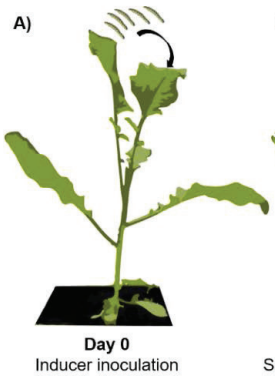

Inducer inoculation
B)

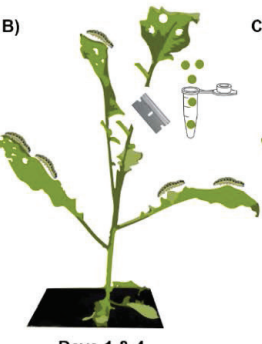

Days 1 \& 4

Sampling for gene expression

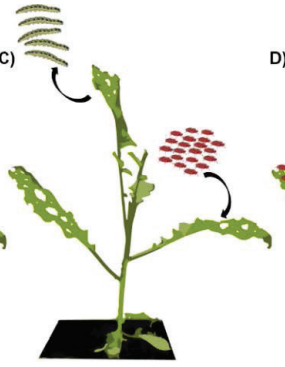

Day 7

Remove inducers Remove inducers
Inoculate receivers

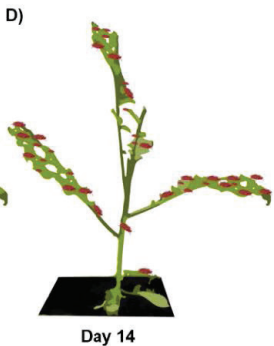

Measure performance receiver

Extended Data Figure 1. Experimental setup for the performance experiment. A) Brassica nigra plants were infested with one of ten insect species as primary herbivores (inducers). Five leaf chewers or ten phloem feeders were used as inducers. B) One and four days after plant infestation with herbivores, leaf samples for gene expression analyses were taken (each plant was sampled only once). C) Seven days after plant infestation with herbivores, all remaining inducers were removed and plants were infested with secondary herbivores (receivers). Ten leaf chewers or 20 phloem feeders were used as receivers. D) Seven days after plant infestation with receivers, their performance was measured (leaf chewer weight or number of phloem feeders). We repeated this setup ten times, each time using a different insect as receiver, and preparing ten plant replicates per treatment (Table S1). 

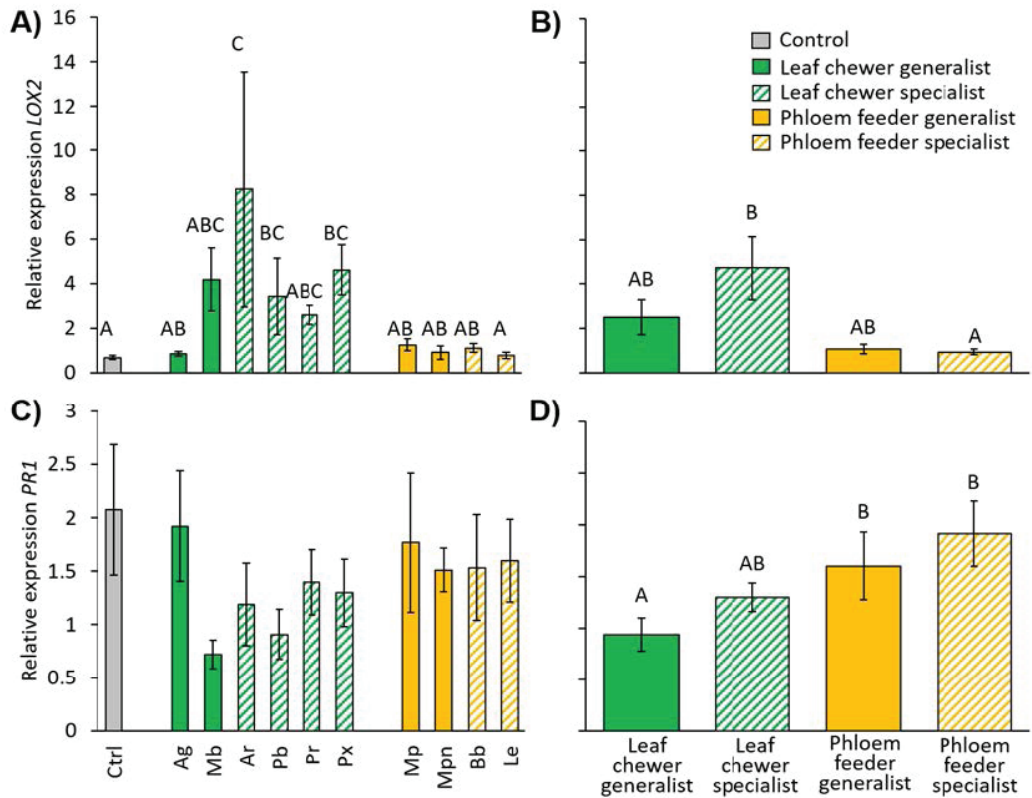

Extended Data Figure 2. Expression levels of the JA biosynthesis gene LOX2 and the SA responsive gene PR1 on Brassica nigra leaves after 96h of herbivory. Herbivores used were the leaf chewers Autographa gamma (Ag), Mamestra brassicae (Mb), Athalia rosae (Ar), Pieris brassicae $(\mathrm{Pb})$, P. rapae $(\mathrm{Pr})$ and Plutella xylostella $(\mathrm{Px})$, and the phloem feeders Myzus persicae (Mp), M. persicae sub. nicotianae (Mpn), Brevicoryne brassicae (Bb) and Lipaphis erysimi (Le). A) Effects of herbivory on the relative expression of LOX2 for each herbivore species. B) Effects of herbivory on the relative expression of LOX2 for herbivores grouped by feeding guild and diet breadth. C) Effects of herbivory on the relative expression of PR1 for each herbivore species. D) Effects of herbivory on the relative expression of $P R 1$ for each herbivore species grouped by feeding guild and diet breadth. Bars represent mean \pm SE. Different letters refer to significant differences at $P<0.05$ based on GLM and Tukey HSD tests (Table S3). 


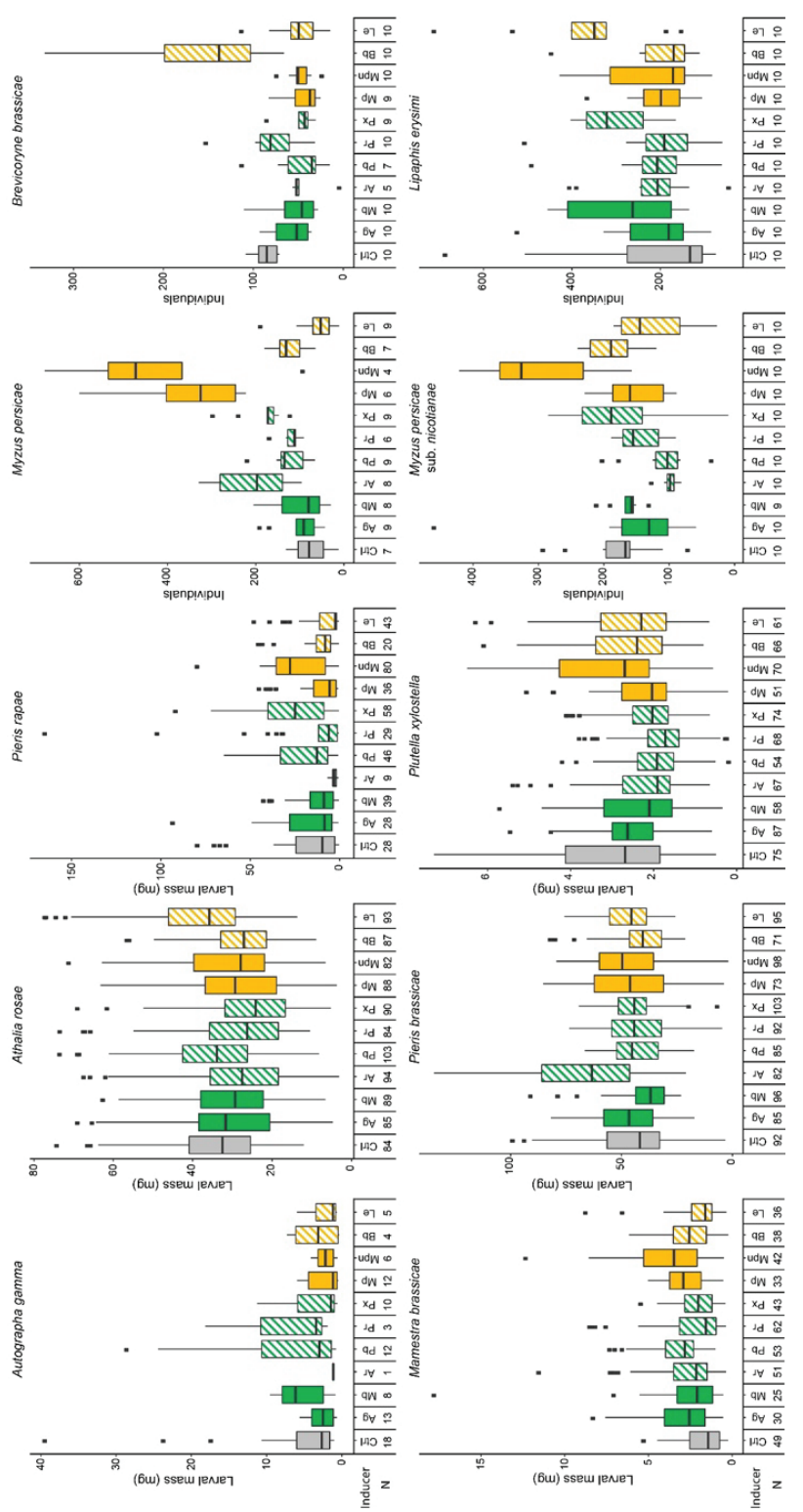

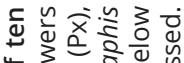
t

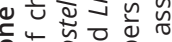

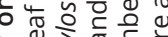

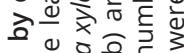

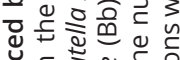
긍 웡 원

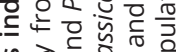

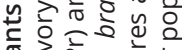

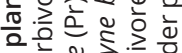

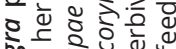

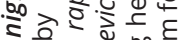

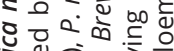
ทั่ 후응요웡

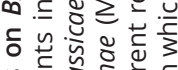

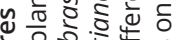
으는원

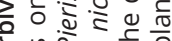

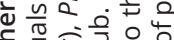

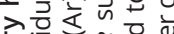

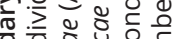
으든 는

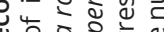
ปั ט

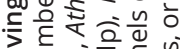

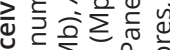
능 ¿ ᄂ

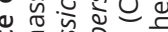

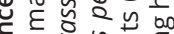

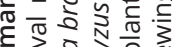

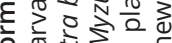
은 竎 $\sum_{0}$ ขं ह $\frac{1}{0}$

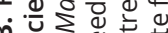
m บ $\sum \Phi$ 는 은 อ

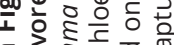

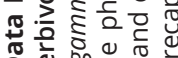

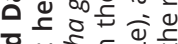

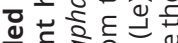

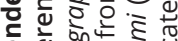

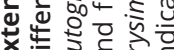




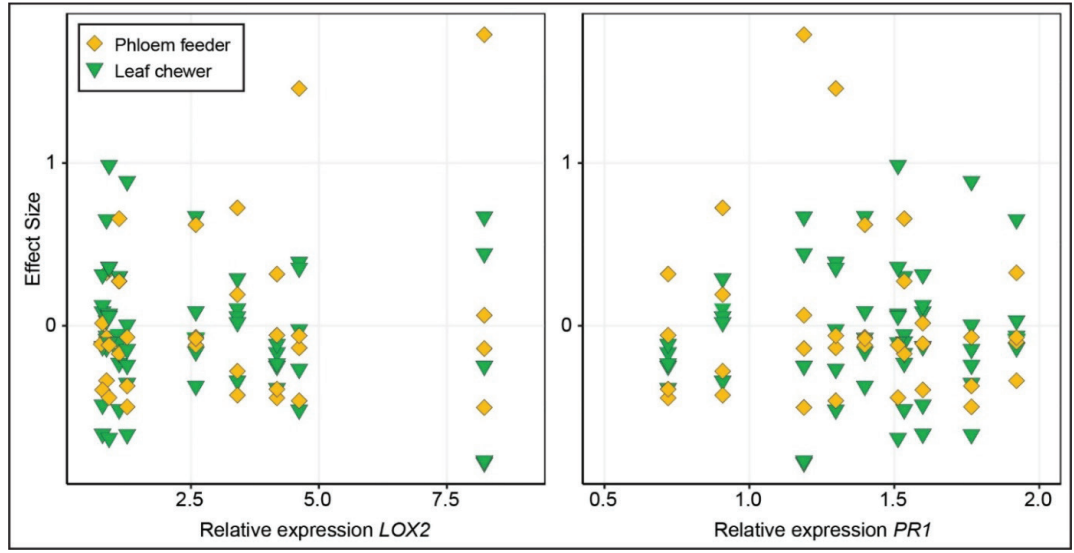

Extended Data Figure 4. Performance of herbivores is not explained by marker gene expression. Performance of receiving herbivores (effect size; the natural logarithm of the ratio between individual performance on induced plant / average species performance on control plants) related to the relative expression of the JA-marker gene LOX2 (left panel) or the SA-marker gene PR1 (right panel). Colours and shapes correspond to the feeding guild of the receiving herbivore. 

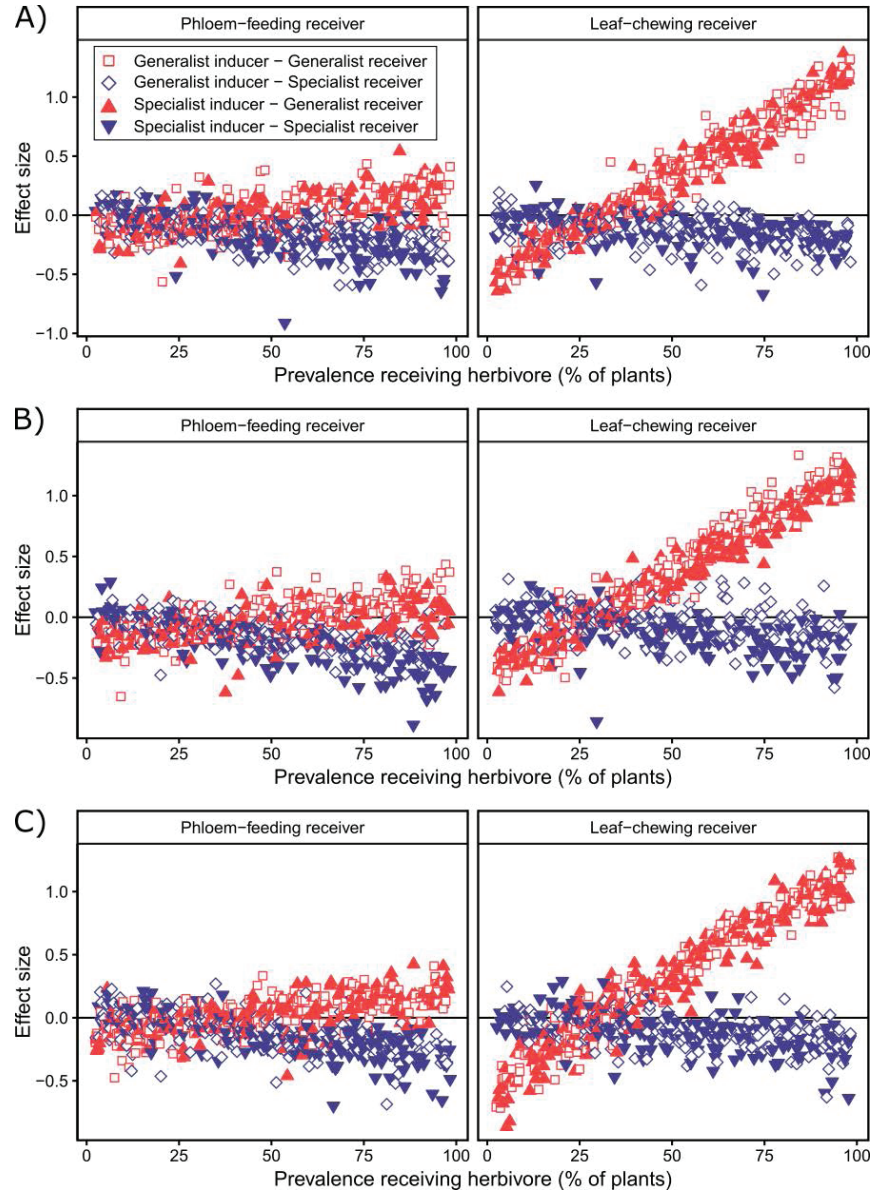

Extended Data Figure 5. Resistance strategies are predicted by prevalence of the receiving herbivore. Model predictions of performance of receiving herbivores (effect size; the natural logarithm of the ratio between individual performance on induced plant / average species performance on control plants) related to the prevalence of the receiving herbivore, separated for phloem-feeding herbivores (left panels) and leafchewing herbivores (right panels). Colours and shapes correspond to combinations of diet breadth of the inducing and receiving herbivores. A) Model predictions did not constrain the prevalence of the inducing herbivore, B) predictions were constrained in prevalence of the inducing herbivore at $2.5 \%$ of plants. C) predictions were constrained in prevalence of the inducing herbivore at $50 \%$ of plants. 
Table S1. Insects used in the greenhouse experiments, their diet breadth and feeding guild. In brackets abbreviations used for species identification in the text and figures.

\begin{tabular}{|c|c|c|c|}
\hline & Species (Abbreviation) & Diet breath & Feeding guild \\
\hline & Autographa gamma (AG) & Generalist & Leaf chewer \\
\hline & Mamestra brassicae (Mb) & Generalist & Leaf chewer \\
\hline & Athalia rosae (Ar) & Specialist & Leaf chewer \\
\hline 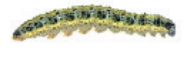 & Pieris brassicae $(\mathrm{Pb})$ & Specialist & Leaf chewer \\
\hline wine & Pieris rapae (Pr) & Specialist & Leaf chewer \\
\hline m & Plutella xylostella $(\mathrm{Px})$ & Specialist & Leaf chewer \\
\hline & Myzus persicae (Mp) & Generalist & Phloem feeder \\
\hline & Myzus persicae sub. nicotianae (Mpn) & Generalist & Phloem feeder \\
\hline & Brevicoryne brassicae $(\mathrm{Bb})$ & Specialist & Phloem feeder \\
\hline & Lipaphis erysimi (Le) & Specialist & Phloem feeder \\
\hline
\end{tabular}

Table S2. Four genes used in qRT-PCR to quantify defensive responses of Brassica nigra to herbivory.

\begin{tabular}{|c|c|c|c|c|}
\hline Target & Full name & Pathway & $\begin{array}{l}\text { Forward sequence } \\
\qquad\left(5^{\prime}-3^{\prime}\right)\end{array}$ & $\begin{array}{c}\text { Reverse sequence } \\
\left(5^{\prime}-3^{\prime}\right)\end{array}$ \\
\hline$\angle O X 2$ & LIPOXYGENASE & JA & $\begin{array}{l}\text { TGCTCGTGCACGC- } \\
\text { CAGAGTC }\end{array}$ & $\begin{array}{l}\text { AGCCAGCCCCCT- } \\
\text { GCTGATGA }\end{array}$ \\
\hline$P R-1$ & $\begin{array}{l}\text { PATHOGENESIS- } \\
\text { RELATED PROTEIN } 1\end{array}$ & SA & $\begin{array}{l}\text { CGCCGACGGAC- } \\
\text { TAAGAGGCG }\end{array}$ & $\begin{array}{l}\text { ACACCTCGCTTTGC- } \\
\text { CACATCCA }\end{array}$ \\
\hline SAR1A & $\begin{array}{c}\text { SECRETION } \\
\text { ASSOCIATED RAS } \\
\text { RELATED GTPASE } 1 \mathrm{~A}\end{array}$ & $\begin{array}{l}\text { Reference } \\
\text { gene }\end{array}$ & $\begin{array}{c}\text { ATCTCTAGCCACCG- } \\
\text { TTCCCT }\end{array}$ & $\begin{array}{c}\text { TTCCTGACGATGCT- } \\
\text { GCACAT }\end{array}$ \\
\hline GAPDH & $\begin{array}{l}\text { GLYCERALDEHYDE- } \\
\text { 3-PHOSPHATE } \\
\text { DEHYDROGENASE }\end{array}$ & $\begin{array}{l}\text { Reference } \\
\text { gene }\end{array}$ & $\begin{array}{l}\text { GGAGCTGCCAAG- } \\
\text { GCTGTCGG }\end{array}$ & $\begin{array}{c}\text { CCTTCAGA- } \\
\text { TTCCTCCTTGATA- } \\
\text { GCC }\end{array}$ \\
\hline
\end{tabular}


Table S3. Effects of the inducing herbivore's identity and time of sampling ( $24 \mathrm{~h}$ or $96 \mathrm{~h}$ ) on relative expression of LOX2 of Brassica nigra plants. A. Main model selection steps indicating AIC values of the full model (including all factors and their interaction), the null model (assuming all factors equal to zero), and the optimal model (lowest AIC value). B. Results from the optimal model (generalized linear model (GLM) with gamma error distribution and log link function). Values in bold denote significant factors $(P<0.05)$.

\begin{tabular}{lll}
\hline A. & \\
\hline Model & Expression & AIC \\
\hline Full & $L O X 2 \sim$ Inducing Herbivore * Time & 633 \\
\hline Optimal & $L O X 2 \sim$ Inducing Herbivore + Time & 626 \\
\hline Null & $L O X 2 \sim 1$ & 761
\end{tabular}

\begin{tabular}{llll}
\hline B. & & & \\
\hline Fixed factor & $\boldsymbol{\chi}^{\mathbf{2}}$ & df & $\boldsymbol{P}$ \\
\hline Inducing Herbivore & 140.18 & 10 & $<\mathbf{0 . 0 0 1}$ \\
\hline Time & 11.602 & 1 & $<\mathbf{0 . 0 0 1}$ \\
\hline
\end{tabular}

Table S4. Effects of herbivore feeding guild (phloem feeder or leaf chewer), diet breadth (host generalist or specialist), and time of sampling (24h or $96 \mathrm{~h}$ ) on expression of LOX2 of Brassica nigra plants. A. Main model selection steps indicating AIC values of the full model (including all fixed factors, their interactions, and all random factors), the null model (assuming all fixed factors equal to zero), and the optimal model (lowest AIC value). We adjusted for species-specific effects by including herbivore identity as random intercept. B. Results from the optimal model (generalized linear mixed model (GLMM) with gamma error distribution and inverse link function). Values in bold denote significant factors $(P<0.05)$.

\begin{tabular}{|c|c|c|c|}
\hline \multicolumn{4}{|l|}{ A. } \\
\hline Model & \multicolumn{3}{|c|}{ Expression } \\
\hline Full & \multicolumn{3}{|c|}{ LOX2 Guild * Specialization * Time + (1| Inducing Herbivore) } \\
\hline Optimal & \multicolumn{3}{|c|}{ LOX2 Guild * Specialization + Time + (1| Inducing Herbivore) } \\
\hline Null & \multicolumn{3}{|c|}{ LOX2 1 + (1| Inducing Herbivore) } \\
\hline \multicolumn{4}{|l|}{ B. } \\
\hline Fixed factor & $\chi^{2}$ & df & $\boldsymbol{P}$ \\
\hline Guild & 22.952 & 1 & $<0.001$ \\
\hline Specialization & 3.101 & 1 & 0.078 \\
\hline Time & 17.071 & 1 & $<0.001$ \\
\hline Guild * Specialization & 4.5 & 1 & 0.034 \\
\hline
\end{tabular}


Table S5. Effects of the inducing herbivore's identity and time of sampling ( $24 \mathrm{~h}$ or $96 \mathrm{~h}$ ) on expression of PR1 of Brassica nigra plants. Main model selection steps indicating AIC values of the full model (including all factors and their interaction) and the null model (assuming all factors equal to zero). The null model (generalized linear model (GLM) with gamma error distribution and log link function) resulted in lowest AIC score.

\begin{tabular}{lll}
\hline Model & Expression & AIC \\
\hline Full & $P R 1 \sim$ Inducing Herbivore * Time & 726 \\
\hline Null & $P R 1 \sim 1$ & 697 \\
\hline
\end{tabular}

Table S6. Effects of herbivore feeding guild (phloem feeder or leaf chewer), diet breadth (host generalist or specialist), and time of sampling (24h or $96 \mathrm{~h}$ ) on expression of PR1 of Brassica nigra plants. A. Main model selection steps indicating AIC values of the full model (including all fixed factors and their interactions, and all random factors), the null model (assuming all fixed factors equal to zero), and the optimal model (lowest AIC value). We adjusted for species specific effects by including the herbivore identity as random intercept. B. Results from the optimal model (generalized linear mixed model (GLMM) with gamma distribution and inverse link function). Values in bold denote significant factors $(P<0.05)$.

\begin{tabular}{llc}
\hline A. & & \\
\hline Model & Expression & AIC \\
\hline Full & $P R 1 \sim$ Guild * Specialization * Time + (1 | Inducing Herbivore $)$ & 510 \\
\hline Optimal & $P R 1 \sim$ Guild * Time + Specialization + (1 | Inducing Herbivore $)$ & 505 \\
\hline Null & $P R 1 \sim 1+(1 \mid$ Inducing Herbivore $)$ & 508 \\
\hline
\end{tabular}

\begin{tabular}{llll}
\hline B. & & & \\
\hline Fixed factor & $\boldsymbol{\chi}^{\mathbf{2}}$ & df & $\boldsymbol{P}$ \\
\hline Guild & 1.954 & 1 & 0.162 \\
\hline Specialization & 2.455 & 1 & 0.937 \\
\hline Time & 0.006 & 1 & 0.117 \\
\hline Guild * Time & 7.021 & 1 & $\mathbf{0 . 0 0 8}$ \\
\hline
\end{tabular}


Table S7. Effects of the inducing and receiving herbivore species' identities on performance (LnRR) of the receiving herbivore in our greenhouse experiments. A. Main model selection steps indicating AIC values of the full model (including all fixed factors and their interactions, and all random factors) and the null model (assuming all fixed factors equal to zero). We adjusted for dependency of observations by including plant identity (Plant ID) as random intercept. B. Results from the full model (linear mixed effect model (LMM)). Values in bold denote significant factors $(P<0.05)$.

\begin{tabular}{llll}
\hline A. & & \\
\hline Model & Expression & \\
\hline Full & LnRR Inducing Herbivore * Receiving Herbivore + (1 | Plant ID $)$ \\
\hline Null & LnRR $\sim$ (1 $\mid$ Plant ID $)$ & \\
\hline B. & & df & $\mathbf{P}$ \\
\hline Fixed factor & $\boldsymbol{F}$ & 9 & $<\mathbf{0 . 0 0 1}$ \\
\hline Inducing Herbivore & 4.189 & 9 & $<\mathbf{0 . 0 0 1}$ \\
\hline Receiving Herbivore & 58.637 & 81 & \\
\hline $\begin{array}{l}\text { Inducing Herbivore * } \\
\text { Receiving Herbivore }\end{array}$ & 3.985 & & \\
\hline
\end{tabular}


Table S8. Performance of herbivores on Brassica nigra plants induced by different herbivores in a greenhouse experiment. To allow comparison across multiple receiving herbivore species, we transformed the data by taking the natural logarithm of the ratio between the performance (number of aphids, or larval weight) of herbivores on induced plants and its mean performance on uninfested control plants (LnRR). Performance for each pairwise interaction is estimated by applying a linear mixed effect model (LMM). Interaction frequency represents the percentage of $B$. nigra plants in the field experiments on which the inducing herbivore species was observed before or at the same time as the receiving herbivore species. Two interactions were excluded (Mpn - Mp and Ar - Ag; annotated with an asterisk) due to uncertainty about the data and extremely low recapture rates respectively. Abbreviations: Ag, Autographa gamma; Ar, Athalia rosae; Bb, Brevicoryne brassicae; Le, Lipaphis erysimi; Mb, Mamestra brassicae; Mp, Myzus persicae; Mpn, Myzus persicae sub. nicotianae; $\mathrm{Pb}$, Pieris brassicae; $\mathrm{Pr}$, Pieris rapae; Px, Plutella xylostella.

\begin{tabular}{|c|c|c|c|c|}
\hline Inducer & Receiver & $\begin{array}{c}\text { Mean performance } \\
\text { (LnRR) }\end{array}$ & SE & $\begin{array}{l}\text { Interaction frequency } \\
\text { (\% of plants) }\end{array}$ \\
\hline Px & $\mathrm{Bb}$ & -0.2876 & 0.0729 & 52.25 \\
\hline $\mathrm{Mp}$ & Px & -0.1575 & 0.0432 & 50.41 \\
\hline Px & $\mathrm{Mp}$ & 0.3764 & 0.0775 & 45.08 \\
\hline $\mathrm{Mp}$ & $\operatorname{Pr}$ & -0.4356 & 0.0939 & 43.65 \\
\hline Px & $\mathrm{Pr}$ & -0.0270 & 0.0758 & 43.44 \\
\hline $\mathrm{Mp}$ & $\mathrm{Bb}$ & -0.3289 & 0.0729 & 40.57 \\
\hline $\mathrm{Bb}$ & Px & -0.0781 & 0.0400 & 38.11 \\
\hline $\mathrm{Bb}$ & $\mathrm{Mp}$ & 0.1931 & 0.0879 & 34.63 \\
\hline $\mathrm{Bb}$ & $\operatorname{Pr}$ & -0.3269 & 0.1217 & 29.92 \\
\hline $\operatorname{Pr}$ & $\mathrm{Mp}$ & 0.2014 & 0.0949 & 27.25 \\
\hline $\operatorname{Pr}$ & Px & -0.2708 & 0.0398 & 26.84 \\
\hline $\operatorname{Pr}$ & $\mathrm{Bb}$ & -0.0758 & 0.0691 & 24.18 \\
\hline $\mathrm{Mb}$ & $\mathrm{Pr}$ & -0.3633 & 0.0902 & 23.57 \\
\hline $\mathrm{Mp}$ & $\mathrm{Mb}$ & 0.2240 & 0.0597 & 22.13 \\
\hline Px & $\mathrm{Mb}$ & 0.1260 & 0.0520 & 20.90 \\
\hline $\mathrm{Mb}$ & $\mathrm{Bb}$ & -0.2550 & 0.0691 & 20.29 \\
\hline $\mathrm{Mb}$ & Px & -0.1671 & 0.0409 & 19.06 \\
\hline $\mathrm{Mb}$ & $\mathrm{Mp}$ & 0.0284 & 0.0822 & 18.85 \\
\hline Le & $\mathrm{Bb}$ & -0.2777 & 0.0691 & 14.14 \\
\hline Le & Px & -0.0935 & 0.0406 & 13.32 \\
\hline
\end{tabular}


Table S8. Continued.

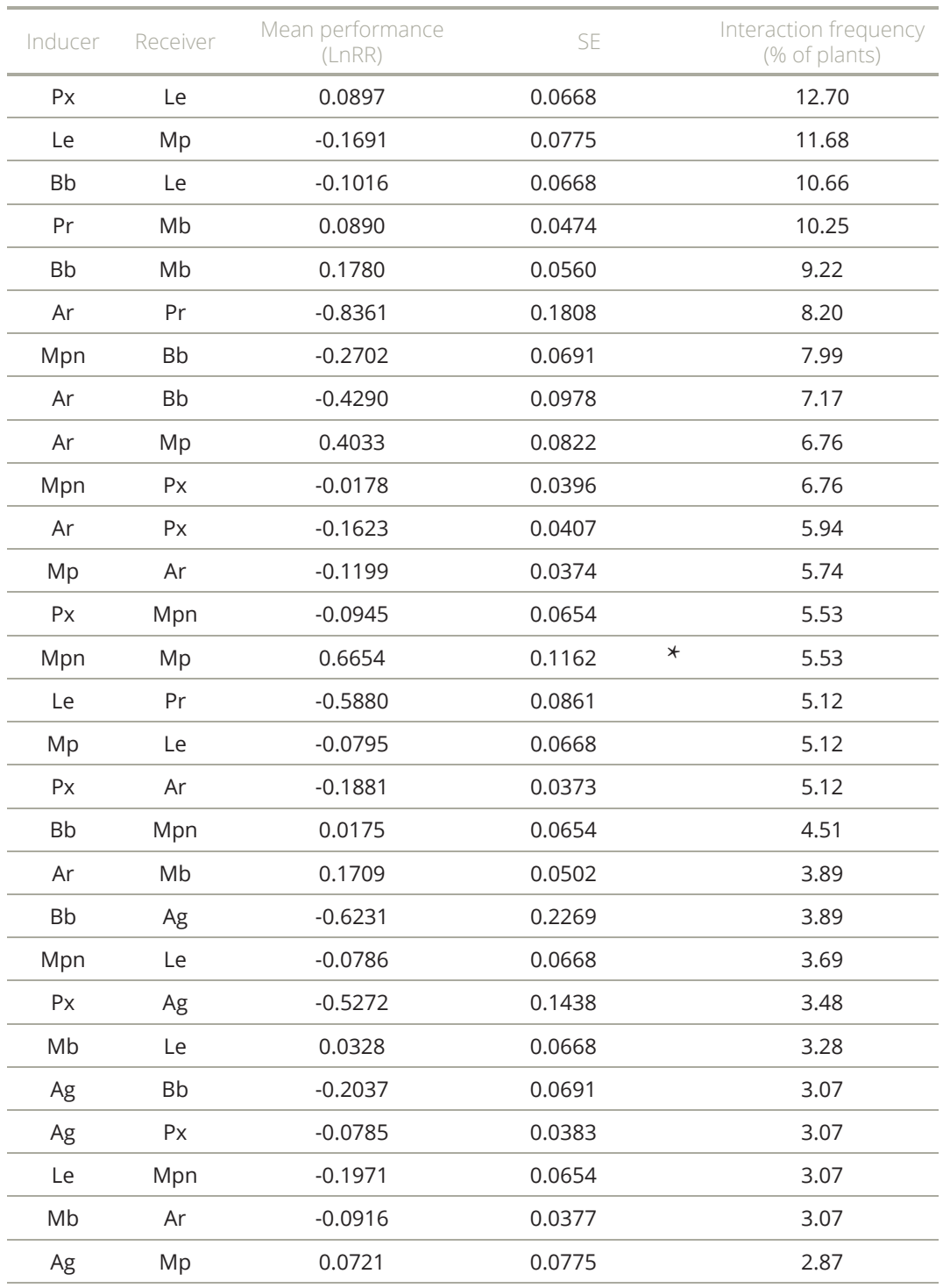


Table S8. Continued.

\begin{tabular}{|c|c|c|c|c|}
\hline Inducer & Receiver & $\begin{array}{c}\text { Mean performance } \\
\text { (LnRR) }\end{array}$ & SE & $\begin{array}{c}\text { Interaction frequency } \\
\text { ( } \% \text { of plants) }\end{array}$ \\
\hline Le & $\mathrm{Mb}$ & 0.0748 & 0.0554 & 2.87 \\
\hline $\mathrm{Mp}$ & $\mathrm{Ag}$ & -0.6730 & 0.1319 & 2.87 \\
\hline $\mathrm{Bb}$ & $\mathrm{Ar}$ & -0.1128 & 0.0380 & 2.66 \\
\hline $\operatorname{Pr}$ & $\mathrm{Ar}$ & -0.1209 & 0.0382 & 2.66 \\
\hline $\mathrm{Mp}$ & Mpn & -0.0850 & 0.0654 & 2.66 \\
\hline $\mathrm{Mb}$ & Mpn & -0.0354 & 0.0690 & 2.05 \\
\hline $\operatorname{Pr}$ & Le & -0.1176 & 0.0668 & 2.05 \\
\hline $\operatorname{Pr}$ & Mpn & -0.0942 & 0.0654 & 2.05 \\
\hline Mpn & $\mathrm{Pr}$ & -0.0389 & 0.0677 & 1.84 \\
\hline $\mathrm{Ag}$ & Le & -0.0923 & 0.0668 & 1.64 \\
\hline Le & $\mathrm{Ag}$ & -0.6313 & 0.2025 & 1.64 \\
\hline $\mathrm{Pb}$ & $\mathrm{Mp}$ & 0.2110 & 0.0775 & 1.64 \\
\hline $\mathrm{Pb}$ & Px & -0.2200 & 0.0416 & 1.64 \\
\hline $\operatorname{Pr}$ & $\mathrm{Ag}$ & -0.1648 & 0.2630 & 1.64 \\
\hline $\mathrm{Pb}$ & $\mathrm{Bb}$ & -0.3210 & 0.0826 & 1.43 \\
\hline Mpn & $\mathrm{Mb}$ & 0.3184 & 0.0533 & 1.23 \\
\hline $\mathrm{Bb}$ & $\mathrm{Pb}$ & -0.0487 & 0.0363 & 1.02 \\
\hline Le & $\mathrm{Ar}$ & 0.0144 & 0.0381 & 1.02 \\
\hline $\mathrm{Mb}$ & $\mathrm{Ag}$ & -0.2315 & 0.1604 & 1.02 \\
\hline $\mathrm{Ag}$ & $\mathrm{Pr}$ & -0.2777 & 0.1062 & 0.82 \\
\hline $\mathrm{Ar}$ & Le & -0.0852 & 0.0668 & 0.82 \\
\hline Le & $\mathrm{Pb}$ & 0.0054 & 0.0343 & 0.82 \\
\hline Mpn & $\mathrm{Ag}$ & -0.5998 & 0.1832 & 0.82 \\
\hline Px & $\mathrm{Pb}$ & -0.0219 & 0.0334 & 0.82 \\
\hline $\mathrm{Ag}$ & $\mathrm{Mb}$ & 0.2419 & 0.0595 & 0.61 \\
\hline $\mathrm{Ag}$ & Mpn & -0.1140 & 0.0654 & 0.61 \\
\hline $\mathrm{Pb}$ & $\mathrm{Mb}$ & 0.3155 & 0.0488 & 0.61 \\
\hline $\mathrm{Pb}$ & $\operatorname{Pr}$ & -0.1334 & 0.0841 & 0.61 \\
\hline
\end{tabular}


Table S8. Continued.

\begin{tabular}{ccccc}
\hline Inducer & Receiver & $\begin{array}{c}\text { Mean performance } \\
(\text { LnRR) }\end{array}$ & SE & $\begin{array}{c}\text { Interaction frequency } \\
\text { (\% of plants) }\end{array}$ \\
\hline $\mathrm{Mp}$ & $\mathrm{Pb}$ & -0.0341 & 0.0364 & 0.41 \\
\hline $\mathrm{Mpn}$ & $\mathrm{Ar}$ & -0.0892 & 0.0392 & 0.41 \\
\hline $\mathrm{Mpn}$ & $\mathrm{Pb}$ & -0.0051 & 0.0336 & 0.41 \\
\hline $\mathrm{Pb}$ & $\mathrm{Le}$ & -0.0838 & 0.0668 & 0.41 \\
\hline $\mathrm{Pr}$ & $\mathrm{Pb}$ & -0.0801 & 0.0339 & 0.41 \\
\hline $\mathrm{Ag}$ & $\mathrm{Ar}$ & -0.0915 & 0.0388 & 0.20 \\
\hline $\mathrm{Ar}$ & $\mathrm{Mpn}$ & -0.2575 & 0.0654 & 0.20 \\
\hline $\mathrm{Ar}$ & $\mathrm{Pb}$ & 0.1402 & 0.0344 & 0.20 \\
\hline $\mathrm{Mb}$ & $\mathrm{Pb}$ & -0.0858 & 0.0336 & 0.20 \\
\hline $\mathrm{Ag}$ & $\mathrm{Pb}$ & -0.0022 & 0.0342 & 0.00 \\
\hline $\mathrm{Pb}$ & $\mathrm{Ag}$ & -0.2666 & 0.1329 & 0.00 \\
\hline $\mathrm{Pb}$ & $\mathrm{Ar}$ & -0.0151 & 0.0368 & 0.00 \\
\hline $\mathrm{Pb}$ & $\mathrm{Mpn}$ & -0.2393 & 0.0654 & 0.00 \\
\hline $\mathrm{Ar}$ & $\mathrm{Ag}$ & -0.7962 & - & 0.00 \\
\hline
\end{tabular}




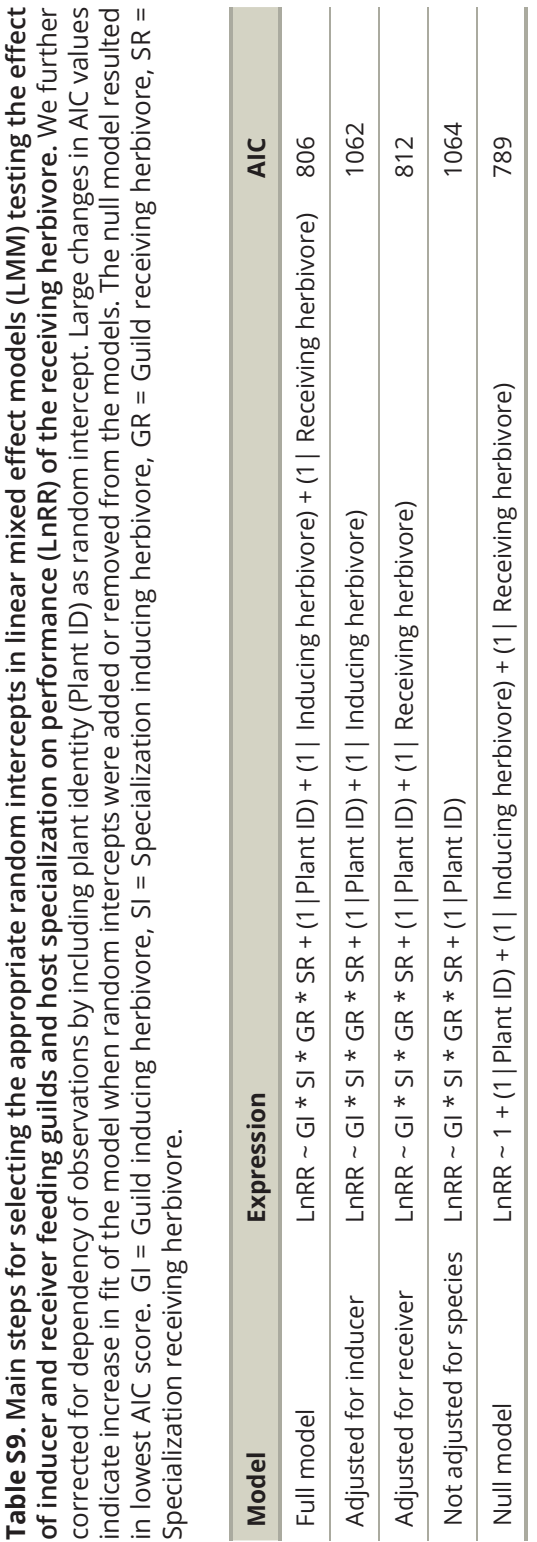


Table S10. Effects of the inducing and receiving herbivore feeding guilds and host specialization on performance (LnRR) of the receiving herbivore, not adjusted for species identities. A. Main model selection steps indicating AIC values of the full model (including all fixed factors and their interactions, and all random factors) and the null model (assuming all fixed factors equal to zero). We adjusted for dependency of observations by including plant identity (Plant ID) as random intercept. We accounted for heterogeneity of variance by allowing different variance for the different levels of the inducer guild, and specialisation of the receiving herbivore. $\mathrm{Gl}=$ Guild inducing herbivore, $\mathrm{SI}=$ Specialization inducing herbivore, $\mathrm{GR}=$ Guild receiving herbivore, $\mathrm{SR}=$ Specialization receiving herbivore. B. Results from the optimal model (linear mixed effect model $(\mathrm{LMM}))$. Values in bold denote significant factors $(P<0.05)$.

\begin{tabular}{lll}
\hline A. & & \\
\hline Model & Expression & AIC \\
\hline Full & LnRR $\sim \mathrm{Gl} * \mathrm{SI} * \mathrm{GR} * \mathrm{SR}+(1 \mid$ Plant ID $)$ & 1098 \\
\hline Optimal & LnRR $\sim \mathrm{Gl} * \mathrm{SR}+(1 \mid$ Plant ID $)$ & 1032 \\
\hline Null & LnRR $\sim 1+(1 \mid$ Plant ID $)$ & 1058 \\
\hline
\end{tabular}

\begin{tabular}{|c|c|c|c|}
\hline \multicolumn{4}{|l|}{ B. } \\
\hline Fixed factor & $\chi^{2}$ & df & $P$ \\
\hline Guild inducing herbivore & 0.9910 & 9 & 0.319 \\
\hline Specialisation receiving herbivore & 44.7509 & 9 & $<0.001$ \\
\hline $\begin{array}{l}\text { Guild inducing herbivore * Specialisation } \\
\text { receiving herbivore }\end{array}$ & 3.3179 & 81 & $<0.069$ \\
\hline
\end{tabular}


Table S11. Effects of the inducer and receiver feeding guilds, host specialization, and prevalence in the field on performance (LnRR) of the receiving herbivore. We further corrected for dependency of observations by including plant identity (Plant ID) as random intercept. A. Main model selection steps indicating AIC values of the null model (assuming all fixed factors equal to zero), the optimal model, and an overview of the contribution in terms of AIC of fixed factors to the model fit. The full model including all explanatory factors did not converge due to overfitting. GI = Guild inducing herbivore, $\mathrm{SI}=$ Specialization inducing herbivore, $\mathrm{PI}=$ Prevalence inducing herbivore, $\mathrm{GR}=$ Guild receiving herbivore, $\mathrm{SR}=$ Specialization receiving herbivore, $\mathrm{PR}=$ Prevalence receiving herbivore. We accounted for heterogeneity of variance by allowing different variance for the different levels of feeding guild in the inducing herbivore, and by allowing variance to exponentially increase with increasing receiver prevalence. B. Results from the optimal model. Values in bold denote significant factors $(P<0.05)$.

\begin{tabular}{|c|c|c|}
\hline \multicolumn{3}{|l|}{ A. } \\
\hline Model & Expression & AIC \\
\hline Null model & $\operatorname{LnRR} \sim 1$ & 565 \\
\hline Optimal model & $\mathrm{LnRR} \sim \mathrm{GR} * \mathrm{PI}+\mathrm{SR} * \mathrm{GR}$ * RP $+\mathrm{PR}$ *PI * SI & 293 \\
\hline $\begin{array}{l}\text { - Interaction } \\
(\mathrm{GR} * \mathrm{PI})\end{array}$ & $\mathrm{LnRR} \sim \mathrm{SR}$ * GR * RP + PR * PI * SI & 308 \\
\hline $\begin{array}{l}\text { - Interaction } \\
(\mathrm{SR} * \mathrm{GR} * \mathrm{RP})\end{array}$ & 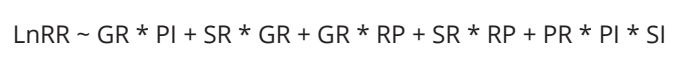 & 349 \\
\hline $\begin{array}{l}\text { - Interaction } \\
(\mathrm{PR} * \mathrm{PI} \text { * SI) }\end{array}$ & $\mathrm{LnRR} \sim \mathrm{GR}$ * $\mathrm{PI}+\mathrm{SR}$ * GR * RP $+\mathrm{PR}$ * $\mathrm{PI}+\mathrm{PI}$ * SI $+\mathrm{PR}$ * SI & 297 \\
\hline
\end{tabular}

\begin{tabular}{llll}
\hline B. & & & \\
Fixed factor & $\boldsymbol{\chi}^{\mathbf{2}}$ & df & $\boldsymbol{P}$ \\
\hline $\mathrm{GR} * \mathrm{PI}$ & 16.9702 & 1 & $<\mathbf{0 . 0 0 1}$ \\
\hline $\mathrm{SR} * \mathrm{GR} * \mathrm{PR}$ & 59.9579 & 1 & $<\mathbf{0 . 0 0 1}$ \\
\hline $\mathrm{PR} * \mathrm{PI} * \mathrm{SI}$ & 6.5973 & 1 & $\mathbf{0 . 0 1 0}$ \\
\hline
\end{tabular}




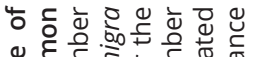
ษ $\varepsilon$ है

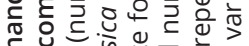

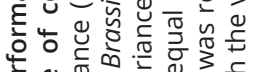

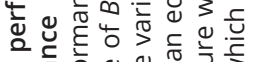

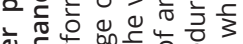

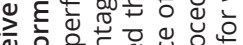

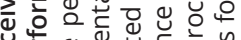

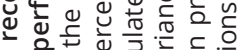
$\subseteq \simeq 4$ 造部

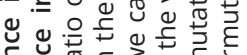
元宁它

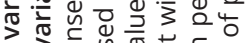

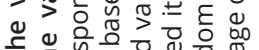
뽛

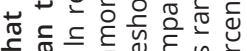

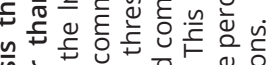

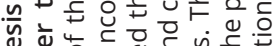

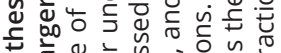

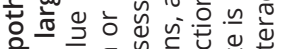

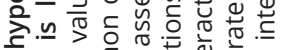

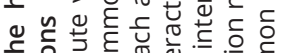

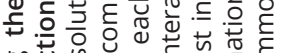

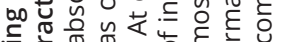

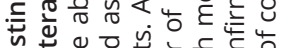

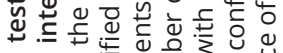

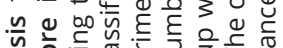

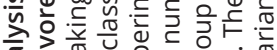

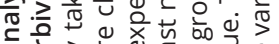

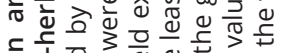

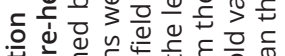

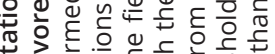

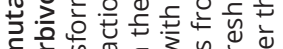

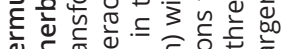

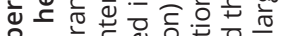
으의

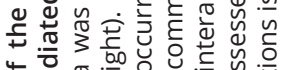
就

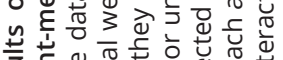

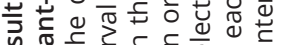

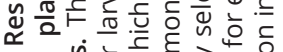

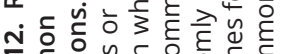

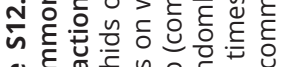

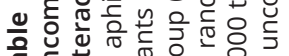

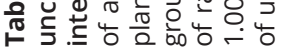

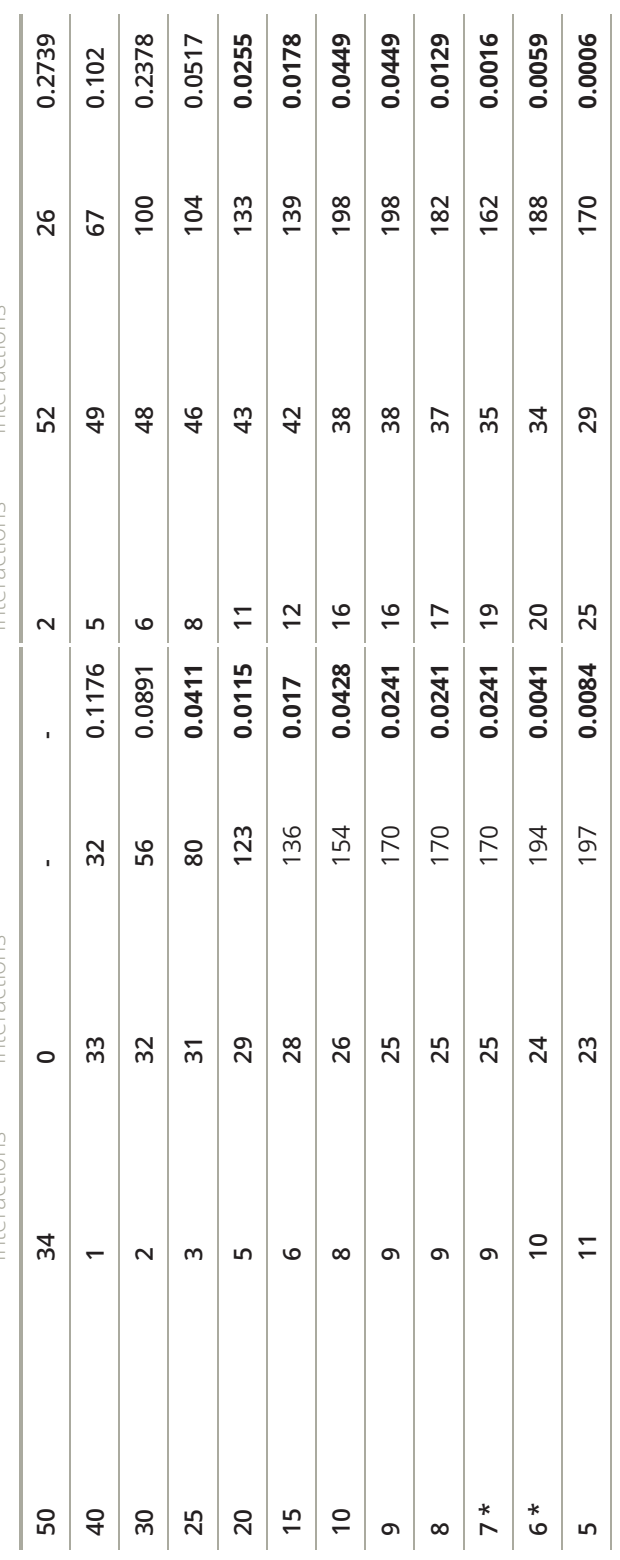




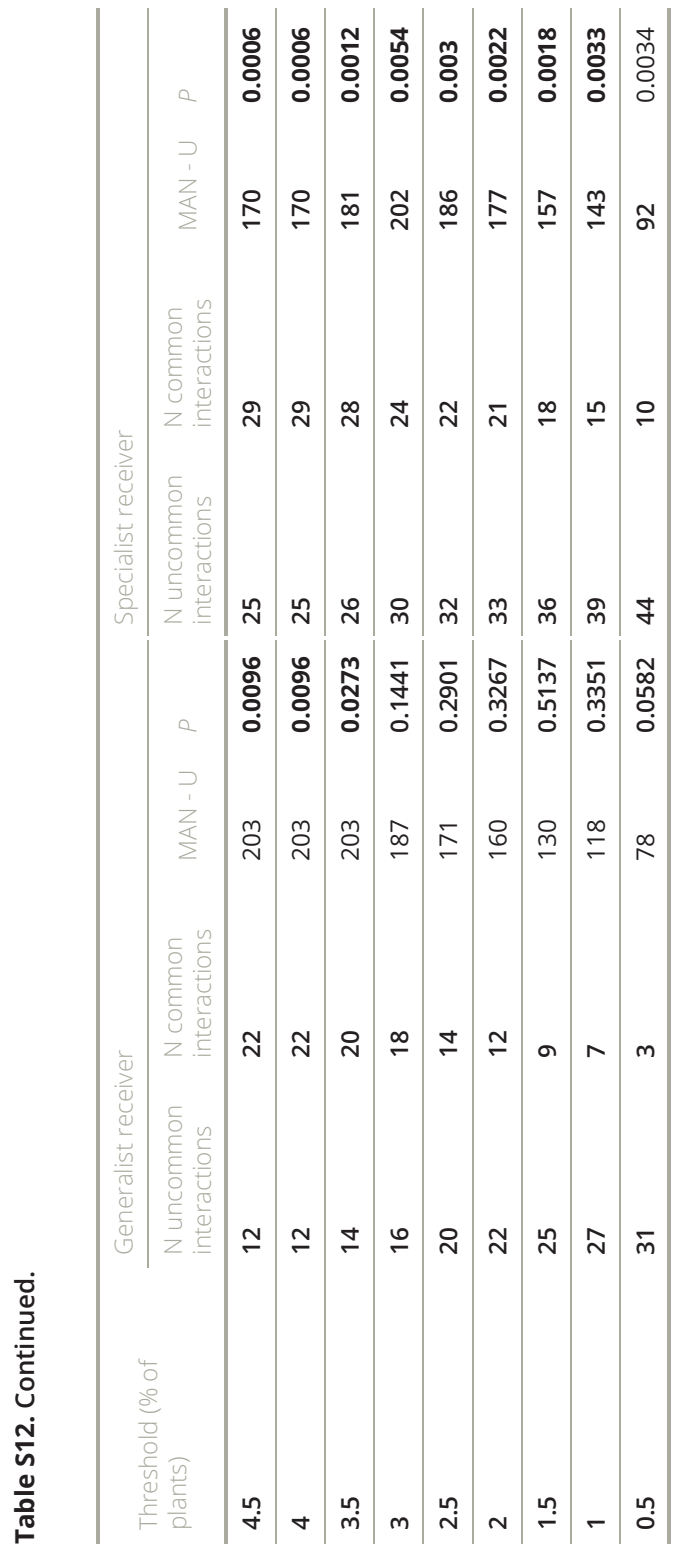


Table S13. Results of the permutation analysis testing the hypothesis that the variance in receiver performance of uncommon plant-mediated herbivore-herbivore interactions is larger than the variance in performance of common interactions. The data was transformed by taking the absolute value of the In response ratio of the performance (number of aphids or larval weight). Interactions were classified as common or uncommon based on the percentage of Brassica nigra plants on which they occurred in the field experiments. At each assessed threshold value, we calculated the variance for the group (common or uncommon) with the least number of interactions, and compared it with the variance of an equal number of randomly selected interactions from the group with most interactions. This random permutation procedure was repeated 1.000 times for each assessed threshold value. The confirmation rate is the percentage of permutations for which the variance of uncommon interactions is larger than the variance of common interactions.

\begin{tabular}{|c|c|c|c|c|c|}
\hline $\begin{array}{l}\text { Threshold } \\
\text { ( } \% \text { of } \\
\text { plants) }\end{array}$ & $\begin{array}{c}\mathrm{N} \\
\text { uncommon } \\
\text { interactions }\end{array}$ & $\begin{array}{l}\text { Estimated variance } \\
\text { uncommon } \\
\text { interactions }\end{array}$ & $\begin{array}{l}\mathrm{N} \text { common } \\
\text { interactions }\end{array}$ & $\begin{array}{c}\text { Estimated } \\
\text { variance common } \\
\text { interactions }\end{array}$ & $\begin{array}{c}\text { Confirmation } \\
\text { rate }(\%)\end{array}$ \\
\hline 50 & 2 & 0.0274 & 86 & 0.0299 & 50.5 \\
\hline 40 & 6 & 0.0310 & 82 & 0.0296 & 47.1 \\
\hline 30 & 8 & 0.0301 & 80 & 0.0302 & 57.5 \\
\hline 25 & 11 & 0.0311 & 77 & 0.0311 & 74.1 \\
\hline 20 & 16 & 0.0331 & 72 & 0.0325 & 88.7 \\
\hline 15 & 18 & 0.0333 & 70 & 0.0331 & 91.4 \\
\hline 10 & 24 & 0.0352 & 64 & 0.0350 & 97.9 \\
\hline 9 & 25 & 0.0363 & 63 & 0.0361 & 98.8 \\
\hline 8 & 26 & 0.0301 & 62 & 0.0298 & 61.7 \\
\hline 7 & 28 & 0.0297 & 60 & 0.0296 & 63.7 \\
\hline 6 & 30 & 0.0293 & 58 & 0.0293 & 58.1 \\
\hline 5 & 36 & 0.0293 & 52 & 0.0290 & 55.8 \\
\hline 4.5 & 37 & 0.0292 & 51 & 0.0291 & 57.0 \\
\hline 4 & 37 & 0.0292 & 51 & 0.0291 & 56.4 \\
\hline 3.5 & 40 & 0.0262 & 48 & 0.0264 & 0.00 \\
\hline 3 & 46 & 0.0263 & 42 & 0.0312 & 10.1 \\
\hline 2.5 & 52 & 0.0225 & 36 & 0.0334 & 6.90 \\
\hline 2 & 55 & 0.0239 & 33 & 0.0327 & 15.9 \\
\hline 1.5 & 61 & 0.0199 & 27 & 0.0325 & 10.8 \\
\hline 1 & 66 & 0.0202 & 22 & 0.0316 & 19.7 \\
\hline 0.5 & 75 & 0.0087 & 13 & 0.0316 & 5.50 \\
\hline
\end{tabular}




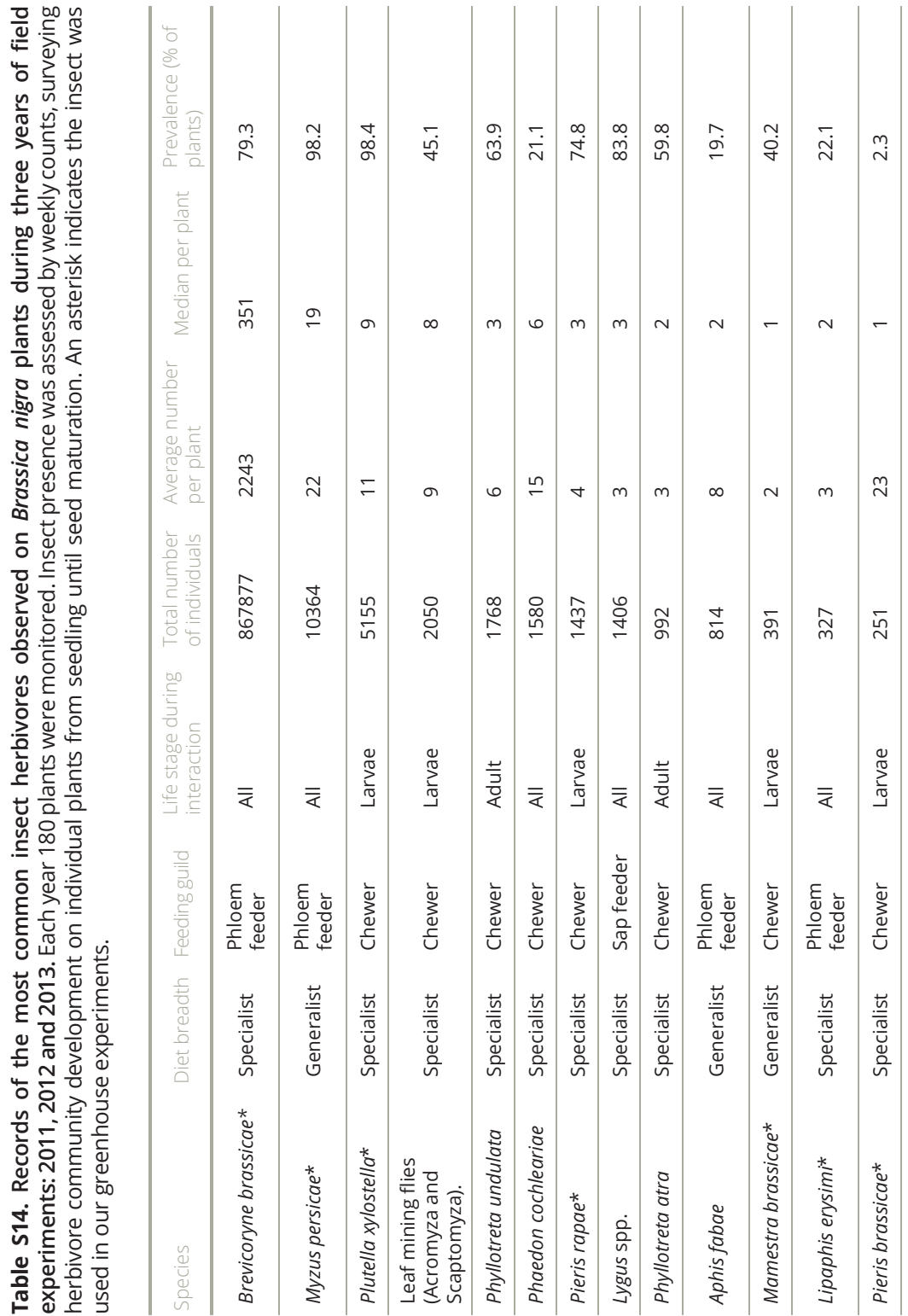




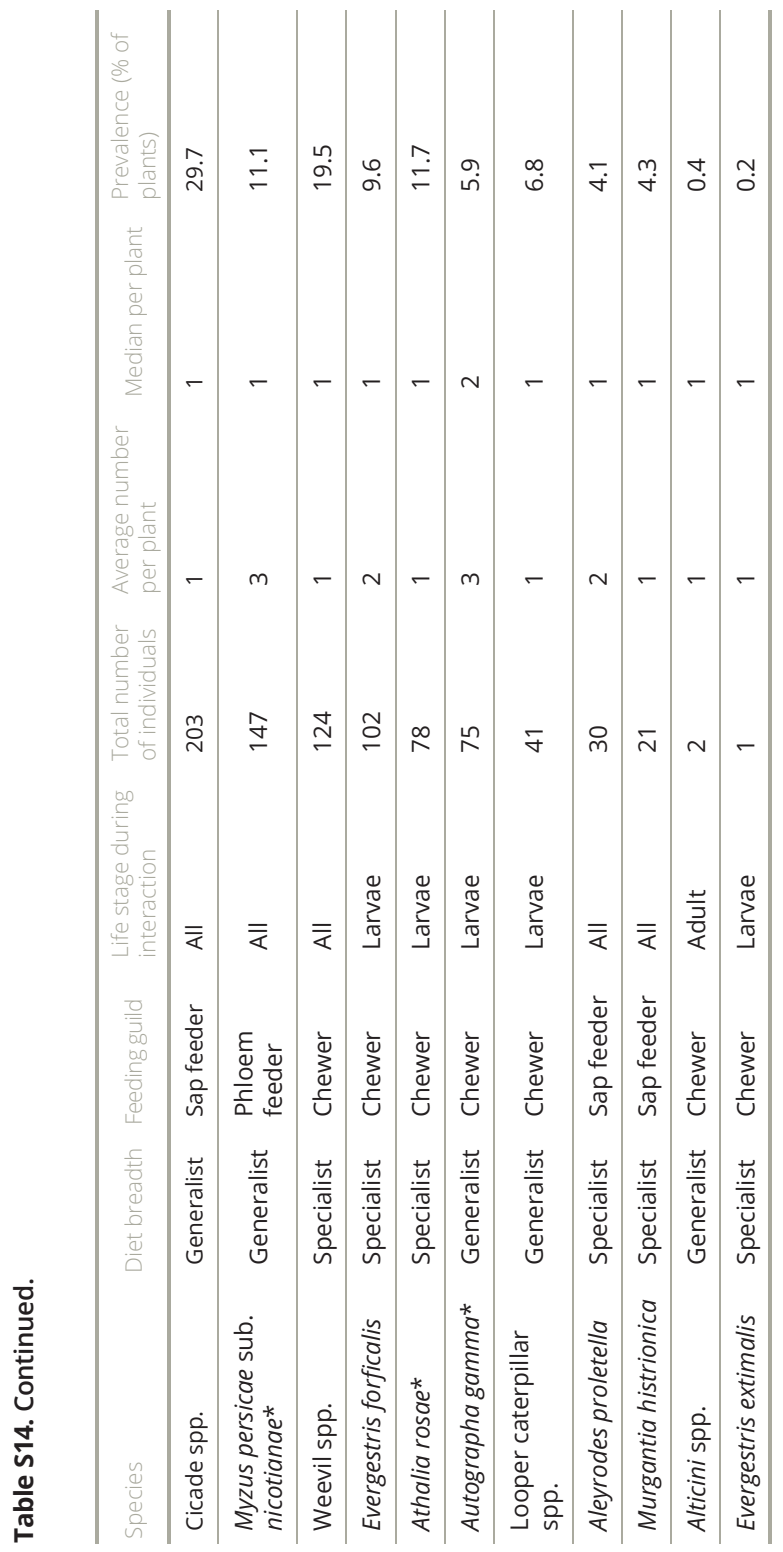




\section{SUPPLEMENTARY RESULTS AND DISCUSSION}

\section{Plant transcriptional responses to herbivore induction}

The relative expression of the JA-marker gene LOX2 strongly depended on the inducing herbivore species (Table S3 and S4). Treatment effects ranged from almost no effect up to an 8-fold increase in transcription of LOX2 compared to expression in non-treated plants (Extended Data Figure 2A). We observed an increase in relative expression of LOX2 over time, which was included as an additive effect in the optimal model (Table S3). When relating relative expression of LOX2 to the feeding behaviour of the herbivore, we found that it depended on the interaction between feeding guild and host specialization of the herbivore with an additive effect of the time after the start of herbivory (Extended Data Figure 2B). At 24 hours since start of herbivory, specialist leaf chewers induced a higher expression of $L O X 2$ compared to generalist leaf chewers or phloem feeders (Tukey HSD; $P=0.007 ; P=0.003$ respectively). At 96 hours after herbivory, specialist leaf chewers induced a stronger LOX2 expression level than specialist phloem feeders (Tukey HSD; $P=0.042$ ), while marginally insignificant when compared to generalist phloem feeders (Tukey HSD; $P=0.058)$. Overall, our findings show that chewing herbivores induce a stronger response of this JA marker gene compared to phloem feeders.

We found no herbivore-specific induction of the SA-marker gene PR1 (Extended Data Figure 2C; Table S5). Expression of PR1 could best be explained by an interaction between the herbivore's feeding guild and the time after herbivory, and an additive effect of the herbivore's host specialization. It should however be noted that this model was not considered significantly better than the null model $\left(\right.$ Table S6; AIC $\left._{\text {Full }}=511 ; \mathrm{AIC}_{\text {Optimal }}=506 ; \mathrm{AIC}_{\text {Null }}=508\right)$, indicating that effects are likely to be small. Indeed, post hoc analysis of the optimal model showed only a significantly higher expression of PR1 at 96 hours in generalist or specialist phloem feeder treatments compared to treatments involving generalist chewers (Tukey HSD; $P=0.050 ; P=0.038$ respectively) (Extended Data Figure 2D). Hence, this analysis suggests that phloem feeders induce higher transcription of PR1 in $B$. nigra compared to leaf chewers, although differences in the induced changes in 
expression levels are minor. Overall, the results confirm that leaf chewers induce stronger plant responses characterized by a gene that regulates the JA signal transduction pathway, whereas plant responses to aphids induced stronger plants responses characterized by a gene that is downstream on the SA signal transduction pathway. For chewing herbivores, induction of a JA-related gene LOX2 was stronger in response to attack by specialists compared to attack by generalists.

\section{Herbivore performance}

Performance of the receiver species feeding on plants that were previously induced by one of the ten herbivore species (Table S1) strongly depended on the interaction between the identity of the inducing and the receiving herbivore species (Table S7). This suggests that the outcome of plant-mediated pairwise interactions was highly species-specific (Fig. 1A, Extended Data Figure 2, Table S8). Furthermore, the lack of consistent effects of inducers on receiver performance was corroborated by finding no relationship between the performance of secondary herbivores and the plants transcriptional response (in terms of LOX2 and PR1 expression at 96h) (Extended Data Figure 4). Thus, the induced resistance phenotype of the plant as characterized by specific marker genes had little predictive value for the ecological outcome of pairwise interactions, which is mainly determined by the identity of both inducing and receiving herbivores. In Brassica nigra, herbivore species may specifically induce changes in primary and secondary metabolites as well as morphological traits such as trichomes. Root herbivores, leaf chewing herbivores and phloem feeders have been found to induce an increase in glucosinolates (van Dam et al., 2006; Ponzio, et al., 2017; Papazian et al., 2019; Hopkins et al., 2009), the main class of secondary metabolites involved in herbivore resistance of Brassicaceae (Hopkins et al., 2009). Phloem feeders and leaf chewers have been found to differ in their effect on primary metabolite profiles in leaves, such as sugar pools (Ponzio, et al., 2017; Papazian et al., 2019). Specialist and generalist herbivores may experience reduced performance on herbivore-induced B. nigra plants and the impact of specific defence traits may be herbivore species specific, with stronger negative effects of glucosinolates on generalists than on specialists (Hopkins et al., 2009; Traw et al., 2006). 
When testing the effects of the functional groups (host specialization and feeding guild) of both the inducing and receiving herbivore on performance of the secondary herbivore, we found that species-specific effects within feeding guild and host specialization of the receiving herbivore were driving our analysis (Table S9). This confirms that species identity of the secondary herbivore is more important in explaining the outcome of plant-mediated interactions among herbivores than the identity of the inducing herbivore. To allow a more general interpretation of our data we proceeded by exploring a model excluding the random species intercepts driving our previous analysis. This new model retained an interaction between feeding guild of the inducer and host specialization of the receiving herbivore that best explained our results (Fig. 1B, Table S10). Generalist receivers performed better on plants induced by chewing herbivores compared to specialist receivers that fed on plants induced by chewers or by phloem feeders (Tukey HSD; $P<0.001$ for both comparisons). In addition, generalist receivers performed better on plants induced by phloem feeders compared to specialist herbivores that fed on plants induced by chewing herbivores (Tukey HSD; $P=0.048$ ). Overall, we found that generalist chewing herbivores performed better on induced plants compared to their performance on non-treated plants, while specialized chewing herbivores performed better on non-treated plants compared to their performance on induced plants. The feeding guild of the inducing herbivore further amplified differences in the outcome of interactions. For generalist herbivores feeding on induced plants, performance was better on plants induced by chewers than by aphids. It is important to note that performance of generalist chewing herbivores was overall poor on both non-treated and induced plants (Extended Data Figure 3). The small absolute differences in performance between undamaged and induced plants as well as the general high mortality of generalist herbivores suggest that these herbivores are strongly negatively affected by constitutive levels of resistance in B. nigra (Hopkins et al., 2009). 


\section{Relation to field observations}

We found that 53 out of the 90 pairwise interactions we tested in our greenhouse experiment were observed on less than $5 \%$ of the plants in the field experiments, and 5 out of 90 interactions were not observed in the field over a three-year period (Fig. 2D, Table S8). Our data show that commonly observed interactions where the second herbivore is a plant specialist are associated with decreasing performance of the receiver relative to its performance on nontreated plants (Fig. 2, Table S12). These common interactions were largely a result of the high prevalence of specialist herbivores (Table S11). The role the prevalence of the receiver had in predicting the outcome of the interactions depended on the specialisation of the receiver and its diet breadth. We found that commonality of the inducer had a small but significant effect on performance of the secondary herbivore depending on the feeding guild of the receiver. Interestingly, this pattern is opposite when the receiver is a generalist. This pattern was robust for a large range of threshold values of what was defined as a common and uncommon interaction (Table S12). Variability in performance was generally lower for interactions we classified as common compared to interactions we classified as uncommon. However, this finding was more sensitive to the choice of the threshold compared to the previous analysis (Table S13). Lower variation in common pairwise-interactions compared to uncommon interactions suggests that uncommon interactions are more unpredictable in terms of receiver performance. These interactions typically consisted of rare secondary herbivores.

We observed 24 herbivore species in our field experiment (Table S14), realizing 453 out of 552 possible pairwise interactions (Fig. 2D). When ranking the observed interactions by the percentage of plants on which they occur (i.e. interaction frequency), we found an exponential decay in prevalence of the interactions. This indicates that a relatively small set of observed interactions occurred on a large percentage of plants while a large proportion of the interactions could be considered uncommon. On a scale from common to uncommon, the pairwise interactions tested in our greenhouse setup represent an 
evenly distributed subset of the 453 interactions observed under field conditions (Fig. 2D), with the exception of five interactions that we did not observe in our field experiment. The interactions tested in our greenhouse experiment that were most common under field conditions involved a subset of only four herbivore species: Plutella xylostella, Myzus persicae, Brevicoryne brassicae, and Pieris rapae (11 interactions occurring on more than $25 \%$ of the plants in our field experiments). These herbivores are characterized by both high prevalence (i.e. \% of plants on which they occur) and abundance in our field experiments (Table S13). The herbivore community developing in our field experiment was increasingly specialist-dominated towards the end of the plants lifetime (Fig. 2C). For chewing herbivores, we found that specialists dominate the community from early stages of community development onward. For phloem feeding herbivores, we observed a generalist-dominated herbivore community early in the season that develops to a specialist-dominated community at the end of the season.

We conclude that plant induced responses to herbivory in B. nigra are adaptive in the context of risk of attack by specialist herbivores later in the season. In addition to food plant specialisation, other traits of herbivores may also impact the evolution of induced defence (Tiffin et al., 2006). Mobility of herbivores has been identified to promote selection on induced plant defences, whereas constitutive defences are effective against immobile herbivores that for longer time during a plant's lifetime reside on plants. Induced defences for less mobile herbivores are only favoured when the specific induced traits are highly effective defences against the specific herbivore and the herbivore feeds for a relatively short time on the plant. Induced defences may also be effective in initiating herbivore movement away from plants, limiting the duration of feeding (Rubin et al., 2015). 



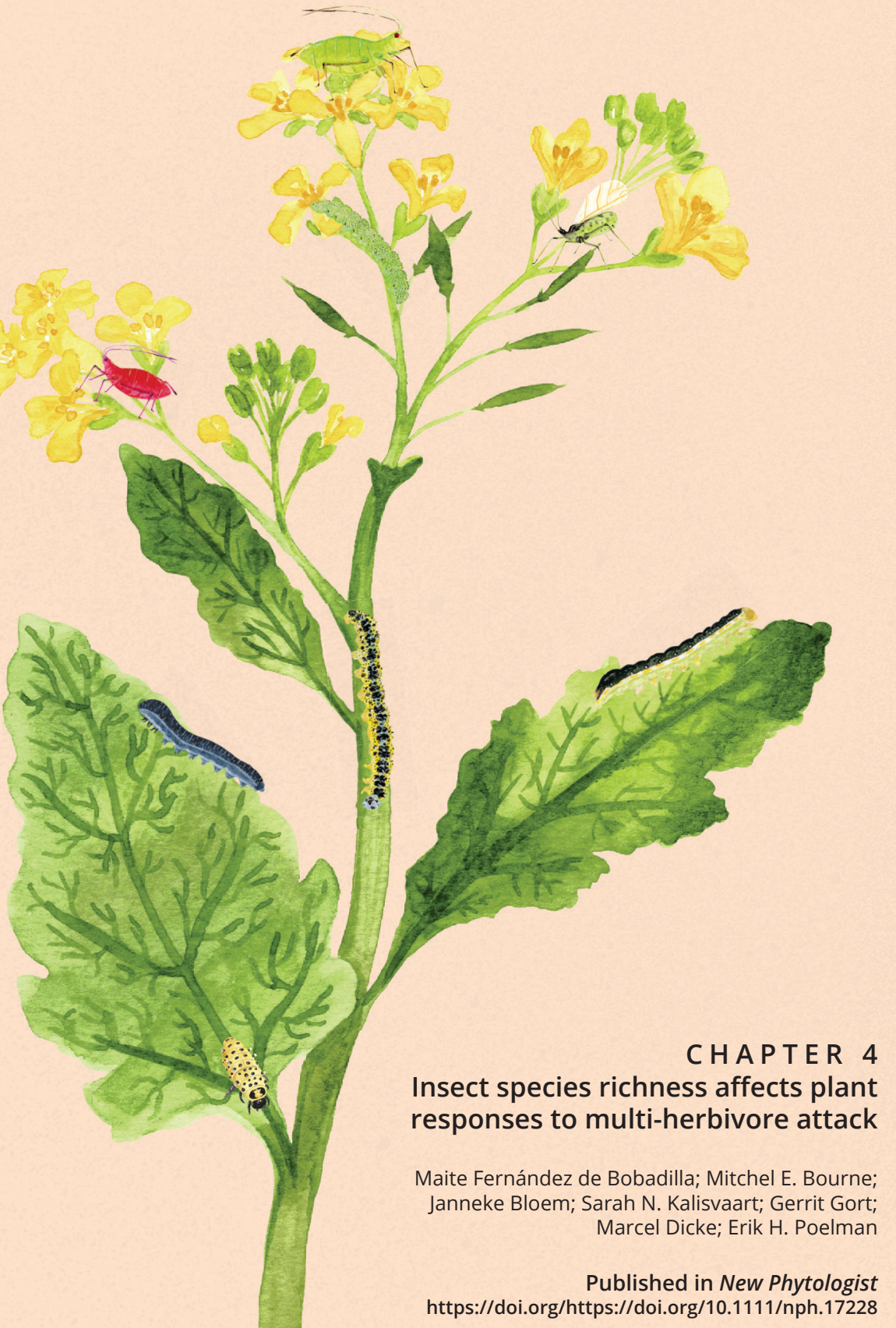





\section{ABSTRACT}

Plants are often attacked by multiple insect herbivores. How plants deal with an increasing richness of attackers from a single or multiple feeding guilds is poorly understood.

We subjected black mustard (Brassica nigra) plants to 51 treatments representing attack by an increasing species richness (1, 2 or 4 species) of either phloem feeders, leaf chewers, or a mix of both feeding guilds when keeping total density of attackers constant and studied how this affects plant resistance to subsequent attack by caterpillars of the diamondback moth (Plutella xylostella).

Increased richness in phloem-feeding attackers compromised resistance to $P$. xylostella. In contrast, leaf chewers induced a stronger resistance to subsequent attack by caterpillars of P. xylostella while species richness did not play a significant role for chewing herbivore induced responses. Attack by a mix of herbivores from different feeding guilds resulted in plant resistance similar to resistance levels of plants that were not previously exposed to herbivory.

We conclude that B. nigra plants channel their defence responses stronger towards a feeding-guild specific response when under multi-species attack by herbivores of the same feeding guild, but integrate responses when simultaneously confronted with a mix of herbivores from different feeding guilds. 


\section{INTRODUCTION}

Plants interact with a community of organisms, from which insects are among the most prominent members. Some of these interactions benefit plant fitness, such as interactions with pollinators or plant growth-promoting microorganisms (Berg, 2009; Pineda et al., 2010; Giron et al., 2018). However, many plant interactions negatively impact plant fitness because they involve organisms that consume plant tissues, such as insect herbivores. In natural ecosystems, plants are commonly attacked by multiple insect herbivores and are under selection to optimise their defences against their community of attackers to maximise their fitness (Lankau \& Strauss, 2008; Wise \& Rausher, 2013; Poelman $\&$ Kessler, 2016). Because the production and maintenance of plant defences is energetically costly, most defence mechanisms are inducible (i.e. only activated upon herbivore feeding) to save the costs of defence in the absence of herbivores (Karban, 2019). To deal with a suite of attackers that may all require different defensive traits, plants have evolved mechanisms to recognize the specific attacker by its feeding guild (e.g. leaf chewer or phloem feeder), feeding pattern, feeding position, or elicitors in its saliva, and tailor the induced defence phenotype to the specific attacking herbivore (Acevedo et al., 2015; Züst \& Agrawal, 2016). In this context, plants use a network of phytohormones to regulate responses to specific herbivores with Jasmonic Acid (JA) signalling being dominantly involved in resistance to leaf chewing herbivores and Salicylic Acid (SA) to phloem feeding herbivores (Walling 2000; Pieterse et al. 2012; Erb \& Reymond, 2019).

When plants are attacked by multiple herbivores, inhibition or potentiation between defence pathways may allow plants to fine tune defence responses to deal with a suite of herbivores (Li et al., 2018; Erb \& Reymond, 2019). However, situations where crosstalk between pathways has an apparent negative effect for the plant may also occur (Koornneef \& Pieterse, 2008; Thaler et al., 2012; Moreira et al., 2018). Phytohormonal crosstalk such as between SA and JA regulatory pathways may impair plant responses to one insect when the plant has already directed its resistance response towards a previous attacker of a different feeding guild (Pieterse et al., 2012; Soler et al., 2012; Moreira et al., 2018). To optimize 
resistance strategies to attack by a suite of herbivores, plants may have to reduce the costs of compromises in resistance to future attack when dealing with the curent attacker. Thus, plants may tailor plasticity in defence to arrival patterns of herbivores in the often species rich antagonist communities they are exposed to (Mertens et al.2021). Yet, little research has addressed plant-herbivore interactions beyond dual attack. The few studies that included a third herbivore have identified that the order of attack by two herbivores and which species are involved in the interaction affect plant resistance to subsequent attack by a third herbivore (Mathur et al., 2013; Stam et al., 2014; Stam et al., 2017; Stam et al., 2019). Many plant species are attacked by an insect community that comprises more than three species, but how plants deal with their common situation of multi-herbivore attack is poorly understood. A meriad of factors may be relevant for plant responses to multiherbivore attack. This includes the variation in herbivore traits such as feeding guild or food plant specialisation, the order of arrival of herbivores and timing of herbivory during plant ontogeny. At the same time, diversity per se or components of diversity may affect species interactions (Loreau \& Hector, 2001; Soliveres et al., 2016). Before disentangling the significance of each of these components, it should be established whether plants do respond differently to attack by a larger number of herbivore species than to a few and whether it matters that these species are more or less similar in traits.

In this study, we tested if species richness of previous attackers affects a plant's capability to respond to a subsequent attacker of the same or of a different feeding guild. We hypothesized that an increasing richness of initial attackers leads to stronger feeding-guild-specific plant responses when all herbivores are of the same feeding guild than when plants are attacked by a mix of herbivores from different feeding guilds. The stronger response to a higher richness of attackers of the same feeding guild is expected to lead to increased resistance to subsequent attack by a herbivore of the same feeding guild, but to increased susceptibility to attack by a herbivore of a different feeding guild. To test these hypotheses, we subjected black mustard (Brassica nigra) plants to attack by an increasing richness (1, 2 or 4 species) of either phloem-feeding, leaf-chewing 
herbivores, or a mix of both. Over twenty different herbivore species may be found on $B$. nigra plants, but over their lifetime individual plants are on average colonised by eight different herbivore species out of this herbivore species pool (Mertens et al., unpublished). We selected eight of the most prevalent herbivore species equally distributed across the leaf chewer and phloem feeder guilds and studied how plant responses to these attackers affect resistance to subsequent attack by caterpillars of the diamondback moth (Plutella xylostella). Caterpillars of P. xylostella typically attack B. nigra later in the season when plants have been previously attacked by other herbivores and virtually all plants in a stand are attacked by P. xylostella (Mertens et al., unpublished). In addition, we quantified how combinations of these herbivores affect the expression of marker genes of the JA- and SA-phytohormonal pathways to characterise induced plant resistance to multi-herbivore attack. By identifying how plants deal with increased species richness of attack, we signal the importance of placing plant defence plasticity in the context of plant-insect community interactions.

\section{MATERIALS AND METHODS}

\section{Plants and insects}

Three-to-four-week-old black mustard plants (B. nigra, Brassicales: Brassicaceae) were used for the experiments. Seeds were obtained from a natural population in the vicinity of Wageningen, The Netherlands ( $51^{\circ} 57^{\prime} 32^{\prime \prime} \mathrm{N}$, $\left.5^{\circ} 40^{\prime} 23^{\prime \prime} \mathrm{E}\right)$. The plants and the insects were cultured in a greenhouse at 22 $\pm 2{ }^{\circ} \mathrm{C}, 60-70 \% \mathrm{RH}$ and 16:8 h L:D photo regime. Eight insect species were used as primary herbivores or inducers of plant defence (Table 1). We used first-instar larvae of herbivores of a leaf-chewing feeding guild: the cabbage moth, Mamestra brassicae ( $M b)$ (Lepidoptera: Noctuidae); the large cabbage white, Pieris brassicae $(\mathrm{Pb})$ (Lepidoptera: Pieridae); the turnip sawfly, Athalia rosae (Ar) (Hymenoptera: Tenthredinidae); and the mustard leaf beetle, Phaedon cochleariae (Pc) (Coleoptera: Chrysomelidae). For members of the phloem-feeding guild we used the cabbage aphid, Brevicoryne brassicae (Bb); the green peach aphid, Myzus persicae $(M p)$; the tobacco aphid, Myzus persicae sub. nicotianae (Mpn); and the mustard aphid, Lipaphis erysimi (Le) (all Hemiptera: Aphididae) (Table 1). 
Table 1. Insects used for the experiment as inducers, in brackets abbreviations used throughout the document.

\begin{tabular}{|c|c|c|c|}
\hline Species (Abbreviation) & Picture & Order & Feeding guild \\
\hline Athalia rosae $(\mathrm{Ar})$ & & Hymenoptera & Leaf chewer \\
Mamestra brassicae $(\mathrm{Mb})$ & & Lepidoptera & Leaf chewer \\
Phaedon cochleariae $(\mathrm{PC})$ & & Coleoptera & Leaf chewer \\
Pieris brassicae $(\mathrm{Pb})$ & & Lepidoptera & Leaf chewer \\
Brevicoryne brassicae $(\mathrm{Bb})$ & & Hemiptera & Phloem feeder \\
Lipaphis erysimi $($ Le) & & Hemiptera & Phloem feeder \\
Myzus persicae $(\mathrm{Mp})$ & & Hemiptera & Phloem feeder \\
Myzus persicae sub. nicotianae $(\mathrm{Mpn})$ & & Phloem feeder \\
\hline
\end{tabular}

As a subsequent herbivore (or receiver), second-instar larvae of the diamondback moth P. xylostella (Lepidoptera: Plutellidae) were used. Larvae of this insect are specialists on brassicaceous plants and feed on foliar tissue, buds and flowers. Mamestra brassicae, P. brassicae, B. brassicae and P. xylostella were reared on Brussels sprouts plants (Brassica oleracea L. var. gemmifera cv. Cyrus). Myzus persicae, $M$. persicae sub. nicotianae, L. erysimi, A. rosae and P. cocbleariae were reared on radish (Raphanus sativus). All insects were obtained from the stock rearing of the Laboratory of Entomology, Wageningen University and were maintained at $22 \pm 2{ }^{\circ} \mathrm{C}, 60-70 \% \mathrm{RH}$ and 16:8 h L:D photo regime.

\section{Induced resistance to $P$. xylostella after multi-herbivore attack}

\section{Species richness effect within feeding guilds}

We first assessed how an increase in richness of attackers affects induced plant resistance to subsequent herbivory by $P$. xylostella caterpillars feeding on induced plants. Due to the large scale of our study and its associated constraints in terms of greenhouse space as well as numbers of caterpillars that can be weighed on a single day, we conducted separate experiments for leaf-chewers 
(CHEW), phloem-feeders (PHLO) and a mix of species of these feeding guilds (MIX). We thus focussed on quantifying the effect of species richness within each feeding guild.

We assessed plant responses to different levels of herbivore richness by infesting each plant with either one (leaf-chewer: CHEW-1; phloem-feeder: PHLO-1), two (CHEW-2; PHLO-2; MIX-2) or four (CHEW-4; PHLO-4; MIX4) herbivore species while keeping total herbivore numbers constant. We excluded richness of three species, because of the imbalance that these treatments would have in number of leaf-chewer and phloem-feeder species. Each experiment included control plants that did not receive any inducing herbivore but were treated in a similar way as plants receiving herbivores (CON-O). The total density used was four leaf chewers and eight phloem feeders for the leafchewer and phloem-feeder experiment respectively, and two leaf chewers plus four phloem feeders for the mixed richness experiment. We chose the number of species and the total density of inducers used based on field observations of insect communities of B. nigra plants (Poelman et al., 2009; Mertens et al., unpublished). We allowed population growth of the initial infestation of the eight adult phloem feeders. For the mixed richness experiment, MIX-2 resulted in 16 insect combinations of a single phloem-feeder and leaf-chewer, that were all tested. For MIX-4 a selection of 12 of the 36 possible combinations of two phloem-feeders and two leaf-chewers were tested, selected to have the biggest differences between species combinations, while each herbivore is present the same number of times (6 times) (Table S1). Thus, while testing the effect of leaf-chewer richness we compared the performance of P. xylostella caterpillars on: 1) control plants (CON-0), 2) plants induced by four larvae of a single chewer species (CHEW-1, for each of the four chewer species), 3) plants induced by two larvae of two chewer species (CHEW-2, for each of the six species combinations), 4) plants induced by four larvae, one of each of the four chewer species (CHEW-4). For the phloem-feeder richness experiment the setup was similar to the leaf-chewer richness, with the only difference that the total number of inducer individuals was eight. For the mixed richness, we compared 
the performance of P. xylostella caterpillars on: 1) control plants (CON-0), 2) plants induced by two larvae of one chewer species plus four phloem feeders of one species (MIX-2, for each of the sixteen species combinations), 3) plants induced by a single larva of two chewer species plus four phloem feeders (two of each species) (MIX-4, for each of the twelve species combinations selected).

Each experiment was divided into two temporal blocks. We prepared a total of ten plant replicates (five per block) per insect combination for the leafchewer and phloem-feeder richness experiment, and eight plant replicates (four per block) per insect combination for the mixed richness experiment (Table 2). To prevent dehydration and cross-contamination between treatments, 4-5 plants receiving the same herbivore combination were placed jointly in trays inundated with water. All treatments with unique species (combinations) replicating the species richness levels were randomized across the greenhouse. Insects were placed on the youngest fully expanded leaf of the plant and could freely feed from the plant for one week (Fig. 1). Movement of herbivores between plants was prevented by water surrounding the pots in the inundated trays. The inducing herbivores were removed with a brush, after seven days of feeding to exclude direct effects of inducing herbivores on the receiver herbivore. One

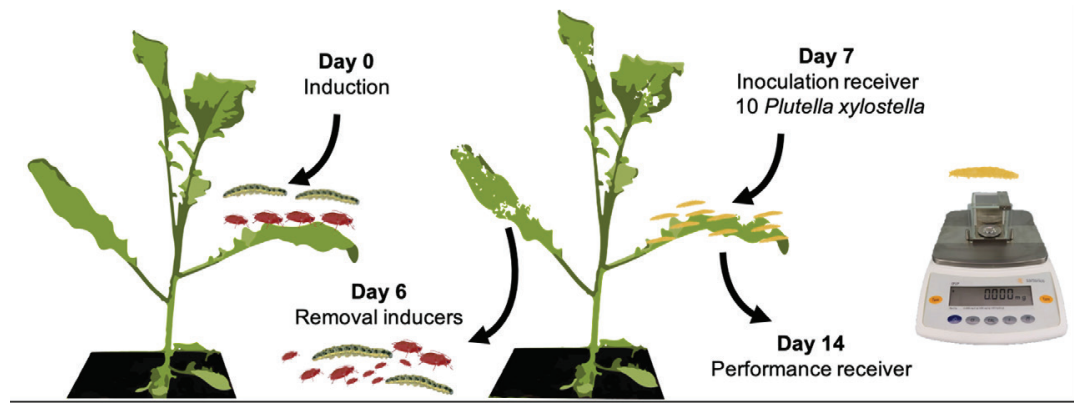

Figure 1. Experimental setup used to measure the performance of $P$. xylostella caterpillars on plants previously attacked by different species richness of phloem feeders, chewers or a mix of both (51 insect combinations). 
Table 2.

\begin{tabular}{|c|c|c|c|c|c|c|c|}
\hline \multirow[b]{2}{*}{ Group } & \multirow[b]{2}{*}{$\begin{array}{c}\text { Species } \\
\text { richness }\end{array}$} & \multirow[b]{2}{*}{ Treatment } & \multicolumn{3}{|c|}{ Performance } & \multicolumn{2}{|c|}{ Gene expression } \\
\hline & & & \begin{tabular}{|c|} 
Plant \\
replicates
\end{tabular} & Trays & $\begin{array}{c}\text { Replicates } \\
\text { group }\end{array}$ & $\begin{array}{l}\text { Biological } \\
\text { replicates }\end{array}$ & $\begin{array}{l}\text { Replicates } \\
\text { group }\end{array}$ \\
\hline Control & \begin{tabular}{|l|}
0 \\
\end{tabular} & $\mathrm{Ctrl}$ & 10 & 2 & 10 & 5 & 5 \\
\hline \multirow{11}{*}{ 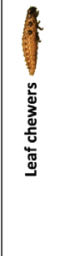 } & \multirow{4}{*}{1} & $M b$ & 10 & 2 & \multirow{4}{*}{40} & 5 & \multirow{4}{*}{20} \\
\hline & & $A r$ & 10 & 2 & & 5 & \\
\hline & & $P b$ & 10 & 2 & & 5 & \\
\hline & & $P C$ & 10 & 2 & & 5 & \\
\hline & \multirow{6}{*}{2} & $M b-A r-$ & 10 & 2 & \multirow{6}{*}{60} & 5 & \multirow{6}{*}{30} \\
\hline & & $M b-P b$ & 10 & 2 & & 5 & \\
\hline & & $M b-P c$ & 10 & 2 & & 5 & \\
\hline & & $A r-P b$ & 10 & 2 & & 5 & \\
\hline & & $A r-P C$ & 10 & 2 & & 5 & \\
\hline & & $P b-P c$ & 10 & 2 & & 5 & \\
\hline & 4 & $M b-A r-P b-P c$ & 60 & 12 & 60 & 20 & 20 \\
\hline \multirow{11}{*}{ 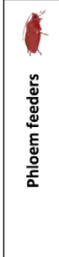 } & \multirow{4}{*}{1} & $B b$ & 10 & 2 & \multirow{4}{*}{40} & 5 & \multirow{4}{*}{20} \\
\hline & & $M p$ & 10 & 2 & & 5 & \\
\hline & & Mpn & 10 & 2 & & 5 & \\
\hline & & Le & 10 & 2 & & 5 & \\
\hline & \multirow{6}{*}{2} & $B b-M p$ & 10 & 2 & \multirow{6}{*}{60} & 5 & \\
\hline & & $B b-M p n$ & 10 & 2 & & 5 & \\
\hline & & $B b-L e$ & 10 & 2 & & 5 & \\
\hline & & Mp-Mpn & 10 & 2 & & 5 & 30 \\
\hline & & Mp-Le & 10 & 2 & & 5 & \\
\hline & & Mpn-Le & 10 & 2 & & 5 & \\
\hline & 4 & Bb-Mp-Mpn-Le & 60 & 12 & 60 & 20 & 20 \\
\hline & & $B b-M b$ & 8 & 2 & & 2 & \\
\hline & & $B b-A r$ & 8 & 2 & & 2 & \\
\hline & & $B b-P b$ & 8 & 2 & & 2 & \\
\hline & & $B b-P c$ & 8 & 2 & & 2 & \\
\hline & & $M p-M b$ & 8 & 2 & & 2 & \\
\hline & & $M p-A r$ & 8 & 2 & & 2 & \\
\hline & & $M p-P b$ & 8 & 2 & & 2 & \\
\hline & 2 & $M p-P c$ & 8 & 2 & 128 & 2 & 32 \\
\hline & 2 & $M p n-M b$ & 8 & 2 & 120 & 2 & $\sqrt{26}$ \\
\hline & & $M p n-A r$ & 8 & 2 & & 2 & \\
\hline & & $M p n-P b$ & 8 & 2 & & 2 & \\
\hline 5 & & $M p n-P c$ & 8 & 2 & & 2 & \\
\hline & & $L e-M b$ & 8 & 2 & & 2 & \\
\hline & & Le-Ar & 8 & 2 & & 2 & \\
\hline w & & $L e-P b$ & 8 & 2 & & 2 & \\
\hline हुँ & & Le-Pc & 8 & 2 & & 2 & \\
\hline$\underline{\mathrm{E}}$ & & $B b-M p-M b-A r$ & 8 & 2 & & 2 & \\
\hline$\frac{5}{0}$ & & $B b-M p-P b-P c$ & 8 & 2 & & 2 & \\
\hline$\sum_{0}^{2}$ & & $B b-M p n-M b-P c$ & 8 & 2 & & 2 & \\
\hline 4 & & $B b-M p n-A r-P b$ & 8 & 2 & & 2 & \\
\hline$\stackrel{x}{\Sigma}$ & & $B b-L e-M b-P b$ & 8 & 2 & & 2 & \\
\hline & & $B b-L e-A r-P C$ & 8 & 2 & & 2 & \\
\hline & 4 & & & & 96 & & 24 \\
\hline & & $M p-M p n-M b-P b$ & 8 & 2 & & 2 & \\
\hline & & $M p-M p n-A r-P c$ & 8 & 2 & & 2 & \\
\hline & & $M p-L e-M b-P c$ & 8 & 2 & & 2 & \\
\hline & & $M p-L e-A r-P b$ & 8 & 2 & & 2 & \\
\hline & & Mpn-Le-Mb-Ar & 8 & 2 & & 2 & \\
\hline & & $M p n-L e-P b-P c$ & 8 & 2 & & 2 & \\
\hline
\end{tabular}


day later, each plant was infested with 10 second instar P. xylostella larvae, acting as subsequent herbivore, or receiver. The mass of $P$. xylostella caterpillars was measured after six days of feeding on the control and induced plants as a proxy of plant resistance This was done by recapturing the P. xylostella larvae and weighing each individual on a Sartorius ${ }^{\circledR}$ - CP2P - Analytical Balance (accuracy $0.001 \mathrm{mg})$.

\section{Comparing direction of effects by feeding guilds}

After establishing in separate experiments for feeding guild the effect of species richness within feeding guild on the performance of P. xylostella and in which direction each feeding guild affected P. xylostella performance, we performed an additional experiment to directly compare the effect of feeding guild on $P$. xylostella performance. We selected the most extreme treatments of each main experiment: CON-0 (control), phloem-feeder (PHLO-4), leaf-chewer (CHEW-4) and mixed (MIX-4) and prepared 24 plant replicates per treatment (Table S2). We randomized individual plants over the greenhouse and followed the same protocol of inducing the plants, removing the herbivores used for induction and assessing the performance of $P$. xylostella caterpillars on these plants. All performance experiments were performed in the same greenhouse compartment (December 2017 to July 2018) (19 C, 60-70\% RH and 16:8 h L:D photo regime).

Table 2. Overview of treatments and replicates used for performance of Plutella xylostella experiment (approach 1) and for the experiment where we measured expression of defence related genes on Brassica nigra leaves. We show replicates per treatment and per species richness within each group (leaf chewer, phloem feeder, mix). P. xylostella performance was measured in three separate experiments (leaf chewer, aphid, mix), each of them divided in two blocks over time. Each of these blocks contained five control plants (species richness 0, no insects). The replicates shown are the total of replicates for the two blocks together. Each replicate consisted of one $B$. nigra plant where we inoculated ten $P$. xylostella. The gene expression analysis was done in a single experiment. Here, each biological replicate consisted of a pool of leaf disks from three plants. We prepared a set of biological replicates that were sampled $48 \mathrm{~h}$ after induction, and another set that was sampled at $96 \mathrm{~h}$ after induction. Each plant was sampled only once. Leaf chewers: $M b=$ Mamestra brassicae, $A r=$ Athalia rosae, $P b=$ Pieris brassicae, $P c=$ Phaedon cochleariae. Aphids: $B b=$ Brevicoryne brassicae, $M p=$ Myzus persicae, $M p n=$ Myzus persicae sub. nicotianae, Le= Lipaphis erysismi. 
Analysis of $\mathrm{B}$. nigra gene expression

We characterised $B$. nigra physiological responses to attack by the herbivore combinations in a single separate experiment (same treatments used for measuring performance, Table 2). The experiment was conducted in a different greenhouse compartment than the performance tests, but with similar climate conditions (July-August 2018) $\left(19^{\circ} \mathrm{C}, 60-70 \% \mathrm{RH}\right.$ and 16:8 $\mathrm{h} \mathrm{L:D}$ photo regime). To characterise plant responses, we selected homologues of genes that are known to regulate JA- and SA-plant responses to insect-herbivore attack. We analysed expression levels of the JA-biosynthesis gene LIPOXYGENASE 2 (LOX2), the JA-responsive gene VEGETATIVE STORAGE PROTEIN2 (VSP2), the SA-biosynthesis gene ISOCHORISMATE SYNTHASE (ICS), the SA-responsive gene PATHOGENESIS-RELATED PROTEIN 1 (PR1) and the crosstalk gene $W R K Y 70$. Biosynthesis of the lipid-derived phytohormone JA starts when injury of plant cells releases $\alpha$-linolenic acid from the plastid membranes (Wasternack \& Song, 2017). A key regulator of the first steps in JA biosynthesis is the enzyme lipoxygenase (LOX) which activates expression of JA-biosynthetic genes, such as LOX2 (Bell et al., 1995). The bioactive form of JA (JA-Ile) is synthetized after a few enzymatic steps and transported to the nucleus. JA-Ile triggers the degradation of the JASMONATE-ZIM DOMAIN $(J A Z)$, a repressor of JA biosynthesis. JAZ degradation releases repression of MYC transcription factors, resulting in expression of JA-responsive genes, such as VSP2 (Erb \& Reymond, 2019). SA can be synthesized via the PHENYLALANINE AMMONLA LYASE (PAL) and the ISOCHORISMATE SYNTHASE (ICS) enzymatic pathways (Pieterse et al., 2012). Signalling downstream of SA biosynthesis is regulated by transcription factors that activate expression of pathogenesis-related defence genes (PR genes) (Pieterse et al., 2012). Additionally, WRKY transcription factors play a key role in SA signalling, as they activate or repress $\mathrm{SA}$ responses.

We focussed on characterising how plants respond to attack when herbivores are coming from the same feeding guild (phloem-feeders or leafchewers) or from multiple feeding guilds. Within feeding guild, we then 
addressed whether individual herbivores, number of herbivore species and specific combinations differ in induced plant responses. Gene expression in response to herbivory was assessed at 48 and 96 hours after induction, using a different set of plants per time point so that plants were only sampled once. Our earlier experiments with $B$. nigra identified that with the two time points the expression patterns of the selected genes can be well characterised (Mertens et al., unpublished). We prepared five biological replicates per treatment for control (CON-0), leaf-chewer (CHEW-1; for each of the four leaf-chewer species) and phloem-feeder (PHLO-1; for each of the four phloem-feeder species) and five replicates for each CHEW-2 and PHLO-2 species combination (six species combinations for leaf chewers and phloem feeders) (Table 2). We prepared 20 biological replicates for treatments with four herbivore species CHEW-4 and PHLO-4 to balance the number of replicates across species richness treatments. MIX-2 (16 species combinations) and MIX-4 (12 species combinations) had two biological replicates per species combination, which resulted in 32 biological replicates for MIX-2 and 24 for MIX-4, respectively. Each biological replicate contained a leaf disc $(\varnothing=2 \mathrm{~cm})$ that was taken with a sterilized puncher from three plants with the same treatment, sampling each plant only once. Leaf samples were placed in an Eppendorf tube, immediately flash frozen in liquid nitrogen and stored at $-80{ }^{\circ} \mathrm{C}$ until further analysis (Fig. 1). Induction of plants with the herbivore treatments was performed on the youngest fully-expanded leaf. Directly after induction, the induced leaf was enclosed in a mesh bag to confine herbivores to a single leaf. Control plants received a mesh bag without insects. Biological replicates represented by the group of three plants that would be combined in a single sample were randomly placed in the greenhouse.

\section{RNA extraction and PCR protocol}

The frozen samples were ground to a fine powder with a sterile pestle. This was followed by RNA extraction using the RNeasy 96 Kit (Qiagen) with an adjusted protocol for plants. $450 \mu \mathrm{L}$ of RTL buffer were added to each Eppendorf tube (which contained approximately $50 \mathrm{mg}$ of frozen, ground leaf sample). Samples were incubated at $56{ }^{\circ} \mathrm{C}$ for $1-3$ minutes and were centrifuged 
at 10,000 Relative Centrifugal Force (RCF), until a tight pellet was formed (8-10 minutes). The supernatant was transferred to new Eppendorf tubes and mixed with 0.5 volumes of $96 \%$ ethanol. Each sample was transferred to a 96-RNeasy plate and that was centrifuged 6 minutes at 3700 RCF. The flow-through was discarded, and each well was treated with $80 \mu \mathrm{L}$ of a solution of DNase I in RDN buffer (1:7) which was added to the tube directly onto each membrane and was incubated for 15 minutes at room temperature. To each sample, 800 $\mu \mathrm{L}$ of RWI buffer were added, and after 5 minutes of incubation the plate was centrifuged for 6 minutes at $3700 \mathrm{G}$ and the flow-through was discarded. Now, $800 \mu \mathrm{L}$ of RPE buffer were added to each sample, and the plate was centrifuged for 6 minutes at $3700 \mathrm{RCF}$, the flow-through was discarded and the plate was centrifuged for 10 minutes at 3700 RCF. The plate was placed on a rack of collection microtubes, where $45 \mu \mathrm{L}$ of RNAse free water (Qiagen) were added to each sample and after one-minute incubation, the plate was centrifuged for 6 minutes at 3700 RCF. The RNA concentration was measured using a DS-II FX Spectrophotometer/Fluometer (DeNoVix). Samples were diluted and adjusted to an RNA concentration of $66.6 \mathrm{ng} / \mu \mathrm{l}$. From the RNA samples cDNA was synthesized, using the SensiFAST cDNA synthesis kit (Bioline). We quantified the expression levels of the target genes LOX2, VSP2, ICS and PR1 and of the reference genes $G A P D H$ and $S A R 1 A$ by qPCR using the SensiFAST SYBR noROX kit (Bioline). We added 5 ng of the cDNA template to the reaction with a total volume of $20 \mu \mathrm{L}$. The reactions were performed using a CFX96 Touch ${ }^{\text {TM }}$ Real-Time PCR Detection System (Biorad). All reactions were conducted using two technical replicates and samples were omitted from further analyses if the difference in expression of technical replicates was higher than 0.5 (4\% of the samples). Plate setups included negative controls (no template) and inter-run calibrators.

We tested the following reference genes: ACTIN-2 (ACT2), BETATUBULINE (B-TUB), ELONGATION FACTOR-1 (EF1), PEROXIDASE 4 (PER4), SECRETION ASSOCLATED RAS RELATED GTPASE 1 A (SAR1A), GLYCERALDEHYDE-3-PHOSPHATE DEHYDROGENASE 
$(G A P D H)$. The last two reference genes were selected because they had highest expression stability regardless of the treatment (primer sequences are presented in Table S3). We used the expression of the reference genes to calculate the relative expression of genes of interest in each sample subjected to the different treatments.

\section{Statistical analysis}

\section{Performance data}

We first analysed for each of the feeding guilds separately how species richness of inducing herbivores affected plant resistance to the subsequent feeding by P. xylostella caterpillars. The performance data (weight of individual $P$. xylostella caterpillars) was cube-root transformed (third level root transformation) to better approach the assumptions of normality and constant variance. We fitted Mixed Linear Models (MLM), using a fixed effect for species richness with the levels of richness 1, 2 and 4 for the model on phloem feeders and the model for leaf-chewers, the levels 2, 4 for the model of the mixed feeding guilds. We included the sub-treatments of each of the unique species or species combinations (11 for phloem feeders, 11 for leaf chewers, and 28 for mix). We present the outcomes of these models with two sets of random effects (Table S4). One set of models that included the random structure for blocks, trays within blocks, plants within trays, and residual error. The second set of models excluded the tray effect from the random structure. Even though in this particular statistical approach the random factor "tray" unexpectedly explained a substantial amount of variation, we purposely removed this factor from the model. The tray corresponded with the variation of sub-treatments of species combinations within the richness levels and likely at least to an extend contains the biological variation caused by unique species combinations. We kept a smaller scale random factor of plant, and we aimed to test the biological effect of the sub-treatments within each main experiment (species included in phloem-feeder, leaf-chewer and mixed treatments) represented by trays with sub-treatments randomly distributed in the greenhouse. Based upon the fitted model, by averaging over the insect combinations that constituted a richness level 
within guild, and combining the estimated means linearly, we estimated linear regression lines for species richness within guild, using facilities for user-defined linear combinations of the software. Next, we tested nullity of slopes within guilds to identify whether there was a significant positive or negative correlation between species richness of inducers and performance of P. xylostella (slopes excluded performance of caterpillars on undamaged plants). Besides linear trends, also quadratic trends were tested. Since both analyses gave similar results, we present only linear trends in the results section. With the Mixed Model, we then tested for significance of the different richness levels with performance of the caterpillars on undamaged control plants. These analyses were followed by post-hoc analyses to compare individual sub-treatments. Finally, we compared the slopes for the three feeding guilds obtained in the separate experiments among each other to identify whether relationships between species richness and P. xylostella performance differed for the feeding guilds. Because this was an indirect comparison combining data obtained at different moments, we had performed an additional experiment that directly compared performance of P. xylostella on undamaged control plants, and the most extreme richness of phloem-feeders, leaf-chewers or a mix of feeding guilds. Here individual plants were placed randomly distributed in the greenhouse and a single block was performed. These data were analysed with a Mixed Model for the fixed treatment effect feeding guild (CON-0, PHLO-4, CHEW-4, MIX-4). For the random part, besides the residual variance, only random effects for plants were included.

Statistical analysis for herbivore performance was done in $\mathrm{R}$ version 3.6.1 (R Core Team, 2019) and using PROC MIXED of SAS (version 6.4). For all hypothesis tests we used 0.05 as level of significance.

\section{Gene expression}

Gene-expression data was imported to qBase+ 3.1 (Biogazelle) to calculate the relative gene expression level between different samples for a given target gene (LOX2, VSP2, ICS, PR1, WRKY70) corrected by the expression 
value of the reference genes $(G A P D H, S A R 1 A)$. Data was corrected for differences between runs using inter-run calibrators and data was Log10 transformed to meet the assumptions of the model. For each of the five genes and separately per time point, we ran General Linear Models (GLM) with PostHoc test (LSD) in R version 3.6.1 (R Core Team, 2019). We first tested whether the nine different treatment groups based on the combination of feeding guild and species richness differed in expression levels. These analyses identified that expression patterns were strongly determined by feeding guild. We then deepened the analysis by testing in separate models per feeding guild whether the species richness levels significantly affected gene expression, and used posthoc analyses to compare individual richness levels within feeding guild as well as individual sub-treatments represented by the unique herbivore combinations within feeding guild.

\section{RESULTS}

\section{Species richness affects induced plantresistance}

Plants attacked by a higher species richness of phloem feeders became more susceptible to caterpillars of the subsequent attacker P. xylostella as indicated by an increased caterpillar performance on plants induced by larger number of aphid species (Significant positive slope of species richness 1-24, with tray: $\mathrm{P}=0.095$; without tray $\mathrm{P}<0.01$; Fig. $2 \mathrm{a}$ ). A richness of four aphid species resulted in significant susceptibility to $P$. xylostella compared to its performance on undamaged control plants and susceptibility increased when four species induced plants compared to one or two aphid species (Mixed Model; without tray $\mathrm{F}_{3,438}=4.66 ; \mathrm{P}=0.0032$ ) (Fig 3a). Individual attack by each of the four phloem-feeding species only slightly enhanced performance of $P$. xylostella to levels not significantly different from performance on undamaged plants (Fig. 3b). However, the specific combination of initial attack by the aphid species $M$. persicae sub. nicotianae $-M$. persicae, $M$. persicae sub. nicotianae $-L$. erysismi or L. erysismi - B. brassicae significantly enhanced performance of P. xylostella caterpillars (Fig. 3b). 
(a)

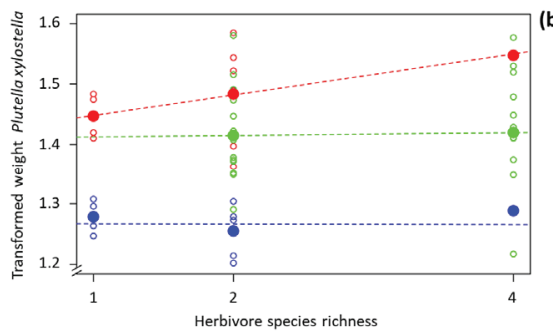

(b)

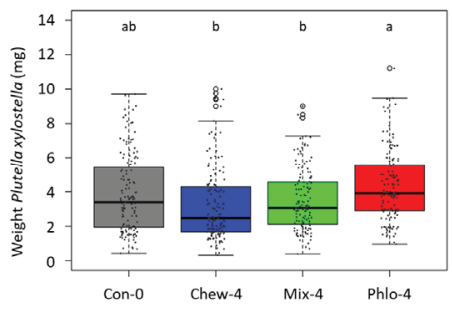

Figure 2. (a) Regression line of the ttransformed performance of Plutella xylostella on Brassica nigra plants. Performance of caterpillars in different experimental blocks is presented in weight corrected to the average weight of $P$. xylostella on untreated control plants across all blocks. Plants were treated with an increasing richness (1, 2 or 4 species) of phloem feeders (red), leaf chewers (blue), or a mix of both (green), or were left untreated. The open circles represent averages within each sub-treatment (i.e. insect combination). The phloem-feeder and leaf-chewer experiments contained four sub-treatments for species richness 1, six sub-treatments for species richness 2 and one sub-treatment for species richness 4 . The mixed species richness experiment contained sixteen sub-treatments for mixed species richness 2 and twelve sub-treatments for mixed species richness 4 .. The closed circles represent averages of the sub-treatments within each species richness level. Lighter-coloured circles show treatments that only received 1 herbivore species, middle-dark-coloured circles show treatments that received 2 herbivore species, darker-coloured circles show treatments that received 4 herbivore species. (b) Performance of $P$. xylostella on approach 2: In one experiment, plants were treated with four species of phloem feeders (Phlo-4), leaf chewers (Chew-4), a mix of both (Mix-4) or left untreated (Con-0) $(\mathrm{N}=24)$. Boxplot height corresponds to the first and third quartiles (Q1 and Q3), and the middle line to the median. Letters above the boxplots show significant differences (MLM, posthoc Tukey). Letters for panel c can be found in Table S4. Leaf chewers: Mamestra brassicae (Mb), Phaedon cochleariae (Pc), Pieris brassicae (Pb), Athalia rosae (Ar). Phloem-feeding aphids Myzus persicae sub. nicotianae (Mpn), Lipaphis erysimi (Le), Myzus persicae (Mp), Brevicoryne brassicae (Bb).

In contrast, a higher species richness of leaf-chewers did not lead to effects on resistance to P. xylostella (No significant slope of species richness 1-24; with tray $\mathrm{P}=0.97$; without tray $\mathrm{P}=0.97$; Fig. 2a). Plant induced responses to feeding by one, two or four species of leaf-chewers equally enhanced resistance to $P$. xylostella caterpillars as indicated by decreased performance of these caterpillars compared to their performance on undamaged control plants (Mixed Model; without tray $\mathrm{F}_{3,248}=5.39 ; \mathrm{P}=0.013$ ) (Fig 3b). All four leafchewing herbivores reduced $P$. xylostella performance similarly when attacking the plant as individual species (Fig. 3a). The combination of the leaf chewers 
A. rosae and $P$. cocbleariae had the strongest negative effect on the performance of P. xylostella caterpillars, but did not differ significantly from other treatments with two leaf-chewer species (CHEW-2) or plants damaged by single chewer species (CHEW-1) or the full set of four species (CHEW-4) (Fig. 3a). Induction of plants by a mix of phloem-feeding and leaf-chewing herbivores with either two or four different species did not affect the performance of P. xylostella caterpillars (Slope for richness 2 and 4 not different from 0: with tray $\mathrm{P}=$ 0.81 ; without tray $\mathrm{P}=0.81$ ) (Fig. $2 \mathrm{a}$ ) and on average the performance of $P$. xylostella on plants induced with a mix of feeding guilds was not different from its performance on undamaged control plants (Mixed Model; without tray $\mathrm{F}_{2,485}$ $=0.03 ; \mathrm{P}=0.97$ ) (Fig 3c). Despite these overall neutral effects, plants previously attacked by a specific combination of $M$. persicae sub. nicotianae $-A$. rosae or a mix of the four species $M$. persicae sub. nicotianae - L. erysimi $-M$. brassicae $-P$. cocbleariae became less resistant to larvae of P. xylostella (Table S5, Fig. 3c). In contrast, plants previously attacked by a mix of the four species B. brassicae $-M$. persicae - P. brassicae - A. rosae became more resistant to larvae of P. xylostella (Table S5, Fig. 3c).

In our second independent experiment where individual plants could be randomly distributed in the greenhouse, we confirmed that performance of P. xylostella caterpillars differed for plants induced by phloem-feeders, leafchewers or a mix of herbivores from different feeding guilds (Mixed Model, $\mathrm{F}_{3,77}=4.37, \mathrm{P}=0.0068$ ) (Fig 2b). Direct comparison of P. xylostella performance on plants induced by species richness of four herbivores supported that phloem-feeders increased performance of $P$. xylostella caterpillars and differed from the reduced performance of caterpillars feeding on leaf-chewer induced plants (Fig 2b). Caterpillars feeding from plants induced by a mix of feeding guilds had intermediate performance and had significantly lower average mass compared to caterpillars feeding on phloem-feeding induced plants (Fig 2b). Indirect comparison of the direction of slopes in the first series of experiments further supports that the direction of effect of species richness differs between phloem-feeders and the slope of leaf-chewers or the mixed 


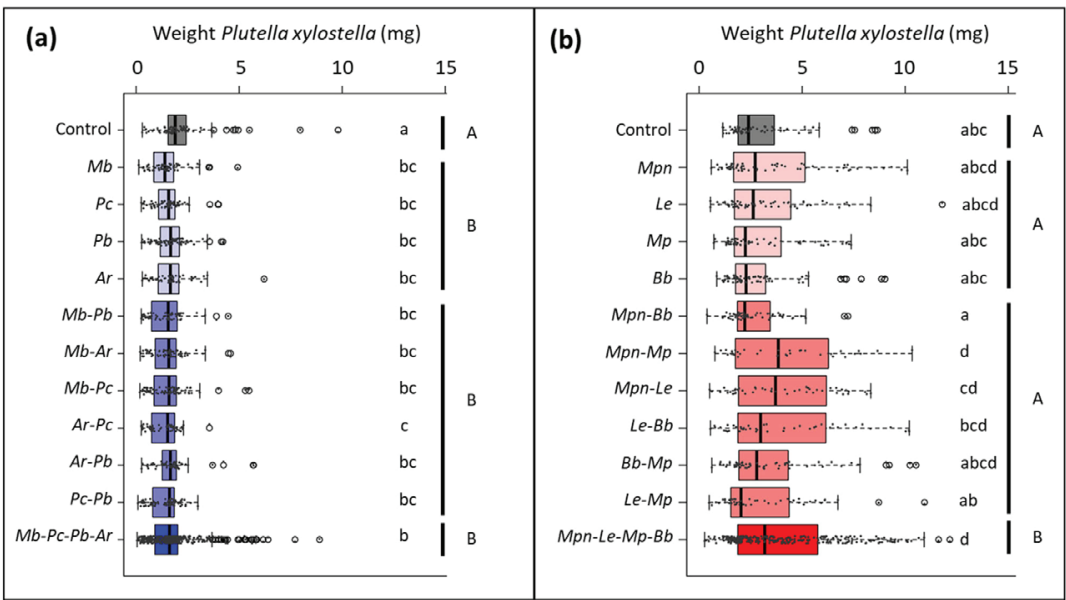

(c)

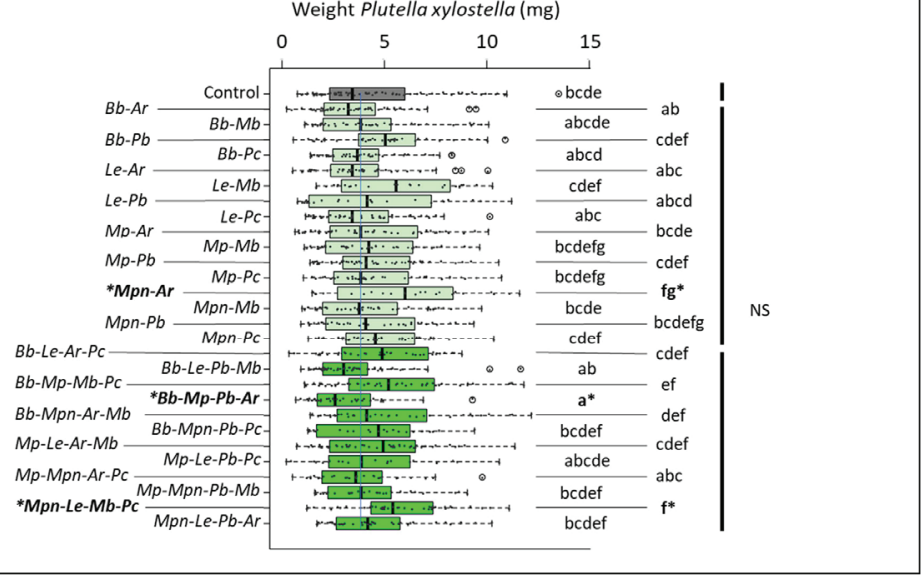

Figure 3. Weight (mg) of Plutella xylostella larvae after feeding for six days from Brassica nigra plants previously attacked by leaf chewers (blue), phloem feeders (red), a mix of both (green), or from untreated plants (grey, control). Panels (a), (b) and (c) show the performance of $P$. xylostella in approach 1 , where we measured performance in three separate experiments. Lighter boxplots show treatments that only received 1 herbivore species, middle-dark boxplots show treatments that received 2 herbivore species, darker boxplots show treatments that received 4 herbivore species. (a) Performance of $P$. xylostella on plants previously attacked by leaf chewers $(\mathrm{N}=10)$. (b) Performance of $P$. xylostella on plants previously attacked by phloem feeders $(\mathrm{N}=10)$ (c) Performance of P. xylostella on plants previously attacked by a mix of leaf chewers and phloem feeders 
( $\mathrm{N}=10$ for control, $\mathrm{N}=8$ for the rest). Combination of inducers that affected $P$. xylostella growth (compared to control, untreated plants) are marked in bold and with an asterisk. Boxplot height corresponds to the first and third quartiles (Q1 and Q3), and the middle line to the median. Letters above the boxplots show significant differences (MLM, posthoc Tukey). Letters for panel c can be found in Table S4. NS= not significant Leaf chewers: Mamestra brassicae (Mb), Phaedon cochleariae (Pc), Pieris brassicae (Pb), Athalia rosae (Ar). Phloem-feeding aphids Myzus persicae sub. nicotianae (Mpn), Lipaphis erysimi (Le), Myzus persicae (Mp), Brevicoryne brassicae (Bb).

herbivore treatments (PHLO versus $\mathrm{CHEW}$ with tray $\mathrm{P}=0.22$, without tray $\mathrm{P}$ $<0.05$; PHLO versus MIX with tray $\mathrm{P}=0.24$, without tray $\mathrm{P}<0.05$; CHEW versus MIX with tray $\mathrm{P}=0.86$, without tray $\mathrm{P}=0.84)$.

To summarize, species richness of initial attackers as well as species composition across feeding guilds affects induced plant resistance to subsequent herbivory. Plants attacked by phloem-feeding insects became more vulnerable to larvae of P. xylostella, whereas plants attacked by leaf chewers became more resistant to $P$. xylostella caterpillars. Species richness of phloem feeders was more important in affecting $P$. xylostella performance than richness of leaf chewers. For leaf-chewers the presence of a single herbivore species caused similar effects compared to four species of leaf-chewing herbivores. Plutella xylostella caterpillars performed equally well on plants induced with a mix of herbivore species from the two feeding guilds regardless of species richness and on plants that had not been exposed to herbivores.

\section{Feeding guild specific induced gene expression}

Brassica nigra attacked during 48 and $96 \mathrm{~h}$ by leaf chewers, or by a mix of leaf chewers and phloem feeders showed induced expression of the JAbiosynthesis and JA-responsive genes (LOX2 and VSP2, respectively) and of the SA-responsive gene (PR1) (LM 48h: LOX2 $\mathrm{F}_{8,149}=20.58, \mathrm{P}<0.001$; VSP2 $\mathrm{F}_{8,149}=24.93, \mathrm{P}<0.001 ;$ PR1 $\mathrm{F}_{8,149}=4.64, \mathrm{P}<0.001$, Fig. 4; LM 96h: LOX2 $\mathrm{F}_{8,149}=43.70, \mathrm{P}<0.001 ;$ VSP2 $\mathrm{F}_{8,149}=18.0, \mathrm{P}<0.001 ;$ PR1 $\mathrm{F}_{8,149}=3.51$, $\mathrm{P}<0.001$, Fig. S1). In contrast, phloem feeders did not induce significant expression of these genes compared to undamaged control plants. None of the herbivore treatments significantly affected expression of the SA- 
biosynthesis gene ICS and of the crosstalk gene WRKY70 at either 48 or $96 \mathrm{~h}$ of herbivore attack (LM, 48h: ICS $\mathrm{F}_{8,149}=1.0, \mathrm{P}=0.44 ; W R K Y 70 \mathrm{~F}_{8,149}$ $=1.35 \mathrm{P}=0.22$, Fig. 4; LM 96h: ICS $\mathrm{F}_{8,149}=1.08, \mathrm{P}=0.38 ; W^{2} K Y 70 \mathrm{~F}_{8,149}$ $=1.12 \mathrm{P}=0.30$, Fig. S1). 48 and $96 \mathrm{~h}$ of feeding by the leaf chewers and by each of the leaf-chewer species combinations induced the JA-biosynthesis gene LOX2 (Fig. S2 \& S3). In addition to the direction of plant responses driven by feeding guild, within feeding guilds species richness affected gene expression only for leaf-chewing herbivores (Figs. S2-S5). For leaf-chewing herbivores, 96h of feeding by a combination of two herbivores enhanced gene expression of LOX2 (F = 12.16, P < 0.001) and VSP2 $(\mathrm{F}=4.54, \mathrm{P}<$ 0.01) compared to single herbivore attack (Fig. S3). Herbivory by four species could not be discriminated in gene expression patterns from those induced by one or two leaf-chewing herbivores (Fig. S3). Individual leaf-chewer species and combinations of specific leaf chewers differed in the magnitude of induced expression of LOX2 (Fig. S3a). Plants attacked by P. cochleariae or by $A$. rosae had stronger $L O X 2$ induction than plants attacked by $M$. brassicae. Feeding by the combinations of $A$. rosae $-P$. cochleariae, $A$. rosae $-P$. brassicae or P. brassicae - P. cocbleariae induced the highest expression levels of LOX2 (Fig. S3a). Similarly, most of the leaf chewers (combinations) induced the JA-responsive gene VSP2 with the only exception of plants attacked by $M$. brassicae, in which expression of VSP2 was not different from control plants. The combination of P. brassicae - P. cocbleariae caused the strongest induction of VSP2 at $96 \mathrm{~h}$ (Fig. S3b). The SA-responsive gene PR1 was induced by attack of some (combination of) leaf chewers (Fig. S3d). Single attack by $P$. cocbleariae or $A$. rosae, dual attack by $M$. brassicae - A. rosae, $M$. brassicae $-P$. cocbleariae, or A. rosae $-P$. cochleariae and feeding by the four leaf-chewers induced expression of PR1 at 96h (Fig. S4d). In contrast, feeding by phloem feeders and by each of the phloem-feeder species combinations did not induce any of the defence marker genes tested at 48 or $96 \mathrm{~h}$ after attack (Fig. S4 \& S5), except for Myzus persicae that induced LOX2 expression $48 \mathrm{~h}$ after feeding (Fig. S4). 
In summary, feeding by the leaf chewers individually or in combination, induced the expression of the JA-biosynthesis and JA responsive genes LOX2 and VSP2, respectively. Feeding by a mix of leaf-chewing and phloem-feeding insects, also induced LOX2 and VSP2, but to a lesser extent. Feeding by the phloem feeders, or by combination of phloem feeders did not induce any of the marker genes tested but repressed the expression of the JA-responsive gene VSP2 48 h after attack.

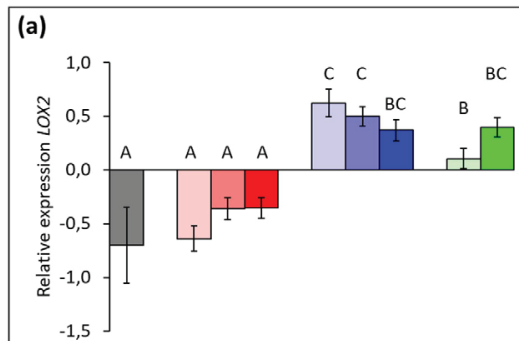

(b)

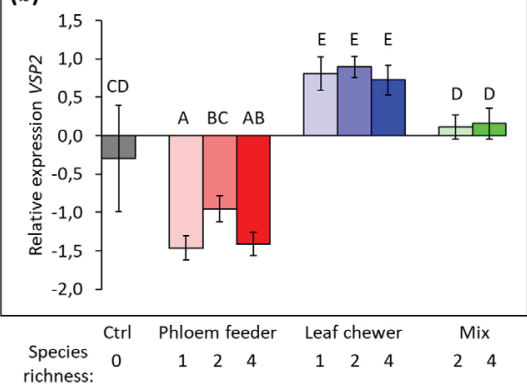

\section{(c)}

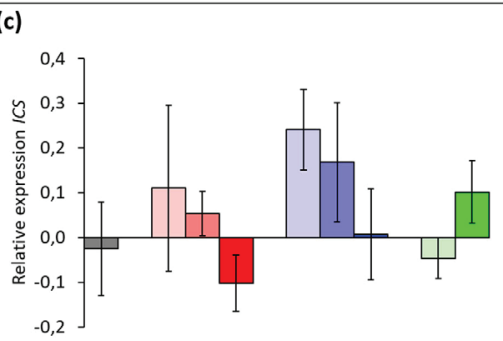

(d)

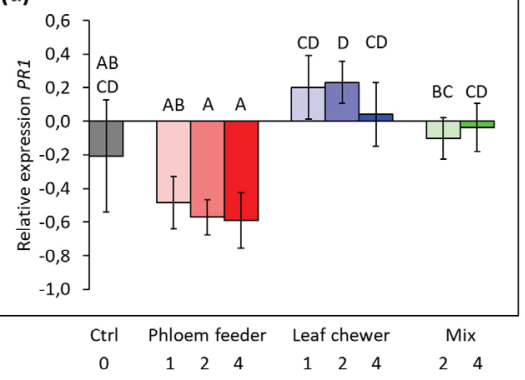

Figure 4. Relative gene expression of Brassica nigra leaves at $48 \mathrm{~h}$ after infesting them with an increasing species richness (1, 2 or 4 species) of aphids, chewers, a mix of both or untreated plants (Ctrl). We measured the expression of JA-biosynthesis and JA-responsive genes LOX2, and VSP2 (a) and (b), and the SA-biosynthesis and SA-responsive- genes ICS and PR1 (c) and (d). Bars represent mean \pm SE of log transformed data. Gene expression is relative to the expression level of two reference genes GAPDH and SAR1A. Bars not sharing letters are significantly different from each other (LM, post hoc LSD. 


\section{DISCUSSION}

Multi-herbivore attack, species richness of herbivore attack as well as the composition of the species across feeding guilds influenced induced plant responses and resistance to subsequent herbivory. Plant responses to phloemfeeding insects promoted the growth of P. xylostella, whereas plant responses to leaf chewers reduced the performance of P. xylostella caterpillars. The higher the species richness of phloem feeders attacking the plant, the more vulnerable the plant became to larvae of the subsequent attacker P. xylostella. In contrast, induction by any combination of leaf-chewers regardless of species richness led to stronger resistance to P. xylostella. When the plant was attacked by a mix of phloem feeders and leaf chewers $P$. xylostella caterpillars performed equally well on plants induced with a mix of herbivore species from the two feeding guilds and on plants that had not received initial herbivore attack. Unexpectedly, in our study, the factor tray explained a substantial amount of variation. Plants sharing the same tray, were not only receiving the same herbivore, but could have affected each-other more strongly than plants in neighbouring trays through volatile or root communication via the shared pool of water. The compromise of plants in trays we had to make for feasibility of the experiment confounded biological effects associated with trays and those on our treatment level. Because trays were randomly distributed in the greenhouse having different neighbouring treatments, we deem it unlikely that volatile or root communication drives the patterns observed in our experiments. By using multiple species combinations per species richness level that were randomly placed in the greenhouse, independent blocks and experiments that all present similar findings, the most likely conclusion is the biological effect of species richness and feeding guild of the herbivores driving plant responses and resistance to P. xylostella.

The stronger susceptibility to larvae of P. xylostella after induction by a richer phloem feeder community suggests an additive effect of each phloem feeder species on suppressing plant responses to a leaf chewer. When feeding from the phloem sap, aphids inject effectors (i.e. aphid salivary proteins) that alter the plant primary and secondary metabolism (Mutti et al., 2008; Giordanengo 
et al., 2010; Elzinga \& Jander, 2013; Züst \& Agrawal, 2016; Ahman et al., 2019; Jiang et al., 2019). Excretion of salivary effectors by $M$. persicae has been found to reduce plant secondary metabolite production and callose deposition, benefitting aphid population growth (Elzinga et al., 2014). The enhanced performance of $P$. xylostella on plants previously attacked by a higher species richness of phloem feeders, could potentially be attributed to a higher diversity of salivary effectors with more aphid species increasing the strength of the antagonistic effect of SA regulated resistance on JA responses. In our study, aphid feeding suppressed the expression of the JA-responsive gene VSP2 48h after attack. The JA pathway is a well-known defence pathway against insect herbivores and thus JA suppression is a potential explanation of the increased growth of P. xylostella on aphid-induced plants (Zarate et al., 2007; Onkokesung et al., 2010). The JA suppression, might be attributed to the aphids manipulating the host plant (Will et al., 2007; Ahman et al., 2019). However, we did not detect an aphid-induced expression of any of the two SA-marker genes studied nor the SA/JA crosstalk gene $W R K Y 70$. Thus, we do not have support to explain the aphid-induced changes based on the SA/JA negative crosstalk paradigm (Kroes et al., 2015; Onkokesung et al., 2016). Further research is needed to unravel the mechanisms behind the aphid-induced facilitation of the growth of P. xylostella.

In contrast with the phloem-feeder-induced vulnerability to P. xylostella, the growth of $P$. xylostella larvae was negatively affected by previous chewer attack with no role for species richness. Attack by a single leaf-chewer had a similar reduction on P. xylostella performance compared with treatments that were richer in leaf-chewer species. The absence of effect caused by species richness of chewers compared to the effect of richness caused by phloem feeders contrasts with the higher taxonomic richness used in the chewers than in the phloem feeders (all aphids). Thus, even with a wider phylogenetic richness of chewer species, combining these species did not strongly affect plant responses to chewing herbivores. Also in research on lima bean (Phaseolus lunatus), induced plant responses were not herbivore-specific and simultaneous attack by two chewers had a similar effect compared to single attack (Moreira et al., 2015). On 
wild radish plants (Raphanus raphanistrum and R. sativus) previously attacked by P. xylostella or $S$. exigua became more resistant to larvae of S. exigua, Trichoplusia $n i$ (cabbage looper) and Pieris rapae (small cabbage butterfly) (Agrawal, 1999; Agrawal, 2000; Williams et al., 2005; Rodriguez-Saona et al., 2010). Interestingly in the study on wild radish, the strength and direction of the plant-mediated effect of chewer on chewer depended on the species involved, as previous infestation by $P$. rapae induced resistance only to $S$. exigua and $P$. rapae, and previous damage by $T$. $n i$ (generalist) did not affect the performance of any of the herbivores (Agrawal, 2000). The fact that all leaf chewers and their combinations in our study equally affected plant resistance to a subsequent attacker only partly matches our molecular analysis, where we found that all chewers (and all chewer combinations) induce the JA-biosynthesis and JA-responsive genes LOX2 and VSP2 at the two time-points measured. However, single leaf chewer species induced these genes less strongly than a combination of two leaf chewer species. In $A$. thaliana, leaf chewers had a strong induction of the JA-responsive gene VSP2, and also became more resistant to subsequent chewers (Rasmann et al., 2015). Additionally, we found that feeding by chewers induced expression of the SA-responsive gene PR1. Similarly, other studies reported induction of both SA and JA pathways upon leaf chewer feeding (Heidel \& Baldwin, 2004; Li et al., 2016a, b; Arena et al., 2018; Kielkiewicz et al., 2019). In Brassica interactions with chewing insects, specificty in response to leaf chewing herbivores may be low and suggests a less important role of Herbivore Associated Molecular Patterns (HAMP) and herbivore specific effectors than found for other plant-insect interactions (Erb \& Reymond, 2019).

Plants simultaneously attacked by leaf chewers plus phloem feeders show similar resistance to larvae of $P$. xylostella compared to plants that did not receive herbivory. This suggests that the effect of aphid-induced vulnerability cancels out the effect of chewer-induced resistance to larvae of P. xylostella. In line with our findings, cotton plants simultaneously attacked by the chewer $S$. exigua and by the phloem feeder Bemisia tabaci emitted lower amounts of herbivore-induced plant volatiles than plants that were only attacked by S. exigua (Rodriguez- 
Saona et al., 2003). Likewise, S. exigua oviposition was deterred on tomato plants damaged by chewers, but the deterrence disappeared on plants that were simultaneously damaged by aphids and chewers (Rodriguez-Saona et al., 2005). In our study, the expression of the two JA-related genes investigated was lower on plants simultaneously attacked by aphids and chewers compared to plants only under chewer attack. Similarly, caterpillar feeding on milkweed (Asclepias syriaca) induced JA, cardenolides and latex, and this induction was substantially attenuated in the presence of aphids (Ali \& Agrawal, 2014). Likewise, in potato plants (Solanum tuberosum) feeding by $M$. persicae induced solanine levels (potato secondary metabolite) and the induction disappeared upon feeding by the chewer Leptinotarsa decemlineata (Davidson-Lowe et al., 2019). The effect of mixed feeding guild attacks may be different across plant species. For example, tomato plants simultaneously attacked by aphids and chewers showed a similar defence response compared to plants only damaged by chewers (RodriguezSaona et al., 2005).

Our study revealed that species richness and trait composition of the attackers as a component of multi-herbivore attack may affect plant induced responses and resistance to subsequent herbivores. We show canalization of plant defences in which plant responses to an increasing species richness in phloem feeders induced vulnerability to larvae of $P$. xylostella. The canalization response suggests that initial attack determines the plant phenotype and reduces potential to convert an induced phenotype in a direction that maximizes resistance to subsequent attack (Agrawal, 2001; Viswanathan et al., 2007; Poelman et al., 2008; Utsumi et al., 2010; Stam et al., 2014). However, in cases of facing simultaneous attack by herbivores from different feeding guilds, $B$. nigra plants integrate responses to both feeding guilds, showing an intermediate phenotype. In our study, integration of plant responses to phloem feeders and leaf chewers cancels out the effect of chewer-induced resistance to larvae of P. xylostella to levels of resistance of plants that were not previously attacked by herbivores. Although our current study only scratches the surface of plant resistance strategies to multi-herbivore attack by revealing a role for species richness, it should be a 
starting point to further explore the importance of herbivore traits in multiherbivore attack such as their level of food plant specialisation, order and timing of arrival. Disentangling the role of individual species in the context of natural communities from community processes driven by biodiversity components of multi-species interactions per se may provide deeper understanding of how plant defences evolve under community complexity. Critical steps that should be taken are to evaluate whether plant plasticity is adaptive to specific and perhaps predictable sequences of attack or whether plants can rely on less specified plastic responses to retain fitness. This not only requires to move to challenging plants with complex orders of attackers in controlled greenhouse studies, but to match these patterns with their natural context and assess fitness outcomes in these interactions. Plants may be selected for induced responses that match the patterns of multi-herbivore attack in which the response to one herbivore should not compromise resistance to likely future attack (Mertens et al., 2021). We need to identify the repertoire of defence strategies that plants may use in different ecological settings to understand evolution of plastic defence strategies. Our study also identifies how challenging it is to compromise experimental design to unravel these plant strategies, which should nonetheless be taken to make it feasible to explore the scope of plant plasticity to multi-herbivore attack.

ACKNOWLEDGEMENTS The authors want to thank the Unifarm team, Sean Geurts and André Maassen for growing the plants; Pieter Rouweler, André Gidding, Frans van Aggelen and Quint Rusman for rearing some of the insect species used for the experiments; Rieta Gols, Roel van Wiechen, Lucille Chrétien, Onne Insfordink, Thibault Costaz, Angelos Mouratidis, Milena Chinchilla, Tim Möhlmann, Kay Moisan, Sandeep Sarde, Alexander Villela, Julia Friman, Peter Karssemeijer and Giuditta Beretta for their help during induction and sampling at the gene expression experiment. We thank four anonymous reviewers for constructive feedback on previous versions of this manuscript. This research was funded by the European Research Council (ERC) under the European Union's Horizon 2020 research and innovation programme (grant agreement $n^{\circ}$ 677139) to EHP. 
AUTHOR CONTRIBUTIONS M.F.B., M.D. and E.H.P. planned and designed the study. M.F.B. collected most data. M.E.B. collected data on insect performance for approach 1 for the performance of P. xylostella on plants induced by leaf-chewer and mixed herbivores and together with M.F.B collected data for the gene expression experiment. S.N.K. collected data on insect performance for approach 1 for the performance of P. xylostella on plants induced by phloem feeders. J.B. designed the primers and assisted during the gene expression experiment. G.G. carried out the statistical analyses. M.F.B., M.D. and E.H.P wrote the manuscript. 


\section{SUPPORTING INFORMATION}

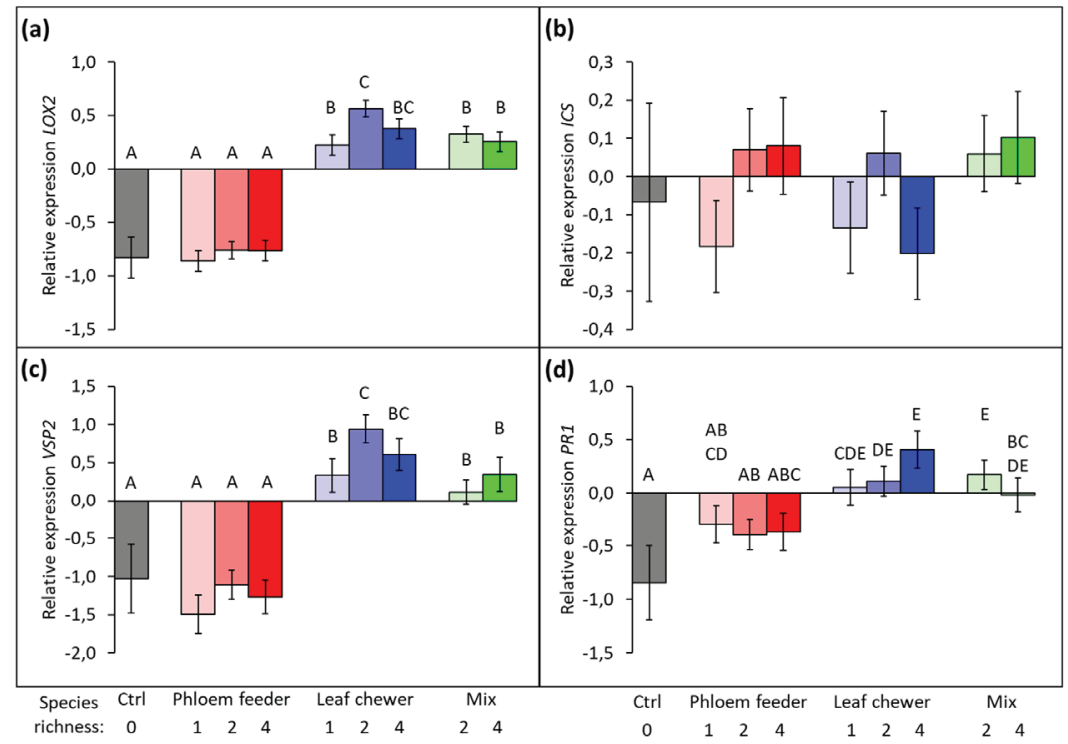

Fig. S1. Relative gene expression of Brassica nigra leaves at $96 \mathrm{~h}$ after infesting them with an increasing species richness (1, 2 or 4 species) of aphids, chewers, a mix of both or untreated plants (Ctrl). We measured the expression of JA-biosynthesis and JA-responsive genes LOX2, and VSP2 (a) and (b), and the SA-biosynthesis and SA-responsive genes ICS and PR1 (c) and (d). Bars represent mean \pm SE of log transformed data. Gene expression is relative to the expression level of two reference genes GAPDH and SAR1A. Bars not sharing letters are significantly different from each other (LM, post hoc LSD). 
Gene expression after $48 \mathrm{~h}$ of leaf chewer attack

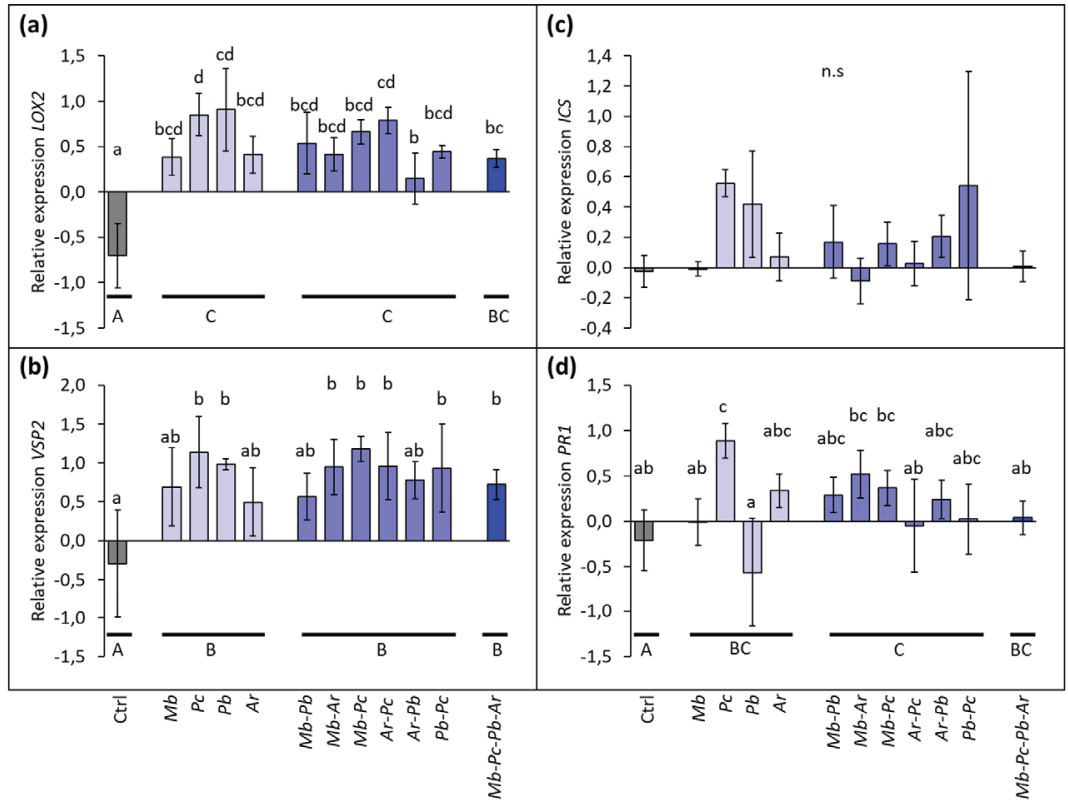

Fig. S2. Relative gene expression of Brassica nigra leaves at $48 \mathrm{~h}$ after infesting them with an increasing species richness (1, 2 or 4 species) of the leaf chewers Mamestra brassicae $(M b)$, Phaedon cochleariae $(P C)$, Pieris brassicae $(P b)$, Athalia rosae $(A r)$ alone (Chew.1), in pairs of 2 species (Chew.2), the four species (Chew.4) or untreated plants (Ctrl). We measured the expression of JA biosynthesis and JA responsive genes LOX2, and VSP2 (a) and (b), and the SA-biosynthesis and SA-responsive genes ICS and PR1 (c) and (d). Bars represent mean $\pm \mathrm{SE}$ of log transformed data. Gene expression is relative to the expression level of two reference genes GAPDH and SAR1A. Bars not sharing letters are significantly different from each other (LM, post hoc LSD). Upper case letters above the bars represent differences between treatments. "n.s" not significant. Lower case letters bellow bar-groups represent differences between species richness levels. 
Gene expression after $96 \mathrm{~h}$ of leaf chewer attack

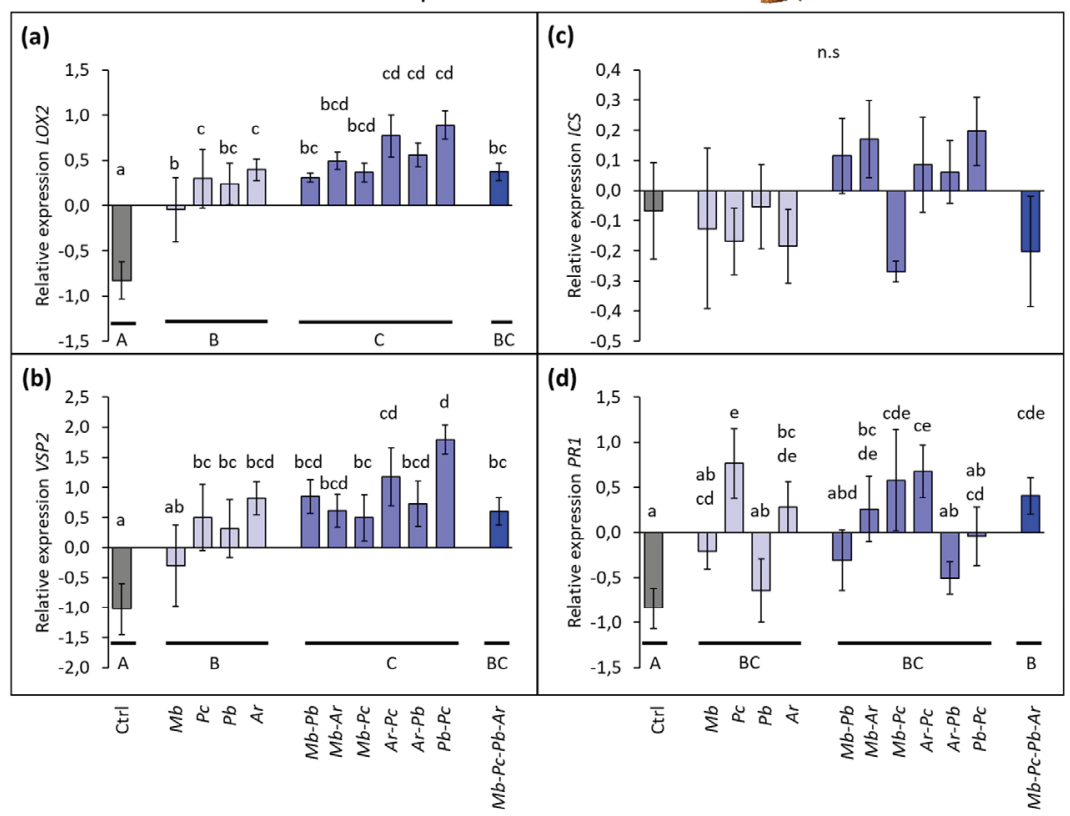

Fig. S3. Relative gene expression of Brassica nigra leaves at $96 \mathrm{~h}$ after infesting them with an increasing species richness (1, 2 or 4 species) of the leaf chewers Mamestra brassicae $(M b)$, Phaedon cochleariae $(P C)$, Pieris brassicae $(P b)$, Athalia rosae (Ar) alone (Chew.1), in pairs of 2 species (Chew.2), the four species (Chew.4) or untreated plants (Ctrl). We measured the expression of JA-biosynthesis and JA-responsive genes $L O X 2$, and VSP2 (a) and (b), and the SA-biosynthesis and SA-responsive genes ICS and PR1 (c) and (d). Bars represent mean \pm SE of log transformed data. Gene expression is relative to the expression level of two reference genes GAPDH and SAR1A. Bars not sharing letters are significantly different from each other (LM, post hoc LSD). "n.s" not significant. Upper case letters above the bars represent differences between treatments. Lower case letters bellow bar-groups represent differences between species richness levels. 
Gene expression after $48 \mathrm{~h}$ of phloem feeder attack
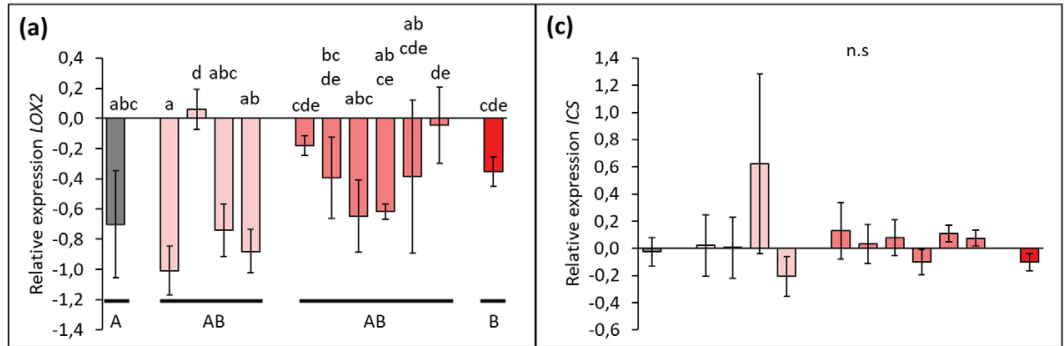

\section{(b)}

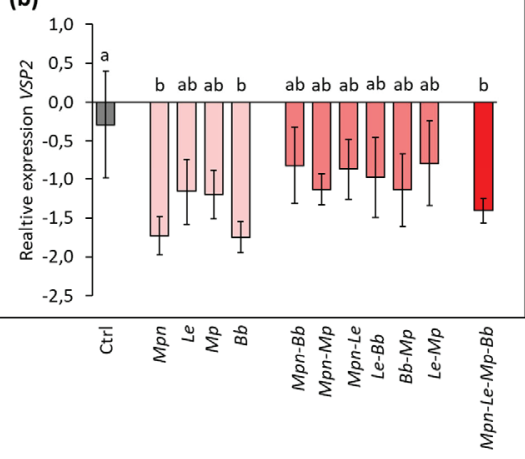

(d)
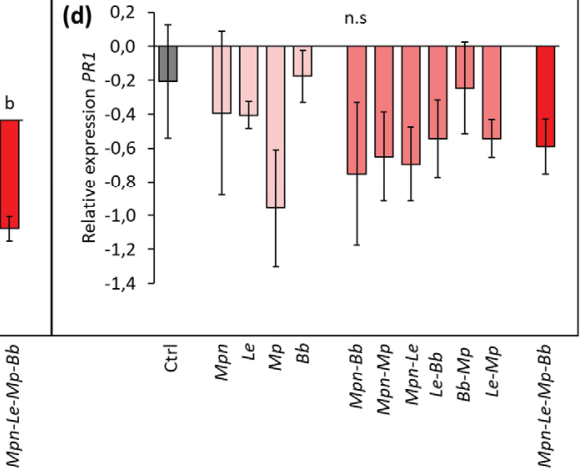

Fig. S4. Relative gene expression of Brassica nigra leaves at $48 \mathrm{~h}$ after infesting them with an increasing species richness (1, 2 or 4 species) of the phloem-feeding aphids Myzus persicae sub. nicotianae (Mpn), Lipaphis erysimi (Le), Myzus persicae (Mp), Brevicoryne brassicae (Bb), alone (Phlo.1), in pairs of 2 species (Phlo.2), the four species (Phlo.4) or untreated plants (Ctrl). We measured the expression of JA-biosynthesis and JA-responsive genes $L O X 2$, and VSP2 (a) and (b), and the SA-biosynthesis and SA-responsive genes ICS and PR1 (c) and (d). Bars represent mean \pm SE of log transformed data. Gene expression is relative to the expression level of two reference genes GAPDH and SAR1A. Bars not sharing letters are significantly different from each other (LM, post hoc LSD). "n.s" not significant. Upper case letters above the bars represent differences between treatments. Lower case letters bellow bar-groups represent differences between species richness levels. 
Gene expression after $96 \mathrm{~h}$ of phloem feeder attack

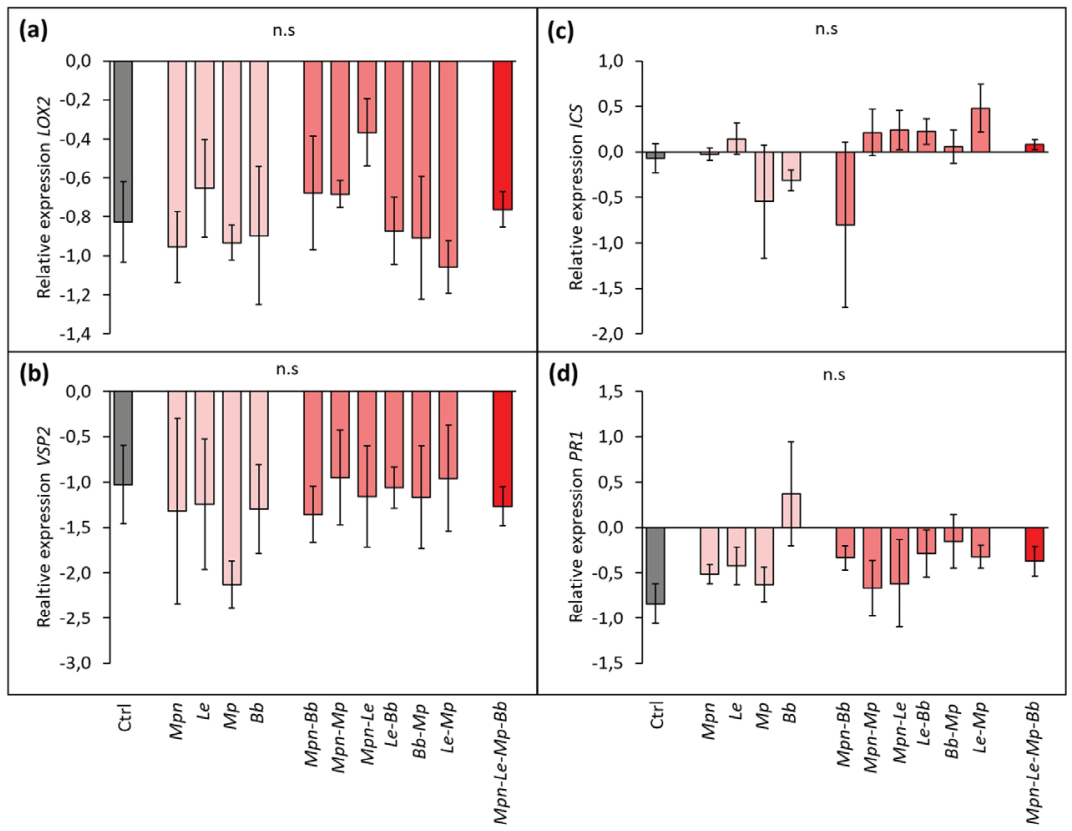

Fig. S5. Relative gene expression of Brassica nigra leaves at $96 \mathrm{~h}$ after infesting them with an increasing species richness (1, 2 or 4 species) of the phloem feeding aphids Myzus persicae sub. nicotianae (Mpn), Lipaphis erysimi (Le), Myzus persicae (Mp), Brevicoryne brassicae (Bb), alone (Phlo.1), in pairs of 2 species (Phlo.2), the four species (Phlo.4) or untreated plants (Ctrl). We measured the expression of JA-biosynthesis and JA-responsive genes $L O X 2$, and VSP2 (a) and (b), and the SA-biosynthesis and SA-responsive genes ICS and PR1 (c) and (d). Bars represent mean \pm SE of log transformed data. Gene expression is relative to the expression level of two reference genes GAPDH and SAR1A. Bars not sharing letters are significantly different from each other (LM, post hoc LSD). "n.s" not significant. Upper case letters above the bars represent differences between treatments. Lower case letters bellow bar-groups represent differences between species richness levels. 
Table S1. Overview of insect combinations for the performance mix species richness experiment for approach 1. showing the number of plant replicates per species richness. We prepared a total of eight plants for each individual insect combination. For Mix.2 we did all the possible insect combinations. For Mix.4 we selected 12 out of the 36 possible combinations (underlined and bold). We did two consecutive sub experiments, preparing half of the replicates for each sub experiment. $B b=$ Brevicoryne brassicae, $M p=$ Myzus persicae, $M p n=$ Myzus persicae sub. nicotianae, Le $=$ Lipaphis erysismi, Ar $=$ Athalia rosae, $P b=$ Pieris brassicae, $P c=$ Phaedon cochleariae, Mb=Mamestra brassicae.

\begin{tabular}{|c|c|c|c|c|}
\hline $\begin{array}{c}\text { Con.0 } \\
1 \text { treatment } \\
10 \text { plants }\end{array}$ & & & $\begin{array}{r}N \\
12 \operatorname{tre} \\
96 \\
\end{array}$ & $\begin{array}{l}4 \\
\text { ments } \\
\text { ants }\end{array}$ \\
\hline \multirow[t]{3}{*}{ Ctrl } & $\begin{array}{c}B b-M b \\
B b-A r \\
B b-P b \\
B b-P c\end{array}$ & $\begin{array}{c}M p n-M b \\
M p n-A r \\
M p n-P b \\
M p n-P c\end{array}$ & $\begin{array}{c}\underline{B b-M p-M b-A r} \\
B b-M p-M b-P b \\
B b-M p-M b-P c \\
B b-M p-A r-P b \\
B b-M p-A r-P c \\
\underline{B b-M p-P b-P c}\end{array}$ & $\begin{array}{c}M p-M p n-M b-A r \\
\text { Mp-Mpn-Mb-Pb } \\
M p-M p n-M b-P c \\
M p-M p n-A r-P b \\
\text { Mp-Mpn-Ar-Pc } \\
M p-M p n-P b-P c\end{array}$ \\
\hline & $\begin{array}{l}M p-M b \\
M p-A r \\
M p-P b \\
M p-P C\end{array}$ & $\begin{array}{l}\text { Le-Mb } \\
\text { Le-Ar } \\
\text { Le-Pb } \\
\text { Le-PC }\end{array}$ & $\begin{array}{l}B b-M p n-M b-A r \\
B b-M p n-M b-P b \\
\underline{B b-M p n-M b-P c} \\
\underline{B \boldsymbol{B b}-M p n-A r-P \boldsymbol{b}} \\
B b-M p n-A r-P C \\
B b-M p n-P b-P c\end{array}$ & $\begin{array}{c}M p-L e-M b-A r \\
M p-L e-M b-P b \\
M p-L e-M b-P c \\
\frac{M p-L e-A r-P b}{M p-L e-A r-P c} \\
M p-L e-P b-P c\end{array}$ \\
\hline & & & $\begin{array}{c}B b-L e-M b-A r \\
\text { Bb-Le-Mb-Pb} \\
B b-L e-M b-P c \\
B b-L e-A r-P b \\
\underline{B b-L e-A r-P \boldsymbol{c}} \\
B b-L e-P b-P c\end{array}$ & $\begin{array}{c}\text { Mpn-Le-Mb-Ar } \\
M p n-L e-M b-P b \\
M p n-L e-M b-P c \\
M p n-L e-A r-P b \\
M p n-L e-A r-P c \\
\text { Mpn-Le-Pb-Pc }\end{array}$ \\
\hline
\end{tabular}


Table S2. Overview of plant replicates per treatment and per species richness within each feeding guild for the approach 2: direct comparison of Plutella xylostella performance on phloem feeder, chewer and mixed herbivore induced Brassica nigra plants. Leaf chewers: $M b=$ Mamestra brassicae, Ar $=$ Athalia rosae, $P b=$ Pieris brassicae, $P c=$ Phaedon cochleariae . Phloem feeders: $B b=$ Brevicoryne brassicae, Mp= Myzus persicae, Mpn= Myzus persicae sub. nicotianae, Le $=$ Lipaphis erysismi.

\begin{tabular}{|c|c|c|c|c|}
\hline Group & Species richness & Treatment & Rep. & Replicates group \\
\hline Control & 0 & Con.0 & $\underline{24}$ & 24 \\
\hline Chewer & 4 & Chew. 4 & 24 & 24 \\
\hline Phloem feeder & 4 & Phlo. 4 & 24 & $\underline{24}$ \\
\hline \multirow{12}{*}{ Mix } & \multirow{12}{*}{4} & $B b-M p-M b-A r$ & 2 & \multirow{12}{*}{24} \\
\hline & & $B b-M p-P b-P c$ & 2 & \\
\hline & & $B b-M p n-M b-P c$ & 2 & \\
\hline & & $B b-M p n-A r-P b$ & 2 & \\
\hline & & $B b-L e-M b-P b$ & 2 & \\
\hline & & $B b-L e-A r-P c$ & 2 & \\
\hline & & $M p-M p n-M b-P b$ & 2 & \\
\hline & & $M p-M p n-A r-P c$ & 2 & \\
\hline & & $M p-L e-M b-P c$ & 2 & \\
\hline & & $M p-L e-A r-P b$ & 2 & \\
\hline & & Mpn-Le-Mb-Ar & 2 & \\
\hline & & $M p n-L e-P b-P c$ & 2 & \\
\hline
\end{tabular}


Table S3. Primer sequences for the molecular analysis of Brassica nigra genes of interest and reference genes.

\begin{tabular}{|c|c|c|c|}
\hline Gene & Pathway & AT & Sequence (Forward, reverse) \\
\hline $\begin{array}{l}\text { LIPOXIGENASE } 2 \\
\text { LOX2 }\end{array}$ & $\begin{array}{c}\mathrm{JA} \\
\text { biosynthesis }\end{array}$ & 62 & $\begin{array}{l}\text { TGCTCGTGCACGCCAGAGTC } \\
\text { AGCCAGCCCCCTGCTGATGA }\end{array}$ \\
\hline $\begin{array}{c}\text { VEGETATIVE STORAGE PROTEIN } 2 \\
\text { VSP2 }\end{array}$ & $\begin{array}{l}\text { JA } \\
\text { responsive }\end{array}$ & 58 & $\begin{array}{c}\text { TCTACGCCAAAGGACTTG } \\
\text { CTCWGTCCCGTATCCATATTGAG }\end{array}$ \\
\hline $\begin{array}{c}\text { ISOCHORISMATE SYNTHASE } \\
\text { ICS }\end{array}$ & $\begin{array}{c}\text { SA } \\
\text { biosynthesis }\end{array}$ & 58 & $\begin{array}{l}\text { GCTTGCACAGTTACAGAG } \\
\text { CACGCTCTATCTCCATATCAC }\end{array}$ \\
\hline $\begin{array}{c}\text { PATHOGENESIS-RELATED } \\
\text { PROTEIN } 1 \\
\text { PR-1 }\end{array}$ & $\begin{array}{l}\text { SA } \\
\text { responsive }\end{array}$ & 60 & $\begin{array}{l}\text { CGCCGACGGACTAAGAGGCG } \\
\text { ACACCTCGCTTTGCCACATCCA }\end{array}$ \\
\hline WRKY7O & SA crosstalk & 62 & $\begin{array}{l}\text { ATGCTTCHTGYGACAACGAC } \\
\text { TTTGTTGCCTTGCACCCTTG }\end{array}$ \\
\hline $\begin{array}{c}\text { SECRETION ASSOCIATED RAS } \\
\text { RELATED GTPASE 1A } \\
\text { SAR1A }\end{array}$ & Reference & 60 & $\begin{array}{l}\text { ATCTCTAGCCACCGTTCCCT } \\
\text { TTCCTGACGATGCTGCACAT }\end{array}$ \\
\hline $\begin{array}{c}\text { GLYCERALDEHYDE-3-PHOSPHATE } \\
\text { DEHYDROGENASE } \\
\text { GAPDH }\end{array}$ & Reference & 62 & $\begin{array}{l}\text { GGAGCTGCCAAGGCTGTCGG } \\
\text { CCTTCAGATTCCTCCTTGATAGCC }\end{array}$ \\
\hline $\begin{array}{l}\text { ACTIN-2 } \\
\text { ACT-2 }\end{array}$ & Reference & 62 & $\begin{array}{l}\text { ACATTGTGCTCAGTGGTGGA } \\
\text { TCTGCTGGAATGTGCTGAGG }\end{array}$ \\
\hline $\begin{array}{l}\text { BETA-TUBULINE } \\
\quad B-T U B\end{array}$ & Reference & 62 & $\begin{array}{l}\text { GTCAAGTCCAGCGTCTGTGA } \\
\text { TCACACGCCTGAACATCTCC }\end{array}$ \\
\hline $\begin{array}{l}\text { ELONGATION FACTOR-1 } \\
\text { EF-1 }\end{array}$ & Reference & 60 & $\begin{array}{l}\text { CGTCCCCATCTCTGGATTCG } \\
\text { ACAACCATACCGGGCTTGAG }\end{array}$ \\
\hline $\begin{array}{l}\text { PEROXIDASE } 4 \\
\text { PER4 }\end{array}$ & Reference & 62 & $\begin{array}{l}\text { TATCCTCTGCAGCCTCCTCA } \\
\text { ACACACAGACTGAAGCGTCC }\end{array}$ \\
\hline
\end{tabular}


Table S4. Variance components of the random factors of the statistical models used for testing for differences on performance of $P$. xylostella.

\begin{tabular}{|c|c|c|c|c|c|c|}
\hline \multirow{2}{*}{ Variance components } & \multicolumn{3}{|c|}{ With tray } & \multicolumn{3}{|c|}{ Without tray } \\
\hline & $s^{2}$ & Wald Z & $P$ & $s^{2}$ & Wald Z & $P$ \\
\hline Block & 0.00901 & 1.14 & 0.1269 & 0.00920 & 1.19 & 0.1167 \\
\hline Tray & 0.00808 & 3.87 & $P<0.0001$ & & & \\
\hline Plant & 0.00223 & 2.22 & 0.0131 & $\underline{0.00715}$ & 5.45 & $P<0.0001$ \\
\hline Error(aphids) & 0.08366 & 20.3 & $P<0.0001$ & 0.08194 & 20.10 & $P<0.0001$ \\
\hline Error(chewers) & 0.05074 & 22.74 & $P<0.0001$ & 0.05096 & 22.51 & $P<0.0001$ \\
\hline Error(mix) & 0.09018 & 23.27 & $P<0.0001$ & 0.09107 & 22.94 & $P<0.0001$ \\
\hline
\end{tabular}


Table S5. Significance differences of Plutella xylostella weight on plants previously attacked by a mix of leaf chewers and phloem feeders for approach 1. Insect combinations not sharing letters have a significant different effect on Plutella xylostella growth (MLM, Tukey post-hoc). Combination of inducers that affected P. xylostella growth (compared to control, untreated plants) are marked in bold and with an asterisk. Chewers: Mamestra brassicae $(M b)$, Phaedon cochleariae $(P C)$, Pieris brassicae $(P b)$, Athalia rosae (Ar). Phloem feeding aphids Myzus persicae sub. nicotianae (Mpn), Lipaphis erysimi (Le), Myzus persicae (Mp), Brevicoryne brassicae $(B b)$.

\begin{tabular}{|c|c|}
\hline Treatment & Significance \\
\hline Control & bcde \\
\hline$B b-A r$ & $a b$ \\
\hline$B b-M b$ & abcde \\
\hline$B b-P b$ & cdef \\
\hline$B b-P c$ & abcd \\
\hline Le-Ar & $a b c$ \\
\hline$L e-M b$ & cdef \\
\hline$L e-P b$ & $a b c d$ \\
\hline$L e-P C$ & $a b c$ \\
\hline$M p-A r$ & bcde \\
\hline$M p-M b$ & bcdefg \\
\hline Mpn-Ar* & fg \\
\hline$M p n-M b$ & bcde \\
\hline$M p n-P b$ & bcdefg \\
\hline$M p n-P c$ & cdef \\
\hline$M p-P b$ & cdef \\
\hline$M p-P c$ & bcdefg \\
\hline$B b-L e-A r-P c$ & cdef \\
\hline$B b-L e-P b-M b$ & $a b$ \\
\hline$B b-M p-M b-P c$ & ef \\
\hline$B b-M p n-A r-M b$ & def \\
\hline$B b-M p n-P b-P c$ & bcdef \\
\hline$B b-M p-P b-A r^{*}$ & $\mathbf{a}$ \\
\hline$M p-L e-A r-M b$ & cdef \\
\hline$M p-L e-P b-P c$ & abcde \\
\hline$M p-M p n-A r-P C$ & $a b c$ \\
\hline$M p-M p n-P b-M b$ & bcdef \\
\hline$M p n-L e-M b-P c^{*}$ & f \\
\hline$M p n-L e-P b-A r$ & bcdef \\
\hline
\end{tabular}




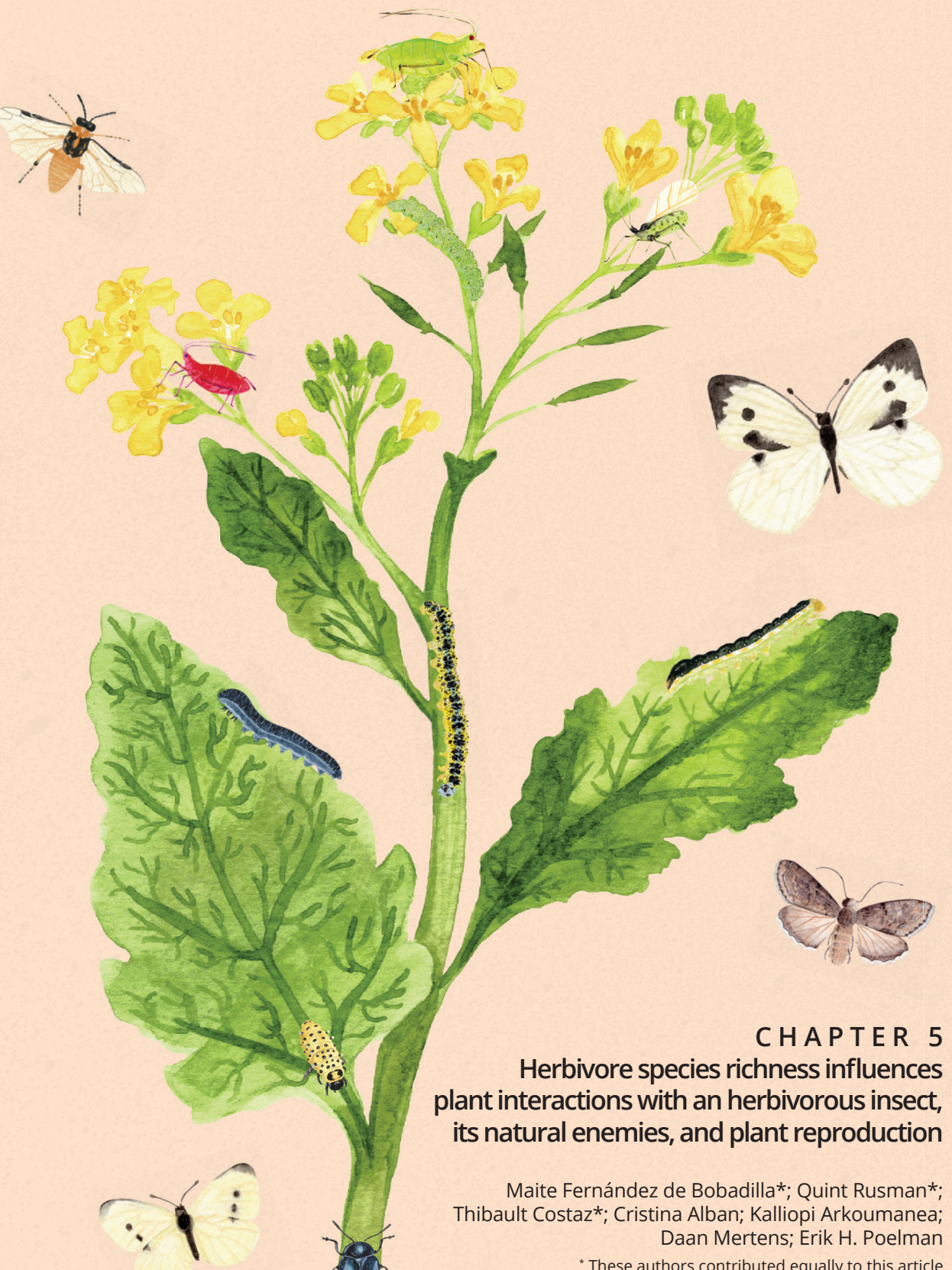

Maite Fernández de Bobadilla*; Quint Rusman*; Thibault Costaz*; Cristina Alban; Kalliopi Arkoumanea; Daan Mertens; Erik H. Poelman * These authors contributed equally to this article 



\section{ABSTRACT}

A main challenge in ecology is to assess how loss of species and consequently species interactions affect ecosystem stability. This knowledge is especially relevant within the context of insect decline that planet Earth faces. Here, we studied how insect herbivore species richness and trait composition of these species affect plant interactions with other insect community members (i.e. a herbivore, its parasitoids and pollinators) and whether these interactions affect plant fitness. We show that herbivore richness, its composition of functional traits and specific herbivore species affected the abundance of Plutella xylostella, the parasitism rate of $P$. xylostella and pollinator visitation to Brassica nigra plants. The composition of the introduced herbivore community affected plant fitness directly via reducing plant biomass that predicted resource investment into reproductive organs and these effects were stronger than fitness consequences of herbivore-induced plant-mediated interactions with other community members. Fitness effects of indirect interactions often included a network of multiple organisms and could be mediated by plant traits. All of these interactions involved effects of herbivores on pollinator visitation that closely correlated with the total number of seeds produced by a plant. For a species-rich community of phloem feeders as well as for the leaf chewer rich community seed set was reduced via a chain of interactions that reduced pollinator visitation. The effects of herbivore richness and functional traits in herbivore communities on plant interactions with subsequent herbivores, parasitoids and pollinators show how complex interaction networks on individual plants may determine plant fitness. 


\section{INTRODUCTION}

Planet Earth is facing rapid changes in ecosystem dynamics and species interactions. Important drivers of these changes are human overpopulation, climate change, habitat loss and species extinction (Tylianakis et al., 2006, 2008). The loss of integral parts of ecosystems, such as the worldwide insect decline, has caused rising concerns (Althaus et al., 2021; Hallmann et al., 2017; Halsch et al., 2020; Harvey et al. 2020; Wagner et al., 2021; Warren et al., 2020). An inherent consequence of species loss is a reduction in the number of ecological interactions which may result in reduced ecosystem stability and secondary extinctions (Soliveres et al., 2016; Tylianakis et al., 2008). Experimental and modelling studies have identified that loss of individual species in insect communities not only has cascading effects on other community members (Sanders et al., 2013, 2018), but also affects plant reproductive success (Lankau \& Strauss, 2008; Soper Gorden $\&$ Adler, 2018). The cascading effects of interaction loss on plant fitness signals evolutionary consequences of species loss for plant trait selection (Zeng \& Wiens, 2020). To understand and predict changes in selection regimes on plant traits by declines of organisms of higher trophic levels, a major challenge is identifying which insect groups and interactions more strongly affect plant fitness. This includes exploring direct and indirect interactions among antagonistic and mutualistic community members that may further modulate fitness outcomes of plant interactions with their insect community (Ando et al., 2017; Poelman \& Kessler, 2016; Rusman et al. 2019a).

In terrestrial ecosystems, plants are at the basis of many food chains being the food source for a species-rich community of insect herbivores (Schoonhoven, van Loon \& Dicke, 2005) with $85 \%$ of plants also providing pollen and nectar to a diversity of flower-visiting insects (Ollerton et al., 2011). Consequently, plants are central in a network of species that interact directly or indirectly via the plant (Ohgushi, 2005). The high diversity of organisms interacting with a plant creates a playground of interacting selective agents on the plant (e.g. herbivores, parasitoids or pollinators, abiotic stress and competition for resources with other plants). Due to the complexity of the plant-associated 
network, prediction of selection on plant traits is not straightforward (Strauss, 2014). Indeed, diffuse evolution of plant traits is often observed, where selection on traits is not only explained by one-to-one species interactions, but rather is the effect of the network of interactions (Strauss, 2014; Poelman \& Kessler 2016). Important selective agents on plant traits are herbivores, natural enemies of the herbivores and pollinators. Herbivores can directly affect plant fitness by consuming photosynthetic plant tissues, flowers and/or seeds and indirectly by altering the attraction of other herbivores, natural enemies of the herbivores and by altering pollinator visitation (Grass et al., 2018; Rusman et al. 2020). Natural enemies of the herbivores, such as parasitoids and predators, can have positive direct effects on plant fitness by reducing herbivore pressure (Bustos-Segura et al., 2020) and indirect effects by altering herbivore, omnivore and pollinator behaviour. Interactions with pollinators strongly affect plant fitness directly, because pollinators mediate plant reproduction. The increase in seed number may in turn attract seed-feeding herbivores (Pérez-Barrales et al., 2013; Steenhuisen \& Johnson, 2012). Thus, direct and indirect interactions between herbivores and other community members affect the insect community associated with a plant, including other herbivores (Poelman et al., 2008), parasitoids (Blubaugh et al., 2018; Bukovinszky et al., 2012), pollinators (Chrétien et al., 2021; Rusman et al., 2018), and can affect plant fitness (Cozzolino et al., 2015; Soper Gorden \& Adler, 2018). However, it is poorly understood how plants maximise fitness when defending against attack by diverse assemblages of herbivores while maintaining the attraction of parasitoids/predators and pollinators.

Here, we study how herbivore species richness and its trait composition affect plant interactions with its insect community and whether these interactions affect plant fitness. We conducted a manipulative field study where we subjected Brassica nigra plants to attack by an insect herbivore community with increasing species richness from one and/or two feeding guilds (i.e. phloem feeders, leaf chewers or a mix of both). Recent studies identified that B. nigra plants respond differentially to attack by different herbivore feeding 
guilds as well as the number of species attacking the plant (Fernández de Bobadilla et al., 2021), and that indirect interactions due to herbivore-induced plant responses affect plant reproductive success (Pashalidou et al., 2015; Rusman et al., 2018, 2020). We specifically studied how the composition of the introduced herbivore community affected a) plant colonisation by the herbivore Plutella xylostella, one of the most prevalent herbivores attacking B. nigra plants (Mertens et al., 2021); b) top-down control of this herbivore population by parasitoids; c) visitation of flowers by pollinators; and d) how these interactions collectively affect plant fitness in terms of seed production. We examined the relations between the different community members and effects of varying levels of herbivore richness and feeding guild by applying structural equation modelling (SEM). This allowed us to partition the direct and indirect effects of different community members on plant fitness, and how early-season herbivore attack alters these relationships. We consider as direct effects those where initial herbivory induces plant phenotypic changes that result in effects on plant fitness, whereas indirect effects are herbivoreinduced effects on other recorded community members that have fitness consequences. By assessing the direct and indirect effects of herbivore community composition on important members of a plant-associated insect community this work sheds light on which interactions more strongly affect plant fitness, and potentially impose selection on plant traits.

\section{MATERIALS AND METHODS}

\section{Plants and insects}

Seeds of black mustard (B. nigra, Brassicales: Brassicaceae) were obtained from a natural population in the vicinity of Wageningen, The Netherlands $\left(51^{\circ}\right.$ $57^{\prime} 32^{\prime \prime}$ N, $5^{\circ} 40^{\prime} 23^{\prime}$ E). Seeds were sown on peat soil (Lentse Potgrond) and germinated in a greenhouse at $22 \pm 2{ }^{\circ} \mathrm{C}, 60-70 \% \mathrm{RH}$ and 16:8 h L:D. One week after germination, the plants were transplanted into peat soil cubes and planted in the field when three weeks old. One week before planting in the field, the plants were acclimatized to field conditions in an open greenhouse (i.e. without climate control). 
Eight insect species that commonly attack B. nigra were used to manipulate the composition of the starting herbivore community on individual plants (Table 1). We used four chewing herbivore species: the turnip sawfly, Athalia rosae (Ar) (Hymenoptera: Tenthredinidae); the mustard leaf beetle, Phaedon cocbleariae (Pc) (Coleoptera: Chrysomelidae); the cabbage moth, Mamestra brassicae $(M b)$ (Lepidoptera: Noctuidae); and the large cabbage white, Pieris brassicae $(P b)$ (Lepidoptera: Pieridae). We used four phloem-feeding herbivore species: the cabbage aphid, Brevicoryne brassicae (Bb); the mustard aphid, Lipaphis erysimi (Le); the green peach aphid, Myzus persicae (Mp); and the tobacco aphid, Myzus persicae sub. nicotianae (Mpn) (all Hemiptera: Aphididae) (Table 1). Mamestra brassicae, P. brassicae, B. brassicae and P. xylostella were reared on Brussels sprouts plants (Brassica oleracea L. var. gemmifera cv. Cyrus). Myzus persicae, M. persicae sub. nicotianae, L. erysimi, A. rosae and P. cocbleariae were reared on radish (Raphanus sativus). All insects were obtained from the stock rearing of the Laboratory of Entomology, Wageningen University kept under greenhouse conditions $(22 \pm 2$ ${ }^{\circ} \mathrm{C}, 60-70 \%$ RH and 16:8 h L:D).

Table 1. Insects used for the experiment as inducers, in brackets abbreviations used throughout the document.

\begin{tabular}{|c|c|c|l|}
\hline Species (Abbreviation) & Picture & Order & Feeding guild \\
\hline Athalia rosae $(\mathrm{Ar})$ & & Hymenoptera & Leaf chewer \\
Mamestra brassicae $(\mathrm{Mb})$ & Lepidoptera & Leaf chewer \\
Phaedon cochleariae $(\mathrm{PC})$ & Coleoptera & Leaf chewer \\
Pieris brassicae (Pb) & & Lepidoptera & Leaf chewer \\
Brevicoryne brassicae (Bb) & & Hemiptera & Phloem feeder \\
Lipaphis erysimi (Le) & & Hemiptera & Phloem feeder \\
Myzus persicae (Mp) & & Hemiptera & Phloem feeder \\
\hline
\end{tabular}




\section{Plant treatments}

The effect of species richness and trait composition and trait richness of the herbivore community on plant interactions with herbivores, parasitoids and pollinators and on plant seed production were studied in the Droevendaal experimental fields of Wageningen University ( $51^{\circ} 59^{\prime} 26.5^{\prime \prime} \mathrm{N}, 5^{\circ} 39^{\prime} 50.5^{\prime}$ ' E) during the summer of 2019. We planted 800 plants divided over 5 blocks: each block was a field of $21 \times 17 \mathrm{~m}$ with 160 plants planted on the same day at $1.5 \mathrm{~m}$ distance from each other. Blocks were planted separately every two weeks between the $6^{\text {th }}$ of May and $1^{\text {st }}$ of July 2019 (Fig. 1).

Two days after planting in the field, we infested plants with different herbivore communities by varying species richness of herbivores (one, two or four herbivore species) and their trait composition in terms of the feeding guilds they belong to (leaf-chewing, phloem-feeding, or a mix of both). This resulted in 13 treatments: control plants without herbivore treatment (Con- 0$)$, four different treatments with one chewing herbivore species (Chew-1), one treatment with the four chewing herbivore species combined (Chew-4), four different treatments with one phloem-feeding herbivore species (Phlo-1), one treatment with the four phloem-feeding herbivore species combined (Phlo-4), mixture of one chewing and one phloem-feeding herbivore species (Mix-2), mixture of two chewing and two phloem-feeding herbivore species (Mix-4). We excluded species richness of three species, because of the imbalance that these treatments would have in numbers of leaf-chewer and phloem-feeder species. In each block, we included 12 replicates per herbivore combination for the chewer and phloem feeder treatments (Chew-1, Chew-4, Phlo-1 Phlo-4). For the Mix-2 and Mix-4 treatments, we included one replicate for each herbivore combination: Mix-2 resulted in 16 insect combinations, while for Mix-4 a selection of 12 of the 36 species combinations were included. Combinations were selected to have the largest differences between species combinations, while each herbivore is present the same number of times (6 times) (Fernández de Bobadilla et al., 2021). For a complete list of all species combinations see Table 2 . All treatments were randomised per block. 


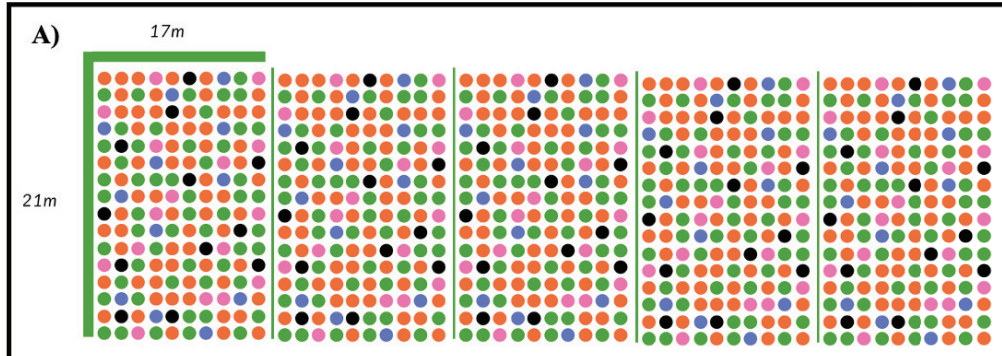

B)

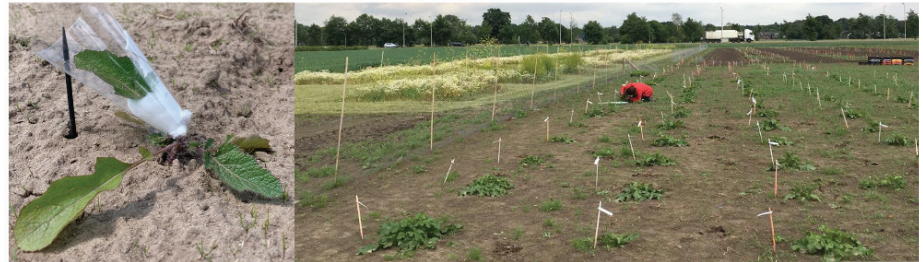

C)
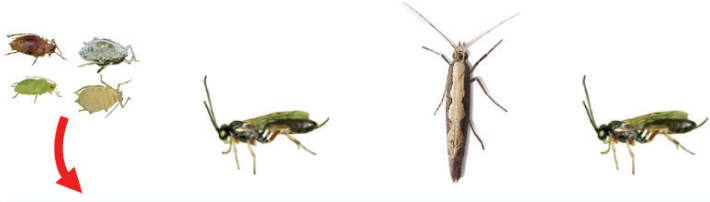

Day 2

Day 21

Day 14

Day 21

Day 28

Induction

Parasitism

Abundance

Parasitism

Abundance

P. xylostella

P. xylostella

P. xylostella

P. xylostella

Time point 2

Time point 1

Time point 2

Time point 2

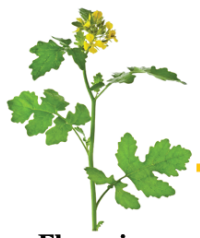

Flowering

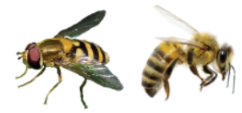

Day 7

Pollinator

observation

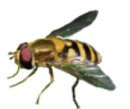

Day 14

Pollinator

observation

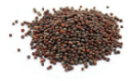

Seed set

Figure 1. Experimental setup. A) Field setup: 5 blocks, each colour represents one herbivore treatment. B) Experiment time line of the measurements 
Table 2. Overview of insect combinations for the mixed species richness treatments. For Mix-2 we did all the possible insect combinations. For Mix-4 we selected 12 out of the 36 possible combinations (underlined and bold). $B b=$ Brevicoryne brassicae, Mp=Myzus persicae, Mpn $=$ Myzus persicae sub. nicotianae, Le $=$ Lipaphis erysismi, Ar= Athalia rosae, $P b=$ Pieris brassicae, $P c=$ Phaedon cochleariae, Mb=Mamestra brassicae.

\begin{tabular}{|c|c|c|c|}
\hline \multicolumn{2}{|c|}{$\begin{array}{c}\text { Mix-2 } \\
16 \text { treatments }\end{array}$} & \multicolumn{2}{|c|}{$\begin{array}{c}\text { Mix-4 } \\
12 \text { treatments } \\
\end{array}$} \\
\hline $\begin{array}{l}B b-M b \\
B b-A r \\
B b-P b \\
B b-P c\end{array}$ & $\begin{array}{c}M p n-M b \\
M p n-A r \\
M p n-P b \\
M p n-P c\end{array}$ & $\begin{array}{c}\text { Bb-Mp-Mb-Ar } \\
B b-M p-M b-P b \\
B b-M p-M b-P c \\
B b-M p-A r-P b \\
B b-M p-A r-P c \\
\underline{B b-M p-P b-P c}\end{array}$ & $\begin{array}{c}M p-M p n-M b-A r \\
M p-M p n-M b-P b \\
M p-M p n-M b-P c \\
M p-M p n-A r-P b \\
M p-M p n-A r-P c \\
M p-M p n-P b-P C\end{array}$ \\
\hline $\begin{array}{l}M p-M b \\
M p-A r \\
M p-P b \\
M p-P c\end{array}$ & $\begin{array}{l}L e-M b \\
L e-A r \\
L e-P b \\
L e-P C\end{array}$ & $\begin{array}{l}B b-M p n-M b-A r \\
B b-M p n-M b-P b \\
\text { Bb-Mpn-Mb-Pc} \\
\text { Bb-Mpn-Ar-Pb} \\
B b-M p n-A r-P c \\
B b-M p n-P b-P c\end{array}$ & $\begin{array}{l}M p-L e-M b-A r \\
M p-L e-M b-P b \\
\frac{M p-L e-M b-P c}{M p-L e-A r-P b} \\
\frac{M p-L e-A r-P c}{M p-L e-P b-P c}\end{array}$ \\
\hline & & $\begin{array}{c}B b-L e-M b-A r \\
\text { Bb-Le-Mb-Pb} \\
B b-L e-M b-P c \\
B b-L e-A r-P b \\
\text { Bb-Le-Ar-Pc} \\
B b-L e-P b-P c\end{array}$ & $\begin{array}{c}\text { Mpn-Le-Mb-Ar } \\
M p n-L e-M b-P b \\
M p n-L e-M b-P c \\
M p n-L e-A r-P b \\
M p n-L e-A r-P c \\
\text { Mpn-Le-Pb-Pc }\end{array}$ \\
\hline
\end{tabular}

In all herbivore communities the starting density consisted of four individuals for leaf chewer treatments and eight individuals for phloem feeder treatments, divided over one or four species. For example, Chew-1 included four individuals of the same leaf chewer species, while Chew-4 included one individual of each leaf chewer species. For the mixed treatments, we used two leaf-chewer individuals plus four phloem-feeder individuals, divided over two or four species. For chewing herbivores, first-instar larvae were placed on the youngest fully expanded leaf of the plant, while for phloem-feeding herbivores we used wingless adults. Insects could freely move and feed from their preferred feeding sites on the plant. The number of species and densities of inducers were based on field observations of insect communities of B. nigra plants in the same area (Mertens et al., 2021). 


\section{Natural colonisation of $P$. xylostella}

To investigate the effects of trait composition and species richness of the herbivore community on subsequent interactions with the herbivore community, we monitored natural colonisation of the herbivore P. xylostella. Plutella xylostella is one of the dominant herbivores colonising brassicaceous plants in the vegetative state. We recorded the number of larvae of P. xylostella that naturally colonised individual plants of each of the 13 herbivore community treatments including plants that were not experimentally infested with herbivores. Plants were checked for caterpillar presence at two time points, two and four weeks after planting them in the field. We did so by carefully checking each leaf for the presence of caterpillars of P. xylostella (L2-L4). To further support the field observations on caterpillar presence, we tested moth oviposition preference for plants infested with the different herbivore communities in a greenhouse setup (Fig. S1, Tables S1 and S3).

\section{Parasitism of P. xylostella}

To investigate the effects of trait composition and species richness of the herbivore community on the top-down control of P. xylostella by parasitoids, we monitored natural parasitism of caterpillars of $P$. xylostella on plants infested with each of the 13 herbivore community treatments including plants that were not infested with herbivores. We placed 15 L2-L3 P. xylostella larvae on a young leaf of each plant, nine and eighteen days after the initial induction. The leaf petiole was covered with some cotton and surrounded with a transparent plastic sheet shaped as a funnel to prevent the caterpillars from escaping (Fig. 1). The caterpillars were placed five days before assessing the abundance of P. xylostella (see previous section) to ensure that, in the rare case of a caterpillar escaping, these would have pupated and would not be counted as natural colonisation of P. xylostella. The caterpillars were placed at 10 a.m. and, two days later, at 5 p.m., caterpillars were recollected, transferred to Petri dishes and stored in a refrigerator $\left(5^{\circ} \mathrm{C}\right)$ or in a freezer $\left(-20^{\circ} \mathrm{C}\right)$ for longer storage until dissection. The caterpillars were dissected and checked for the presence of parasitoid eggs using a stereomicroscope (Olympus SZ51 KL-300 LED). The parasitoid eggs

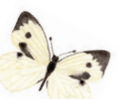


or larvae were not identified to the species level. However, field studies show that the most common parasitoids of P. xylostella caterpillars in The Netherlands are Diadegma spp. (Bukovinszky et al., 2004).

\section{Pollinator visitation}

To investigate effects of trait composition and species richness of the herbivore community on pollinator visitation, we recorded pollinator visitation on plants infested with each of the 13 herbivore community treatments including plants that were not infested with herbivores. Recordings were done at two time points: a) between 7 and 9 days and b) between 14 and 16 days after plants had started flowering. Pollinator visitation to a plant was monitored for $10 \mathrm{~min}$. When a pollinator arrived to a plant and had contact with a flower, the identity of the pollinator and the number of flowers visited were recorded (Rusman et al., 2018, 2020). The identity of other pollinators that visited the plant during this observation was recorded as well. If the same pollinator individual returned to the plant under observation after having visited a different plant, we scored that visit as a new visit (Rusman et al., 2018, 2020). Recordings were done during the day (between 9 a.m. and 1 p.m., or 2 p.m. and 5 p.m.) only when weather conditions were favourable for pollinator activity $\left(15-30{ }^{\circ} \mathrm{C}\right.$ and wind speed $\leq 6 \mathrm{~m} / \mathrm{s}$ (Rusman et al., 2018, 2020), using a handheld computer (Psion Workabout Protm 3) programmed with The Observer XT software (version 10, Noldus Information Technology, Wageningen, The Netherlands).

\section{Plant phenotype and seed production}

To investigate the effects of herbivore-induced changes on plant phenotype and assess whether these changes affect plant interactions with the insect community, we measured multiple plant parameters: the number of leaves, plant height, plant diameter and percentage of leaf damage. Measurements were taken after assessing the abundance of P. xylostella (see previous section), using a tape measure. The percentage of leaf damage on a full plant scale was visually estimated on a percentage scale (0-100\%). 
Plants were monitored every 2-3 days for the start and end of the flowering period. Plants were considered flowering when the first flower opened and considered finished with flowering when all buds and flowers were gone, with only developing and ripe siliques remaining on flower stalks (Rusman et al., 2020). We calculated the duration of flowering by subtracting the number of days needed to start flowering from the number of days to the termination of flowering (Rusman et al., 2020).

Life-time seed production was assessed by seed number and weight. First harvesting date for each plant was selected before the first siliques would lose their seeds (Rusman et al., 2018; 2020). At first harvest, we collected all ripe siliques and left immature siliques and flowers on the plant. Then, plants were checked weekly and all siliques were harvested when ripe. Siliques were stored in paper bags in a dry storage room until seeds were manually extracted from the siliques. We calculated the total number of seeds per plant by weighing 100 seeds, and the total weight of seeds harvested per plant (Rusman et al., 2018, 2020). We estimated the total number of seeds by dividing total seed weight by the weight of 100 seeds and multiplied the result by 100 . The weight of one seed was estimated by dividing the weight of 100 seeds by 100 .

\section{Statistical analysis}

The effects of species richness and trait composition of the herbivore community were first analysed separately for plant interactions with P. xylostella, parasitoids, pollinators, and plant traits (flowering traits, seed production) using generalized linear mixed modelling, GL(M)M. For count data such as number of insects (e.g. number of herbivores, parasitoid eggs, or pollinators), flowers visited, days (from infestation, since flowering), and seed numbers, we used GL(M)M with a Poisson distribution and a log link function, or negative binomial distribution with a log link function to correct for overdispersion when necessary. For continuous data such as seed weight, we used linear (mixed) models with a Gaussian distribution and

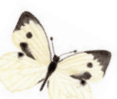


identity link function or a Gamma distribution with a $\log$ link function if the data did not follow a normal distribution. Two models were fitted to the data: the first model included herbivore treatment and time point as fixed factors. The second model included herbivore species richness (treatments assigned to 0,1, 2 or 4 species), herbivore feeding guild (treatments assigned to control, chewing, phloem-feeding, or combination), and time point as fixed factors. Non-significant interactions $(\mathrm{P}>0.05)$ were excluded from the model. Random factors were selected using a backward approach: all random factors such as block, day (only for pollinators), time (morning or afternoon; only for pollinators) were added to the models and removed if they explained $<3 \%$ of the variation or were statistically non-significant ( $P$ $>$ 0.05). Model assumptions were assessed graphically using the Pearson residuals against the fitted value by the models as well as against all the included covariates (i.e. fixed factors). Pairwise comparisons were evaluated using Tukey's post hoc test for significant variables $(\mathrm{P}<0.05)$. For comparisons within treatment and combined herbivore species richness * feeding guild we used non-adjusted $\mathrm{p}$ values to cope with the high number of treatments. For comparisons within herbivore species richness and feeding guild we used adjusted p values. For number of parasitized P. xylostella recaptured, an offset with the natural logarithm of the total number of recaptured $P$. xylostella larvae was added to the model to account for differences in the total number of larvae recaptured per plant. Plants for which no P. xylostella larvae were recovered were not included in the analysis as they do not provide information on the prevalence of parasitism of $P$. xylostella larvae. Unfortunately, larvae from the fifth block were lost due to unexpected storage failure by malfunction of the freezer and could not be included in the analysis. For plant seed production, plants that produced $\leq 2,500$ or $>40,000$ seeds were considered artifacts. Removing these outliers affected all treatments equally (no overall effect of herbivory; $p=0.431$ for plants with 0 to 2,500 seeds). In addition, plants from block 5 were excluded due to extremely high mortality (almost 80\%). We used the lme4 (Bates et al., 2015), 
multcomp (Hothorn et al., 2008), lsmeans (Lenth, 2016), lmtest (Zeileis and Hothorn, 2002), emmeans (Russell, 2018), ggplot2 (Wickham, 2016) packages for these analyses.

We performed structural equation modelling (SEM) to examine the effects of the herbivore treatment on relations between plant traits and plant interactions with P. xylostella and their natural enemies, pollinators, and plant fitness. We built three models: one including each herbivore species treatment, one including herbivore species richness, and one including herbivore feeding guild. We did this to identify effects of individual herbivore species, as well as broader patterns based on herbivore richness or herbivore feeding guild. All included variables are displayed in figure 14. The variables height and radius were used as independent variables instead of dependent variables because of model identification problems. For parasitism rate and number of pollinators and flowers visited we only used data from time point 1 , because time point 2 contained too few observations. We only used total pollinator visits and total flowers visited because including individual pollinator species/groups reduced the number of observations. All variables were checked for normality and homogeneity of variance, and if violated, variables were log-transformed. Since transformation did not solve assumption violations in all cases, we used the "Yuan-Bentler" test statistic. Good model fit was verified by inspecting the robust Test statistic $(P>0.05)$, CFI $(>0.9)$, RMSEA $(<0.08)$, RMSEA Low $(<0.01)$, RMSEA High $(<0.1)$, SRMR $(<0.08)$. We used the lavaan package for these analyses (Rosseel 2012). All analyses were performed in $\mathrm{R}$ version 4.0.2 ( $\mathrm{R}$ Core Team, 2020).

\section{RESULTS}

\section{Natural colonisation of Plutella xylostella is affected by herbivore richness}

The abundance of P. xylostella differed among plants induced by herbivore communities that differed in richness and species composition $\left(\mathrm{df}=1, \chi^{2}=4.67, \mathrm{P}\right.$ 


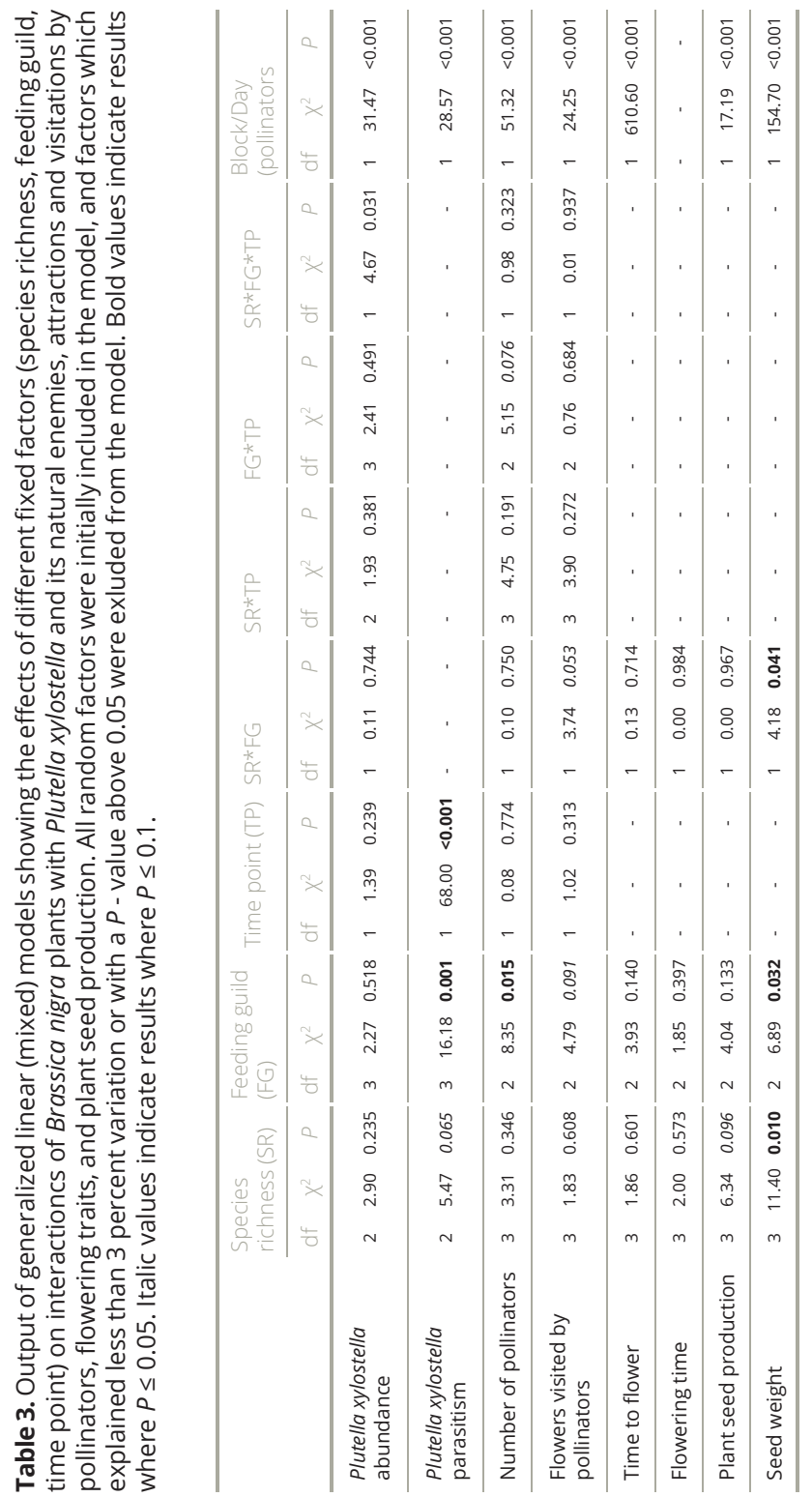


$=0.031$, Table 3). These effects were restricted to P. xylostella abundance two weeks after induction of $B$. nigra plants and not observed within one week after induction (Fig. 2). We found fewer P. xylostella caterpillars on plants that did not receive initial herbivory and on plants attacked by four leaf chewers compared with the other induction treatments. Irrespective of species richness, phloem-feeder induction made the plants more attractive to $P$. xylostella compared to untreated plants. In contrast, in the case of leaf chewers the richness of attackers was important, as plants attacked by one chewer species had a larger number of P. xylostella than plants attacked by four species of leaf chewers (Fig. 2). When plants were attacked by the highest species richness of herbivores (i.e. four species) the abundance of $P$. xylostella differed depending on the guild of the attacker. Plants attacked by four species of phloem feeders had the largest number of $P$. xylostella, followed by plants attacked by chewers and phloem feeders at the same time (i.e. Mix-4) and plants attacked by four species of chewers had the smallest number of $P$. xylostella. Likewise, in a greenhouse study, the oviposition preference of P. xylostella changed depending on the guild of the herbivores that had previously attacked the plant. Plutella xylostella laid more eggs on plants attacked by four species of phloem feeders compared with plants attacked by a mix of two phloem feeders and two chewers (i.e. Mix-4) $(\mathrm{P}<0.001$; Wilcoxon signed rank test, Fig. S2). In contrast to the overall influence of trait composition and species richness of the attacker community, the abundance of P. xylostella in the field was not affected by specific herbivores (Table 4). However, in a greenhouse study, chewer identity affected the oviposition preference of P. xylostella. The moths laid fewer eggs on plants attacked by $M$. brassicae and more eggs on plants attacked by $P$. cocbleariae compared to plants attacked by the four chewer species $(\mathrm{P}<0.05$, Wilcoxon signed rank test, Fig. S3). The oviposition preference of P. xylostella was not affected by the identity of phloem feeders nor by the richness of species in a mix with chewers and phloem feeders (Fig S4, S5). 

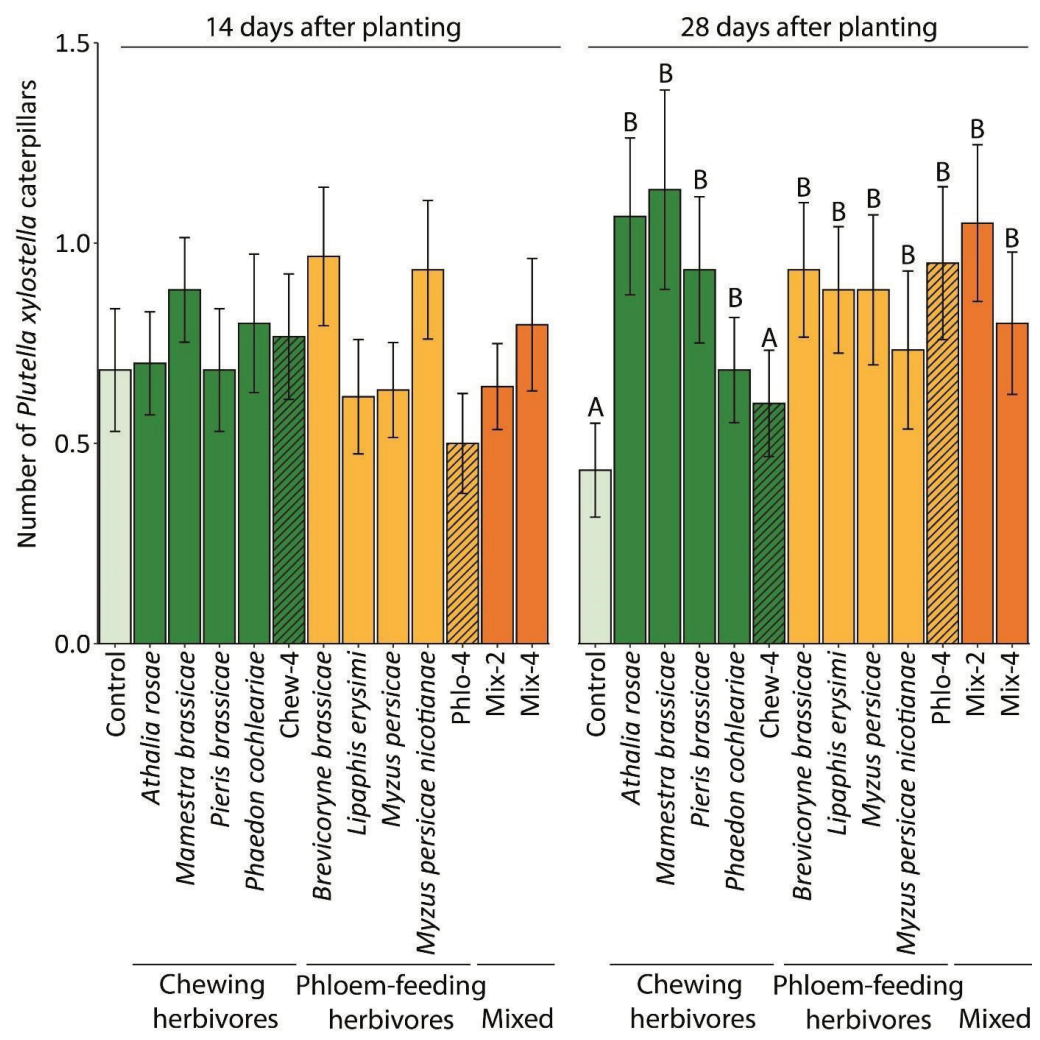

Figure 2. Abundance of Plutella xylostella larvae 14 and 28 days after planting on herbivore-treated- or on untreated plants. Bars represent the mean \pm the standard error of the mean. Capital letters above bars indicate significant differences $(P \leq 0.05)$ between herbivore treatments within a time point based on non-adjusted Tukey's post hoc tests. 


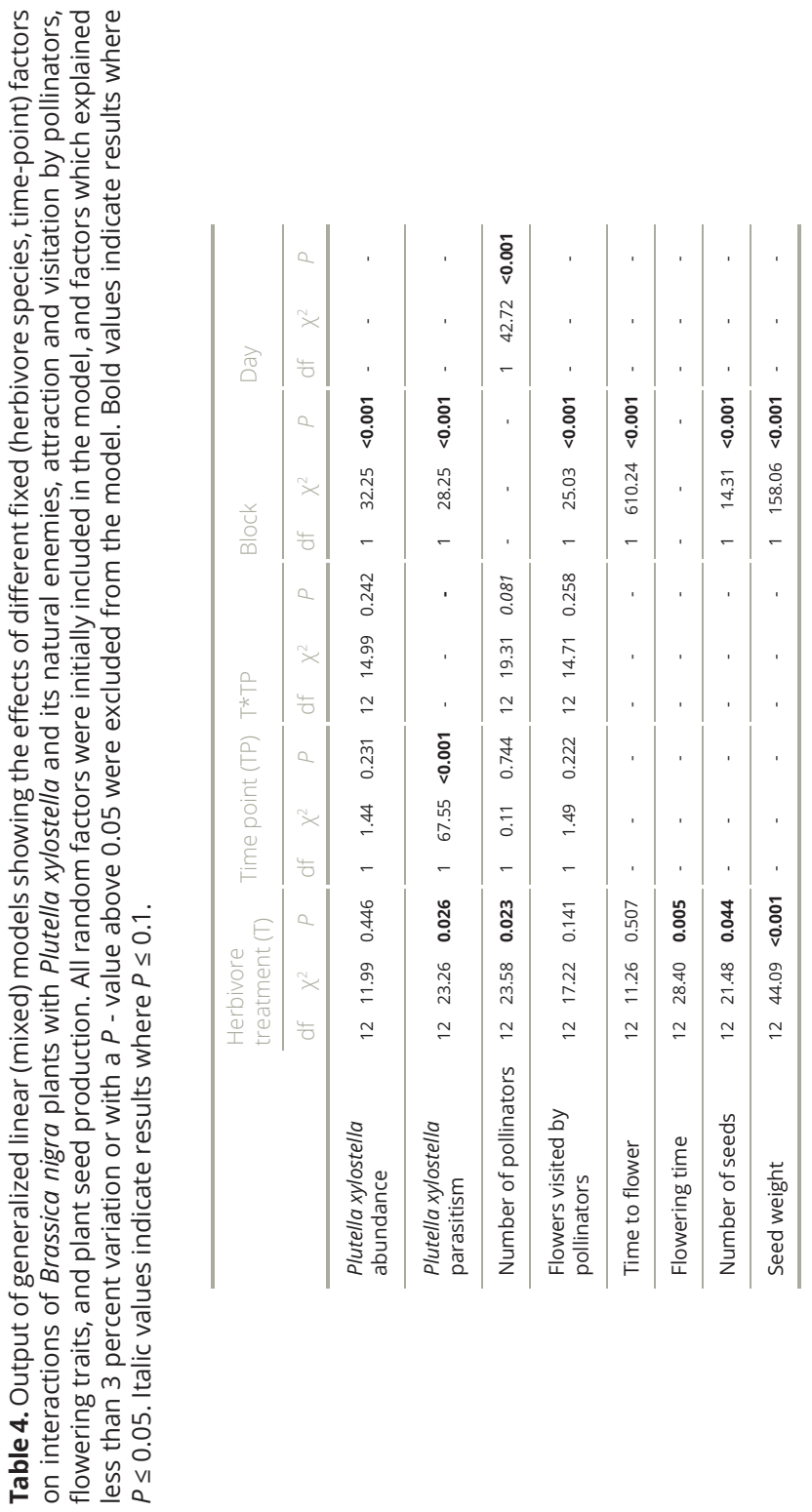




\section{Parasitism of Plutella xylostella in the field is affected by the guild of initial attackers}

The parasitism of P. xylostella was significantly different across feeding guild and time point (Feeding guild: $\mathrm{df}=3, \chi^{2}=16.18, \mathrm{P}<0.001$; time point: $\mathrm{df}=1 \chi^{2}=67.99, \mathrm{P}<0.001$; Fig. 3 , Table 3). The number of parasitised larvae was significantly higher on plants induced by a mixture of chewers and phloem feeders (i.e. Mix-4) than on plants attacked by one feeding guild alone (Fig. 3). Between leaf chewer and phloem feeder communities there was no difference in the number of parasitised P. xylostella larvae (Fig. 3). The species richness of herbivores attacking the plant marginally affected the parasitism of P. xylostella ( $\mathrm{df}=2, \chi^{2}=5.47, \mathrm{P}=0.065$; Fig. 3 and Table 3). In addition to feeding guild and richness of the attacker community, specific herbivores affected the parasitism rate of P. xylostella ( $\mathrm{df}=12, \chi^{2}=23.26, \mathrm{P}=0.026$; Fig. 4 and Table 3). The degree of P. xylostella parasitism was higher on plants attacked by P. brassicae, $M$. persicae, M. persicae sub. nicotianae and by a mix of two phloem feeders and two chewers (i.e. Mix-4) compared with untreated plants (Fig. 4). The parasitism on Mix-4 plants was higher than on plants induced by the four species of phloem feeders, and plants induced by the mix of the four species of leaf chewers. In addition to the herbivore treatments, the time of measurement also affected the parasitism rate of $P$. xylostella larvae which was higher nine days after initial induction, compared to 18 days after initial induction $\left(\mathrm{df}=1, \chi^{2}=67.55, \mathrm{P}=\right.$ 0.001; Figs. 3 and 4, Tables 3 and 4).

\section{Pollinator visitation in the field is affected by richness of initial attackers}

We observed 4077 flowering plant visits by at least 21 insect groups/ species (Table 5). Almost 80 percent of the visits were by two dominant species: Eristalis tenax and Apis mellifera. For the remaining visits we observed more than seven syrphid fly species, more than four bumblebee species, and several solitary bee, fly, butterfly and wasp species. 


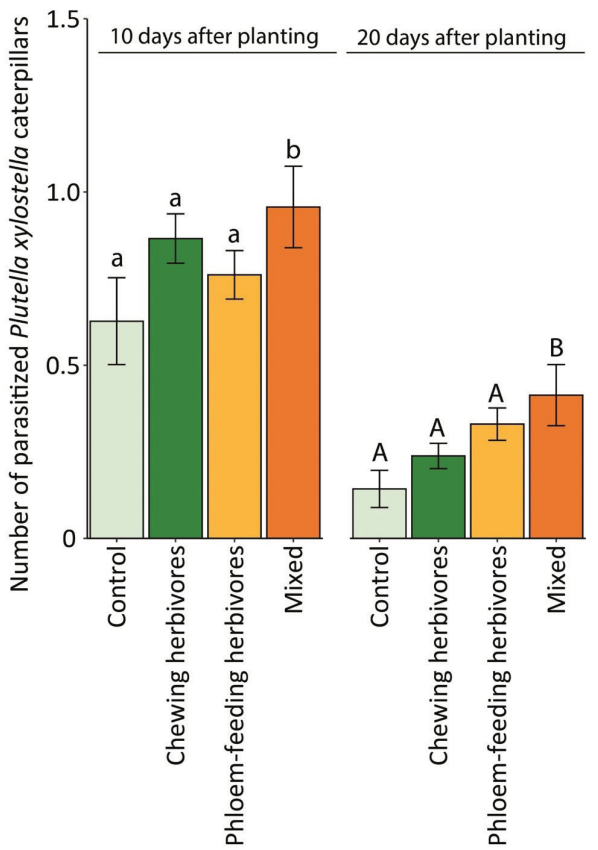

Figure 3. Number of parasitized Plutella xylostella larvae on Brassica nigra plants treated with different herbivores grouped by feeding guild and species richness. Plants were treated 2 days after planting or were left untreated at the two time points measured. Bars represent the mean \pm the standard error around the mean. Letters above bars indicate significant differences $(P \leq 0.05)$ between herbivore treatments within a time point based on adjusted Tukey's post hoc tests, small and capital letters were used for different time points. 

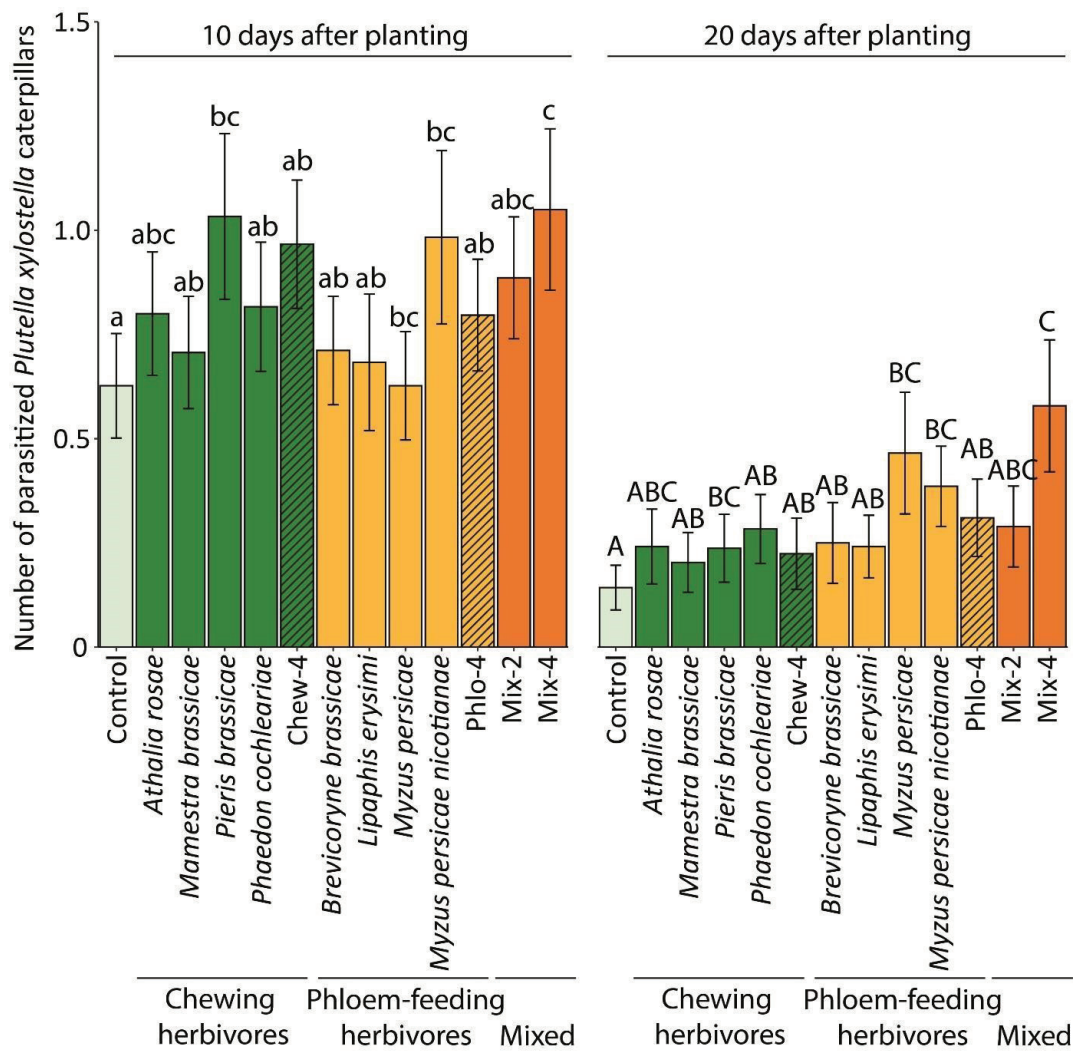

Figure 4. Number of parasitized Plutella xylostella larvae 10 or 20 days after planting on plants that received herbivore treatments or were left untreated. Bars represent the mean \pm the standard error around the mean. Letters above bars indicate significant differences $(P \leq 0.05)$ between herbivore treatments within a time point based on non-adjusted Tukey's post hoc tests, small and capital letters were used for different time points. 
Table 5. Pollinator groups/species observed visiting flowers of Brassica nigra.

\begin{tabular}{|c|c|c|c|}
\hline Pollinator groups & Species & $\begin{array}{c}\text { Total number } \\
\text { observed }\end{array}$ & $\begin{array}{c}\text { Percentage of total } \\
\text { visitors }\end{array}$ \\
\hline Honeybees & Apis mellifera & 1583 & 38.8 \\
\hline \multirow[t]{9}{*}{ Syprhid flies } & Eristalis tenax & 1594 & 39.1 \\
\hline & Eupeodes corollae & 218 & 5.3 \\
\hline & Sphaerophoria scripta & 198 & 4.9 \\
\hline & Episyrphus balteatus & 35 & 0.9 \\
\hline & Scaeva pyrastri & 29 & 0.7 \\
\hline & Helophilus pendulus & 18 & 0.4 \\
\hline & Syrphus spp. & 7 & 0.2 \\
\hline & $\begin{array}{l}\text { Elves (Meliscaeva spp., } \\
\text { Meligramma spp., Melangyna } \\
\text { spp., Fagisyrphus spp., } \\
\text { Epistrophella spp.) }\end{array}$ & 1 & 0.0 \\
\hline & other Syrphidae & 110 & 2.7 \\
\hline Solitary bees & $\begin{array}{c}\text { Andrena spp. } \\
\text { Lasioglossum spp. }\end{array}$ & 92 & 2.3 \\
\hline Flies & Calyptrata & 91 & 2.2 \\
\hline \multirow[t]{5}{*}{ Bumblebees } & Bombus lapidarius & 18 & 0.4 \\
\hline & B. pascuorum & 5 & 0.1 \\
\hline & B. terrestris & 2 & 0.0 \\
\hline & B. hypnorum & 1 & 0.0 \\
\hline & other Bombus spp. & 4 & 0.1 \\
\hline \multirow[t]{2}{*}{ Butterflies } & Pieris spp. & 10 & 0.2 \\
\hline & other Lepidoptera & 6 & 0.1 \\
\hline Wasps & Vespula spp., Polistes spp. & 16 & 0.4 \\
\hline \multirow[t]{2}{*}{ Other insects } & Unidentified & 31 & 0.8 \\
\hline & Total & 4077 & \\
\hline
\end{tabular}


Overall pollinator visitation was affected by the feeding guild of the herbivores infesting the plants (Tables 3 and 4). The effect depended marginally on time point and was more apparent two weeks after flowering than one week after flowering (Tables 3 and 4). Two weeks after flowering, plants attacked by phloem feeders received fewer pollinator visits compared to undamaged plants, to plants attacked by chewers and to plants attacked by a mix of phloem feeders and chewers (i.e. Mixed) (Fig. 5). In addition, two weeks after flowering the overall pollinator visitation was affected by specific herbivores (Fig. 6). Plants attacked by the phloem feeders $L$. erysimi or $M$. persicae sub. nicotianae were visited by fewer pollinators than plants that did not receive herbivory, than plants that were attacked by Athalia rosae, by $M$. brassicae, by Pieris brassicae, or by a mix of one chewer and one phloem feeder (Fig. 6). The overall flower visitation by pollinators was marginally affected by the interaction between herbivore richness and feeding guild (Table 3). Overall flower visitation was not affected by specific herbivores (Fig. 7). Compared to control plants, the number of flowers visited by pollinators was not different on plants attacked by one or four species of phloem feeders (i.e. Phlo-1 or Phlo-4), by four species of chewers (i.e. Chew-4) or by two or four species of a mix of chewers and phloem feeders (i.e. Mix-2, Mix-4) (Fig. 8). In contrast, the number of flowers visited by pollinators was higher on plants attacked by one leaf-chewer species than on control plants (Fig. 8).

\section{Plant phenotypic traits are affected by herbivore richness and functional traits}

Herbivore communities introduced early in the season affected flowering time, but not the onset of flowering of B. nigra plants, and effects depended on herbivore identity rather than trait composition or richness of the herbivore community (Figs. 9 and 10). M. persicae sub. nicotianae induced shorter flowering compared to any other treatment (Fig. 10). Interestingly, none of the other phloem feeders, nor the combination of the four phloem feeders, reduced flowering time compared to control plants. On the contrary, plants that were attacked by $B$. brassicae flowered longer than plants attacked by the four chewers or than plants attacked by P. brassicae (Fig. 10). 


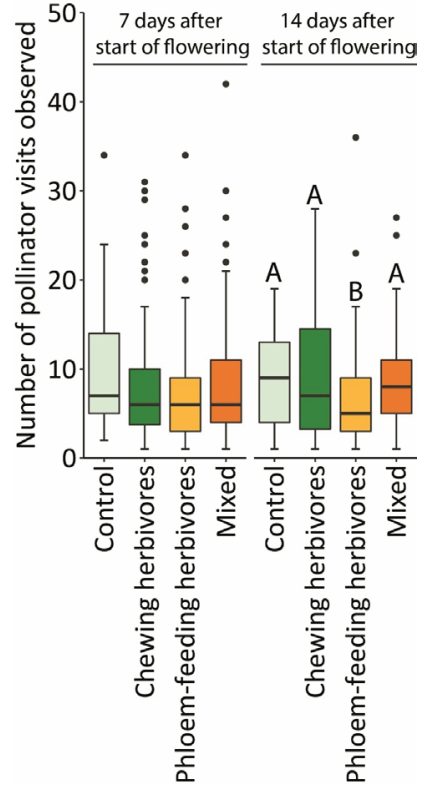

Figure 5. Number of pollinator visits observed on flowers of Brassica nigra plants treated with different herbivores grouped by feeding guild or left untreated. Boxplots show median (line), first and third quartiles, minimum and maximum. Outliers (1.5 times the interquartile range below the first or above the third quartile) are represented by circles. Observations lasted for 10 minutes and were made at two time points: between 7 and 9 days, and 14 and 16 days after plots had started flowering. Letters above bars indicate significant differences $(P \leq 0.05)$ between herbivore treatments within a time point based on adjusted Tukey's post hoc tests, small and capital letters were used for different time points. 


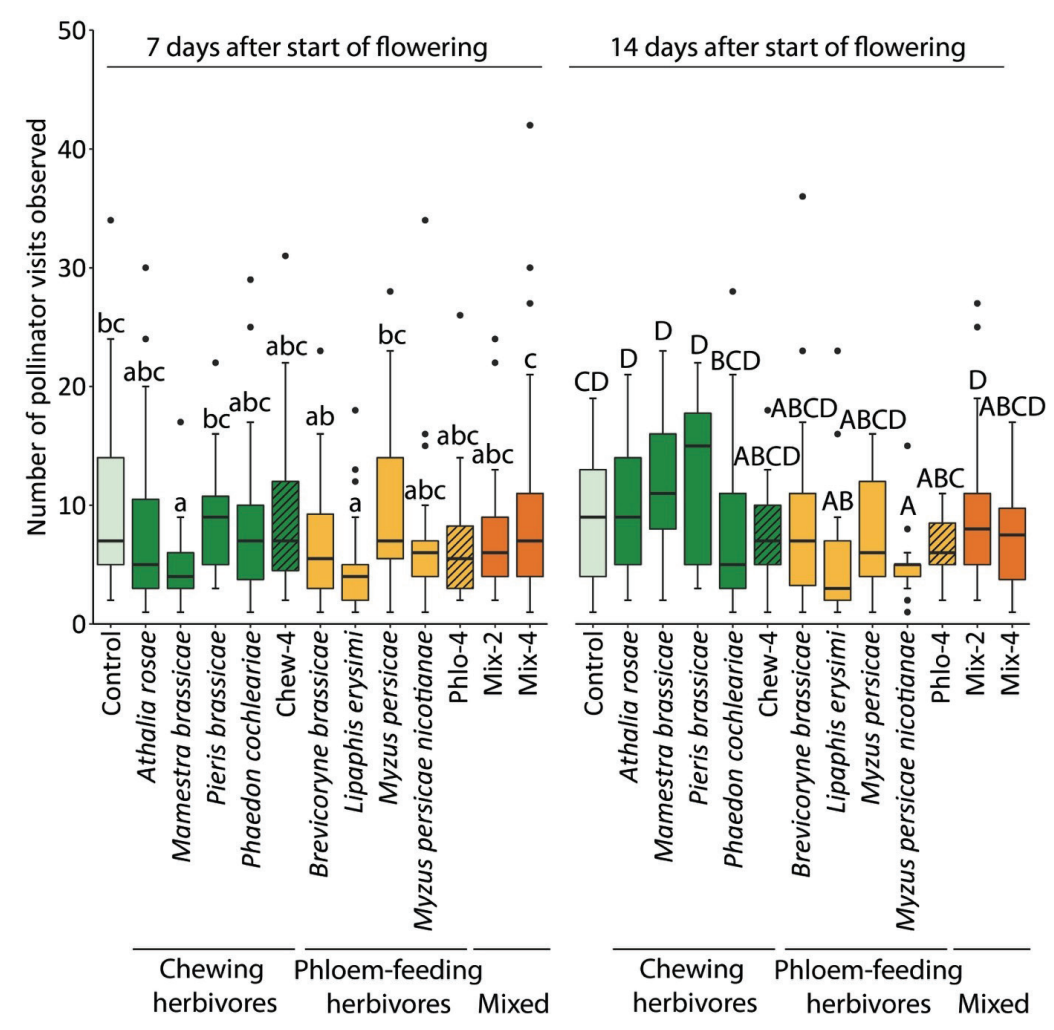

Figure 6. Number of pollinator visits observed on flowers of Brassica nigra plants treated with different herbivores or left untreated. Boxplots show median (line), first and third quartiles, minimum and maximum. Outliers (1.5 times the interquartile range below the first or above the third quartile) are represented by circles. Observations lasted for 10 minutes and were made at two time points: between 7 and 9 days, and 14 and 16 days after plots had started flowering. Letters above bars indicate significant differences $(P \leq 0.05)$ between herbivore treatments within a time point based on non-adjusted Tukey's post hoc tests, small and capital letters were used for different time points. 


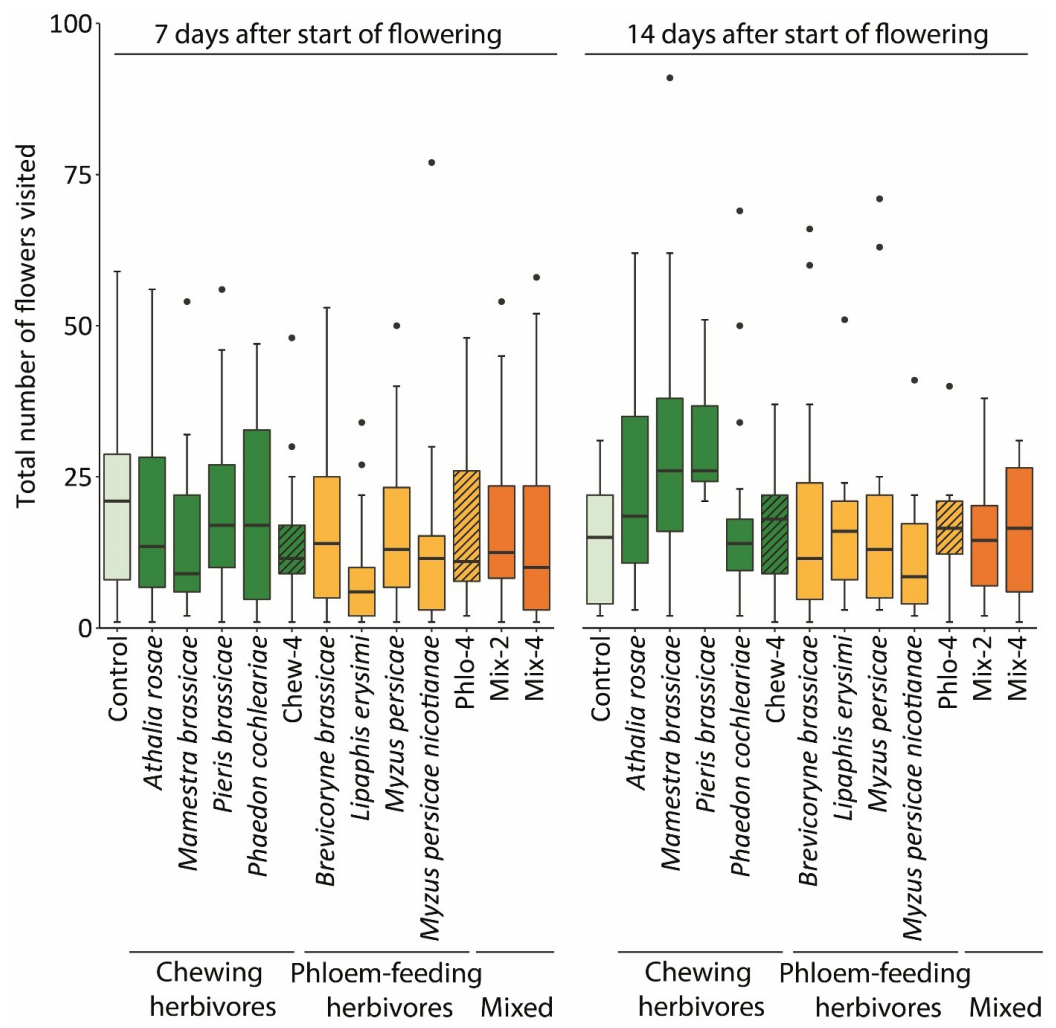

Figure 7. Number of flowers visited by all pollinators on Brassica nigra plants treated with different herbivores or left untreated. Boxplots show median (line), first and third quartiles, minimum and maximum. Outliers ( 1.5 times the interquartile range below the first or above the third quartile) are represented by circles. Observations lasted for 10 minutes and were made at two time points: between 7 and 9 days, and 14 and 16 days after plots had started flowering. Letters above bars indicate significant differences $(P \leq 0.05)$ between herbivore treatments within a time point based on non-adjusted Tukey's post hoc tests, small and capital letters were used for different time points. 


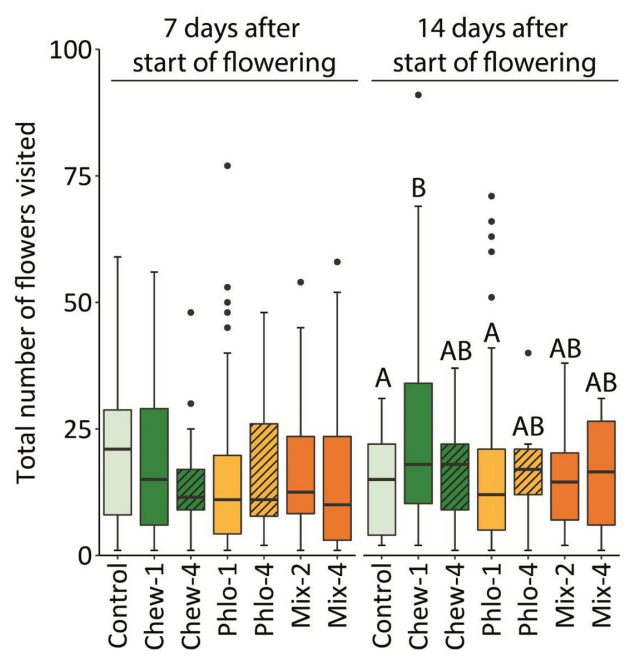

Figure 8. Number of flowers visited by all pollinators on Brassica nigra plants treated with different herbivores grouped by feeding guild and species richness or left untreated. Boxplots show median (line), first and third quartiles, minimum and maximum. Outliers (1.5 times the interquartile range below the first or above the third quartile) are represented by circles. Observations lasted for 10 minutes and were made at two time points: between 7 and 9 days, and 14 and 16 days after plots had started flowering. Letters above bars indicate significant differences $(P \leq 0.05)$ between herbivore treatments within a time point based on Tukey's post hoc tests, small and capital letters were used for different time points. 


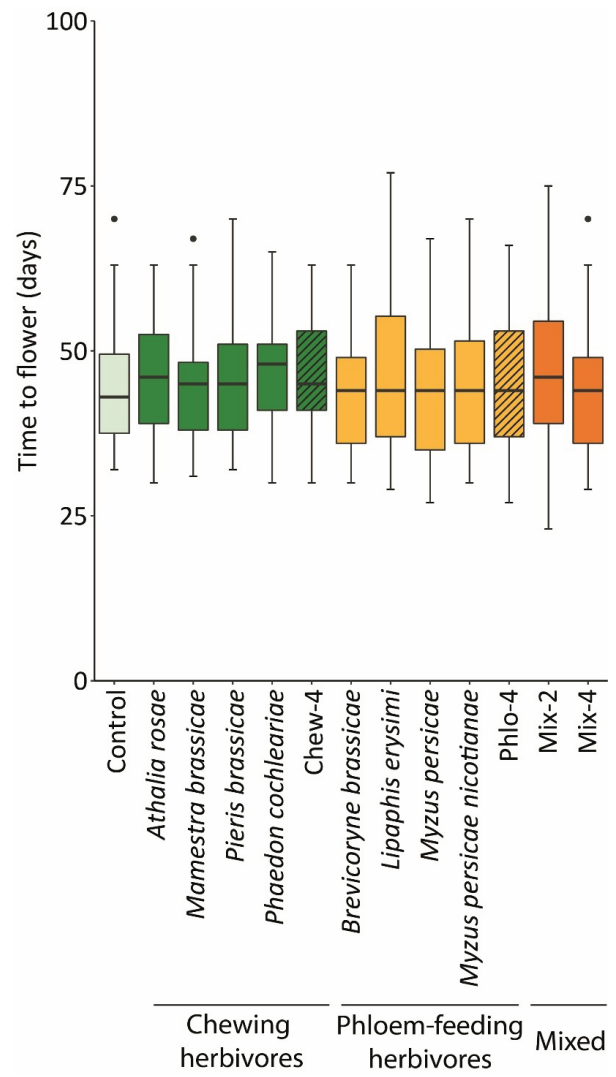

Figure 9. Time needed for Brassica nigra plants to start flowering after being treated with different herbivores or left untreated. Boxplots show median (line), first and third quartiles, minimum and maximum. Outliers ( 1.5 times the interquartile range below the first or above the third quartile) are represented by circles. 


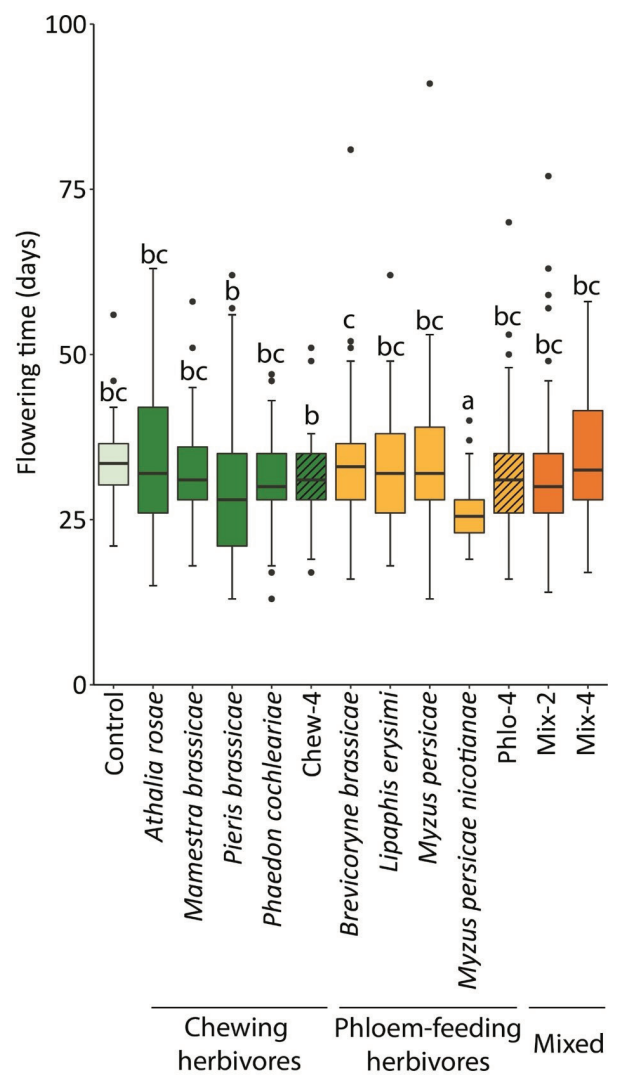

Figure 10. Flowering time of Brassica nigra plants after being treated with different herbivores or left untreated. Boxplots show median (line), first and third quartiles, minimum and maximum. Outliers (1.5 times the interquartile range below the first or above the third quartile) are represented by circles. Letters above bars indicate significant differences ( $P \leq$ 0.05 ) between herbivore treatments based on non-adjusted Tukey's post hoc tests. 


\section{Plant seed production}

The number of seeds produced by $B$. nigra plants was not directly affected by herbivore species richness ( $\mathrm{df}=3, \chi^{2}=6.34, \mathrm{P}=0.096$ ) nor by herbivore feeding guild ( $\mathrm{df}=2, \chi^{2}=4.04, \mathrm{P}=0.133$ ). However, specific herbivore species reduced the number of seeds produced ( $\mathrm{df}=12, \chi^{2}=21.48$, $\mathrm{P}=0.044$ ). Plants attacked by $A$. rosae, by Myzus persicae sub. nicotianae, and by the highest richness of chewers and phloem feeders (i.e. Chew-4 and Phlo-4 respectively) produced fewer seeds compared to plants that did not receive herbivory (Fig. 11). Seed weight was affected by herbivore species richness $\left(\mathrm{df}=3, \chi^{2}=11.40, \mathrm{P}=0.010\right)$, herbivore feeding guild $\left(\mathrm{df}=2, \chi^{2}=6.89\right.$, $\mathrm{P}=0.032)$, and by their interaction ( $\left.\mathrm{df}=1, \chi^{2}=6.34, \mathrm{P}=0.041\right)$ (Fig. 12). The richness of attackers only affected seed weight on mixed-induced plants. Plants attacked by the lowest richness of the mixture of phloem feeders and chewers (i.e. Mix-2) produced fewer seeds than plants attacked by the higher mixed species richness (i.e. Mix-4) (Fig. 12). The seed weight produced by Mix2-induced plants was lower than plants that did not receive initial herbivore treatment or than plants that were induced by one species of phloem feeder (Phlo-1) (Fig. 12). In addition to trait composition and richness of the attacker community, specific herbivores affected the seed weight produced by B. nigra plants ( $\left.\mathrm{df}=12, \chi^{2}=44.09, \mathrm{P}=0.001\right)$. Athalia rosae-treated and Mix-2-induced plants produced lighter seeds whereas B. brassicae-treated and Mix-4-induced plants produced heavier seeds (Fig. 13).

\section{Direct and indirect effects of community members on plant fitness}

Structural equation modelling (SEM) shows that the introduced herbivore communities directly and indirectly altered interactions between community members and that this change in interactions has consequences for plant fitness (Fig. 14). Plant performance parameters were strong indicators of plant reproductive success. Plants with more leaves produced more but lighter seeds (i.e. positive correlation of number of leaves with seed number but negative correlation with seed weight) (Fig. 14). Bigger plants (i.e. bigger

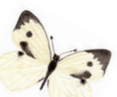




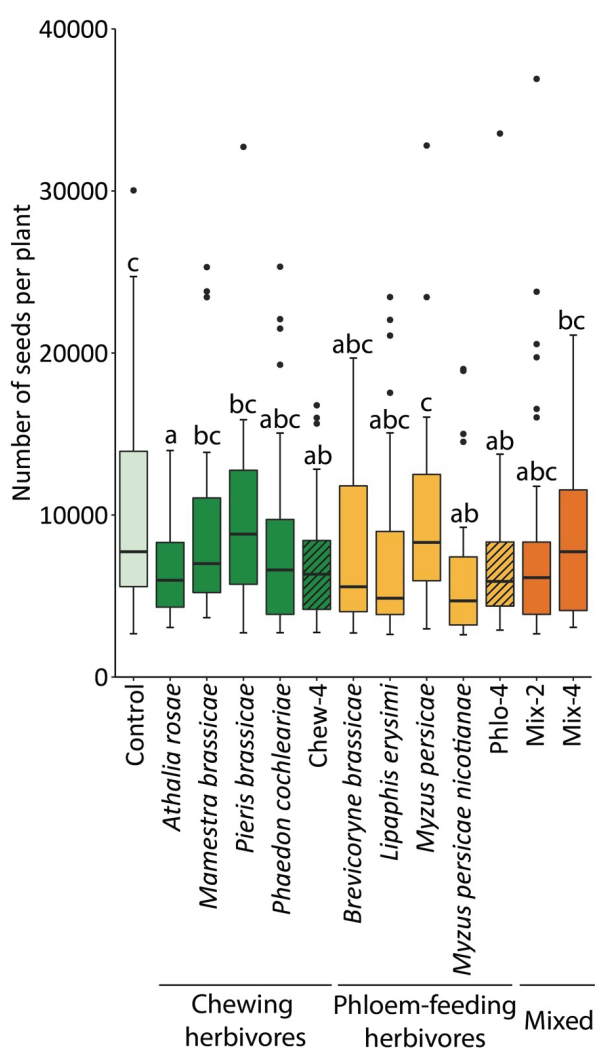

Figure 11. Number of seeds produced by Brassica nigra plants after being treated with different herbivores or left untreated. Boxplots show median (line), first and third quartiles, minimum and maximum. Outliers ( 1.5 times the interquartile range below the first or above the third quartile) are represented by circles. Letters above bars indicate significant differences $(P \leq 0.05)$ between herbivore treatments based on non-adjusted Tukey's post hoc tests. 


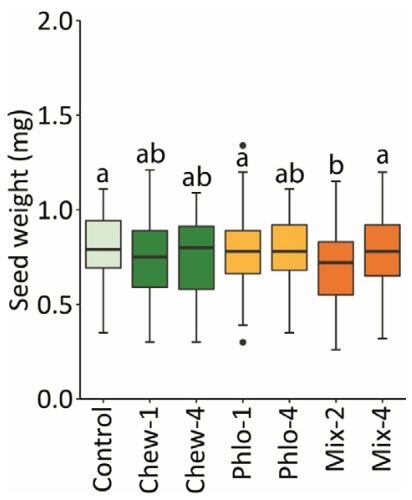

Figure 12. Weight of individual seeds produced by Brassica nigra plants after receiving different herbivore treatments grouped by species richness or feeding guild or left untreated. Boxplots show median (line), first and third quartiles, minimum and maximum. Outliers (1.5 times the interquartile range below the first or above the third quartile) are represented by circles. Letters above bars indicate significant differences $(P \leq 0.05)$ between herbivore treatments based on adjusted Tukey's post hoc tests.

plant radius) produced heavier seeds (Fig. 14). Plants with a longer duration of flowering produced more and heavier seeds (Fig. 14). The introduced herbivore communities affected plant fitness frequently by altering plant performance traits. Myzus persicae was the only herbivore with a direct positive connection with seed weight (Fig. 14). Myzus persicae-induced and B. brassicaeinduced plants produced heavier seeds explained by a reduction in the number of leaves of the plant (Fig. 14). In contrast, A. rosae and a mix of one phloem feeder and one chewer (i.e. Mix-2) had a negative effect on seed weight by reducing the duration of flowering (Fig. 14). The amount of leaf damage positively affected seed weight by increasing the duration of flowering (Fig. 14). Although the introduced herbivore communities affected abundance of P. xylostella, its parasitism rate and plant visitation by pollinators (Fig. 14), these effects were weakly connected to plant fitness parameters. The strongest connections were made through multiple interactions among organisms and were often via plant traits (Fig. 14). In itself, the abundance of P. xylostella did 


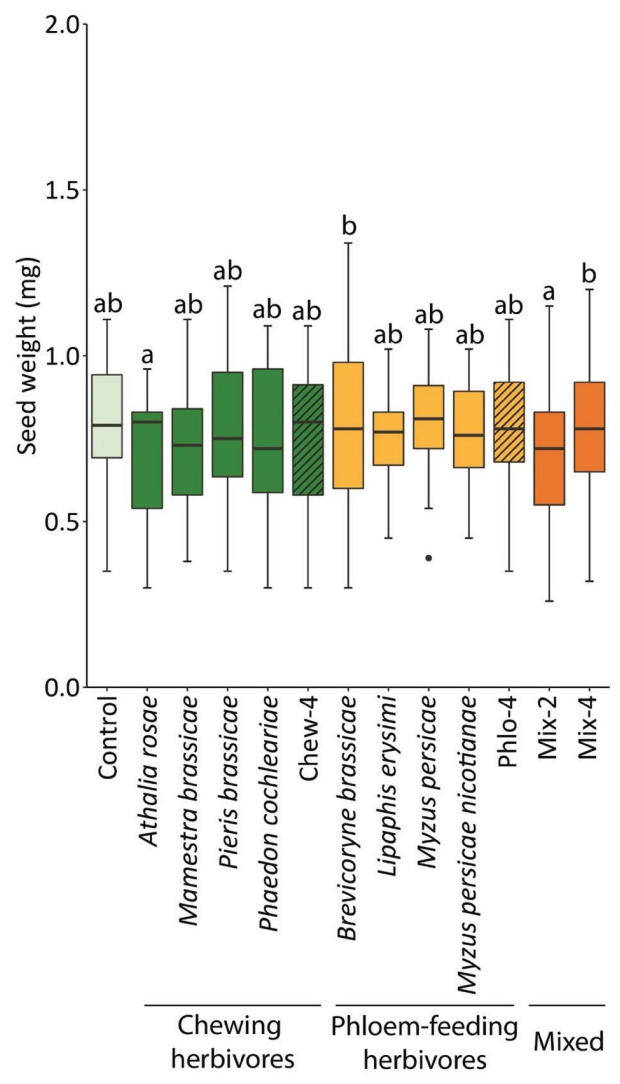

Figure 13. Weight of individual seeds produced by Brassica nigra plants after receiving the herbivore treatments or left untreated. Boxplots show median (line), first and third quartiles, minimum and maximum. Outliers (1.5 times the interquartile range below the first or above the third quartile) are represented by circles. Letters above bars indicate significant differences $(P \leq 0.05)$ between herbivore treatments based on non-adjusted Tukey's post hoc tests 
not correlate with plant fitness (number of seeds and seed weight). However, the parasitism rate of P. xylostella one week after induction negatively correlated with the number of seeds produced by the plants (Fig. 14). Pollinators were the strongest predictors for plant fitness (Fig. 14). The number of pollinators visiting the plant had a positive effect on the number of seeds produced by increasing the number of flowers visited per plant (Fig. 14). Flowering time had a negative effect on the numbers of seeds produced because it reduced the numbers of flowers visited by pollinators (Fig. 14). By interaction with pollinators, the abundance of P. xylostella and its parasitism rate affected plant fitness. Abundance of P. xylostella had an indirect positive effect on the number of seeds produced by increasing the number of flowers visited by pollinators (Fig. 14). The parasitism rate of $P$. xylostella positively correlated with the number of flower visitors, that enhanced the number of flowers visited and positively correlated with seed set. These interactions were differentially influenced by the composition of the herbivore community introduced early in the season. Introduction of a species rich phloem feeder community (Phlo-4) negatively correlated with plant damage, that corresponded with higher parasitism rates, cascading to increased number of flower visitors and visitations that enhanced seed number (Fig. 14). Thus, introduction of the phloem feeder community eventually resulted in lower plant fitness (see also Fig. 11). A similar negative effect on seed number could be deduced for the specific phloem feeder L. erysimi that reduced the number of flowers visited by pollinators (Fig. 14). Also, the species-rich leaf chewer community (Chew-4) reduced the number of flowers visited by pollinators that positively correlated with seed number, thus yielding overall reduced fitness. A mix of herbivores (Mix-2, Mix-4), three out of four leaf chewer species (A. rosae, P. cochleariae, P. brassicae) and three out of four phloem feeder species (Myzus persicae, Myzus persicae nicotianae, B. brassicae) positively affected abundance of P. xylostella that in turn positively correlated with the number of flowers visited by pollinators, which resulted in increased seed set. 


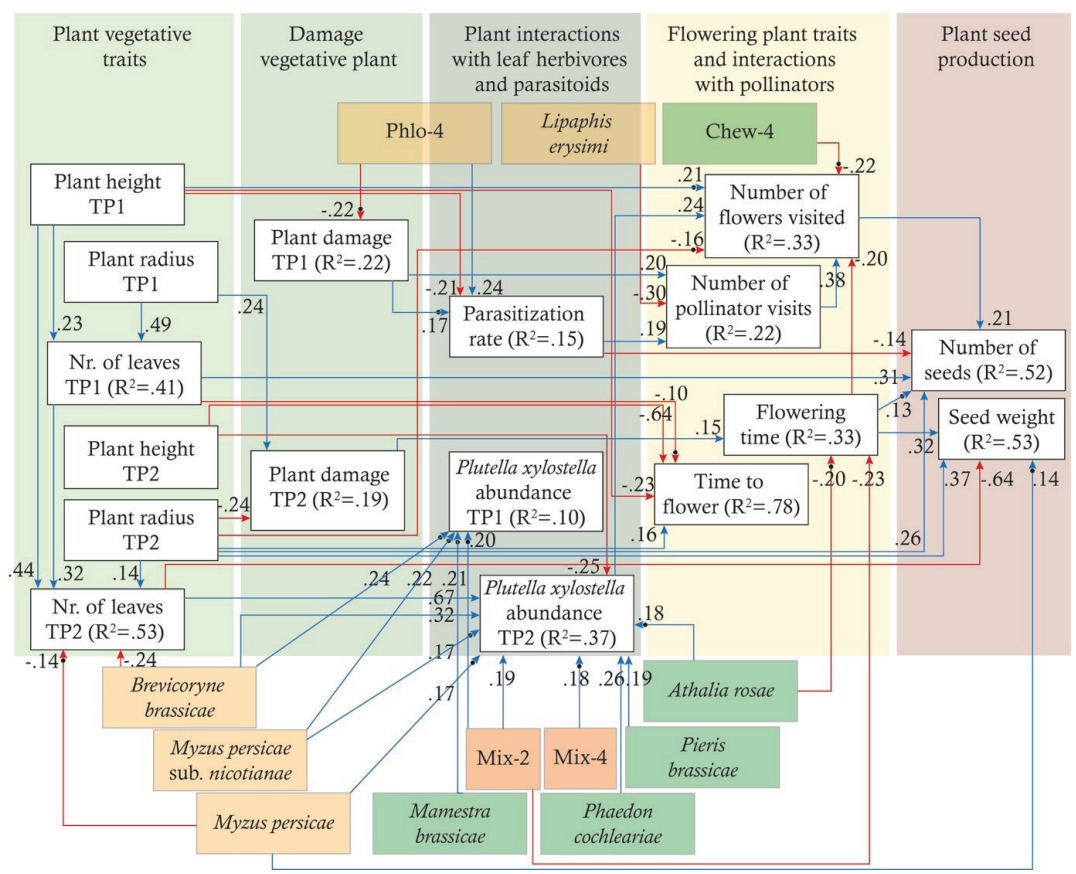

Figure 14. Structural Equation Modelling showing variables directly affected by the herbivore treatments, Blue arrows represent positive links whereas, red negative. A dot in front of the arrows represents correlations where $0.05<P<0.10$. TP1 and TP2 refer to measurements made at the first and second time point, respectively. Chew-4, and Phlo-4 are plants that received four species of chewers and phloem feeders, respectively. Mix-2 and Mix-4 are plants that respectively received four or two species of a mix of chewers and phloem feeders.

\section{DISCUSSION}

Herbivore richness, its combination of functional traits and specific herbivore species affected the abundance of $P$. xylostella, the parasitism rate of $P$. xylostella and pollinator visitation to B. nigra plants. The composition of the initial herbivore community affected plant fitness predominantly through its effects on plant performance such as growth, flowering time, and amount of leaf damage that strongly correlated with number and weight of seeds produced. In addition, species-rich communities of a single feeding guild (either only leaf chewers 
or only phloem feeders) negatively affected plant fitness indirectly through a chain of interactions involving reduced pollinator visitation rates that positively correlated with seed number. In contrast, plants induced with a mix of phloem feeders and leaf chewers, as well as plants induced by individual herbivore species, promoted the presence of $P$. xylostella that corresponded with enhanced flower visitation and increased seed production. These indirect interactions that resulted in positive effects on seed production did in most cases not compensate for the negative effect of herbivores on fitness via effects on plant performance.

Herbivore richness, herbivore functional traits and herbivore identity affected the interaction network of B. nigra. In general, P. xylostella was more abundant on plants that received herbivory early in the season regardless of the richness and the functional traits, compared with control plants. Likewise, in a field study P. xylostella was more abundant on Brassica oleracea plants attacked by Pieris rapae, compared with undamaged plants (Poelman et al., 2008). In our study, not only P. xylostella was affected by herbivory, but also its parasitoids. The parasitism rate of $P$. xylostella larvae was affected by specific herbivores attacking the plant, and by their feeding guild. Plants attacked by a mix of two chewers and two phloem feeders (i.e. Mix-4) had higher rates of parasitism than parasitism rates on control plants. A recent study showed that compared to the performance on undamaged plants, P. xylostella grew less on plants induced by chewers, while on mixed-induced plants the performance was not reduced (Fernández de Bobadilla et al., 2021). Parasitoids may be more attracted to mixedinduced plants because of the higher detectability and the association of the mixed-induced volatiles with a higher quality of the host (Aartsma et al., 2019). Additionally, in a choice assay in the greenhouse, P. xylostella laid fewer eggs on plants attacked by a mix of two chewers and two phloem feeders than on plants attacked by phloem feeders. The reduced preference of P. xylostella towards the plants that in the field received the highest parasitism may be indicative of an adaptation of P. xylostella to avoid herbivore communities that predict high risk of parasitism. 
In our study, P. xylostella abundance did not have direct effects on plant fitness. Thus herbivore-herbivore interactions between the species we studied did not explain fitness. However, P. xylostella abundance connected with an increase in the number of flowers visited by pollinators, which was positively correlated with plant fitness. This suggests that interactions from early-season herbivory to a later arriving herbivore that affects flower visitation by pollinators imposes selection on plant traits (Mertens et al., 2021; Rusman et al. 2019a). Similarly, simulated florivory in Impatiens capensis plants, reduced pollinator and pollen thieve visits, and the alterations in interactions affected plant fitness (Soper Gorden \& Adler, 2016, 2018). Plants attacked by the phloem feeder L. erysimi and by the four chewers simultaneously (i.e. Chew-4) received fewer visits by pollinators. Likewise, a recent study shows that herbivory by aphids caused nectar shortage which reduced pollination visitation and led to a reduction in plant fitness (Devegili \& Chalcoff, 2020). The herbivore-induced effects on plant interactions with herbivores, parasitoids and pollinators could be attributed to a change in important cues for insect foraging, such as the volatile blend or visual cues (Kessler \& Chautá, 2020; Moreira et al., 2019; Rusman et al., 2019b).

Plant fitness was affected by the richness of herbivores attacking the plant, by their feeding guild, and by their interaction. Attack by the highest richness of chewers and phloem feeders (i.e. Chew- 4 and Phlo- 4 respectively) as well as by $A$. rosae, or by $M$. persicae sub. nicotianae negatively affected plant fitness in terms of seed numbers. The negative effect on plant fitness was connected with a reduction in pollinator visitation. Additionally, when feeding individually, the two most common aphids attacking B. nigra (i.e. M. persicae and B. brassicae) (Mertens et al., 2021) had a positive effect on seed weight by inducing a reduction in the number of leaves. The negative correlation between number of leaves and seed production could be explained by the plant reallocating the energy used for leaves to other parts, such as the roots, increasing the plant nutrient uptake (Ruan et al., 2013). Additionally, a reduction in numbers of leaves could make the plant less attractive to herbivores not monitored in this study that have strong fitness consequences. However, we did not find any correlation between 
seed weight and leaf damage. Therefore, the herbivore reducing plant fitness on plants with fewer leaves should be a species that does not cause notable leaf damage to the plant at the time measured (e.g. a root- or phloem-feeding herbivore). Delia radicum larvae feed on Brassicaceae roots, causing high fitness costs being unnoticed for weeks (Soler et al., 2005, 2007). Alternatively, the estimated leaf damage of a full plant might not be a good predictor of plant fitness (de Vries et al. 2017, 2018, 2019). In contrast to the positive effect of some individual phloem feeder species on plant fitness, when phloem feeders attacked the plant in combination with chewers (i.e. Mix) the collective effect on plant fitness was negative. Induction of plants with only leaf chewers also reduced plant fitness. Plants attacked by the chewer $A$. rosae and a by mix of one chewer and one phloem feeder (i.e. Mix-2) produced lighter seeds through reduced flowering time. The reduction in seed weight linked to shorter flowering is potentially explained by a reduction in pollinator visitation or by lower quality pollination of individual flowers.

We show that herbivore richness, functional trait diversity and herbivore identity affect plant fitness and plant interactions with P. xylostella, parasitoids and pollinators. Herbivory strongly affected plant fitness through reducing plant performance and flowering time. Indirect interactions between subsequent herbivores, parasitoids and pollinators affected plant fitness mediated by plant traits. All of these interactions involved effects of inducing herbivores on pollinators that closely connected with the total number of seeds produced by a plant. Under climate change and insect decline, where herbivore communities are predicted to be poorer in species richness, we may expect effects on plant trait evolution. One of the groups that is already suffering a worrying decline is pollinators (Althaus et al., 2021). If the pollinator decline situation continues, pollinator limitation will likely magnify the fitness consequences of indirect interactions observed in our study, as all the routes affecting plant fitness connected to pollinators. Additionally, the natural biological control of herbivores by parasitoids is expected to be disrupted due to climate change (Chidawanyika et al., 2019). Under climate change, predictions pose that plants 
will suffer more stress and the extensive effects of herbivore richness, functional trait and identity on the interaction network will likely add to this impact on plant fitness (Wagner et al., 2021).

Our study has uncovered the tip of the iceberg of how herbivore richness, functional trait and identity alters direct and indirect interactions with other insects and has fitness effects on plants. Our work allows us to predict that the current situation of insect decline and climate change will have dramatic consequences on the structure of the community interacting with a plant. However, due to the complexity and high connectivity of the interactions network it is very difficult to make predictions on directions of effects for plant trait selection.

ACKNOWLEDGEMENTS The authors thank Sean Zending for helping in data collection of the plant flowering traits and pollinator visitation. We also thank Karin Winkler for her kind help with the design of the device used for the parasitism of $P$. xylostella experiment. We thank the staff of Unifarm for setting up and maintaining the experimental fields. 


\section{SUPPLEMENTARY INFORMATION}

\section{Materials and methods from experiments studying the effect of herbivore richness and guild on the oviposition preference of Plutella xylostella on Brassica nigra plants}

\section{Plants and insects}

3-4 weeks old black mustard, Brassica nigra (Brassicales: Brassicaceae) were used for the experiments. The plants were grown and maintained in a greenhouse at $22 \pm 2{ }^{\circ} \mathrm{C}, 60-70 \% \mathrm{RH}$ and 16:8 h L:D photo regime. Insects used as inducers were the same species and reared in the same conditions as the ones used the field experiment (see main text).

\section{Plant induction and Plutella xylostella oviposition preference}

Four leaves of each plant were induced with the herbivore treatments, which were the same treatments that were used in the field study (Con-0, Phlo-1, Phlo-4, Chew-1, Chew-4, Mix-2, Mix-4). Each of the four leaves received the total number of herbivores applied on the whole plant in the field. After herbivore infestation, each leaf was enclosed in a mesh bag, to prevent the movement of the herbivores to different leaves. The induction lasted for five days. Con- 0 plants functioned as control, these plants did not receive any inducing herbivore, but were treated in a similar way as plants receiving herbivores. Plants that received the different treatments were placed in the greenhouse in a randomized block design.

We prepared plastic oviposition cages, which had as base a large Petri dish $(145 / 20 \mathrm{~mm})$ on which plastic sheets $(210 \times 297 \mathrm{~mm})$ were installed to create the walls of the cages (as described in Poelman et al., 2008) (Fig S1). Two $5 \mathrm{ml}$ glass vials were glued to the bottom of the oviposition cages and were filled with water. Right before the oviposition preference assays, one leaf from each of the two plants to test was detached from the plant with scissors, and the leaf petiole was immediately submerged in one of the two vials filled with water, to avoid dehydration, and the herbivores were removed with a brush. To 
avoid differences in oviposition preference caused by leaf age, pairs of leaves of similar age were chosen for the two-choice set-up. The leaves were placed in a way that prevented them from touching each other and were randomly assigned to one of two positions in the plastic cage (Fig S1).

The oviposition preference of the diamondback moth, Plutella xylostella L. (Lepidoptera: Plutellidae) between leaves of plants that received different treatments was studied. Oviposition preference of P. xylostella moths is mostly driven by post alighting gustatory perception of non-volatile glucosinolates, leaf surface waxes, chemosensory stimulation and abiotic factors (Justus \& Mitchell, 1996; Zhang et al., 2012). One female P. xylostella moth was released in each oviposition cage together with one adult male, to ensure mating. The exact age of the moths was unknown, and younger and older butterflies were mixed to randomize our design. Since it is known that P. xylostella prefers to oviposit at dusk, the oviposition preference was measured twenty-four hours after the release of the couples. This was done by carefully inspecting and counting the number of eggs found on each leaf. The number of eggs present on the walls of the oviposition cage was also recorded. The leaves of each choice set-up and the P. xylostella moths were used only once. Each oviposition cage was considered as one replicate.

\section{Oviposition preference comparisons}

To test if the oviposition preference of P. xylostella moths is affected by the herbivore treatments, we conducted two main experiments, performed one after each other in the same greenhouse compartment. The first experiment was designed to evaluate the effect of herbivory and of herbivore guild on the oviposition preference of $P$. xylostella. To test the effect of herbivory, $P$. xylostella moths were allowed to choose oviposition site between a leaf from an undamaged plant (i.e. Con-0) or from a plant induced with the highest richness of phloem feeders, leaf chewers or a mix of both (i.e. Phlo-4, Chew-4 or Mix4). To test the effect of guild of the attacker, the moths were allowed to choose oviposition site between leaves from the highest herbivore richness of phloem 
feeders, chewers or a mix (Table S1). The experiment was divided in four blocks over time, performed one after each other. Each choice was repeated 12 times in each of the blocks, making a total of 48 replicates per treatment comparison.

In a second experiment we studied the effect of herbivore richness within feeding guild on the oviposition preference of $P$. xylostella. The moths were allowed to choose between leaves induced with the lowest or highest richness of herbivores within each guild (Table S2). This experiment was also divided in four blocks over time, performed one after each other. For the effect of phloem-feeder and chewer richness, each combination was repeated eight times in each of the blocks, making a total of 32 replicates per treatment comparison. For the mixed richness, two species of Mix-2 were compared with the same two species of Mix-2 plus two more species. We selected treatments, so that each combination of one phloem feeder and one chewer species of Mix-2 was tested against three different combinations of Mix-4, making a total of 48 different comparisons (Table S2). Due to the high number of choice combinations, and space and time limitations, each comparison was repeated only once in each of the four blocks.

\section{Statistical analysis}

In some cases, the moths did not lay any egg and these replicates were not included in the analysis (Table S3). In other cases, P. xylostella laid most eggs on the walls of the oviposition cage (Table S3). When the percentage of eggs found on the walls of the cage exceeded $50 \%$, we excluded the replicate from further analysis (Table S3). Oviposition preference data was analysed using twosided paired t-tests if normally distributed, or by Wilcoxon signed-rank tests in case the data did not follow a normal distribution. The normality was visually examined by making histograms of the difference of the eggs that were laid in the $1^{\text {st }}$ choice leaf and the $2^{\text {nd }}$ choice leaf in each dual oviposition preference assay (in each oviposition cage). All the statistical analyses were performed using RStudio version 1.1.463. 
Results from experiments studying the effect of herbivore richness and guild on the oviposition preference of Plutella xylostella on Brassica nigra plants

The oviposition preference of P. xylostella was not affected by herbivory. The preference of P. xylostella did not differ between undamaged leaves and leaves that were induced with the maximum richness of phloem feeders, chewers and a mix of both (Con- 0 vs Phlo-4: $\mathrm{P}=0.1935$; Con- 0 vs Chew- 4 : $\mathrm{P}$ $=0.8736$; paired t-test; Con-0 vs Mix-4: $\mathrm{P}=0.1842$; Wilcoxon signed rank test Fig. S2A-C)). P. xylostella female moths also did not discriminate between leaves induced with highest richness of phloem feeders and chewers, nor between leaves induced with the highest richness of chewers and mix (Phlo-4 vs Chew-4: $\mathrm{P}=0.87$, paired t-test; Chew-4 vs Mix-4: $\mathrm{P}=0.34$; Wilcoxon signed rank test; Fig. S2 D, E). In contrast, the moths preferred to lay eggs on leaves induced with the highest richness of phloem feeders compared to leaves induced with the highest richness of the mix (Phlo-4 - Mix-4: $\mathrm{P}<0.001$; Wilcoxon signed rank test, Fig. S2F).

The richness of phloem feeders did not affect the oviposition preference of P. xylostella and the number of eggs laid by the moths on leaves induced by one species or by four species of phloem feeders was not different (Phlo-1 vs Phlo4: $\mathrm{P}=0.57$, paired t-test; Fig. S3). No significant differences were found in the oviposition preference on leaves induced with Brevicoryne brassicae, Lipaphis erysimi, Myzus persicae and Myzus persicae sub. nicotianae compared with leaves induced with all phloem-feeding species simultaneously (B. brassicae vs Phlo-4: Wilcoxon signed rank test $\mathrm{P}=0.72$, L. erysismi vs Phlo-4: Wilcoxon signed rank test $\mathrm{P}=0.22 ; M$. persicae vs Phlo-4: Wilcoxon signed rank test $\mathrm{P}=0.17 ; M$. persicae sub. nicotianae vs Phlo-4: paired t-test $\mathrm{P}=0.10$; Fig. S3A-D). In line with the lack of phloemfeeder richness on the oviposition preference of P. xylostella, the moths did not discriminate between leaves induced with one or with four chewer species (Chew1 - Chew-4: $\mathrm{P}=0.72$, paired t-test). However, some specific herbivores affected P. xylostella preference. Moths laid more eggs on Phaedon cocbleariae- induced leaves 
than on the maximum chewer diversity (P. cochleariae vs Chew-4: Wilcoxon signed rank test $\mathrm{P}<0.05$; Fig. S4C), while they preferred to oviposit on leaves induced by the highest chewer richness than on Mamestra brassicae-induced leaves ( $M$. brassicae vs Chew-4: Wilcoxon signed rank test P < 0.01; Fig. S4B). In contrast, P. xylostella did not discriminate between leaves induced with the highest richness of chewers and Pieris brassicae-induced or Athalia rosae-induced leaves (P. brassicae - Chew-4: paired t-test $\mathrm{P}=0.46 ; A$. rosae - Chew-4: Wilcoxon signed rank test $\mathrm{P}=0.54$; Fig. S4 A,D). The oviposition preference of P. xylostella was also not affected by the richness of herbivores on a mix of phloem feeders and chewers (Mix-2 vs Mix-4: paired t-test $\mathrm{P}=0.58$; Fig. S5).

\section{Figures from experiments studying the effect of herbivore richness and guild on the oviposition preference of Plutella xylostella on Brassica nigra plants}

(A)

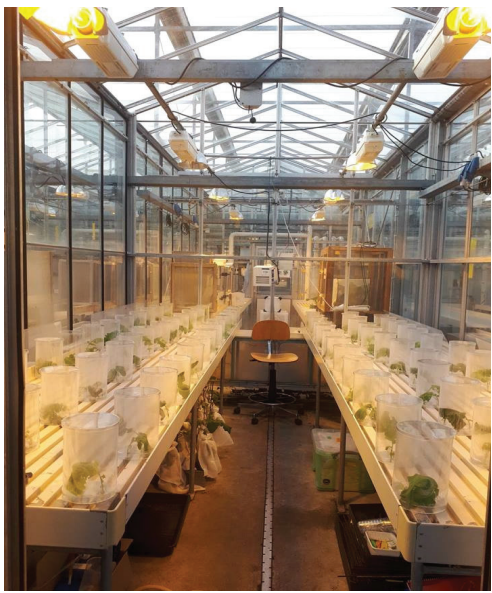

(B)

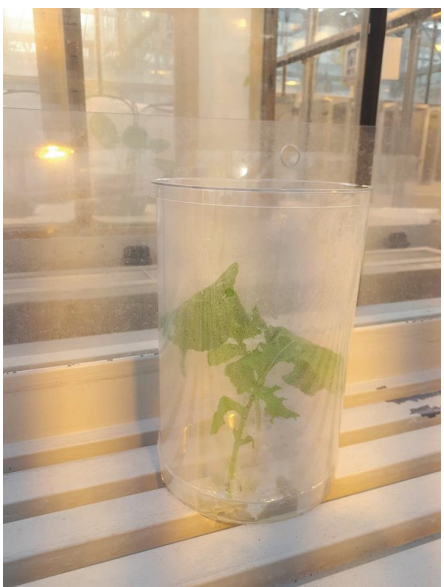

Figure S1: (A) Greenhouse compartment with the cages in which we tested the oviposition preference of Plutella xylostella in one block. (B) Closer look of one oviposition cage, with two leaves coming from two different induction treatments with their petioles in the two glass vials. 

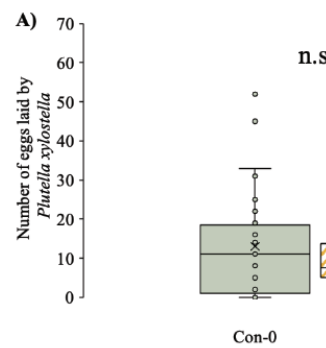

n.s.

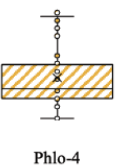

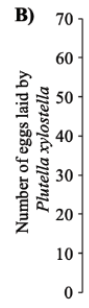

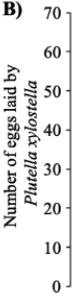

n.s.

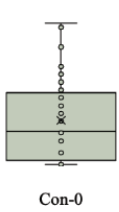

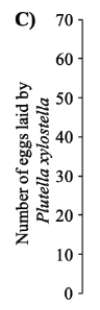

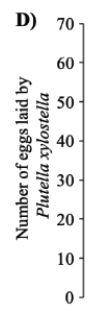

n.s.

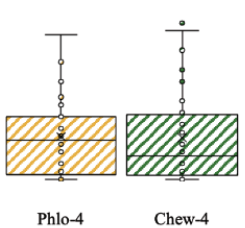

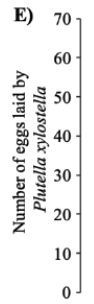

n.s.

F)

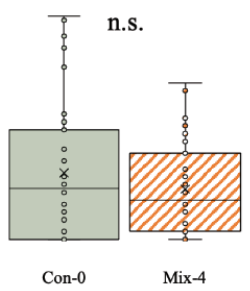

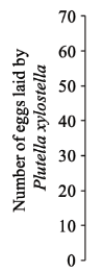

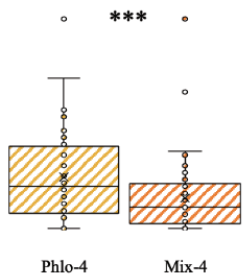

Figure S2. Number of eggs laid by Plutella xylostella moths on each Brassica nigra leaf of the two-choice assay between A) Untreated plants (Con-0) and plants treated with the highest species richness of phloem feeders (Phlo-4). B) Untreated plants (Con-0) and plants treated with the highest species richness of chewers (Chew-4). C) Untreated plants (Con0 ) and plants treated with the highest species richness of a mix of phloem feeders and chewers (Mix-4). D) Plants treated with the highest species richness of phloem feeders (Phlo-4) and plants treated with the highest species richness of chewers (Chew-4). E) Plants treated with the highest species richness of chewers (Chew-4) and plants treated with the highest species richness of a mix of phloem feeders and chewers (Mix-4). F) Plants treated with the highest species richness of phloem feeders (Phlo-4) and plants treated with the highest species richness of a mix of phloem feeders and chewers (Mix-4). Boxplot height corresponds to the first and third quartiles (Q1 and Q3), and the middle line to the median. Asterisks above the boxplots show significant differences ( $* * * P<0.001$; Wilcoxon signed rank test).n.s.=non-significant differences ( $P>0.05$; Wilcoxon signed rank test). 
A)

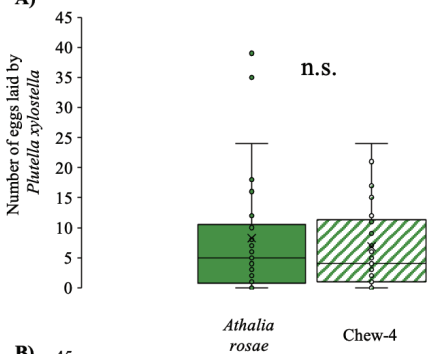

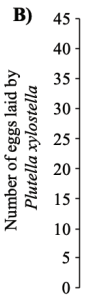

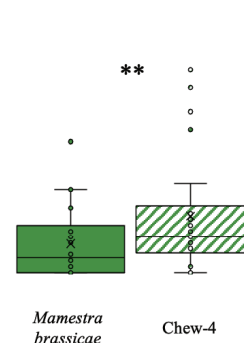

C)

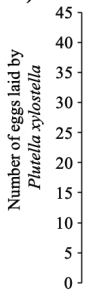

D)

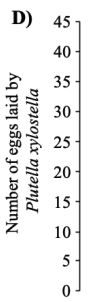

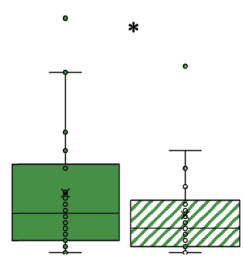

Phaedon cochleariae

Chew-4

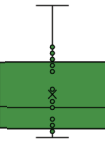

n.s.

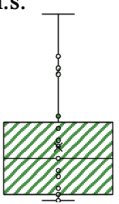

Pieris brassicae

Chew-4

Figure S3. Number of eggs laid by Plutella xylostella moths on each Brassica nigra leaf of the two-choice assay between A) Plants treated with the chewer Athalia rosae and plants treated with the highest species richness of chewers (Chew-4). B) Plants treated with the chewer Mamestra brassicae and plants treated with the highest species richness of chewers (Chew-4). C) Plants treated with the chewer Phaedon cochleariae and plants treated with the highest species richness of chewers (Chew-4). D) Plants treated with the chewer Pieris brassicae and plants treated with the highest species richness of chewers (Chew-4). Boxplot height corresponds to the first and third quartiles (Q1 and Q3), and the middle line to the median. Asterisks above the boxplots show significant differences ( $\mathrm{P}$ $<0.05$; $* * \mathrm{P}<0.01$; Wilcoxon signed rank test). n.s.=non-significant differences $(\mathrm{P}>0.05$; Wilcoxon signed rank test). 


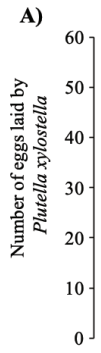

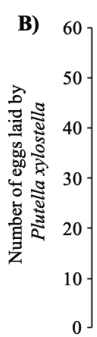

n.s.

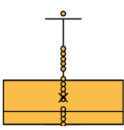

Brevicoryne brassicae

n.s.

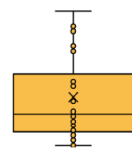

Lipaphis erysismi
C)

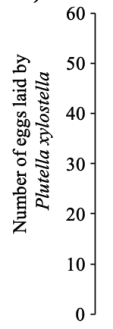

n.s.

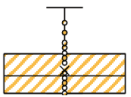

Phlo-4

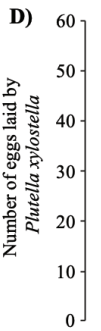

Myzus persicae

Phlo-4

n.s.
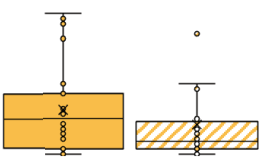

Myzus

Persicae sub. nicotianae

Phlo-4

Figure S4. Number of eggs laid by Plutella xylostella moths on each Brassica nigra leaf of the two-choice assay between A) Plants treated with the phloem feeder Brevicoryne brassicae and plants treated with the highest species richness of phloem feeders (Phlo-4). B) Plants treated with the phloem feeder Lipaphis erysismi and plants treated with the highest species richness of phloem feeders (Phlo-4). C) Plants treated with the phloem feeder Myzus persicae and plants treated with the highest species richness of phloem feeders (Phlo-4). D) Plants treated with the phloem feeder M. persicae sub. nicotianae and plants treated with the highest species richness of phloem feeders (Phlo-4). Boxplot height corresponds to the first and third quartiles (Q1 and Q3), and the middle line to the median. n.s.=non-significant differences $(P>0.05$; Wilcoxon signed rank test). 


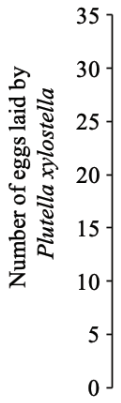

n.s.

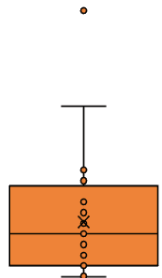

Mix-2

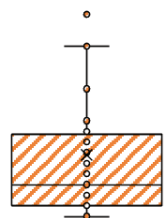

Mix-4

Figure S5. Number of eggs laid by Plutella xylostella moths on each Brassica nigra leaf of the two-choice assay between plants treated with the two or with four species of a mix of phloem feeders and chewers (Mix-2 or Mix-4). Boxplot height corresponds to the first and third quartiles (Q1 and Q3), and the middle line to the median. Asterisks above the boxplots show significant differences. n.s.=non-significant differences $(P>0.05$; Wilcoxon signed rank test).

Table S1. Herbivore combinations included in the two-choice oviposition experiment 1 . The experiment was divided in four blocks over time. Each comparison was repeated 12 times per block, making a total of 48 replicates per choice.

\begin{tabular}{cc}
\hline Leaf 1 & Leaf 2 \\
\hline Con-0 & Phlo-4 \\
\hline Con-0 & Chew-4 \\
\hline Con-0 & Mix-4 \\
\hline Phlo-4 & Chew-4 \\
\hline Phlo-4 & Mix-4 \\
\hline Chew-4 & Mix-4 \\
\hline
\end{tabular}


Table S2. Herbivore combinations included in the two-choice oviposition experiment 2. The experiment was divided in four blocks over time. The phloem feeder and chewer richness comparisons were repeated eight times per block, making a total of 32 replicates per choice. Each of the 48 Mix-2 Mix-4 comparisons was repeated only once. Phloem feeding aphids Brevicoryne brassicae (Bb), Lipaphis erysimi (Le), Myzus persicae (Mp), Myzus persicae sub. nicotianae (Mpn). Chewers: Athalia rosae (Ar), Mamestra brassicae (Mb), Phaedon cochleariae $(P C)$, Pieris brassicae $(P b)$.

\begin{tabular}{|c|c|}
\hline Leaf 1 & Leaf 2 \\
\hline Phlo-1 & Phlo-4 \\
\hline$B b$ & \multirow{4}{*}{$B b+M p+M p n+L \epsilon$} \\
\hline$M p$ & \\
\hline Mpn & \\
\hline Le & \\
\hline Chew-1 & Chew-4 \\
\hline$A r$ & \multirow{4}{*}{$A r+P b+P b+M b$} \\
\hline $\mathrm{Pb}$ & \\
\hline$P C$ & \\
\hline$M b$ & \\
\hline Mix-2 & Mix-4 \\
\hline$B b M b$ & \multirow{4}{*}{$B b M p M b A r$} \\
\hline$B b A r$ & \\
\hline$M p M b$ & \\
\hline$M p A r$ & \\
\hline$B b P b$ & \multirow{4}{*}{$B b M p P b P c$} \\
\hline$B b P c$ & \\
\hline$M p P b$ & \\
\hline$M p P c$ & \\
\hline$B b M b$ & \multirow{4}{*}{$B b M p n M b P c$} \\
\hline$B b P c$ & \\
\hline MpnMb & \\
\hline$M p n P c$ & \\
\hline
\end{tabular}


Table S2. Continuation.

\begin{tabular}{|c|c|}
\hline Leaf 1 & Leaf 2 \\
\hline$B b A r$ & \multirow{4}{*}{$B b M p n A r P b$} \\
\hline$B b P b$ & \\
\hline$M p n P b$ & \\
\hline MpnAr & \\
\hline$B b M b$ & \multirow{4}{*}{$B b L e M b P b$} \\
\hline$B b P b$ & \\
\hline LeMb & \\
\hline$L e P b$ & \\
\hline$B b A r$ & \multirow{4}{*}{$B b L e$ ArPc } \\
\hline$B b P c$ & \\
\hline LeAr & \\
\hline LePC & \\
\hline$M p M b$ & \multirow{4}{*}{$M p M p n M b P b$} \\
\hline$M p P b$ & \\
\hline MpnMb & \\
\hline$M p n P b$ & \\
\hline$M p A r$ & \multirow{4}{*}{$M p M p n \operatorname{ArPc}$} \\
\hline$M p P c$ & \\
\hline MpnAr & \\
\hline$M p n P c$ & \\
\hline$M p M b$ & \multirow{4}{*}{ MpLe MbPc } \\
\hline$M p P c$ & \\
\hline LeMb & \\
\hline LePC & \\
\hline
\end{tabular}


Table S3. Overview of replicates included for the oviposition experiments. "No response" indicates the number of set-ups where the moths did not lay any egg. The "No choice" indicates the set-ups where the moths laid more than $50 \%$ of the eggs on the oviposition cages instead than on the two choice leaves. The "No response" and "No choice" columns were excluded from the data analysis. Phloem feeding aphids $B b=$ Brevicoryne brassicae, $L e=$ Lipaphis erysismi, Mp= Myzus persicae, Mpn= Myzus persicae sub. nicotianae, Ar= Athalia rosae, $\mathrm{Mb}=$ Mamestra brassicae, $P b=$ Pieris brassicae, $P c=$ Phaedon cochleariae.

\begin{tabular}{ccccc}
\hline Comparisons & Replicates tested & "No response" & "No choice" & $\begin{array}{c}\text { Replicates included } \\
\text { in the analysis }\end{array}$ \\
\hline Con-0 vs Phlo-4 & 48 & 9 & 0 & 39 \\
\hline Con-0 vs Chew-4 & 48 & 6 & 1 & 41 \\
\hline Con-0 vs Mix-4 & 48 & 10 & 0 & 38 \\
\hline Phlo-4 vs Chew-4 & 48 & 7 & 1 & 40 \\
\hline Phlo-4 vs Mix-4 & 48 & 7 & 2 & 39 \\
\hline Chew-4 vs Mix-4 & 48 & 14 & 1 & 33 \\
\hline Phlo-1 vs Phlo-4/Total & 128 & 26 & 3 & 99 \\
\hline Bb vs Phlo-4 & 32 & 6 & 0 & 26 \\
\hline Le vs Phlo-4 & 32 & 6 & 0 & 26 \\
\hline Mp vs Phlo-4 & 32 & 6 & 3 & 24 \\
\hline Mpn vs Phlo-4 & 32 & 8 & 0 & 87 \\
\hline Chew-1- Chew-4/Total & 128 & 36 & 5 & 24 \\
\hline Ar vs Chew-4 & 32 & 6 & 2 & 20 \\
\hline Mb vs Chew-4 & 32 & 10 & 2 & 22 \\
\hline$P b$ vs Chew-4 & 32 & 11 & 0 & 28 \\
\hline$P c$ vs Chew-4 & 32 & 9 & 1 & 0 \\
\hline Mix-2 vs Mix-4 & 48 & 20 & & 22 \\
\hline
\end{tabular}




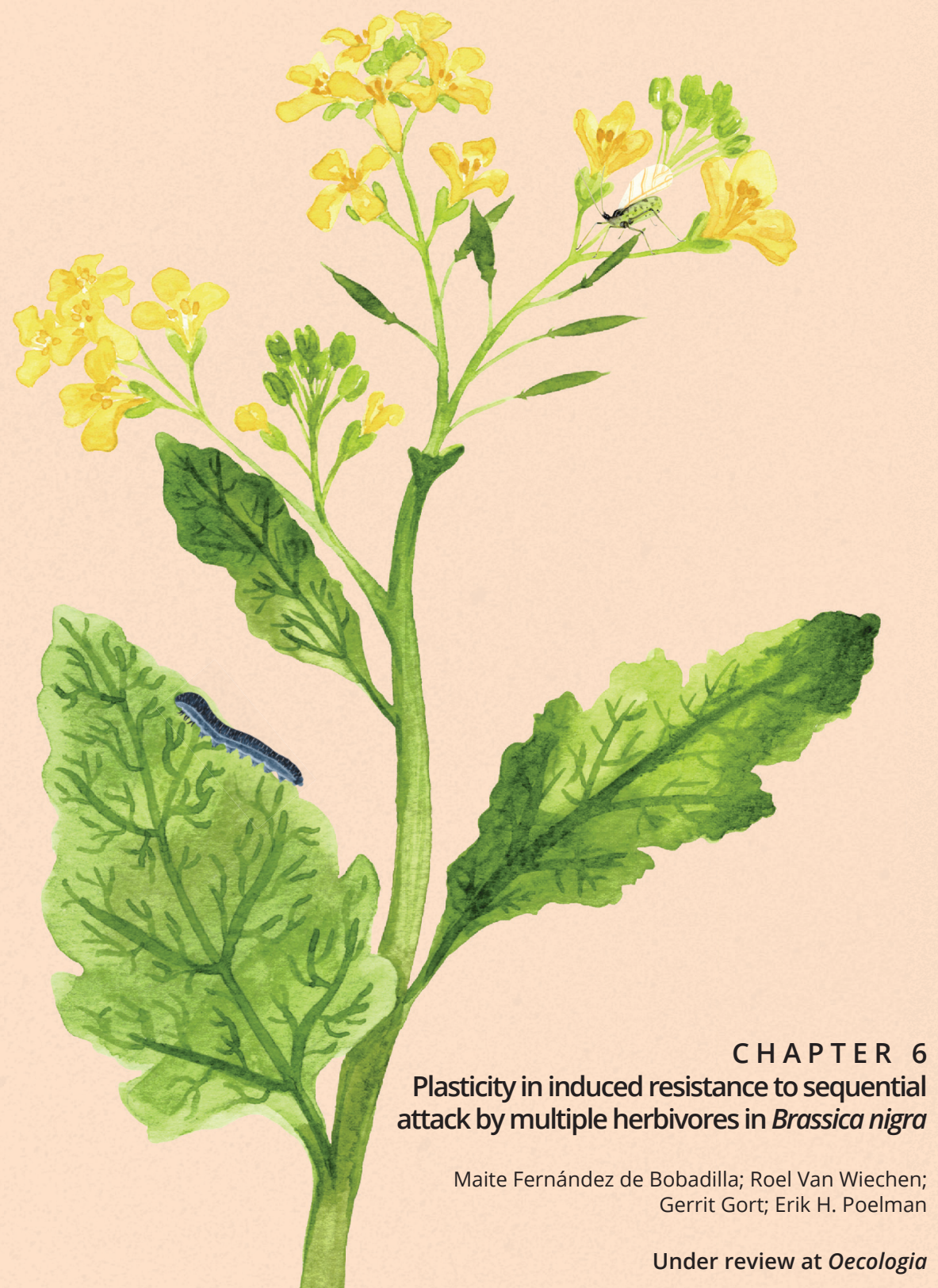





\section{ABSTRACT}

In nature, plants interact with multiple insect herbivores that may arrive simultaneously or sequentially. Because insect herbivores often have a negative effect on plant fitness, plants have evolved mechanisms to defend their tissues from being eaten. There is extensive knowledge on the regulation of induced plant responses to single or dual attack. However, we lack information on how plants defend against the attack of multiple herbivores that arrive sequentially. In this study, we investigated whether Brassica nigra plants are able to defend themselves against caterpillars of the late arriving herbivore Plutella xylostella, when plants had been previously exposed to sequential attack by four other herbivores. We manipulated the order of arrival and the history of attack by four herbivores to investigate which patterns in sequential herbivory determine resistance against the fifth attacker. We recorded that history of sequential herbivore attack differentially affected the capability of Brassica nigra plants to defend themselves against larvae of P. xylostella. A sequence of attack with four episodes of attack by P. xylostella induced resistance to larvae of P. xylostella. The number of times the plant was attacked by herbivores of the same feeding guild, the identity of the first attacker, the identity and the guild of the last attacker as well as the order of attackers within the sequence of multiple herbivores influenced plant resistance to subsequent herbivory. In conclusion, this study shows that history of sequential attack is an important factor determining plant resistance to herbivores. 


\section{INTRODUCTION}

In nature, plants interact with a species-rich community of insects (Giron et al., 2018). Some interactions with insects are beneficial for the plant, such as those with pollinators, whereas others are detrimental such as interactions with herbivores. Plants are exposed to the attack of multiple insect herbivores that feed from their tissues simultaneously or sequentially. Because insect herbivores often have a negative effect on plant fitness, plants have evolved defence mechanisms to prevent herbivores from eating their tissues (Erb, 2018). Some of those defence mechanisms are constitutive, and are always expressed independently of the presence of the attacker (War et al., 2012). However, maintaining defences is metabolically costly, and herbivores are very diverse in the way they consume the plant and in the defence traits they are sensitive to. Therefore, plants have evolved induced defences, that are only expressed upon attack and are more specific to the attacker compared to constitutive defence (Karban, 2011; Karban et al., 1997).

Studies on plants under single attack show specificity in the phytohormonal regulation of induced plant defence (Erb et al., 2012). The phytohormonal pathways that regulate induced plant responses cross-communicate with either positive, negative or neutral interactions between pathways (Pieterse et al., 2012; Soler et al., 2012; Thaler et al., 2012). The main phytohormones regulating plant responses to insects are jasmonic acid (JA) and salicylic acid (SA), but other phytohormones such as ethylene (ET) or abscisic acid (ABA) also play a role (Erb et al., 2012; Erb \& Reymond, 2019). In general, plant responses to leaf-chewing insects are regulated via the JA pathway, whereas plant responses to phloemfeeding insects are regulated via the SA pathway (Erb \& Reymond, 2019). JA and SA often have a negative crosstalk (Moreira et al., 2018). The crosstalk between phytohormones may compromise plant defences facing sequential attack by two insects of a different guild, where depending on the identity or guild of the first attacker the plant may become more resistant or more vulnerable to the attack of the second. Phytohormonal crosstalk between the SA and JA pathways often impairs plant responses to chewing herbivores when the plant has already 
directed its resistance response towards a phloem-feeding herbivore (Moreira et al., 2018; Soler et al., 2012). However, a recent study on dual attack testing 90 pairwise interactions between Brassica nigra plants and 10 herbivores shows that plants are not always limited to respond to chewers when they have been previously attacked by phloem feeders. Interestingly $B$. nigra plants adapt their induced response to the first attacker depending on the likelihood of subsequent attack determined by the prevalence of a second attacker in the field (Mertens, et al., 2021a,b). This study challenges the so-called JA-SA negative crosstalk paradigm, showing that to truly understand plant defence strategies to multiple herbivores many aspects still need to be explored.

One of the aspects of plant defence strategies that deserves more attention is how plants deal with the common situation of multi-herbivore attack. Only a few studies have explored plant defences to sequential herbivory beyond dual attack. These studies show that the order, the identity and the species richness of the attackers influences plant defence against a late arriving herbivore (Fernández de Bobadilla et al., 2021; Mathur et al., 2013; Stam et al., 2014, 2017, 2018, 2019). Nonetheless, in nature, herbivores often arrive sequentially, and studies including a realistic number of sequential attackers are lacking. This largely limits our understanding of how plastic plants adapt their defence phenotype to attackers that arrive in sequences. Several factors may determine the plant's ability to deal with sequential herbivore attack, such as the frequency of exposure to an attacker of a different feeding guild or the order of arrival of the attackers in the sequence such as the guild/identity of the first or last attacker in the sequence (Erb et al., 2011). Plants need to maximize their defence response to a current attacker in the context of an optimal response to the dynamic community of herbivores that may arrive later and also affect plant fitness (Mertens, et al., 2021; Poelman \& Kessler, 2016).

The aim of this study was to investigate whether plants are able to defend themselves against a late arriving leaf chewing herbivore, when they had been previously exposed to sequential attack by four other herbivores. We 
subjected black mustard (B. nigra) plants to attack by 12 different sequences of herbivory or left the plants without herbivory and investigated plant defence against caterpillars of Plutella xylostella. We manipulated the number of times there were leaf-chewing or phloem-feeding insects in the four incidences of herbivore attack, as well as the order of the attackers and their species identity. We hypothesized that plants attacked more frequently by a leaf chewer would have a stronger JA induction and therefore would be better prepared to respond against a late arriving leaf chewer. Because the response to a first herbivore may most profoundly determine the capabilities of plants to deal with subsequent attack (Viswanathan et al., 2007), plants first attacked by a chewer may be better able to respond against a late arriving chewer compared to plants first attacked by a phloem feeding herbivore. Similarly, plants more recently attacked by a chewer may show a stronger induced defence towards chewers, compared with plants more recently attacked by a phloem feeder. Thus, we hypothesized that when the most recent attacker (i.e. the last in a sequence of four) was a chewer, plants would be more resistant to a late arriving chewer (fifth herbivore) than plants that had a phloem feeder as most recent attacker. Finally, we hypothesized that the order of the herbivores in the sequence would be an important factor in determining plant resistance against a late arriving herbivore.

\section{MATERIALS AND METHODS \\ Plants and insects}

Two and a half weeks old black mustard plants (B. nigra, Brassicales: Brassicaceae) were used for the experiments. Seeds were obtained from a natural population in the vicinity of Wageningen ( $\left.51^{\circ} 57^{\prime} 32^{\prime \prime} \mathrm{N}, 5^{\circ} 40^{\prime} 23^{\prime \prime} \mathrm{E}\right)$. The plants and the insects were grown and maintained in a greenhouse at $22 \pm 2{ }^{\circ} \mathrm{C}$, 60-70 \% RH and 16:8 h L:D photo regime. Under natural conditions, individual B. nigra plants, a common annual in the Netherlands, are attacked by at least four up to twelve different herbivore species over their life-time (Mertens, et al., 2021b; Poelman et al., 2009). We used four herbivore species to simulate sequential attack: second instar larvae of the diamondback moth Plutella xylostella $(P x)$ (Lepidoptera: Plutellidae) and first instar larvae the turnip sawfly, Athalia 
rosae ( $A r)$ (Hymenoptera: Tenthredinidae) as leaf chewers and the cabbage aphid, Brevicoryne brassicae $(B b)$ and the green peach aphid, Myzus persicae (Mp) (both Hemiptera: Aphididae) as phloem feeders (Table 1). The degree of resistance induced by sequential attack by different sequences of attack by four herbivores was determined by assessing the performance of second instar larvae of the diamondback moth P. xylostella (Lepidoptera: Plutellidae) as fifth herbivore (that we call receiver). Larvae of this insect are specialists on brassicaceous plants and feed on foliar tissue, buds and flowers. Plutella xylostella typically arrives later in the vegetative growing season of B. nigra plants and often has to deal with plants previously damaged by other herbivores (Mertens et al., 2021b). Brevicoryne brassicae and P. xylostella were reared on Brussels sprouts (Brassica oleracea L. var. gemmifera cv. Cyrus). Myzus persicae and $A$. rosae were reared on radish (Raphanus sativus). All insects were obtained from the stock rearing of the Laboratory of Entomology, Wageningen University.

Table 1. Insects used for the experiment, in brackets abbreviations used throughout the document.

\begin{tabular}{|c|c|c|c|}
\hline Species (Abbreviation) & Picture & Order & Feeding guild \\
\hline Athalia rosae $(\mathrm{Ar})$ & & Hymenoptera & Leaf chewer \\
Plutella xylostella $(\mathrm{Px})$ & & Lepidoptera & Leaf chewer \\
Brevicoryne brassicae $(\mathrm{Bb})$ & & Hemiptera & Phloem feeder \\
Myzus persicae $(\mathrm{Mp})$ & & Hemiptera & Phloem feeder \\
\hline
\end{tabular}

\section{Assessing plant resistance induced by a history of sequential attack by four herbivores}

To test plant plasticity to multiple herbivore attack, we challenged $B$. nigra plants with several herbivore sequences and assessed whether responses to the herbivore sequences differentially affected induced plant resistance to subsequent herbivory by $P$. xylostella as reflected in the performance of caterpillars feeding on induced plants (Figure 1). 


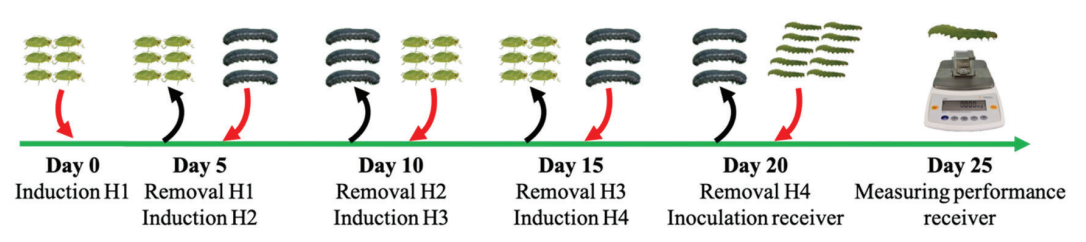

Figure 1. Experimental timeline depicting the herbivore sequences. On Day 0 each Brassica nigra plant was induced with the first set of herbivores $(\mathrm{H} 1)$ which consisted of either three chewers or six aphids. After five days all the herbivores were removed from the plant using a brush, and the second set was introduced $(\mathrm{H} 2)$. This was done for four events of an herbivore sequence. After the herbivores of the last round of herbivory $(\mathrm{H} 4)$ had been removed from the plant, 10 larvae of Plutella xylostella were introduced on each plant and they were allowed to feed from the induced plants for five days. Each $P$. xylostella larva was recaptured and weighed as a measure as of plant resistance.

We prepared a total of 18 plant replicates per herbivore combination divided in two blocks separated in time (nine plant replicates per herbivore combination in each block). We assessed induced plant responses to sequential attack by four herbivores, with a total of 12 treatments that differed in the order and identity of herbivore attack. For each of the four episodes of herbivory in a sequence, the total number used was three leaf chewers or six phloem feeders. The number of individuals per species initially introduced is representative for natural herbivore communities on B. nigra plants (Mertens et al., 2021b). We also prepared control plants that did not receive any inducing herbivore, but were otherwise treated in a similar way as plants receiving herbivores. Each herbivore episode in the sequence lasted for a period of five days, in which the herbivores were allowed to freely feed from the plant. After these five days all the inducers were removed with a fine brush, to exclude direct effects of inducing herbivores on the next herbivore in the sequence and on the receiving herbivore. By removing herbivores, we could focus on distinct events of herbivory, without aphid populations or chewing herbivores overexploiting plants due to the absence of control by their natural enemies. After plants had been exposed to their treatment of sequential 
attack by a sequence of four herbivore species and these herbivores had been removed, each plant was infested with 10 second instar larvae of P. xylostella, that acted as receiver to assess whether induced resistance to this herbivore was differentially affected by the history of herbivore attack (Figure 1). The mass of P. xylostella caterpillars was measured after five days of feeding on the induced plants as a proxy of plant resistance $\left(19^{\circ} \mathrm{C}, 60-70 \% \mathrm{RH}\right.$ and 16:8 $\mathrm{h}$ L:D photo regime). This was done by recapturing the P. xylostella larvae and weighing each individual on a Sartorius ${ }^{\circledR}$ - CP2P - Analytical Balance (accuracy $0.001 \mathrm{mg}$ ).

Table 2. List of herbivore sequences $(\mathrm{H})$ applied to Brassica nigra plants to assess plant resistance to Plutella xylostella. Leaf chewers: $P x=P$. xylostella, $A r=$ Athalia rosae. Aphids: $B b=$ Brevicoryne brassicae, $M p=$ Myzus persicae.

\begin{tabular}{|c|c|c|c|c|}
\hline Treatment & H1 & H2 & H3 & H4 \\
\hline Ctrl & - & - & - & - \\
\hline$P x-P x-P x-P x$ & Drane & Anom & Amomen & minn \\
\hline$A r-A r-A r-A r$ & 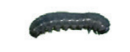 & 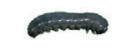 & 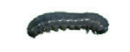 & 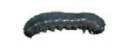 \\
\hline$B b-B b-B b-B b$ & ( & 苚 & त) & ( \\
\hline$M p-M p-M p-M p$ & $\Leftrightarrow$ & $\Rightarrow$ & 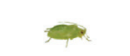 & $\Leftrightarrow$ \\
\hline$B b-B b-P x-P x$ & n & ( & Dinnen & Drim \\
\hline$B b-P x-B b-P x$ & is & Iming & (A & Then \\
\hline$M p-M p-A r-A r$ & 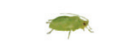 & $\Rightarrow$ & 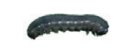 & 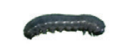 \\
\hline$M p-A r-M p-A r$ & $\infty$ & $\leadsto$ & 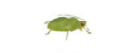 & $\Longrightarrow$ \\
\hline$P x-P x-B b-B b$ & Anom & Anom & (n) & (4) \\
\hline$P x-B b-P x-B b$ & monsm & (n) & 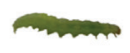 & 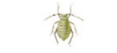 \\
\hline$A r-A r-M p-M p$ & $\Leftrightarrow$ & 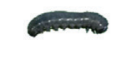 & $\theta$ & $\Rightarrow$ \\
\hline$A r-M p-A r-M p$ & 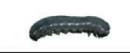 & $\Rightarrow$ & 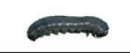 & $\Rightarrow$ \\
\hline
\end{tabular}




\section{Statistical analysis}

To investigate whether the history of herbivory on B. nigra affected the growth of larvae of P. xylostella, we performed several analyses. For all tests we fitted a Mixed Linear Model (MLM), using as fixed effects the time blocks (with two levels) and appropriate part of the data grouped in order to answer the question of interest. Plants were included as random factors and residual error was also included. First, to assess whether the herbivore sequences influenced the plant's capability to defend against larvae of P. xylostella feeding as fifth herbivore, we used fixed effects for all the treatments (with 13 levels: 12 herbivore sequences and a control). Second, to compare the treatments that received four times the same herbivores, we used fixed effects for the selected treatments (with five levels: control, and four sequences of either P. xylostella, A. rosae, B. brassicae or M. persicae). Third, to explore whether the number of times the plant was attacked by an insect of the same feeding guild affected its capability to defend against $P$. xylostella, we grouped our data based on the number of switches of chewers or of aphids in the four episodes of sequential herbivory, using this time fixed effects for number of sequences of chewers/aphids (with four levels: control, zero, two or four). For the rest of the analyses, we excluded the data from the treatments that received four times the same herbivore. We did so because we were interested in comparing the effect of herbivore sequences that contained an equal number of switches of attackers in the sequence. First, we used fixed effects for guild of the first or of the last attacker (with three levels: control, chewer or aphid). Then, we used fixed effects for species identity (with five levels: control, P. xylostella, A. rosae, B. brassicae and M. persicae). Finally, we tested for the effect of order of herbivores within the sequence, and compared treatments that received the same herbivores but in different order.

\section{RESULTS}

\section{History of sequential herbivore attack affects resistance against larvae of P. xylostella}

The sequence of herbivore attack affected the capability of $B$. nigra plants to defend against larvae of P. xylostella $\left(\mathrm{F}_{1,12}=3.42, \mathrm{P}<0.001\right.$, Figure 2$)$. A sequence of attack by four episodes of leaf-chewing caterpillars of $P$. xylostella 
induced resistance to larvae of P. xylostella that were feeding on these plants as fifth herbivore $\left(\mathrm{t}_{1,2}=-2.17, \mathrm{P}=0.032\right.$, Figure 2$)$. No resistance was induced by a sequence of four episodes of attack by the other leaf chewer $A$. rosae. Performance of $P$. xylostella caterpillars feeding on plants induced by a history of four rounds of A. rosae did not differ from $P$. xylostella caterpillars feeding on plants that did not receive herbivory. Sequential attack by four rounds of aphid attack by either $M$. persicae or B. brassicae did not affect the performance of P. xylostella caterpillars as compared to performance on undamaged plants. However, specific sequences of herbivore attack affected the performance of $P$. xylostella caterpillars compared to performance on undamaged plants. Plants became more resistant to P. xylostella larvae after plants had been exposed to sequential attack by $M$. persicae $-M$. persicae $-A$. rosae $-A$. rosae $\left(\mathrm{t}_{1,2}=-4.42, \mathrm{P}<0.001\right.$, Figure 2$)$ and by $M$. persicae $-A$. rosae $-M$. persicae $-A$. rosae $\left(\mathrm{t}_{1,2}=-2.34, \mathrm{P}=0.020\right.$, Figure 2$)$.

\section{Importance of specific events in sequential attack for resistance to $P$. xylostella caterpillars}

The differential effect of specific orders of sequential herbivore attack on performance of $P$. xylostella was determined by the number of switches between herbivore guilds, the identity of the first and last herbivore as well as the specific order of herbivore attack.

First, the number of times a plant was exposed to the same feeding guild of attacker as part of sequential herbivore attack, 0-, 2- or 4-times phloem feeders or leaf chewers affected plant resistance to larvae of P. xylostella $\left(\mathrm{F}_{1,3}=3\right.$, $\mathrm{P}=0.04)$. Plants that were attacked four times by chewers tended to be more resistant to larvae of $P$. xylostella than plants exposed to four times attack by phloem feeders $\left(t_{1,2}=-1.67, P=0.097\right)$. Second, the feeding guild of the first attacker of the sequence did not affect plant resistance to larvae of P. xylostella (Figure 3a). However, the specific species identity of the first attacker of the sequence affected performance of $P$. xylostella (MLM: $\mathrm{F}_{1}, 4=2.89, \mathrm{P}=0.023$, Figure 3b). Plants that had been attacked first by $M$. persicae, were more resistant to larvae of P. xylostella independent of the order and identity of the second, 


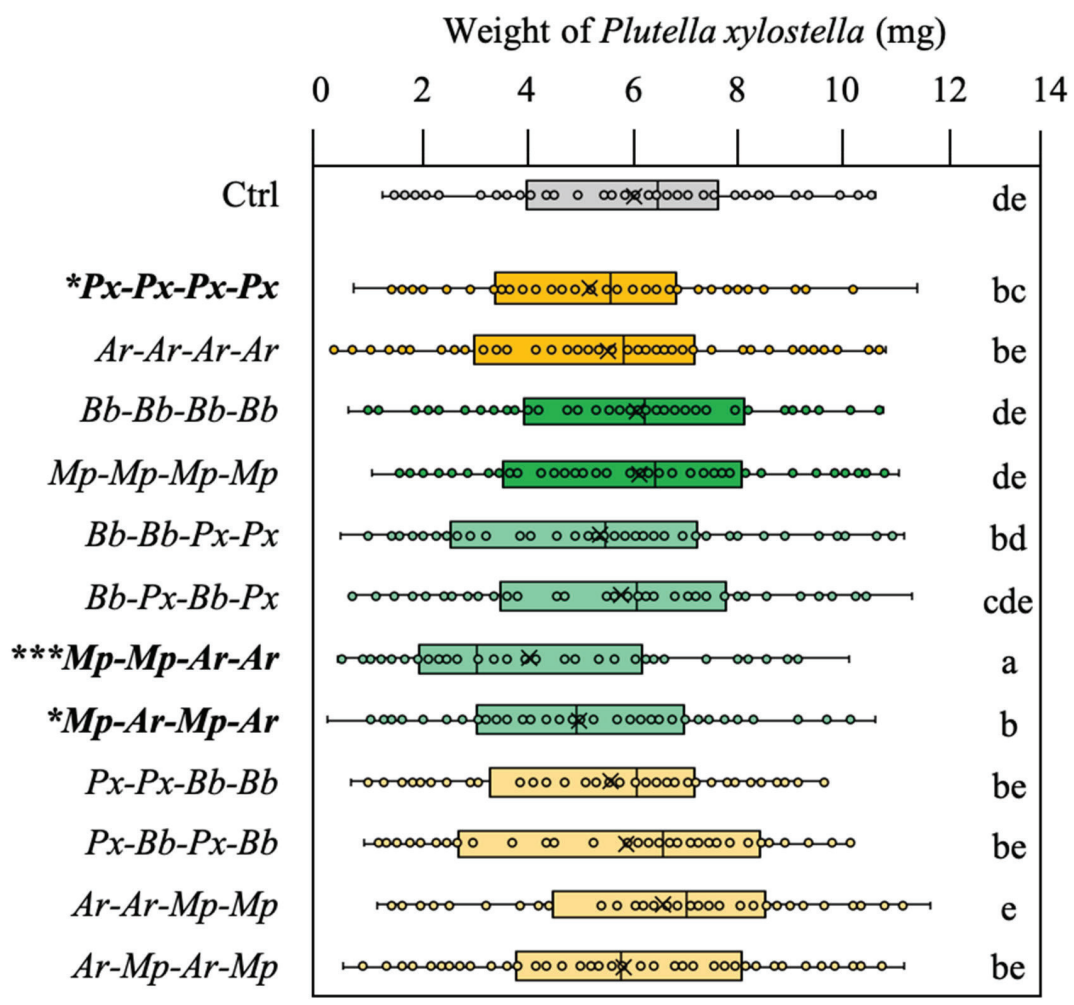

Figure 2. Weight (mg) of Plutella xylostella larvae after feeding for five days from Brassica nigra plants previously attacked by sequences of four herbivore events $(\mathrm{N}=18)$. Darker boxplots indicate herbivore sequences that received four times the same herbivore. Yellow or green bars indicate treatments where the first attacker was a chewer or a phloem feeder, respectively. Herbivore sequences that affected $P$. xylostella growth (compared to control, untreated plants) are marked in bold and with asterisks with significance levels $* P<0,05 ; * * * P<0,001$. Boxplot height corresponds to the first and third quartiles (Q1 and Q3), and the middle line to the median. Letters above the boxplots show significant differences (MLM, posthoc Tukey). Leaf chewers: Plutella xylostella $(P x)$ and Athalia rosae (Ar). Phloem-feeding aphids Brevicoryne brassicae (Bb) and Myzus persicae (Mp). 
third and fourth herbivore in the sequence $\left(\mathrm{t}_{1,2}=-2.33, \mathrm{P}=0.02\right.$, Figure $\left.3 \mathrm{~b}\right)$. Third, the feeding guild as well as the species identity of the last and thus fourth attacker of the sequence, affected plant resistance to P. xylostella larvae (MLM, Guild: $\mathrm{F}_{1,3}=-8.12, \mathrm{P}<0.001$; Identity: $\mathrm{F}_{1,4}=5.11, \mathrm{P}<0.0001$, Figure $\left.3 \mathrm{c}, \mathrm{d}\right)$. When the last attacker had been a chewer, plants were more resistant $P$. xylostella larvae (MLM, chewer $\mathrm{t}_{1,2}=-2.33, \mathrm{P}=0.021$ ) and these effects were particularly apparent for the leaf chewer $A$. rosae (MLM, $\mathrm{t}_{1,4}=-2.78, \mathrm{P}=0.006$, Figure $3 \mathrm{c}, \mathrm{d}$ ).

a)

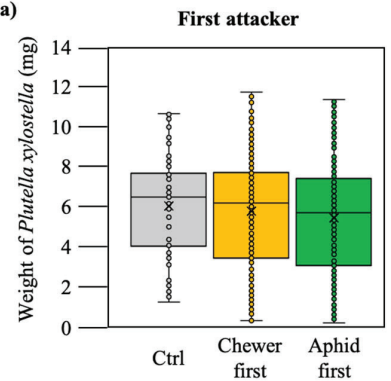

c)

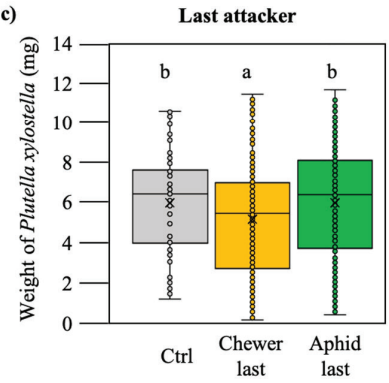

b)

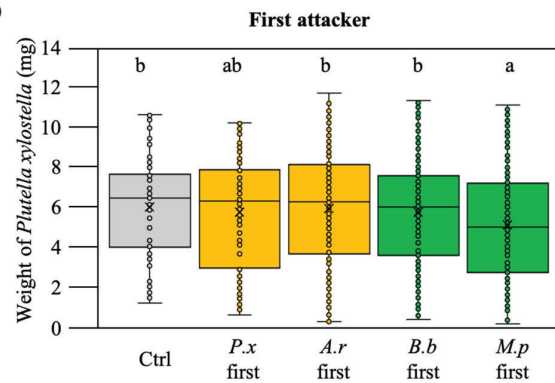

d)

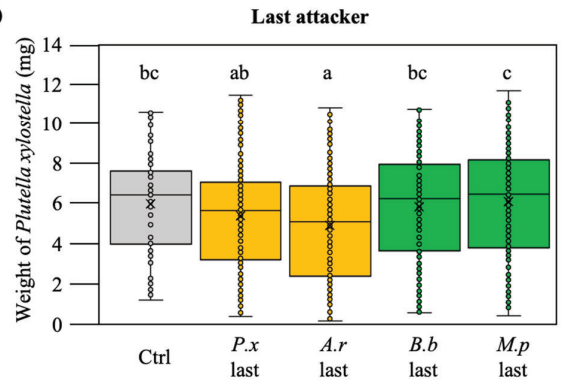

Figure 3. Effect of first or last attacker on resistance to Plutella xylostella. Weight (mg) of $P$. xylostella larvae after feeding as fifth herbivore from Brassica nigra plants that were previously attacked by four sets of herbivores where: a) the first attacker was either a chewer, an aphid or untreated plants (Ctrl). b) The first attacker was Plutella xylostella $(P x)$, Athalia rosae (Ar), Brevicoryne brassicae (Bb), Myzus persicae (Mp), or untreated plants (Ctrl). c) The last attacker was either a chewer, an aphid or untreated plants (Ctrl). d) The last attacker was Plutella xylostella (Px), Athalia rosae (Ar), Brevicoryne brassicae (Bb), Myzus persicae $(M p)$, or untreated plants (Ctrl). Boxplot height corresponds to the first and third quartiles (Q1 and Q3), and the middle line to the median. Letters above the boxplots show significant differences (MLM, posthoc Tukey). 
In summary, the history of sequential herbivore attack affected B. nigra resistance to larvae of $P$. xylostella. The number of times there was a chewer in the sequence, the species identity of the first attacker in the sequence and the guild and the identity of the last (i.e. fourth) attacker in the sequence affected the capability of plants to mount resistance to P. xylostella after previous exposure to multiple incidences of herbivore attack.

To further separate effects caused by number of switches between feeding guilds from effects by feeding guild, species identity and order of arrival, we analysed these effects within subsets of treatments that were equal in the number of herbivore switches. Brassica nigra plants attacked by sequences containing two times chewers, and two times phloem feeders, were more vulnerable to $P$. xylostella larvae when the first attacker was a chewer, compared with plants that were first attacked by a phloem feeder $\left(\mathrm{F}_{1,2}=10.85, \mathrm{P}=0.001\right)$. After first attack by a phloem feeder, the order of subsequent attackers influenced plant resistance to $P$. xylostella, as larvae grew more on plants attacked by the sequence aphid - chewer - aphid - chewer (B. brassicae - P. xylostella - B. brassicae - P. xylostella as well as $M$. persicae $-A$. rosae - M. persicae - A. rosae) than on plants attacked by the sequence aphid - aphid - chewer - chewer (B. brassicae - B. brassicae - P. xylostella - P. xylostella as well as $M$. persicae $-M$. persicae $-A$. rosae - A. rosae) (MLM, $\mathrm{F}_{1,2}=5.06, \mathrm{P}=0.03$; Figure $\left.4 \mathrm{a}\right)$. When the first attacker was the phloem feeder $B$. brassicae, plants were equally vulnerable to larvae of $P$. xylostella, irrespectively of the order of the subsequent attackers $\left(\mathrm{F}_{1,2}=1.34 \mathrm{P}=\right.$ 0.25 , Figure 4c). In contrast, when the first attacker was $M$. persicae the order of the attackers mattered and larvae of P. xylostella grew more on plants where the identity of the herbivore switched every time (larvae grew more on plants that had been exposed to $M$. persicae $-A$. rosae $-M$. persicae $-A$. rosae than on plants that had been exposed to $M$. persicae $-M$. persicae $-A$. rosae $-A$. rosae $)\left(\mathrm{F}_{1,2}=4.08\right.$ $\mathrm{P}=0.04$, Figure $4 \mathrm{~d}$ ). In contrast to the effect of herbivore order when the first attacker was an aphid, the order of herbivore arrival after the first herbivore was a leaf chewer did not affect plant resistance to $P$. xylostella larvae $\left(\mathrm{F}_{1,2}=0.75\right.$ $\mathrm{P}=0.39$, Figure 4b). These effects were also similar for the two leaf chewers $P$. 
a)

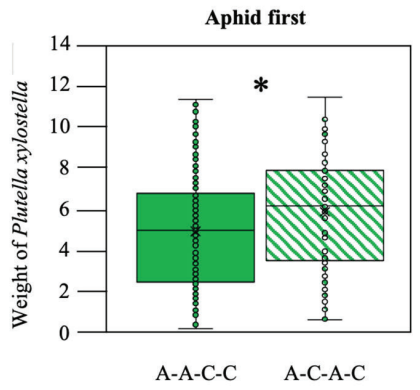

c)

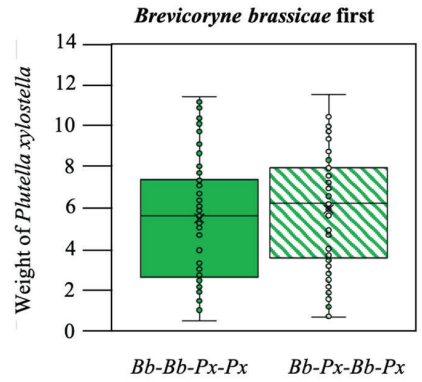

e)

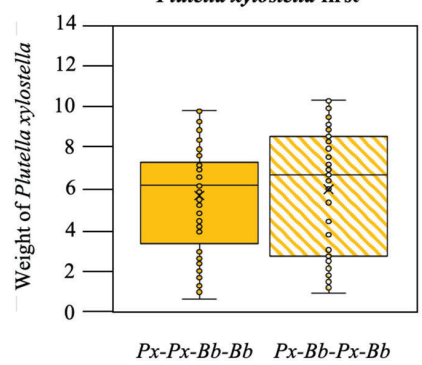

b)

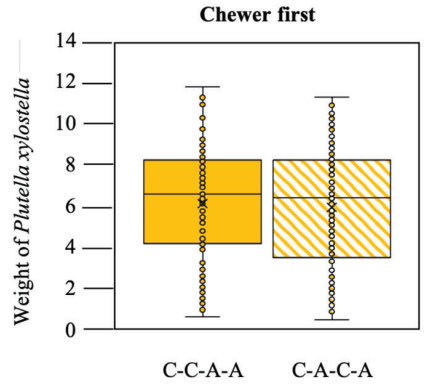

d)

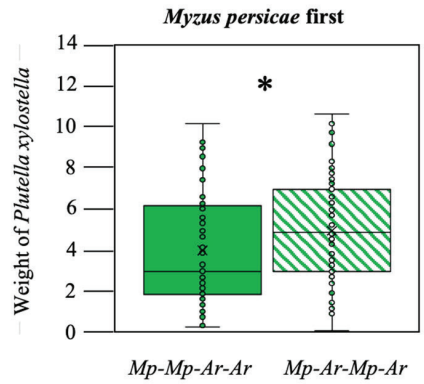

f)

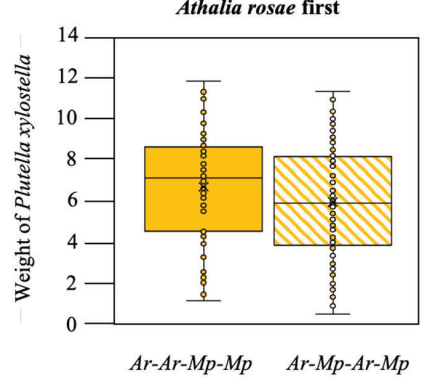

Figure 4. Weight (mg) of Plutella xylostella larvae after feeding for five days from Brassica nigra plants attacked by a sequence of herbivory with the guild or the species identity of first attacker constant but changing the order of the subsequent attackers of the sequence. First attacker: a) an aphid (A), b) a chewer (C), c) Brevicoryne brassicae $(B b)$ d) Myzus persicae (Mp), e) Plutella xylostella (Px), f) Athalia rosae (Ar). Boxplot height corresponds to the first and third quartiles (Q1 and Q3), and the middle line to the median. Asterisks show with significance differences * $P<0,05$. 
xylostella or $A$. rosae (P. xylostella first: $\mathrm{F}_{1,2}=0.05 \mathrm{P}=0.83 ; A$. rosae first: $\mathrm{F}_{1,2}=2.08$ $\mathrm{P}=0.15$, Figure $4 \mathrm{e}, \mathrm{f})$.

In summary, B. nigra plants attacked by sequences containing two chewers, and two phloem feeders, were more vulnerable to P. xylostella larvae when the first attacker was a chewer, compared with plants that were first attacked by a phloem feeder. Additionally, when plants were first attacked by an aphid, the order of subsequent attackers in the sequence influenced plant resistance. Plants that were attacked by the sequence aphid - chewer - aphid - chewer were more vulnerable to $P$. xylostella larvae than plants attacked by the sequence aphid aphid - chewer - chewer.

\section{DISCUSSION}

The objective of this study was to investigate plant resistance against a leaf chewing herbivore, after plants had been previously exposed to sequential attack by four other herbivores. We found that sequence of herbivore attack differentially affected $B$. nigra plant resistance to P. xylostella larvae. Four events of attack by $P$. xylostella induced resistance to larvae of P. xylostella compared to control plants. The number of times the plant was attacked by herbivores of the same feeding guild, the identity of the first attacker, the identity and the guild of the last attacker as well as the order of attackers within the sequence of multi herbivory influenced plant resistance to subsequent herbivory. The guild of the first attacker of the sequence did not affect plant resistance to P. xylostella. However, when plants had been first attacked by $M$. persicae they were more resistant to larvae of P. xylostella, regardless of the order of other herbivores attacking the plant. In contrast, the guild and identity of the last attacker influenced plant resistance to $P$. xylostella larvae as they grew less on plants where the last attacker of the sequence had been a chewer, especially when it was $A$. rosae. Our study shows that the sequence of herbivore attack is an important factor determining plant resistance to herbivores. 
In line with the induced defence hypothesis, B. nigra plants that had been attacked four times by caterpillars of $P$. xylostella were more resistant to caterpillars of P. xylostella feeding as fifth herbivore. However, when the aphid B. brassicae was introduced in the sequence of four attackers, the induced resistance to larvae of $P$. xylostella disappeared. The compromised induced resistance when there are other attackers than P. xylostella in the sequence of four attackers, suggests that B. nigra plants loose potential to deal with a specific herbivore attack when switching defence machinery towards other attackers in the sequence. Furthermore, four exposures to attack by the other leaf chewer (A. rosae) did not make the plant more resistant to P. xylostella caterpillars. The absence of induced resistance by the other chewer indicates that the induced response found on plants attacked four times by $P$. xylostella is not just a general defence mechanism in response to chewers, but that there is specificity in induced defence within feeding guilds. Similar specificity in induced resistance was found for Solanum dulcamara. Plants that had been damaged by the leafchewing beetle Psylliodes affinis were more resistant to P. affinis, while feeding by the leaf chewer Plagiometriona clavata did not induce resistance against $P$. affinis (Viswanathan et al., 2005). Our work identifies that specificity of induction by herbivore identity may be maintained under multi-herbivore attack.

Plants that were sequentially attacked four times by aphids defended equally well against larvae of $P$. xylostella compared with plants that had not been exposed to herbivory. This indicates that when the plant suffered four rounds of aphid attack, there was no aphid-induced susceptibility to a chewer. Several studies of plant responses to dual attack, have reported aphid-induced susceptibility to chewers, often supporting their findings based on the JA/SA negative crosstalk paradigm (Davidson-Lowe et al., 2019; Koornneef \& Pieterse, 2008; Li et al., 2014; Rodriguez-Saona et al., 2005; Soler et al., 2012). The absence of aphid-induced susceptibility to caterpillars or even presence of aphid-induced resistance to caterpillar attack may be caused by aphids depleting nutrients in the plant or by shifts in secondary metabolites (Jakobs et al., 2019). It is becoming clear that not only the guild of previous attackers is important in explaining 
plant resistance but many other factors such as the density of attackers influence the outcome of plant mediated interactions between herbivores (Kroes et al., 2015; Pineda et al., 2017). For resistance of B. nigra to sequential herbivore attack the prevalence of the second herbivore in the field is a more important driver of plant induced responses to the first attacker than the identity of the first herbivore itself (Mertens et al., 2021b).

Our work highlights that also the number of times the plant was attacked by an insect of the same feeding guild within the sequence of four attackers, influenced the plant's capability to respond to attack by P. xylostella. Caterpillars grew bigger when feeding on plants that had been exposed to four events of aphid infestation compared with those feeding on plants that had been exposed to four events of chewer attack or compared with plants that had been exposed to two rounds of herbivory by aphids plus two by chewers. This suggests that when the plant suffers attack by herbivores that arrive in sequences, being more times attacked by one type of insect, makes the plant more ready to defend against an insect of a similar type. Moreover, plants attacked by sequences containing two times chewers, and two times phloem feeders, were more vulnerable to $P$. xylostella larvae when the first attacker was a chewer, compared with plants that were first attacked by a phloem feeder. This suggests that the feeding guild of the first attacker may influence the plant's capability to defend against subsequent attackers. In line with our results, in maize the order of herbivore arrival was important in determining plant resistance to sequential attack. Spodoptera frugiperda attack induced resistance against larvae of Diabrotica virgifera virgifera, but only when S. frugiperda attacked the plant first (Erb et al., 2011). In our study, the species identity of the first attacker partly influenced plant resistance, as plants that had been attacked first by $M$. persicae were more resistant to P. xylostella larvae. Moreover, when the last attacker of the sequence was a chewer, especially when it was $A$. rosae, the plant was more resistant to P. xylostella larvae. Common garden experiments monitoring herbivore communities on B. nigra show that $M$. persicae is one of the first attackers colonising B. nigra plants, and that $A$. rosae and P. xylostella arrive later in the growing season of the plant (Mertens et al., 2021b). 
The fact that we found herbivore-induced resistance in response to exposure to herbivore sequences that are more commonly found in the field, suggests that B. nigra plants are adapted to the natural order of herbivore arrival (Mertens $e t$ al., 2021b).

Several studies on plant responses against single herbivore attack show canalization of plant responses, where plants attacked by a herbivore cannot fully defend after sequential attack (Soler et al., 2012; Viswanathan et al., 2007). Canalization may not be the optimal defence strategy in a scenario of multiple attack by herbivores that arrive sequentially. If the plant completely directs its defence machinery towards the first herbivore, and cannot switch response to the upcoming attackers, the plant may be undefended against later arriving herbivores. Our work does not show evidence for canalization of plant defences in B. nigra, as there is no induced susceptibility to $P$. xylostella larvae by sequences of four herbivores, compared with undamaged plants. Consequently, when facing herbivory by multiple insects that arrive in sequences, the ability of $B$. nigra plants to defend against a late arriving herbivore, is not hampered. Additionally, our data suggest that $B$. nigra plants do not fully switch their resistance phenotype to a new attacker, i.e. plants that had been attacked four times by $P$. xylostella were more resistant to larvae of $P$. xylostella but when there was a switch of attackers in between, the induced resistance was reduced. Furthermore, $P$. xylostella larvae grew better on plants attacked by the sequence aphid - chewer - aphid - chewer than on plants attacked by the sequence aphid - aphid - chewer - chewer. The reduced resistance of plants that had been exposed to more switches of attackers, further supports that B. nigra plants are limited in showing a full defence response when the attacker changes several times.

To conclude, we show that history of sequential attack is an important factor determining plant resistance to its community of attackers. The relative importance of overlapping herbivore populations, densities, timing of their arrival and plant ontogeny, in addition to patterns in incidence we tested here, should be evaluated in future studies. In depth studies on the physiological 
changes after exposure to each newly arriving herbivore should shed light on how plants regulate plasticity to multi-herbivore attack. Additionally, it is crucial that further studies explore plant adaptation to multi-herbivore attack under field conditions. This could be done by studying the herbivore communities forming on plants previously induced by different sequences of herbivory (Stam et al., 2018) assessing the importance of the first attacker, the last attacker and the order of attackers within the sequence and assessing the consequences for other community members and on plant fitness.

ACKNOWLEDGEMENTS The authors want to thank the Unifarm team for growing the plants, especially Sean Geurts and André Maassen. We thank Marcel Dicke for constructive comments on an earlier version of the manuscript. 

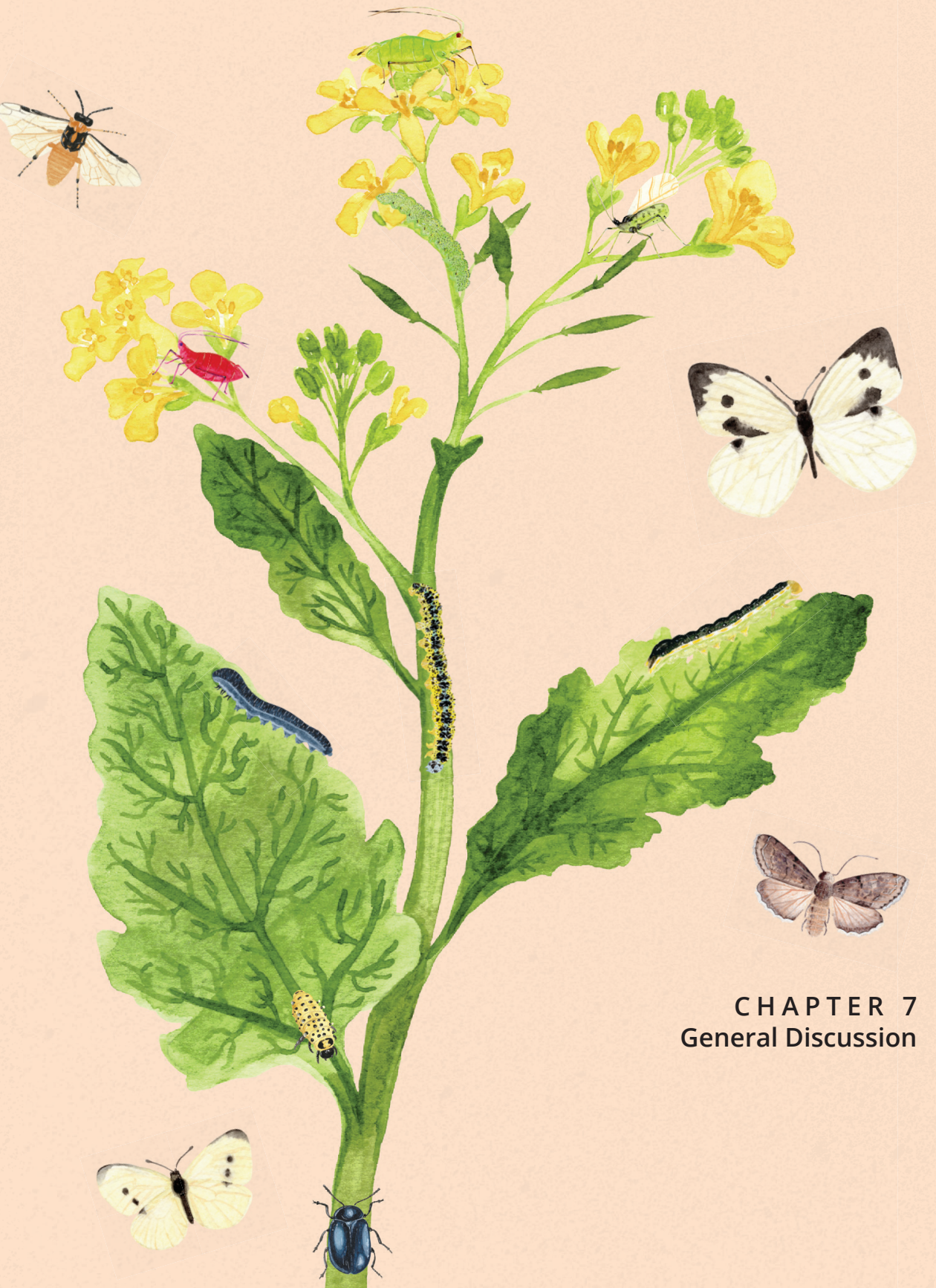



\section{INTRODUCTION}

In terrestrial ecosystems, plants are at the basis of many food chains. Plants are the food source, mating place, or a shelter to hide from predators for many organisms. Consequently, plants are central in food webs and mediate many direct and indirect interactions between organisms. Insects are one of the most important groups of organisms interacting with plants. It is estimated that there are up to five million species of insects on Earth, of which $50 \%$ are herbivores (Schoonhoven, van Loon, Dicke, 2005). Therefore, interactions with insect herbivores are very common, and represent a strong selection force on plant traits. Insects represent one of the most diverse groups of organisms, and this diversity is reflected in the array of strategies they have evolved to exploit plants. Given that in nature plants are under the attack by multiple herbivores, plants also evolved a repertoire of defence strategies to prevent their tissues from being consumed. Defending against multi-herbivore attack is not an easy task, as it implies being able to counteract a diversity of stressors that range from a microscopic bacterium to a big mammal.

In my $\mathrm{PhD}$ project, I explored how Brassica nigra plants deal with individual or simultaneous attack by its most common herbivores. In this chapter I will discuss: 1) The molecular regulation of how plants deal with multi-herbivore attack; 2) Challenges for plants of dealing with multi-herbivore attack; 3) Specificity of plant phenotypic changes induced by herbivory; 4) Plant strategies to optimise defence responses to multiple attackers; 5) Ecological consequences of induced plant responses to multiple attack; I end this general discussion with a perspective for future research.

\section{MOLECULAR REGULATION OF HOW PLANTS DEAL WITH HERBIVORE ATTACK}

Plants interact with a diverse community of attackers that may all require different defensive traits. Therefore, plants are under selection to recognize the specific attacker by its feeding guild (e.g. leaf chewer or phloem feeder), feeding pattern, feeding position, or elicitors in its saliva and mount a defence 
response. Induced defence theory posits that plants tailor the induced-defence phenotype to the specific attacking herbivore (Erb et al., 2012; Kliebenstein, 2014). However, how specific the plant phenotype is to the attacker remains to be investigated. My work has identified that some of the molecular responses of B. nigra to herbivore attack are attacker specific, although there are guildspecific responses as well (chapter 3 and chapter 4). In chapter 3 I show that, in line with many studies that show that chewers induce JA (Erb et al., 2012), B. nigra plants induce the expression of a JA-marker gene upon attack by the chewers Athalia rosae, Pieris brassicae and Plutella xylostella. In contrast, the expression of a JA-marker gene was not affected by the chewers Autographa gamma, Mamestra brassicae and Pieris rapae. This lack of JA induction by the latter chewers could be attributed to a limited leaf consumption by those insects, or to a site-dependent induction, as has been reported in other studies (Bonnet et al., 2017; Chen et al., 2020; Chrétien et al., 2018). In chapter 3 I show that, in line with literature (Appel et al., 2014), chewers induce a JA-biosynthetic gene, and phloem feeders induce a SA responsive gene. However, this was only true when the chewers where specialists as generalist chewers did not induce JA compared to phloem feeders. The expression of an SA-responsive gene was lower in plants attacked by generalist leaf chewers than in plants induced by specialist and generalist phloem feeders, whereas it did not differ between specialist chewers and phloem feeders. Thus, I show that the molecular responses to single attack partly depend on the degree of specialisation of the attacker.

In chapter 4, I went one step further, and challenged plants with one, two or four species of chewing insects, phloem-feeding insects or a combination of both. Compared to undamaged plants or to plants that were attacked by phloem feeders, all the chewers tested induced the JA-biosynthetic gene (LOX2) as well as the JA-responsive gene (VSP2). In contrast with chapter 3, where I found that feeding by the chewer $M$. brassicae did not induce JA, in chapter 4, M. brassicae induced the expression of two JA-related genes. This inconsistency between the two chapters could be explained by the differences in the set-up for the molecular analysis. While in chapter 3 the insects were allowed to freely feed 
from the plant and the youngest fully-expanded leaf was sampled for genetic analysis, in chapter 4 the insects were constrained to one leaf, and that same leaf was sampled. As M. brassicae has a very spread feeding pattern (personal observation), the leaves sampled for chapter 3 likely had lower local induction than the ones sampled for chapter 4 . This indicates how the methodology can influence molecular results and the importance of taking this into consideration when designing experiments. Even though in chapter 4 I show that all chewers tested induced JA-regulated responses, I also show specificity in the response to species or their combination. I found that simultaneous attack by two chewers induced LOX2 and VSP2 more strongly than single attack by one chewer, than simultaneous attack by four chewers or than attack by a mix of chewers and phloem feeders (Fernández de Bobadilla et al., 2021). Similarly, Brassica juncea plants simultaneously attacked by two chewers had a stronger defence induction (alkenyl glucosinolate production and trichome density), compared with plants under single attack (Mathur et al., 2013).

The current paradigm is that plant responses to phloem-feeder attack are regulated via the SA-pathway (Erb et al., 2012; Jaouannet et al., 2014; Züst \& Agrawal, 2016). Moreover, it has been proposed that the phloem-feeder suppression of JA could be due to negative crosstalk between the JA and SA pathways (Soler et al., 2012; Züst \& Agrawal, 2016). However, experimental evidence for SA/JA antagonism remains inconsistent (Broekgaarden et al., 2011; Y. Li et al., 2016). In line with the JA/SA negative crosstalk paradigm (Johnson et al., 2020; Schweiger et al., 2014; Zarate et al., 2007) I show in chapter 4 that feeding by one or by four species of phloem feeders suppressed the expression of a JAresponsive gene compared with undamaged plants, or with plants treated with chewers or with a mix of chewers and phloem feeders. Nonetheless, in constrast with the studies that claim that phloem feeders induce SA, in chapter 3 and chapter $4 \mathrm{I}$ show that in B. nigra, the expression of the SA-responsive gene (PR1) was not induced by any of the phloem feeders, compared to expression levels of these genes in undamaged plants. Surprisingly, the only study that investigated $B$. nigra transcriptomic responses to phloem-feeder attack, shows that the responses 
involve the JA and the ET pathway (Broekgaarden et al., 2011). The expression of the SA marker gene (PR1) was induced by chewers and by a mix of chewers and phloem feeders (chapter 4). In contrast with my results, another study on $B$. nigra found that feeding by the chewer $P$. rapae did not affect expression of the SAmarker gene PR1 (Broekgaarden et al., 2011). However, other studies reported SA and JA induction upon leaf-chewer feeding (Arena et al., 2018; Heidel \& Baldwin, 2004; Kiełkiewicz et al., 2019; Y. Li et al., 2016). This disparity in results might be attributed to different induction times. Interestingly, in chapter $4 \mathrm{I}$ show that the phloem-feeder suppression of JA-responses also occurs when phloem feeders and chewers simultaneously attack the plant. The expression of two JA-marker genes was lower on plants under simultaneous attack by phloem feeders and chewers, compared to plants only attacked by chewers, which might be interpreted as JA/ SA negative crosstalk. The molecular regulation of plants simultaneously attacked by insects of different feeding guilds has rarely been investigated. The few studies available show that when phloem feeders are introduced to the plant together with chewers, many genes are suppressed compared to single-guild attack (RodriguezSaona et al., 2010).

Thus, plant molecular regulation of responses to herbivore attack is not as simple as chewer induced-JA/phloem feeder induced-SA. Some studies have investigated plant transcriptomic responses of plants under dual attack (Bonnet et al., 2017; Coolen et al., 2016; Davila Olivas et al., 2016; Kroes et al., 2017). However, the molecular regulation of plants attacked by more than two herbivores (i.e. multi-herbivore attack) has never been explored. Further research should investigate the molecular regulation of plant responses to multiple herbivores of the same or of different feeding guilds that arrive sequentially, as well as the role of herbivore instar, plant ontogeny, combination of stresses (both biotic and abiotic). In this respect, transcriptome analysis of plants under multi-herbivore attack will give a more complete view of the molecular regulation of plants against multiple attackers and give some hints on important regulators in defence against phloem feeders, chewers or a mix of both. Additionally, the molecular regulation of simultaneous attack by insects 
of different feeding guilds may be different across plant ontogeny, plant species or depend on attacker identity.

\section{CHALLENGES IN DEALING WITH MULTI-HERBIVORE ATTACK}

Plant resistance to herbivory is modulated by the identity (Agrawal et al., 2014; Van Zandt \& Agrawal, 2004), guild (Bidart-Bouzat \& Kliebenstein, 2011; Davidson-Lowe et al., 2019), and the density of the attacker (Kroes et al., 2016; Kroes et al., 2015; Pineda et al., 2017; Ramirez \& Eubanks, 2016), among others factors. Only recently, studies including more than two attackers have unravelled that the order of attackers also influences a plant's ability to defend against attackers (Stam et al., 2017, 2018, 2019; Viswanathan et al., 2007). However, many aspects of multi-herbivore attack remain to be investigated. Among them, the role of herbivore richness on plant defence had never been investigated. In chapter 4, I show that the richness of attackers as well as their feeding guild determine a plant's ability to defend against herbivores. Increased richness in phloem feeders compromises plant resistance to a chewer. Plants simultaneously attacked by four species of phloem feeders become more susceptible to a chewer, whereas this is not the case for plants attacked by one or by two species of phloem feeders. The lack of induced susceptibility to a chewer by one or by two species of phloem feeders in B. nigra contrasts with previous studies on other plant species (Soler et al., 2012) and shows that responses to herbivores are often plant species-specific (Hervé \& Erb, 2019; B. Li et al., 2018). In line with previous studies reporting chewer-induced resistance to a chewer, in chapter 4 I show that leaf chewers induce resistance to subsequent attack by a chewer. Interestingly, chewer richness does not play a significant role in chewing herbivore induced responses. Attack by a mix of herbivores from different feeding guilds results in plant resistance similar to resistance levels of plants that were not previously exposed to herbivory. Very few studies investigated resistance of plants attacked by insects of two different feeding guilds and, in line with my results, these studies generally show an attenuation on plant defence traits on mixed induced plants, compared to 
chewer induced plants (Ali \& Agrawal, 2014; Delphia et al., 2007; RodriguezSaona et al., 2003, 2005; Rodriguez-Saona et al., 2010). The absence of induced susceptibility or induced resistance in plants attacked by a mix of chewers and phloem feeders was true for most of the combinations of chewers and phloem feeders.

In addition to herbivore richness, I have unravelled that history of sequential herbivore attack differentially affects a plant's capability to defend itself against a subsequent herbivore (chapter 6). The number of times the plant was attacked by herbivores of the same feeding guild, the identity of the first attacker, the identity and the guild of the last attacker as well as the order of attackers within the sequence of multiple herbivores influences plant resistance to subsequent herbivory.

In summary, in this thesis I have shown that the natural prevalence of herbivores, feeding guild, their identity, richness and the order of the attackers affect plant ability to defend against a late arriving herbivore. My thesis shows that to understand the evolution of induced defence strategies plant responses to multi-herbivore attack should be evaluated in a community context including sequential herbivores of different feeding guilds. The role of herbivore instar, plant ontogeny, plant species and combination of stressors (both biotic and abiotic) remains to be investigated.

\section{SPECIFICITY OF PLANT PHENOTYPIC CHANGES INDUCED BY HERBIVORY}

The molecular mechanisms to defend against attackers regulate the expression of plant phenotypic traits. Among the most important traits to defend against herbivores are physical barriers, secondary metabolites and defence proteins. Importantly, defence traits can be induced or repressed upon herbivory (Karban, 2011). Many studies claim that plants tailor their response to the specific attacker. There is extensive evidence for specificity in plant recognition of the attacker (Santamaria et al., 2021), however, the proof 
for specificity in plant response in terms of plant phenotype is lacking (Erb et al., 2012). By studying the performance of a second attacker on plants induced with different herbivore treatments, in this thesis I studied some components of specificity of plant-induced responses to herbivory. In chapter 3, I show that even though the molecular plant responses to herbivores are specific to the feeding guild of the attacker, the induced resistance to a second attacker is not specific to the feeding guild nor to the identity of the first attacker. Strikingly, the induced resistance to one herbivore is better explained by the prevalence of the second herbivore in the field (Mertens et al., 2021). By showing that feeding guild of the initial attacker is not explaining resistance to a second attacker I challenge the current paradigm on plant limitations to deal with sequential attack by insects of different feeding guilds (Moreira et al., 2018; Thaler et al., 2012). A general defence phenotype that makes the plant resistant to the more common attackers is likely more adaptive than a tailormade response to every single attacker. In chapter 4 , I challenged plants with 51 herbivore treatments of one, two, or four herbivore species of chewers, phloem feeders or a mix of both and studied resistance to caterpillars of $P$. xylostella, one of the most prevalent herbivores interacting with $B$. nigra plants (Mertens et al., 2021). I found that there is some degree of specificity, even though all the chewer treatments induce a general JA-regulated response and make the plant more resistant to a second attacker, compared with untreated plants (Fernández de Bobadilla et al., 2021). Plants simultaneously attacked by A. rosae and Phaedon cochleariae were more resistant to a second attacker than plants simultaneously attacked by the four chewers (A. rosae - M. brassicaeP. brassicae- P. cocbleariae). Likewise, in a different study Brassica juncea plants simultaneously attacked by two chewers had a strong defence induction (alkenyl glucosinolate production and trichome density), compared with plants under single attack (Mathur et al., 2013).

Many studies indicate that, although attacker recognition, and the molecular regulation of the response are specific, the final plant phenotype in response to very different organisms is similar. One striking example is the 
response of Arabidopsis plants to insect eggs, being very similar to PAMPtriggered response to pathogens (Gouhier-Darimont et al., 2013; Hilker \& Fatouros, 2015). Probably, plants do not have a regulatory network to respond to every unique organism, but rather an integrated network that orchestrates the response to multiple attackers. Likely plants are equipped with a suite of defence traits, and under herbivory induce the most effective combination of traits to minimize the fitness loss. An important question to ask is why there is specificity in recognition if there is not specificity in response. For example, several studies show that plants can differentiate between mechanical damage, damage by chewing insects, and between insect-associated cues (frass, vibrations) (Acevedo et al., 2015; Appel \& Cocroft, 2014; Body et al., 2019; Ray et al., 2015) but the changes in phenotype to these cues are not so specific. One explanation would be that a general recognition system to differentiate between self and non-self is not enough to tell apart beneficial from detrimental organisms. However, a general response is probably effective against most of the attackers. Plants interact with phylogenetically very similar microorganisms that can range from beneficial to pathogenic. To avoid letting a pathogen in, mounting a hypersensitive response against a beneficial microorganism, plants need a very specific recognition system. Actually, when looking at plant interactions with beneficial microorganisms, initial recognition of MAMPs by the plant results in a mild induction of immune responses (Van Wees et al., 2008) and to have a successful colonisation, the microorganisms need to manipulate the plant immune system (Zamioudis \& Pieterse, 2012). Thus, plant responses to different attackers/organisms can be very specific and also be changed by the organism themselves, generating an extensive array of multiple scenarios for plant responses.

\section{PLANT STRATEGIES TO OPTIMISE DEFENCE RESPONSE TO MULTIPLE ATTACKERS}

The big diversity of herbivores and ways they exploit plants challenges plants to express an optimal defence phenotype. On the one hand, plants may be genetically limited, and lack the specific genetic pathway to produce 
the appropriate response against a specific insect. In other cases, plants may have the defence pathway to target one insect, but the limitation comes in terms of antagonistic crosstalk between phytohormones. This negative communication may impair plant responses to one insect when the plant has already responded to a different insect. Importantly, insects are sensitive to different plant traits and often ecological trade-offs occur, where by gaining resistance to one herbivore plants are more vulnerable to others, or become less attractive to beneficial insects such as natural enemies of the herbivores and pollinators. To mention some of the challenges plants face, herbivore attack may vary in terms of the type of attacker (i.e. feeding guild or identity), the timing and order of arrival, the abundance, species richness, and plant parts attacked. The high diversity of stressors surrounding plants, makes it imperative that plants have evolved several strategies to survive. Plants need to use their resources efficiently, and accurately, and the strategy played will depend on the combination and type of stressors they are facing. In chapter 2, I propose several strategies that plants may have, to deal with a dynamic community of multiple attackers (Fig. 1). These defence strategies could be seen as risk management tools to deal with a multitude of stressors. In this thesis I identify some of the strategies listed in B. nigra plants. Additionally, I have found evidence from other studies that show the strategies in other plant species.

I show that $B$. nigra plants canalise their defence response to the first attacker as chewers induce resistance to a chewer and phloem feeders induce vulnerability to a chewer (chapter 4). The canalisation strategy has been reported in several plant species belonging to diverse families such as the Brassicaceae, Apocynaceae, or Solanaceae (Ali \& Agrawal, 2014; DavidsonLowe et al., 2019; Soler et al., 2012). However, under multiple attack by attackers arriving in sequences, $B$. nigra plants did not canalise their phenotype to the first attacker, as there is no induced susceptibility to a herbivore after sequences of four herbivores, compared with undamaged plants (chapter 6). In chapter 4, plants were attacked by the herbivores simultaneously, whereas in chapter 
6 the herbivores were introduced in sequences of attack by four herbivores. During the growing season of $B$. nigra, plants receive sequential attack by many herbivores, and therefore the herbivore treatments applied in chapter 6 resemble more closely the natural situation. The induced susceptibility after being simultaneously attacked by four species of phloem feeders could be an artefact, as this situation barely happens for B. nigra in natural ecosystems. A more common situation in nature would be B. nigra plants being attacked by a mix of chewers and phloem feeders, and in this situation, there was no herbivore-induced susceptibility (chapter 4).

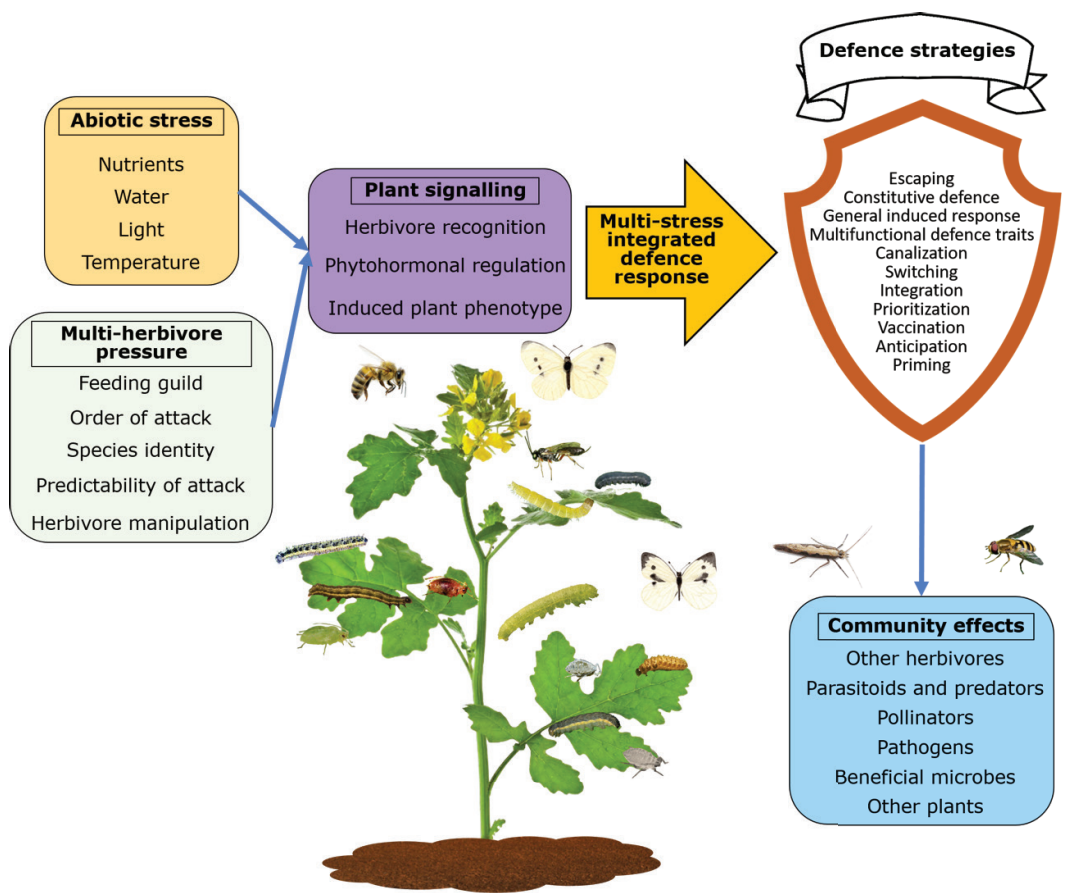

Figure 4. Plants experience multiple biotic and abiotic stresses during their lifespan. All these stresses are expected to be integrated and will determine which defence strategy is played by the plant. 
Brassica nigra plants integrate responses to two different attackers when simultaneously attacked by chewers and phloem feeders, as plants showed an intermediate phenotype compared to plants attacked by either chewers or phloem feeders (chapter 4). Likewise, other plant species integrate responses to double attack by chewers and phloem feeders, and attenuate JA-related responses to chewers in the presence of phloem feeders (Ali \& Agrawal, 2014; Kiełkiewicz et al., 2019). Additionally, chapter 6 suggests that B. nigra plants do not fully switch their resistance phenotype to a new attacker. Plants that had been attacked four times by $P$. xylostella were more resistant to larvae of $P$. xylostella but when there was a switch of attackers in between, the induced resistance was reduced. Furthermore, P. xylostella larvae grew better on plants attacked by the sequence phloem feeder- chewer - phloem feeder- chewer than on plants attacked by the sequence phloem feeder- phloem feeder- chewer - chewer. The reduced resistance of plants that had been exposed to more switches of attackers, further supports that B. nigra plants are limited in showing a full defence response when the attacker changes several times. In contrast, Arabidopsis thaliana plants under sequential stress are able to switch their defence machinery towards the second stress (Coolen et al., 2016). Transcriptomic analysis of $A$. thaliana during sequential biotic and abiotic stresses showed that even though individual stresses triggered very different transcriptomic profiles, when two stresses were applied in sequence, plants displayed transcriptome profiles that were very similar to the second stress, irrespective of the nature of the first stress (Coolen et al., 2016). Evidence shows that plants can remobilize and reallocate resources from secondary to primary metabolism and vice versa (Erb \& Kliebenstein, 2020). Probably this is also the case in the context of switching the response to different attackers where plants could produce chemicals in response to the current attacker, and when a different attacker comes, and it needs another response, reabsorb the first compound and use it to produce new defence compounds (Bekaert et al., 2012). Biosynthetic enzymes and intermediates are shared between pathways (Tholl et al., 2005), reducing the costs of switching responses between attackers. Also, multi-functional enzymes with broad substrate specificity may reduce costs of switching because fewer intermediates are required (Tholl et al., 2005). 
Among the plant-associated herbivore community members, some attackers are more damaging than others (Poelman \& Kessler, 2016) and plants may use the prioritization strategy, and wait for their attack to induce a defence response. This strategy can also be used to prioritize defence of tissues which loss imply a high fitness cost (e.g. flowers) (Chrétien et al., 2018; Keith \& MitchellOlds, 2017). Strikingly, I show that B. nigra plants anticipate and prepare for the attack of the more damaging herbivores (chapter 3 ) as plants are more resistant to sequential attack by herbivores that are more common in the field (Mertens et al., 2021). Plants responding to insect cues (damage or egg deposition) and inducing resistance against future attack are using the anticipation strategy as well (Dalin \& Björkman, 2003; Orrock et al., 2018; Pashalidou et al., 2015). Other studies have shown plants to be equipped with the vaccination strategy inducing a response to a herbivore species that can be tolerated with the benefit of reduced attack by a more ravaging herbivore (Kessler \& Baldwin, 2004). Some authors even claim that some environmental cues can prime plants and prepare them for future attack (Conrath et al., 2006; Martínez-Medina et al., 2016).

The array of plant strategies against insect herbivores that I identified in chapter 2, are likely involved in defence against other herbivores as well. As in nature plants encounter simultaneously or sequentially very diverse biotic and abiotic stressors, the optimal phenotype will likely include integration of resistance to all of these stressors. Presumably, plants are equipped with a defence network machinery that integrates responses to individual stresses. In the past years a few studies have started exploring plant transcriptomic responses to stressors of different nature. Undoubtedly, the plant regulation of defence against a combination of stressors is very different from that against a single stress (Coolen et al., 2016; Davila Olivas et al., 2016; Davila Olivas, Kruijer, et al., 2017). However, we are far from understanding the regulatory network underlying responses to a combination of attackers. More importantly, there is no evidence on whether the induced plant phenotypic changes are -as many studies claim- "tailor-made" to the stressor, or rather a general phenotype that targets all of them (Jones \& Dangl, 2006; Voelckel \& Baldwin, 2004; Vogel et al., 2007). 
Presumably, the expressed plant phenotype does not target individual stressors. It has already been shown that plant traits against slugs often make plants more resistant against insects (Calf et al., 2018; Hanley \& Sykes, 2009; Hanley et al., 2018; Loy et al., 2017; Roda et al., 2019). There is also extensive evidence showing that pathogens and insects interact via plant-mediated changes (Badri et al., 2013; Desurmont et al., 2016; Eberl et al., 2018, 2020; Franco et al., 2017; Guo et al., 2014; Hauser et al., 2013). Therefore, a defence strategy making plants resistant against both insects and pathogens is likely adaptive. It has even been shown that plants use identical inhibitors to restrict insect feeding and pathogen infection (Kirsch et al., 2020). In order to truly understand plant-trait selection, future studies should embrace the complexity of plant interactions with all stressors and friends. Exciting discoveries on plant strategies to their biotic and abiotic environment are expected to result from this.

Given the evidence of plants having several strategies to counteract herbivory, the pertinent question to ask is whether individual plants are equipped with a full set of strategies or only have a few. Additionally, we should question if plants that have evolved under different herbivore communities have similar strategies (Davila Olivas et al., 2017). If plants lack any of the strategies the question is why selection has eliminated some, and on which basis. Possibly, plants exhibit different strategies depending on the situation (i.e. herbivore pressure and abiotic stress), and can even exhibit different strategies simultaneously in different plant parts. For example, plants may exhibit local canalisation (Chen et al., 2020) in one leaf attacked by a very damaging herbivore and integration in another leaf attacked by two different herbivores.

\section{ECOLOGICAL CONSEQUENCES OF PLANT INDUCED RESPONSES TO MULTIPLE ATTACK}

In natural ecosystems, plants are central in a network of species that interact directly or indirectly via the plant (Ohgushi, 2005). Herbivores induce dramatic changes in a plant phenotype and alter the way plants are perceived by other organisms thereby altering community dynamics. Importantly, 
herbivore-induced changes in plant phenotype strongly affect the foraging of later arriving herbivores, parasitoids and pollinators (Chrétien et al., 2021; Rusman et al., 2018; Soper Gorden \& Adler, 2018). In chapter 5, I show that plants treated with herbivores were more attractive to a later arriving herbivore in the field. Likewise, in a field study P. xylostella was more abundant on Brassica oleracea plants attacked by $P$. rapae, compared with undamaged plants (Poelman et al., 2008). Strikingly, in my thesis I show that the positive effect of herbivory on colonisation by a later herbivore is maintained regardless of the guild and the richness of species of the initial herbivores (chapter 5). The consistently higher abundance of P. xylostella on herbivore-treated plants could be attributed to a herbivore-induced change in important plant traits for insect foraging. One possible explanation for $P$. xylostella preferring herbivoreinduced plants is that the herbivore-damaged plants might be more detectable because of a higher HIPV emission (El-Sayed et al., 2016). Alternatively, plants that received herbivory might represent an enemy-free space for $P$. xylostella (Dicke et al., 2009; Stamp, 2001). In my thesis I have not recorded differences in P. xylostella colonisation between the investigated herbivore combinations in the field (chapter 5). This lack of specificity, partly contrasts with my findings of chapter 4, where P. xylostella performs better on plants attacked by the highest species richness of phloem feeders, whereas the performance was reduced on plants attacked by chewers, irrespectively of the richness of attackers (Fernández de Bobadilla et al., 2021). Further research should disentangle which plant traits mediate the differences in P. xylostella performance found in chapter 4 , that do not correspond with the difference in abundance found in chapter 5 .

Importantly, herbivore-induced changes in plant traits can also affect higher trophic levels. However, in contrast to the consistently stronger abundance of P. xylostella in herbivore-treated plants, compared to untreated plants the parasitism of P. xylostella was not higher in all the herbivore treated plants, and it was higher in some of the herbivore-treated plants than in others (chapter 5). Thus, I found specificity in the herbivore-induced changes in 
important cues for parasitoid foraging. Other studies found that parasitoids are able to discriminate between plants that have been attacked or not, between plants under single or dual attack, and even on the type and density of attackers (Blubaugh et al., 2018; Bukovinszky et al., 2012; De Boer et al., 2008; de Rijk et al., 2016; De Rijk et al., 2013; Ponzio et al., 2016; Rodriguez-Saona et al., 2003; Rodriguez-Saona \& Thaler, 2005). Nonetheless, the importance of species richness and traits for parasitoid attraction under field conditions was never explored. Interestingly, the specificity in parasitism rate that I report in chapter 5, was not explained by the guild of the attackers. Compared with untreated plants, I found a higher degree of parasitism not only on plants attacked by some of the chewers, but also on plants attacked by some of the phloem feeders. The highest parasitism rate was found on plants induced by a mix of two chewers and two phloem feeders. One potential explanation for the differential attraction of parasitoids to mixed-induced plants compared to single-guild induced plants is the effect that the mixed induction may have on the volatile blend of the plant. For example, cotton plants under dual attack by caterpillars of Spodoptera exigua and by the phloem-feeding insect Bemisia tabaci emitted lower amounts of volatiles compared with plants only attacked by caterpillars (Rodriguez-Saona et al., 2003). Presumably depending on the herbivore species in the mix, and on the parasitoids tested as responder, the attraction is enhanced or reduced (Dicke et al., 2009). The higher attraction towards mixed-induced plants from our study could be due to a higher detectability of the volatiles from mixed-induced plants (Aartsma et al., 2019). Additionally, the parasitoids may associate the mixed-induced volatiles with a higher quality of the host. In chapter 4 , I showed that compared to the performance on undamaged plants, P. xylostella grew less on plants induced by chewers, while on mixed-induced plants the performance was not reduced (Fernández de Bobadilla et al., 2021).

On top of the herbivore-induced effects on later arriving herbivores and parasitoids, pollinator visitation to $B$. nigra plants changes depending on the feeding guild, and the species richness of the herbivores attacking the plant 
(chapter 5). Previous research on effects of single herbivore-induced changes on pollinator visitation show that the pollinator community of $B$. nigra differed depending on whether plants were uninfested or infested with a chewing herbivore, but it did not change when they were attacked by phloem feeders (Rusman et al., 2018). In contrast, I found that the pollinator community is also affected by phloem-feeder infestation, compared to the community visiting flowers on untreated plants (chapter 5). Interestingly, not only the richness of herbivores affected the pollinator visitation, but also species identity. While in general herbivory affects pollinators, some of the changes depend on the herbivore species. This herbivore-specificity contrasts with other studies that could not detect differences in the pollinator visitation between plant treatments with individual herbivore species (Rusman et al., 2018). The changes in pollinator visitation found in our study may be mediated by herbivore-induced changes in floral traits, e.g., flower phenology, flower number and morphology, presence of deterrents in pollen and changes in flower volatiles (Kessler \& Chautá, 2020; Moreira et al., 2019).

In my thesis I have shown that herbivory affects plant interactions with other herbivores, with higher trophic levels and with pollinators (chapter 5). Importantly, I show that this change in interactions has consequences for plant fitness. Other studies reported herbivore-induced effects on plant fitness in the field (Cozzolino et al., 2015; Devegili \& Chalcoff, 2020; Lankau \& Strauss, 2008; Soper Gorden \& Adler, 2018). However, very rarely has it been studied how plants maximise fitness when defending against attack by diverse assemblages of herbivores while maintaining the attraction of parasitoids/predators and pollinators. I show that the composition of the introduced herbivore community affected plant fitness directly via reducing plant biomass that predicted resource investment into reproductive organs (chapter 5). Interestingly, the herbivoreinduced changes in plant biomass were stronger than fitness consequences of herbivore-induced plant-mediated interactions with other community members (chapter 5). Fitness effects of indirect interactions often included a network of multiple organisms and could be mediated by plant traits. I show that all 
of these interactions involved effects of herbivores on pollinators that closely connected with the total number of seeds produced by a plant (chapter 5). For a species-rich community of phloem feeders as well as for the leaf-chewerrich community seed set was reduced via a chain of interactions that reduced pollinator visitation.

My work has unravelled that herbivore richness, functional traits and species identity alters direct and indirect interactions with other insects and has fitness effects on plants. This allows me to predict that selection on plant traits incorporates the effects of these interactions on plant fitness. However, due to the complexity and high connectivity of the interaction network it is very difficult to make predictions on directions of effects for plant-trait selection (Strauss, 2014). In many study systems diffuse evolution of plant traits has been reported, where selection on traits is not only explained by one-to-one species interactions, but rather is the effect of the network of interactions (Poelman \& Kessler, 2016; Strauss, 2014).

\section{CONCLUDING REMARKS AND FUTURE PERSPECTIVE}

In conclusion, my thesis provides answers to important questions on plant responses to multi-herbivore attack. I show that plants tailor induced defence strategies to deal with common patterns of sequential herbivore attack and anticipate arrival of the most prevalent herbivores. Additionally, I show that B. nigra plants channel their defence responses stronger towards a feeding-guild specific response when under multi-species attack by herbivores of the same feeding guild, but integrate responses when simultaneously confronted with a mix of herbivores from different feeding guilds. Importantly, under field conditions, I show that herbivore richness, functional trait and herbivore identity affect plant fitness and plant interactions with a herbivore, parasitoids and pollinators. Finally, I show that history of sequential attack is an important factor determining plant resistance to herbivores. 


\section{Integrating field-studies with mechanistic physiological and molecular biology}

In the last years we have been collecting knowledge on plant defences against stressors. All these studies, including my thesis, have helped us understanding some of the plant systems involved in stress detection, and the strategies to regulate an appropriated response. Nonetheless, how plants integrate information derived from multiple (i.e. more than two) stressors remains an open question. Moreover, how specific the induced plant phenotype is to the attackers remains to be further investigated. Future research should focus on unveiling the mechanisms that regulate plant responses to a combination of attackers. For such experiments a tight collaboration between ecologists and molecular biologists is needed. On the one hand, ecologists can provide valuable information on the type of interactions that happen in nature. On the other hand, molecular biologists can use this information to design ecologically meaningful experiments, where plants are challenged by stresses that they would experience in their natural environment. Investigating plant defence against their natural stressors is the only way that allows us inferring conclusions on the agents involved in plant-trait selection. These multi-disciplinary projects are a big -yet exciting- challenge that plant scientists should embrace.

\section{Climate change and insect decline: effect on plant trait selection}

In this thesis I have shown the importance of studying plant responses to multiple herbivores. First, I show that even plant physiological responses to multiple attack have some similarities with responses to single attack, important changes arise if more players are introduced. Second, I show that this is also the case for plant-mediated interactions between herbivores. A plant's ability to defend against herbivores is influenced by the history of multi-herbivore attack. Third, I have shown how multi-herbivore attack affects plant fitness and plant interactions with herbivores, parasitoids and pollinators. Given the current situation of global warming and insect extinction (Hallmann et al., 2020; Wagner et al., 2021), where many interactions between plants and insects 
are being altered, I predict that dramatic changes in plant-insect interactions are already happening. This change in interactions poses great challenges to ecologists and agricultural scientists. Undoubtedly, scientists will embrace the challenge and guide society to prevent ecological catastrophes (Harvey et al., 2020). In this context, research on plant interactions against multiple attackers can provide very relevant information. On one hand, it can help us predicting cascading effects on the plant-associated community, information that can be used for many purposes, including insect conservation strategies. On the other hand, as in agriculture crops are under the attack of multiple pests, designing of crop-protection practices can learn from fundamental studies on plant defence strategies to multi-herbivore attack.

ACKNOWLEDGEMENTS I thank Erik Poelman and Marcel Dicke for helpful comments on an earlier version of this chapter. 


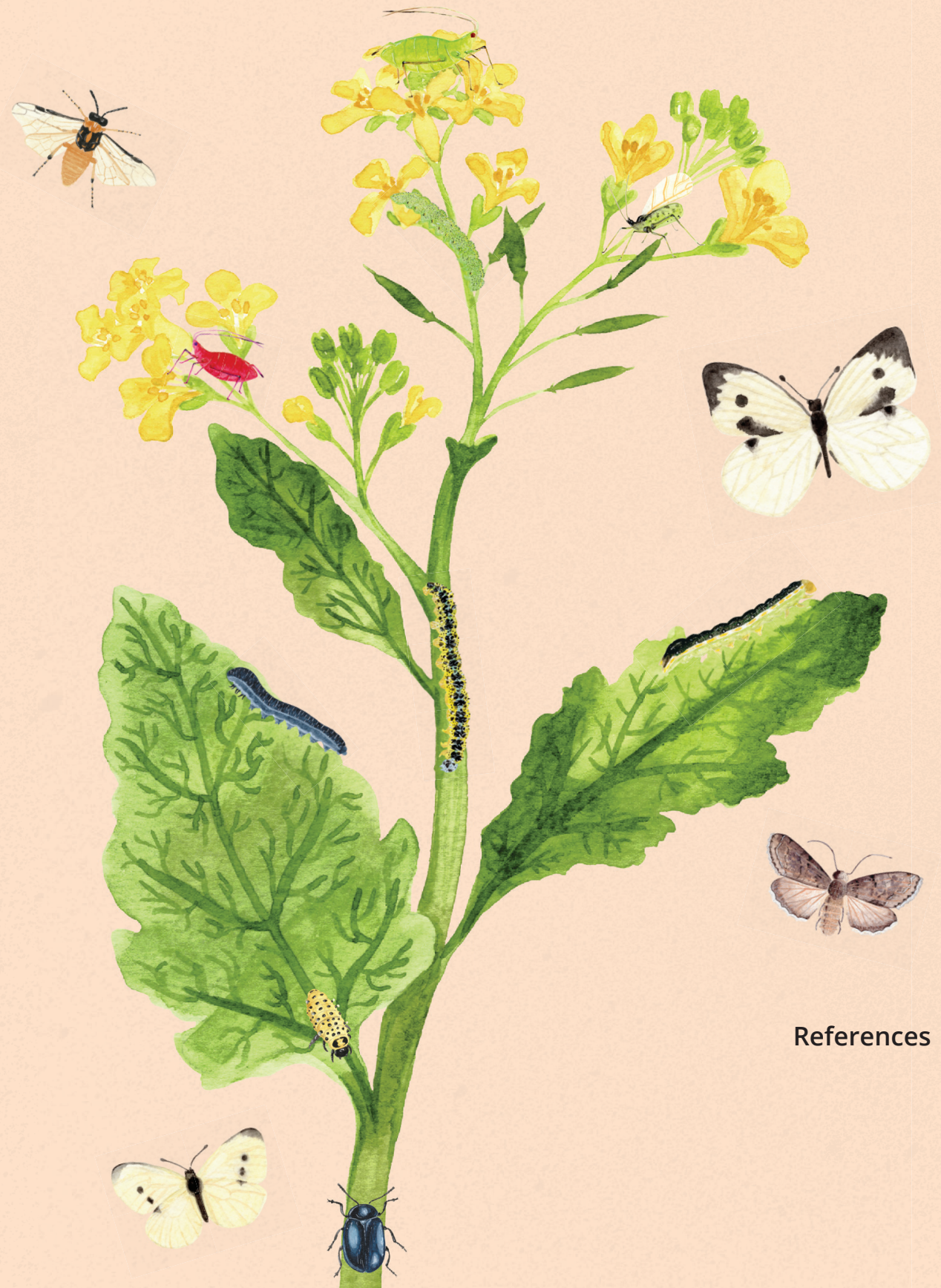

Aartsma, Y., Bianchi, F. J. J. A., van der Werf, W., Poelman, E. H., and Dicke, M. (2017). Herbivore-induced plant volatiles and tritrophic interactions across spatial scales. New Phytologist, 216(4), 1054-1063. https://doi.org/10.1111/nph.14475

Aartsma, Y., Cusumano, A., Fernández de Bobadilla, M., Rusman, Q., Vosteen, I., and Poelman, E. H. (2019). Understanding insect foraging in complex habitats by comparing trophic levels: insights from specialist hostparasitoid-hyperparasitoid systems. Current Opinion in Insect Science, 32, 54-60. https://doi.org/10.1016/j.cois.2018.11.001

Acevedo, F. E., Rivera-Vega, L. J., Chung, S. H., Ray, S., and Felton, G. W. (2015). Cues from chewing insects - the intersection of DAMPs, HAMPs, MAMPs and effectors. Current Opinion in Plant Biology, 26, 80-86. https://doi.org/10.1016/j.pbi.2015.05.029

Adio, A. M., Casteel, C. L., De Vos, M., Kim, J. H., Joshi, V., Li, B., Juéry, C., Daron, J., Kliebenstein, D. J., and Jander G. (2011). Biosynthesis and defensive function of $\mathrm{N}$-acetylornithine, a jasmonate-induced Arabidopsis metabolite. Plant Cell, 23(9), 3303-3318. https://doi.org/10.1105/ tpc.111.088989

Agrawal, A. A. (1999). Induced responses to herbivory in wild radish: Effects on several herbivores and plant fitness. Ecology, 80(5), 1713-1723. https://doi.org/10.1890/0012-9658(1999)080[1713:IRTHIW]2.0.CO;2

Agrawal, A. A. (2000). Specificity of induced resistance in wild radish: causes and consequences for two specialist and two generalist caterpillars. Oikos, 89(3), 493-500. https:// doi.org/10.1034/j.1600-0706.2000.890308.x

Agrawal, A. A. (2001). Phenotypic plasticity in the interactions and evolution of species. Science, 294(5541), 321-326. https://doi.org/10.1126/ science.1060701

Agrawal, A. A., Hastings, A. P., Patrick, E. T., and Knight, A. C. (2014). Specificity of herbivore-induced hormonal signaling and defensive traits in five closely related milkweeds (Asclepias spp.). Journal of Chemical Ecology, 40(7), 717-729. https:// doi.org/10.1007/s10886-014-0449-6 
Ahman, I., Kim, S. Y., and Zhu, L. H. (2019). Plant genes benefitting aphidspotential for exploitation in resistance breeding. Frontiers in Plant Science, 10, 14. https://doi.org/10.3389/fpls.2019.01452

Ali, J. G., and Agrawal, A. A. (2012) Specialist versus generalist insect herbivores and plant defense. Trends in Plant Science, 17(5), 293-302. https://doi.org/10.1016/j.tplants.2012.02.006

Ali, J. G., and Agrawal, A. A. (2014) Asymmetry of plant-mediated interactions between specialist aphids and caterpillars on two milkweeds. Functional Ecology, 28(6), 1404-1412. https://doi.org/10.1111/1365-2435.12271

Althaus, S. L., Berenbaum, M. R., Jordan, J., and Shalmon, D. A. (2021). No buzz for bees: Media coverage of pollinator decline. Proceedings of the National Academy of Sciences of the United States of America, 118(2), 1-8. https://doi.org/10.1073/pnas.2002552117

Ando, Y., Utsumi, S., and Ohgushi, T. (2017). Aphid as a network creator for the plant-associated arthropod community and its consequence for plant reproductive success. Functional Ecology, 31(3), 632-641. https://doi.org/10.1111/1365-2435.12780

Appel, H. M., and Cocroft, R. B. (2014). Plants respond to leaf vibrations caused by insect herbivore chewing. Oecologia, 175(4), 1257-1266. https://doi.org/10.1007/s00442-014-2995-6

Appel, H. M., Fescemyer, H., Ehlting, J., Weston, D., Rehrig, E., Joshi, T., Xu, D., Bohlmann, J., and Schultz, J. (2014). Transcriptional responses of Arabidopsis thaliana to chewing and sucking insect herbivores. Frontiers in Plant Science, 5, 585. https://doi.org/10.3389/fpls.2014.00565

Arena, G. D., Ramos-Gonzalez, P. L., Rogerio, L. A., Ribeiro-Alves, M., Casteel, C. L., Freitas-Astua, J., and Machado, M. A. (2018). Making a Better Home: Modulation of Plant Defensive Response by Brevipalpus Mites. Frontiers in Plant Science, 9(1147), 19. https://doi.org/10.3389/ fpls.2018.01147

Atkinson, N. J., and Urwin, P. E. (2012). The interaction of plant biotic and abiotic stresses: genes to the field. Journal of Experimental Botany, 63(10), 3523-3544. https:// doi.org/10.1093/jxb/err313 
Babikova, Z., Gilbert, L., Bruce, T. J. A., Birkett, M., Caulfield, J. C., Woodcock, C., Pickett, J. A., and Johnson, D. (2013). Underground signals carried through common mycelial networks warn neighbouring plants of aphid attack. Ecology Letters, 16(7), 835-843. https://doi.org/10.1111/ele.12115

Badri, D. V., Zolla, G., Bakker, M. G., Manter, D. K., and Vivanco, J. M. (2013). Potential impact of soil microbiomes on the leaf metabolome and on herbivore feeding behavior. New Phytologist, 198(1), 264-273. https://doi.org/10.1111/nph.12124

Ballaré, C. L., and Austin, A. T. (2019) Recalculating growth and defense strategies under competition: key roles of photoreceptors and jasmonates. Journal of Experimental Botany, 70(13), 3425-3434. https://doi.org/10.1093/jxb/erz237

Barbosa, P., Letourneau, D. K., and Agrawal A. A. (2012). (Eds.), Insect outbreaks revisited. Oxford: Wiley-Blackwell. https:// doi. org $/ 10.1002 / 9781118295205$

Barton, K. E., and Koricheva, J. (2010) The ontogeny of plant defense and herbivory: Characterizing general patterns using meta-Analysis. The American Naturalist, 175(4), 481-493. https://doi.org/10.1086/650722

Bates, D., Maechler, M., Bolker, B., and Walker, S. (2015). Fitting linear mixedeffects models using lme4. Journal of Statistical Software, 67, 1-48. https://doi.org/10.18637/jss.v067.i01

Bekaert, M., Edger, P. P., Hudson, C. M., Pires, J. C., and Conant, G. C. (2012). Metabolic and evolutionary costs of herbivory defense: Systems biology of glucosinolate synthesis. New Phytologist, 196(2), 596-605. https://doi.org/10.1111/j.1469-8137.2012.04302.x

Bell, E., Creelman, R. A., and Mullet, J. E. (1995). A chloroplast lipoxygenase is required for wound-induced jasmonic acid accumulation in Arabidopsis. Proceedings of the National Academy of Sciences of the United States of America, 92(19), 8675-8679. https:// doi.org/10.1073/pnas.92.19.8675

Berg, G. (2009). Plant-microbe interactions promoting plant growth and health: perspectives for controlled use of microorganisms in agriculture. Applied Microbiology and Biotechnology, 84(1), 11-18. https://doi.org/10.1007/ s00253-009-2092-7 
Bidart-Bouzat, M. G., and Kliebenstein, D. (2011). An ecological genomic approach challenging the paradigm of differential plant responses to specialist versus generalist insect herbivores. Oecologia, 167(3), 677-689. https://doi.org/10.1007/s00442-011-2015-z

Bischoff, A., and Trémulot, S. (2011). Differentiation and adaptation in Brassica nigra populations: Interactions with related herbivores. Oecologia, 165, 971-981. https:// doi.org/10.1007/s00442-010-1798-7

Blubaugh, C. K., Asplund, J. S., Eigenbrode, S. D., Morra, M. J., Philips, C. R., Popova, I. E., Reganold, J. P., and Snyder, W. E. (2018). Dual-guild herbivory disrupts predator-prey interactions in the field. Ecology, 99(5), 1089-1098. https://doi.org/10.1002/ecy.2192

Body, M. J. A., Neer, W. C., Vore, C., Lin, C. H., Vu, D. C., Schultz, J. C., Cocroft, R. B., and Appel, H. M. (2019). Caterpillar chewing vibrations cause changes in plant hormones and volatile emissions in Arabidopsis thaliana. Frontiers in Plant Science, 10, 810. https://doi.org/10.3389/ fpls.2019.00810

Bonnet, C., Lassueur, S., Ponzio, C., Gols, R., Dicke, M., and Reymond, P. (2017). Combined biotic stresses trigger similar transcriptomic responses but contrasting resistance against a chewing herbivore in Brassica nigra. BMC Plant Biology, 17(1), 127. https://doi.org/10.1186/s12870-017-1074-7

Broekgaarden, C., Voorrips, R. E., Dicke, M., and Vosman, B. (2011). Transcriptional responses of Brassica nigra to feeding by specialist insects of different feeding guilds. Insect Science, 18(3), 259-272. https://doi. org/10.1111/j.1744-7917.2010.01368.x

Bruessow, F., Gouhier-Darimont, C., Buchala, A., Metraux, J. P., and Reymond, P. (2010). Insect eggs suppress plant defence against chewing herbivores. Plant Journal, 62(5), 876-885. https:// doi.org/10.1111/j.1365313X.2010.04200.x

Bukovinszky, T., Poelman, E. H., Kamp, A., Hemerik, L., Prekatsakis, G., and Dicke, M. (2012). Plants under multiple herbivory: Consequences for parasitoid search behaviour and foraging efficiency. Animal Behaviour, 83(2), 501-509. https:// doi.org/10.1016/j.anbehav.2011.11.027 
Bukovinszky, T., Tréfás, H., Van Lenteren, J. C., Vet, L. E. M., and Fremont, J. (2004). Plant competition in pest-suppressive intercropping systems complicates evaluation of herbivore responses. Agriculture, Ecosystems and Environment, 102(2), 185-196. https://doi.org/10.1016/j. agee.2003.08.008

Bustos-Segura, C., Cuny, M. A. C., and Benrey, B. (2020). Parasitoids of leaf herbivores enhance plant fitness and do not alter caterpillar-induced resistance against seed beetles. Functional Ecology, 34(3), 586-596. https://doi.org/10.1111/1365-2435.13478

Caarls, L., Pieterse, C. M. J., and Van Wees, S. (2015) How salicylic acid takes transcriptional control over jasmonic acid signaling. Frontiers in Plant Science, 6, 170. https:// doi.org/10.3389/fpls.2015.00170

Chandran, D., Inada, N., Hather, G., Kleindt, C. K., and Wildermuth, M. C. (2010). Laser microdissection of Arabidopsis cells at the powdery mildew infection site reveals site-specific processes and regulators. Proceedings of the National Academy of Sciences of the United States of America, 107(1), 460-465. https:// doi.org/10.1073/pnas.0912492107

Chen, G., Kim, H. K., Klinkhamer, P. G., and Escobar-Bravo, R. (2020). Sitedependent induction of jasmonic acid-associated chemical defenses against western flower thrips in Chrysanthemum. Planta, 251(1), 8. https:// doi.org/10.1007/s00425-019-03292-2

Chidawanyika, F., Mudavanhu, P., and Nyamukondiwa, C. (2019). Global climate change as a driver of bottom-up and top-down factors in agricultural landscapes and the fate of host-parasitoid interactions. Frontiers in Ecology and Evolution, 80(7), 80. https://doi.org/10.3389/fevo.2019.00080

Chrétien, L. T. S., David, A., Daikou, E., Boland, W., Gershenzon, J., Giron, D., Dicke, M., and Lucas-Barbosa, D. (2018). Caterpillars induce jasmonates in flowers and alter plant responses to a second attacker. New Phytologist, 217(3), 1279-1291. https://doi.org/10.1111/nph.14904

Chrétien, L. T. S., van der Heide, H., Greenberg, L. O., Giron, D., Dicke, M., and Lucas-Barbosa, D. (2021). Multiple attack to inflorescences of an annual plant does not interfere with the attraction of 
parasitoids and pollinators. Journal of Chemical Ecology, 47, 175-191. https:// doi.org/10.1007/s10886-020-01239-6

Connell, J. H. (1980). Diversity and the coevolution of competitors, or the ghost of competition past. Oikos, 35(2), 131-138. https://doi. org $/ 10.2307 / 3544421$

Conrath, U., Beckers, G. J. M., Flors, V., García-Agustín, P., Jakab, G., Mauch, F., Newman, M. A., Pieterse, C. M. J., Poinssot, B., Pozo, M. J., Pugin, A., Schaffrath, U., Ton, J., Wendehenne, D., Zimmerli, L., and MauchMani, B. (2006). Priming: getting ready for battle. Molecular Pant-Microbe Interactions, 19(10), 1062-1071. https://doi.org/10.1094/MPMI-19-1062 Coolen, S., Proietti, S., Hickman, R., Davila Olivas, N. H., Huang, P. P., Van Verk, M. C., Van Pelt, J. A., Wittenberg, A. H. J., De Vos, M., Prins, M., Van Loon, J. J. A., Aarts, M. G. M., Dicke, M., Pieterse, C. M. J., and Van Wees, S. C. M. (2016). Transcriptome dynamics of Arabidopsis during sequential biotic and abiotic stresses. The Plant Journal, 86(3), 249-267. https:// doi.org/10.1111/tpj.13167

Cozzolino, S., Fineschi, S., Litto, M., Scopece, G., Trunschke, J., and Schiestl, F. P. (2015). Herbivory increases fruit set in Silene latifolia: A consequence of induced pollinator-attracting floral volatiles? Journal of Chemical Ecology, 41(7), 622-630. https://doi.org/10.1007/s10886-015-0597-3

Dalin, P., and Björkman, C. (2003). Adult beetle grazing induces willow trichome defence against subsequent larval feeding. Oecologia, 134(1), 112-118. https:// doi.org/10.1007/s00442-002-1093-3

Davidson-Lowe, E., Szendrei, Z., and Ali, J. G. (2019). Asymmetric effects of a leaf-chewing herbivore on aphid population growth. Ecological Entomology, 44(1), 81-92. https:// doi.org/10.1111/een.12681

Davila Olivas, N. H., Coolen, S., Huang, P., Severing, E., van Verk, M. C., Hickman, R., Wittenberg, A. H. J., de Vos, M., Prins, M., van Loon, J. J. A., Aarts, M. G. M., van Wees, S. C. M., Pieterse, C. M. J., and Dicke, M. (2016). Effect of prior drought and pathogen stress on Arabidopsis transcriptome changes to caterpillar herbivory. New Phytologist, 210(4), 1344-1356. https://doi.org/10.1111/nph.13847 
Davila Olivas, N. H., Frago, E., Thoen, M. P. M., Kloth, K. J., Becker, F. F. M., van Loon, J. J. A., Gort, G., Keurentjes, J. J. B., van Heerwaarden, J., and Dicke, M. (2017). Natural variation in life history strategy of Arabidopsis thaliana determines stress responses to drought and insects of different feeding guilds. Molecular Ecology, 26(11), 2959-2977. https:// doi.org/10.1111/mec.14100

Davila Olivas, N. H., Kruijer, W., Gort, G., Wijnen, C. L., van Loon, J. J. A., and Dicke, M. (2017). Genome-wide association analysis reveals distinct genetic architectures for single and combined stress responses in Arabidopsis thaliana. New Phytologist, 213(2), 838-851. https://doi.org/10.1111/nph.14165

De Boer, J. G., Hordijk, C. A., Posthumus, M. A., and Dicke, M. (2008). Prey and non-prey arthropods sharing a host plant: Effects on induced volatile emission and predator attraction. Journal of Chemical Ecology, 34(3), 281290. https://doi.org/10.1007/s10886-007-9405-z

De Rijk, M., Dicke, M., and Poelman, E. H. (2013). Foraging behaviour by parasitoids in multiherbivore communities. Animal Behaviour, 85(6), 1517-1528. https://doi.org/10.1016/j.anbehav.2013.03.034

De Rijk, M., Krijn, M., Jenniskens, W., Engel, B., Dicke, M., and Poelman, E. H. (2016). Flexible parasitoid behaviour overcomes constraint resulting from position of host and nonhost herbivores. Animal Behaviour, 113, 125-135. https://doi.org/10.1016/j.anbehav.2016.01.001

De Rijk, M., Yang, D., Engel, B., Dicke, M., and Poelman, E. H. (2016). Feeding guild of non-host community members affects host-foraging efficiency of a parasitic wasp. Ecology, 97(6), 1388-1399. https://doi.org/10.1890/151300.1 .

De Rijk, M., Wang, Q., Papagiannaki, E., Dicke, M., and Poelman, E. H. (2016). Herbivore species identity rather than diversity of the nonhost community determines foraging behaviour of the parasitoid wasp Cotesia glomerata. Entomologia Experimentalis et Applicata, 161(1), 20-30. https://doi.org/10.1111/eea.12493

De Rijk, M., Zhang, X., Van Der Loo, J. A. H., Engel, B., Dicke, M., and Poelman, E. H. (2016). Density-mediated indirect interactions alter host foraging 
behaviour of parasitoids without altering foraging efficiency. Ecological Entomology, 41(5), 562-571. https://doi.org/10.1111/een.12325

de Vries, J., Evers, J. B., Dicke, M., and Poelman, E. H. (2019). Ecological interactions shape the adaptive value of plant defence: Herbivore attack versus competition for light. Functional Ecology, 33(1), 129-138. https:// doi.org/10.1111/1365-2435.13234.

de Vries, J., Evers, J. B., and Poelman, E. H. (2017). Dynamic Plant-Plant-Herbivore Interactions Govern Plant Growth-Defence Integration. Trends in Plant Science, 22(4), 329-337. https:// doi.org/10.1016/j.tplants.2016.12.006

de Vries, J., Poelman, E. H., Anten, N., and Evers, J. B. (2018). Elucidating the interaction between light competition and herbivore feeding patterns using functional-structural plant modelling. Annals of Botany, 121(5), 1019-1031. https:// doi.org/10.1093/aob/mcx212

Delphia, C. M., Mescher, M. C., and De Moraes, C. M. (2007). Induction of plant volatiles by herbivores with different feeding habits and the effects of induced defenses on host-plant selection by thrips. Journal of Chemical Ecology, 33(5), 997-1012. https://doi.org/10.1007/s10886-007-9273-6

Desurmont, G. A., Xu, H., and Turlings, T. C. J. (2016). Powdery mildew suppresses herbivore-induced plant volatiles and interferes with parasitoid attraction in Brassica rapa. Plant Cell and Environment, 39(9), 1920-1927. https:// doi.org/10.1111/pce.12752

Devegili, A. M., and Chalcoff, V. R. (2020). Nectar shortage caused by aphids may reduce seed output via pollination interference. Oecologia, 194(3), 321-332. https://doi.org/10.1007/s00442-020-04712-x

Dicke, M., and Baldwin, I. T. (2010). The evolutionary context for herbivoreinduced plant volatiles: beyond the "cry for help." Trends in Plant Science, 15(3), 167-175. https://doi.org/10.1016/j.tplants.2009.12.002

Dicke, M., Van Loon, J. J. A., and Soler, R. (2009). Chemical complexity of volatiles from plants induced by multiple attack. Nature Chemical Biology, 5(5), 317-324. https://doi.org/10.1038/nchembio.169

Eberl, F., Fernandez de Bobadilla, M., Reichelt, M., Hammerbacher, A., Gershenzon, J., and Unsicker, S. B. (2020). Herbivory meets fungivory: 
insect herbivores feed on plant pathogenic fungi for their own benefit. Ecology Letters, 23(7), 1073-1084. https://doi.org/10.1111/ele.13506

Eberl, F., Hammerbacher, A., Gershenzon, J., and Unsicker, S. B. (2018). Leaf rust infection reduces herbivore-induced volatile emission in black poplar and attracts a generalist herbivore. New Phytologist, 220(3), 760772. https://doi.org/10.1111/nph.14565

Ehrlich, P. R., and Raven, P. H. (1964). Butterflies and plants - A study in coevolution. Evolution, 18, 586-608. https://doi.org/10.2307/2406212

Eisenring, M., Glauser, G., Meissle, M., and Romeis, J. (2018) Differential impact of herbivores from three feeding guilds on systemic secondary metabolite induction, phytohormone levels and plant-mediated herbivore interactions. Journal of Chemical Ecology, 44, 1178-1189. https://doi.org/10.1007/s10886-018-1015-4

El-Sayed, A. M., Knight, A. L., Byers, J. A., Judd, G. J. R., and Suckling, D. M. (2016). Caterpillar-induced plant volatiles attract conspecific adults in nature. Scientific Reports, 6(May), 1-14. https://doi.org/10.1038/srep37555

Elzinga, D. A., De Vos, M., and Jander, G. (2014). Suppression of Plant Defenses by a Myzus persicae (Green Peach Aphid) Salivary Effector Protein. Molecular Plant-Microbe Interactions, 27(7), 747-756. https:// doi.org/10.1094/MPMI-01-14-0018-R

Elzinga, D. A., and Jander, G. (2013). The role of protein effectors in plantaphid interactions. Current Opinion in Plant Biology, 16(4), 451-456. https://doi.org/10.1016/j.pbi.2013.06.018

Erb, M. (2018). Plant defenses against herbivory: Closing the fitness gap. Trends in Plant Science, 23(3), 187-194. https://doi.org/10.1016/j. tplants.2017.11.005

Erb, M., Meldau, S., and Howe, G. A. (2012). Role of phytohormones in insect-specific plant reactions. Trends in Plant Science, 17(5), 250-259. https://doi.org/10.1016/j.tplants.2012.01.003

Erb, M., and Reymond, P. (2019). Molecular Interactions between Plants and Insect Herbivores. Annual Review of Plant Biology, 70, 527-557. https:// doi.org/10.1146/annurev-arplant-050718-095910 
Erb, M., Robert, C. A. M., Hibbard, B. E., and Turlings, T. C. J. (2011). Sequence of arrival determines plant-mediated interactions between herbivores. Journal of Ecology, 99(1), 7-15. https://doi.org/10.1111/j.13652745.2010.01757.x

Farmer, E. E., and Ryan, C. A. (1990). Interplant communication: Airborne methyl jasmonate induces synthesis of proteinase inhibitors in plant leaves. Proceedings of the National Academy of Sciences of the United States of America, 87(19), 7713-7716. https://doi.org/10.1073/pnas.87.19.7713

Fatouros, N. E., Paniagua Voirol, L. R., Drizou, F., Doan, Q. T., Pineda, A., Frago, E., and van Loon, J. J. A. (2015). Role of Large Cabbage White butterfly male-derived compounds in elicitation of direct and indirect egg-killing defenses in the black mustard. Frontiers in Plant Science, 6, 794. https:// doi.org/10.3389/fpls.2015.00794

Fernández de Bobadilla, M., Bourne, M. E., Bloem, J., Kalisvaart, S. N., Gort, G., Dicke, M., and Poelman, E. H. (2021). Insect species richness affects plant responses to multi-herbivore attack. New Phytologist. https://doi. org/10.1111/nph.17228

Franco, F. P., Moura, D. S., Vivanco, J. M., and Silva-Filho, M. C. (2017). Plantinsect-pathogen interactions: a naturally complex ménage à trois. Current Opinion in Microbiology, 37(June), 54-60. https://doi.org/10.1016/j. mib.2017.04.007

Gervasi, D. D. L., and Schiestl, F. P. (2017). Real-time divergent evolution in plants driven by pollinators. Nature Communications, 8, 1-8. https://doi. org $/ 10.1038 /$ ncomms14691

Giordanengo, P., Brunissen, L., Rusterucci, C., Vincent, C., van Bel, A., Dinant, S., Girousse, C., Faucher, M., and Bonnemain, J. L. (2010). Compatible plantaphid interactions: How aphids manipulate plant responses. Comptes Rendus Biologies, 333(6-7), 516-523. https://doi.org/10.1016/j.crvi.2010.03.007

Giron, D., Dubreuil, G., Bennett, A., Dedeine, F., Dicke, M., Dyer, L. A., Erb, M., Harris, M. O., Huguet, E., Kaloshian, I., et al. (2018). Promises and challenges in insect-plant interactions. Entomologia Experimentalis et Applicata, 166(5), 319-343. https://doi.org/10.1111/eea.12679 
Glaum, P., and Kessler, A. (2017). Functional reduction in pollination through herbivore-induced pollinator limitation and its potential in mutualist communities. Nature Communications, 8(1), 2031. https://doi.org/10.1038/s41467-017-02072-4

Gonzales-Vigil, E., Bianchetti, C. M., Phillips Jr., G. N., and Howe, G. A. (2011). Adaptive evolution of threonine deaminase in plant defense against insect herbivores. Proceedings of the National Academy of Sciences of the United States of America, 108(14), 5897-5902. https://doi.org/10.1073/pnas.1016157108

Gouhier-Darimont, C., Stahl, E., Glauser, G., and Reymond, P. (2019). The Arabidopsis lectin receptor kinase lecrk-i.8 is involved in insect egg perception. Frontiers in Plant Science, 10, 623. https://doi.org/10.3389/ fpls.2019.00623

Grass, I., Bohle, V., Tscharntke, T., and Westphal, C. (2018). How plant reproductive success is determined by the interplay of antagonists and mutualists. Ecosphere, 9(2), e02106. https://doi.org/10.1002/ecs2.2106

Guo, Z., Dolll, K., Dastjerdi, R., Karlovsky, P., Dehne, H. W., and Altincicek, B. (2014). Effect of fungal colonization of wheat grains with Fusarium spp. on food choice, weight gain and mortality of meal beetle larvae (Tenebrio molitor). PLoS ONE, 9(6), e100112. https://doi.org/10.1371/ journal.pone.0100112

Hallmann, C. A., Sorg, M., Jongejans, E., Siepel, H., Hofland, N., Schwan, H., Stenmans, W., Müller, A., Sumser, H., Hörren, T., Goulson, D., and De Kroon, H. (2017). More than 75 percent decline over 27 years in total flying insect biomass in protected areas. PLoS ONE, 12(10), e0185809. https://doi.org/10.1371/journal.pone.0185809

Hallmann, C. A., Ssymank, A., Sorg, M., De Kroon, H., and Jongejans, E. (2020). Insect biomass decline scaled to species diversity: general patterns derived from a hoverfly community. Proceedings of the National Academy of Sciences, 118(2), 1-8. https://doi.org/10.1073/pnas.2002554117

Halsch, C., Shapiro, A., Fordyce, J., Nice, C., Thorne, J., Waetjen, D., and Forister, M. (2020). Insects and recent climate change. Proceedings of the National Academy of Sciences, 118(2), 1-9. https://doi.org/10.1101/2020.03.09.984328 
Harvey, J. A., Heinen, R., Armbrecht, I., Basset, Y., Baxter-Gilbert, J. H., Bezemer, T. M., Böhm, M., Bommarco, R., Borges, P. A. V., Cardoso, P., Clausnitzer, V., Cornelisse, T., Crone, E. E., Dicke, M., Dijkstra, K. D. B., Dyer, L., Ellers, J., Fartmann, T., Forister, M. L., Furlong, M. J., Garcia-Aguayo, A., Gerlach, J., Gols, R., Goulson, D., Habel, J.-C., Haddad, N. M., Hallmann, C. A., Henriques, S., Herberstein, M. E., Hochkirch, A., Hughes A. C., Jepsen, S., Jones, T. H., Kaydan, B. M., Kleijn, D., Klein, A.-M., Latty, T., Leather, S. R., Lewis, S. M., Lister, B. C., Losey, J. E., Lowe, E. C., Macadam, C. R., Montoya-Lerma, J., Nagano, C. D., Ogan, S., Orr, M. C., Painting, C. J., Pham, T.-H., Potts, S. G., Rauf, A., Roslin, T. L., Samways, M. J., Sanchez-Bayo, F., Sar, S. A., Schultz, C. B., Soares, A. O., Thancharoen, A., Tscharntke, T., Tylianakis, J. M., Umbers, K. D. L., Vet, L. E. M., Visser, M. E., Vujic, A., Wagner, D. L., Wallis De Vries, M. F., Westphal, C., White, T. E., Wilkins, V. L., Williams, P. H., Wyckhuys, K. A. G., Zhu, Z.-R., and de Kroon, H. (2020). International scientists formulate a roadmap for insect conservation and recovery. Nature Ecology and Evolution, 4(2), 174-176. https://doi.org/10.1038/s41559-019-1079-8

Hauser, T. P., Christensen, S., Heimes, C., and Kixr, L. P. (2013). Combined effects of arthropod herbivores and phytopathogens on plant performance. Functional Ecology, 27(3), 623-632. https://doi.org/10.1111/13652435.12053

Hedges, L. V., Gurevitch, J., and Curtis, P. S. (1999). The meta-analysis of response ratios in experimental ecology. Ecology, 80(4), 1150-1156. https://doi.org/10.2307/177062

Heidel, A. J., and Baldwin, I. T. (2004). Microarray analysis of salicylic acid- and jasmonic acid-signalling in responses of Nicotiana attenuata to attack by insects from multiple feeding guilds. Plant Cell and Environment, 27(11), 1362-1373. https:// doi.org/10.1111/j.1365-3040.2004.01228.x

Heil, M. (2010). Plastic defence expression in plants. Evolutionary Ecology, 24(3), 555-569. https:// doi.org/10.1007/s10682-009-9348-7

Herrera, C. M., Medrano, M., Rey, P. J., Sánchez-Lafuente, A. M., Garcia, M. B., Guitián, J., and Manzaneda, A. J. (2002). Interaction of pollinators and herbivores on plant fitness suggests a pathway for correlated evolution 
of mutualism- and antagonism-related traits. Proceedings of the National Academy of Sciences of the United States of America, 99(26), 16823-16828. https:// doi.org/10.1073/pnas.252362799

Hervé, M. R., and Erb, M. (2019). Distinct defense strategies allow different grassland species to cope with root herbivore attack. Oecologia, 191(1), 127-139. https:// doi.org/10.1007/s00442-019-04479-w

Hickman, R., Van Verk, M. C., Van Dijken, A. J. H., Pereira Mendes, M., VroegopVos, I. A., Caarls, L., Steenbergen, M., Van der Nagel, I., Wesselink, G. J., Jironkin, A., Talbot, A., Rhodes, J., De Vries, M., Schuurink, R. C., Denby, K., Pieterse, C. M. J., and Van Wees, S. C. M. (2017). Architecture and dynamics of the jasmonic acid gene regulatory network. The Plant Cell, 29(9), 20862105. https://doi.org/10.1105/tpc.16.00958lant Cell 29(9), 2086-2105.

Hilker, M., and Fatouros, N. E. (2015). Plant responses to insect egg deposition. Annual Review of Entomology, 60, 493-515. https://doi.org/10.1146/ annurev-ento-010814-020620

Hopkins, R. J., van Dam, N. M., and van Loon, J. J. A. (2009). Role of glucosinolates in insect-plant relationships and multitrophic interactions. Annual Review of Entomology, 54, 57-83. https://doi. org/10.1146/annurev.ento.54.110807.090623

Hothorn, T., Bretz, F., and Westfall, P. (2008). Simultaneous inference in general parametric models. Biometrical Journal, 50(3), 346-363. https://doi.org/10.1002/bimj.200810425

Jakobs, R., Schweiger, R., and Müller, C. (2018). Aphid infestation leads to plant part-specific changes in phloem sap chemistry, which may indicate niche construction. New Phytologist, 221(1), 503-514. https://doi.org/10.1111/ nph.15335

Jaouannet, M., Rodriguez, P. A., Thorpe, P., Lenoir, C. J. G., MacLeod, R., Escudero-Martinez, C., and Bos, J. I. B. (2014). Plant immunity in plant-aphid interactions. Frontiers in Plant Science, 5, 663. https://doi. org/10.3389/fpls.2014.00663

Jiang Y., Chuan-Xi, Z., Chenf R., and He S. Y. (2019). Challenging battles of plants with phloem-feeding insects and prokaryotic pathogens. Proceedings 
of the National Academy of Sciences of the United States of America, 116(47), 23390-23397. https://doi.org/10.1073/pnas.1915396116

Johnson, J. B., and Omland, K. S. (2004). Model selection in ecology and evolution. Trends in Ecology and Evoution, 19(2), 101-108. https://doi.org/10.1016/j.tree.2003.10.013

Johnson, S. N., Rowe, R. C., and Hall, C. R. (2020). Aphid feeding induces phytohormonal cross-talk without affecting silicon defense against subsequent chewing herbivores. Plants, 9(8), 1009. https:// doi. org $/ 10.3390 /$ plants9081009

Jones, J. D. G., and Dangl, J. L. (2006). The plant immune system. Nature, 444(7117), 323-329. https:// doi.org/10.1038/nature05286

Karban, R. (2011). The ecology and evolution of induced resistance against herbivores. Functional Ecology, 25(2), 339-347. https://doi.org/10.1111/j.1365-2435.2010.01789.x

Karban, R. (2019). The ecology and evolution of induced responses to herbivory and how plants perceive risk. Ecological Entomology, 45(1), 1-9. https:// doi.org/10.1111/een.12771

Karban, R., Agrawal, A. A., and Mangel, M. (1997). The benefits of induced defenses against herbivores. Ecology, 78(5), 1351-1355. https://doi. org/10.1890/0012-9658(1997)078[1351:TBOIDA]2.0.CO;2

Keith, R. A., and Mitchell-Olds, T. (2017). Testing the optimal defense hypothesis in nature: Variation for glucosinolate profiles within plants. PLOS ONE, 12(7), e0180971. https://doi.org/10.1371/journal.pone.0180971

Kessler, A., and Baldwin, I. T. (2004). Herbivore-induced plant vaccination. Part I. The orchestration of plant defenses in nature and their fitness consequences in the wild tobacco Nicotiana attenuata. Plant Journal, 38(4), 639-649. https://doi.org/10.1111/j.1365-313X.2004.02076.x

Kessler, A., and Chautá, A. (2020). The ecological consequences of herbivoreinduced plant responses on plant-pollinator interactions. Emerging Topics in Life Sciences, 4(1), 33-43. https:// doi.org/10.1042/ETLS20190121

Kiełkiewicz, M., Barczak-Brzyżek, A., Karpińska, B., and Filipecki, M. (2019). Unravelling the complexity of plant defense induced by a simultaneous 
and sequential mite and aphid infestation. International Journal of Molecular Sciences, 20(4), 806. https:// doi.org/10.3390/ijms20040806

Kliebenstein, D. J. (2014). Orchestration of plant defense systems: Genes to populations. Trends in Plant Science, 19(4), 250-255. https://doi. org/10.1016/j.tplants.2014.01.003

Koornneef, A., and Pieterse, C. M. J. (2008). Cross talk in defense signaling. Plant Physology, 146(3), 839-844. https://doi.org/10.1104/pp.107.112029

Kroes, A., Broekgaarden, C., Castellanos Uribe, M., May, S., van Loon, J. J. A., and Dicke, M. (2017). Brevicoryne brassicae aphids interfere with transcriptome responses of Arabidopsis thaliana to feeding by Plutella xylostella caterpillars in a density-dependent manner. Oecologia, 183(1), 107-120. https://doi.org/10.1007/s00442-016-3758-3

Kroes, A., Stam, J. M., David, A., Boland, W., van Loon, J. J. A., Dicke, M., and Poelman, E. H. (2016). Plant-mediated interactions between two herbivores differentially affect a subsequently arriving third herbivore in populations of wild cabbage. Plant Biology, 18(6), 981-991. https://doi. org/10.1111/plb.12490

Kroes, A., Van Loon, J. J. A., and Dicke, M. (2015). Density-dependent interference of aphids with caterpillar-induced defenses in Arabidopsis: Involvement of phytohormones and transcription factors. Plant and Cell Physiology, 56(1), 98-106. https://doi.org/10.1093/pcp/pcu150

Labandeira, C. C., Johnson, K. R., and Wilf, P. (2002). Impact of the terminal Cretaceous event on plant-insect associations. Proceedings of the National Academy of Sciences of the United States of America, 99, 2061-2066. https:// doi.org/10.1073/pnas.042492999

Lajeunesse, M. J. (2011). On the meta-analysis of response ratios for studies with correlated and multi-group designs. Ecology, 92(11), 2049-2055. https:// doi.org/10.1890/11-0423.1

Lankau, R. A., and Strauss, S. Y. (2008). Community complexity drives patterns of natural selection on a chemical defense of Brassica nigra. American Naturalist, 171(2), 150-161. https://doi.org/10.1086/524959 
Lenth, R. (2016). Least-Squares Means: The R Package 1smeans. Journal of Statistical Software. 69(1), 1-33. https://doi.org/10.18637/jss.v069.i01

Lenth, R. (2018). emmean: Estimated Marginal Means, aka Least-Squares Means. R Package Version 1.2.3. Available at: https://CRAN.R-project.org/ package $=$ emmeans .

Leon-Reyes, A., Van der Does, D., De Lange, E. S., Delker, C. Wasternack, C., Van Wees, S. C. M., Ritsema, T., and Pieterse, C. M. (2010). Salicylate-mediated suppression of jasmonate-responsive gene expression in Arabidopsis is targeted downstream of the jasmonate biosynthesis pathway. Planta, 232, 1423-1432. https://doi.org/10.1007/s00425-010-1265-z

Li, B., Förster, C., Robert, C. A. M., Züst, T., Hu, L., Machado, R. A. R., Berset, J.-D., Handrick, V., Knauer, T., Hensel, G., Chen, W., Kumlehn, J., Yang, P., Keller, B., Gershenzon, J., Jander, G., Köllner, T. G., and Erb, M. (2018). Convergent evolution of a metabolic switch between aphid and caterpillar resistance in cereals. Science Advances, 4(12), eaat6797. https:// doi.org/10.1126/sciadv.aat6797

Li, J., Brader, G., and Palva, E. T. (2004). The WRKY70 transcription factor: A node of convergence for jasmonate-mediated and salicylate-mediated signals in plant defense. Plant Cell, 16(2), 319-331. https://doi.org /10.1105/tpc.016980

Li, R., Zhang, J., Zhou, G., Wang, Q., Bian, W., Erb, M., and Lou, Y. (2015). Prioritizing plant defence over growth through WRKY regulation facilitates infestation by non-target herbivores. Elife, 4, e04805. https:// doi.org/10.7554/eLife.04805

Li, Y. H., Stam, J. M., Poelman, E. H., Dicke, M., and Gols, R. (2016). Community structure and abundance of insects in response to early-season aphid infestation in wild cabbage populations. Ecological Entomology, 41(4), 378388. https://doi.org/10.1111/een.12308

Li, Y. H., Dicke, M., Kroes, A., Liu, W., and Gols, R. (2016). Interactive effects of cabbage aphid and caterpillar herbivory on transcription of plant genes associated with phytohormonal signalling in wild cabbage. Journal of Chemical Ecology, 42(8), 793-805. https://doi.org/10.1007/s10886-016-0738-3 
Loreau, A., and Hector, A. (2011). Partitioning selection and complementarity in biodiversity experiments. Nature, 412(6842), 72-76. https://doi.org /10.1038/35083573

Lucas-Barbosa, D. (2016). Integrating Studies on Plant-Pollinator and PlantHerbivore Interactions. Trends in Plant Science, 21(2), 125-133. https:// doi.org/10.1016/j.tplants.2015.10.013

Lucas-Barbosa, D., Sun, P., Hakman, A., van Beek, T. A., van Loon, J. J. A., and Dicke, M. (2016). Visual and odour cues: Plant responses to pollination and herbivory affect the behaviour of flower visitors. Functional Ecology, 30(3), 431-441. https:// doi.org/10.1111/1365-2435.12509

Maffei, M. E., Mithöfer, A., and Boland, W. (2007). Before gene expression: early events in plant-insect interaction. Trends in Plant Science, 12(7), 310 316. https:// doi.org/10.1016/j.tplants.2007.06.001

Martinez-Medina, A., Flors, V., Heil, M., Mauch-Mani, B., Pieterse, C. M. J., Pozo, M. J., Ton, J., van Dam, N. M., and Conrath, U. (2016). Recognizing Plant Defense Priming. Trends in Plant Science, 21 (10), 818-822. https://doi.org/10.1016/j.tplants.2016.07.009

Mathur, V., Tytgat, T. O. G., de Graaf, R. M., Kalia, V., Sankara Reddy, A., Vet, L. E. M., and van Dam, N. M. (2013). Dealing with double trouble: Consequences of single and double herbivory in Brassica juncea. Chemoecology, 23(2), 71-82. http:// dx.doi.org/10.1007/s00049-0120120-z

McArt, S. H., Halitschke, R., Salminen, J.-P., and Thaler, J. S. (2013). Leaf herbivory increases plant fitness via induced resistance to seed predators. Ecology, 94(4), 966-975. https://doi.org/10.1890/12-1664.1

Mertens, D., Boege, K., Kessler, A., Koricheva, J., Thaler J. S., Whiteman, N. K., and Poelman, E.H. (2021). Predictability of biotic stress structures plant defence evolution. Trends in Ecology and Evolution. https://doi.org /10.1016/j.tree.2020.12.009

Mertens, D., Bouwmeester, K., and Poelman, E. H. (2020). Variation in insect herbivore communities on individual plants reveals phylogenetic signal in uncertainty of attack in Brassicaceae. PhD thesis chapter 3. 
Mertens, D., Fernández de Bobadilla, M., Rusman, Q., Bloem, J., Douma, J. C., and Poelman, E. H. (2021). Plant defence to sequential attack is adapted to prevalent herbivores. To submitted by Nature Plants.

Mertens, D., Kamps, B., Zhu, Y., Zwartsenberg, S. A., and Poelman, E. H. (2020). Early-season herbivory differentially affects herbivore community assembly and plant reproduction in four closely related Brassicaceae species. PhD thesis chapter 6 .

Moisan, K., Raaijmakers, J. M., Dicke, M., Lucas-Barbosa, D., and Cordovez, V. (2021). Volatiles from soil-borne fungi affect directional growth of roots. Plant Cell and Environment, 44(1), 339-345. https://doi.org/10.1111/ pce. 13890

Moreira, X., Abdala-Roberts, L., Hernandez-Cumplido, J., Cuny, M. A. C., Glauser, G., and Benrey, B. (2015). Specificity of induced defenses, growth, and reproduction in lima bean (Phaseolus lunatus) in response to multispecies herbivory. American Journal of Botany, 102(8), 1300-1308. https://doi.org/10.3732/ajb.1500255

Moreira, X., Abdala-Roberts, L., and Castagneyrol, B. (2018). Interactions between plant defence signalling pathways: Evidence from bioassays with insect herbivores and plant pathogens. Journal of Ecology, 106(6), 2353-2364. https:// doi.org/10.1111/1365-2745.12987

Moreira, X., Castagneyrol, B., Abdala-Roberts, L., and Traveset, A. (2019). A meta-analysis of herbivore effects on plant attractiveness to pollinators. Ecology, 100(6), 1-8. https:// doi.org/10.1002/ecy.2707

Mutti, N. S., Louis, J., Pappan, L. K., Pappan, K., Begum, K., Chen, M. S., Park, Y., Dittmer, N., Marshall, J., Reese, J. C., et al. (2008). A protein from the salivary glands of the pea aphid, Acyrthosiphon pisum, is essential in feeding on a host plant. Proceedings of the National Academy of Sciences of the United States of America, 105(29), 9965-9969. https://doi.org/10.1073/pnas.0708958105

Nabity, P. D., Zavala, J. A., and DeLucia, E. H. (2009). Indirect suppression of photosynthesis on individual leaves by arthropod herbivory. Annals of Botany, 103(4), 655-663. https://doi.org/10.1093/aob/mcn127 
Neilson, E. H., Goodger, J. Q. D., Woodrow, I. E., and Lindberg Møller, B. (2013). Plant chemical defense: at what cost? Trends in Plant Science, 18(5), 250-258. https:// doi.org/10.1016/j.tplants.2013.01.001

Nguyen, D., Rieu, I., Mariani, C., and van Dam, N. M. (2016). How plants handle multiple stresses: hormonal interactions underlying responses to abiotic stress and insect herbivory. Plant Molecular Biology, 91(6), 727-740. https: // doi.org/10.1007/s11103-016-0481-8

Nicholls, E., and Hempel de Ibarra, N. (2017). Assessment of pollen rewards by foraging bees. Functional Ecology, 31(1), 76-87. https:// doi.org/ 10.1111/1365-2435.12778

Ochoa-Lopez, S., Damián, X., Rebollo, R., Fornoni, J., Domínguez, C. A., and Boege, K. (2020). Ontogenetic changes in the targets of natural selection in three plant defenses. New Phytologist, 226(5), 1480-1491. https:// doi. org $/ 10.1111 /$ nph.16422

Ohgushi, T. (2005). Indirect interaction webs: Herbivore-induced effects through trait change in plants. Annual Review of Ecology, Evolution, and Systematics, 36, 80-105. https://doi.org/10.1146/annurev.ecolsys.36.091704.175523

Ohgushi, T. (2008). Herbivore-induced indirect interaction webs on terrestrial plants: The importance of non-trophic, indirect, and facilitative interactions. Entomologia Experimentalis et Applicata, 128(1), 217-229. https://doi.org/10.1111/j.1570-7458.2008.00705.x

Ollerton, J., Winfree, R., and Tarrant, S. (2011). How many flowering plants are pollinated by animals? Oikos, 120(3), 321-326. https://doi.org /10.1111/j.1600-0706.2010.18644.x

Onkokesung, N., Baldwin, I. T., and Gális, I. (2010). The role of jasmonic acid and ethylene crosstalk in direct defense of Nicotiana attenuata plants against chewing herbivores. Plant Signaling \& Behavior, 5(10), 1305-1307. https://doi.org/10.4161/psb.5.10.13124

Onkokesung, N., Reichelt, M., van Doorn, A., Schuurink, R. C., and Dicke, M. (2016). Differential costs of two distinct resistance mechanisms induced by different herbivore species in Arabidopsis. Plant Physiology, 170(2), 891-906. https: / / doi.org/10.1104/pp.15.01780 
Orrock, J. L., Connolly, B. M., Choi, W.-G., Guiden, P. W., Swanson, S. J., and Gilroy, S. (2018). Plants eavesdrop on cues produced by snails and induce costly defenses that affect insect herbivores. Oecologia, 186(3), 703-710. https:// doi.org/10.1007/s00442-018-4070-1

Orrock, J. L., Sih, A., Ferrari, M. C. O., Karban, R., Preisser, E. L., Sheriff, M. J., and Thaler, J. S. (2015). Error management in plant allocation to herbivore defense. Trends in Ecology and Evolution, 30, 441-445. https: // doi.org/10.1016/j.tree.2015.06.005

Pan, G., Liu, Y. Q., Ji, L. S., Zhang, X., He, J., Huang, J., Qiu, Z. Y., Liu, D. M., Sun, Z. G., Xu, T. T., et al. (2018). Brassinosteroids mediate susceptibility to brown planthopper by integrating with the salicylic acid and jasmonic acid pathways in rice. Journal of Experimental Botany, 69(18), 4433-4442. https://doi.org/10.1093/jxb/ery223

Pashalidou, F. G., Frago, E., Griese, E., Poelman, E. H., van Loon, J. J. A., Dicke, M., and Fatouros, N. E. (2015). Early herbivore alert matters: plantmediated effects of egg deposition on higher trophic levels benefit plant fitness. Ecology Letters, 18(9), 927-936. https://doi.org/10.1111/ele.12470

Papazian, S., Girdwood, T., Wessels, B. A., Poelman, E. H., Dicke, M., Moritz, T., and Albrectsen, B. R. (2019). Leaf metabolic signatures induced by real and simulated herbivory in black mustard (Brassica nigra). Metabolomics, 15(10), 1-16. https://doi.org/10.1007/s11306-019-1592-4

Perumal, S., Koh, C. S., Jin, L., Buchwaldt, M., Higgins, E. E., Zheng, C., Sankoff, D., Robinson, S. J., Kagale, S., Navabi, Z. K., Tang, L., Horner, K. N., He, Z., Bancroft, I., Chalhoub, B., Sharpe, A. G., and Parkin, I. A. P. (2020). A high-contiguity Brassica nigra genome localizes active centromeres and defines the ancestral Brassica genome. Nature Plants, 6(8), 929-941. https: //doi.org/10.1038/s41477-020-0735-y

Pieterse, C. M. J., Van der Does, D., Zamioudis, C., Leon-Reyes, A., and Van Wees, S. C. M. (2012). Hormonal Modulation of Plant Immunity. Annual Review of Cell and Developmental Biology, 28(1), 489-521. https://doi.org /10.1146/annurev-cellbio-092910-154055 
Pineda, A., Soler, R., Pastor, V., Li, Y., and Dicke, M. (2017). Plant-mediated species networks: the modulating role of herbivore density. Ecological Entomology, 42(4), 449-457. https://doi.org/10.1111/een.12404

Pineda, A., Zheng, S. J., van Loon, J. J., Pieterse, C. M., and Dicke, M. (2010). Helping plants to deal with insects: the role of beneficial soil-borne microbes. Trends in Plant Science, 15(9), 507-514. https://doi.org /10.1016/j.tplants.2010.05.007

Pinheiro, J., Bates, D., DebRoy, S., and Sarkar, D. (2019). R Core Team, nlme: Linear and Nonlinear Mixed Effects Models. R package version 3.1-141, https: //CRAN.R-project.org/package=nlme.

Poelman, E. H., Broekgaarden, C., Van Loon, J. J. A., and Dicke, M. (2008). Early season herbivore differentially affects plant defence responses to subsequently colonizing herbivores and their abundance in the field. Molecular Ecology, 17(14), 3352-3365. https://doi.org/10.1111/j.1365294X.2008.03838.x

Poelman, E. H., and Kessler, A. (2016). Keystone herbivores and the evolution of plant defenses. Trends in Plant Sciences, 21(6), 477-485. https: / / doi.org /10.1016/j.tplants.2016.01.007

Poelman E. H., Oduor A. M. O., Broekgaarden C., Hordijk C. A., Jansen J. J, Van Loon J. J. A. , Van Dam N. M., Vet L. E. M., and Dicke M. (2009). Field parasitism rates of caterpillars on Brassica oleracea plants are reliably predicted by differential attraction of Cotesia parasitoids. Functional Ecology, 23(5), 951-962. https://doi.org/10.1111/j.13652435.2009.01570.x

Poelman, E. H., Van Dam, N. M., Van Loon, J. J. A., Vet, L. E. M., and Dicke, M. (2009). Chemical diversity in Brassica oleracea affects biodiversity of insect herbivores. Ecology, 9077), 1863-1877. https://doi.org/10.1890/08-0977.1

Poelman E. H., van Loon J. J., and Dicke M. (2008). Consequences of variation in plant defense for biodiversity at higher trophic levels. Trends in Plant Science, 13(10), 534-541. https:// doi.org/10.1016/j.tplants.2008.08.003

Ponzio, C., Papazian, S., Albrectsen, B. R., Dicke, M., and Gols, R. (2017). Dual herbivore attack and herbivore density affect metabolic profiles of 
Brassica nigra leaves. Plant Cell and Environment, 40(8), 1356-1367. https:// doi.org/10.1111/pce.12926

Ponzio, C., Cascone, P., Cusumano, A., Weldegergis, B. T., Fatouros, N. E., Guerrieri, E., Dicke, M., and Gols, R. (2016). Volatile-mediated foraging behaviour of three parasitoid species under conditions of dual insect herbivore attack. Animal Behaviour, 111, 197-206. https://doi.org /10.1016/j.anbehav.2015.10.024

Proietti, S., Caarls, L., Coolen, S., Van Pelt, J. A., Van Wees, S. M., and Pieterse, C. M. J. (2018). Genome-wide association study reveals novel players in defense hormone crosstalk in Arabidopsis. Plant Cell and Environment, 41(10), 2342-2356. https://doi.org/10.1111/pce.13357

Quintana-Rodriguez, E., Rivera-Maciasa, L. E., Adame-Alvareza, R. M., Molina Torres, J., and Heil, M. (2018). Shared weapons in fungus-fungus and fungus-plant interactions? Volatile organic compounds of plant or fungal origin exert direct antifungal activity in vitro. Fungal Ecology, 33, 115-121. https: / / doi.org/10.1016/j.funeco.2018.02.005

R Core Team (2019). R version 3.6.1. R: A language and environment for statistical computing. R Foundation for Statistical Computing. Vienna, Austria.

Ramirez, R. A., and Eubanks, M. D. (2016). Herbivore density mediates the indirect effect of herbivores on plants via induced resistance and apparent competition. Ecosphere, 7(2), e01215. https://doi.org/10.1002/ ecs 2.1218

Ray, S., Gaffor, I., Acevedo, F. E., Helms, A. M., Chuang, W.-P., Tooker, J. F., Felton, G. W., and Luthe, D. S. (2015). Maize plants recognize herbivoreassociated cues from caterpillar frass. Journal of Chemical Ecology, 41(9), 781-792. https: // doi.org/10.1007/s10886-015-0619-1

Rasmann, S., Chassin, E., Bilat, J., Glauser, G., and Reymond, P. (2015). Tradeoff between constitutive and inducible resistance against herbivores is only partially explained by gene expression and glucosinolate production. Journal of Experimental Botany, 66(9), 2527-2534. https://doi.org/10.1093/jxb/erv033

Rodriguez-Saona, C., Chalmers, J. A., Raj, S., and Thaler, J. S. (2005). Induced plant responses to multiple damagers: Differential effects on an herbivore 
and its parasitoid. Oecologia, 143(4), 566-577. https://doi.org/10.1007/ s00442-005-0006-7

Rodriguez-Saona, C., Crafts-Brandner, S. J., and Cañas, L. A. (2003). Volatile emissions triggered by multiple herbivore damage: Beet armyworm and whitefly feeding on cotton plants. Journal of Chemical Ecology, 29(11), 2539-2550. https:// doi.org/10.1023/A:1026314102866

Rodriguez-Saona, C. R., Musser, R. O., Vogel, H., Hum-Musser, S. M., and Thaler, J. S. (2010). Molecular, Biochemical, and Organismal Analyses of Tomato Plants Simultaneously Attacked by Herbivores from Two Feeding Guilds. Journal of Chemical Ecology, 36(10), 1043-1057. https:// doi.org/10.1007/s10886-010-9854-7

Rodriguez-Saona, C., and Thaler, J. S. (2005). The jasmonate pathway alters herbivore feeding behaviour: Consequences for plant defences. Entomologia Experimentalis et Applicata, 115(1), 125-134. https://doi.org /10.1111/j.1570-7458.2005.00277.x

Romero, G. Q., and Koricheva, J. (2011). Contrasting cascade effects of carnivores on plant fitness: A meta-analysis. Journal of Animal Ecology, 80(3), 696-704. https://doi.org/10.1111/j.1365-2656.2011.01808.x

Rosseel, Y. (2012). lavaan: An R Package for Structural Equation Modeling. Journal of Statistical Software, 48(2), 1-36. http://www.jstatsoft.org/v48/i02/

Rosseel, Y. (2020). The lavaan tutorial. Department of Data Analysis: Ghent University.

Rubin, I. N., Ellner, S. P., Kessler, A., and Morrell, K. A. (2015). Informed herbivore movement and interplant communication determine the effects of induced resistance in an individual-based model. Journal of Animal Ecology, 84(5), 1273-1285. https://doi.org/10.1111/1365-2656.12369

Ruan, Y. L., Patrick, J. W., Shabala, S., and Slewinski, T. L. (2013). Uptake and regulation of resource allocation for optimal plant performance and adaptation to stress. Frontiers in Plant Science, 455(4), 455. https://doi.org/ https://doi.org/10.3389/fpls.2013.00455

Rusman, Q., Lucas-Barbosa, D., and Poelman, E. H. (2018). Dealing with mutualists and antagonists: Specificity of plant-mediated interactions 
between herbivores and flower visitors, and consequences for plant fitness. Functional Ecology, 32(4), 1022-1035. https://doi.org /10.1111/1365-2435.13035

Rusman, Q., Lucas-Barbosa, D., Poelman, E. H., and Dicke, M. (2019a). Ecology of Plastic Flowers. Trends in Plant Science, 24(8), 725-740. https://doi.org /10.1016/j.tplants.2019.04.007

Rusman, Q., Poelman, E. H., Nowrin, F., Polder, G., and Lucas-Barbosa, D. (2019b). Floral plasticity: Herbivore-species-specific-induced changes in flower traits with contrasting effects on pollinator visitation. Plant Cell and Environment, 42(6), 1882-1896. https://doi.org/10.1111/pce.13520

Rusman, Q., Lucas-Barbosa, D., Hassan, K., and Poelman, E. H. (2020). Plant ontogeny determines strength and associated plant fitness consequences of plant-mediated interactions between herbivores and flower visitors. Journal of Ecology, 108(3), 1046-1060. https://doi.org/10.1111/13652745.13370

Sanders, D., Sutter, L., and van Veen, F. J. F. (2013). The loss of indirect interactions leads to cascading extinctions of carnivores. Ecology Letters, 16(5), 664-669. https://doi.org/10.1111/ele.12096

Sanders, D., Thébault, E., Kehoe, R., and van Veen, F. J. F. (2018). Trophic redundancy reduces vulnerability to extinction cascades. Proceedings of the National Academy of Sciences of the United States of America, 115(10), 2419-2424. https://doi.org/10.1073/pnas.1716825115

Santamaria, M. E., Garcia, A., Arnaiz, A., Rosa-Diaz, I., Romero-Hernandez, G., Diaz, I., and Martinez, M. (2021). Comparative transcriptomics reveals hidden issues in the plant response to arthropod herbivores. Journal of Integrative Plant Biology, 63(2), 312-326. https://doi.org/10.1111/jipb.13026

Sarde, S. J., Kumar, A., Remme, R. N., and Dicke, M. (2018). Genome-wide identification, classification and expression of lipoxygenase gene family in pepper. Plant Molecular Biology, 98(4-5), 375-387. https://doi.org /10.1007/s11103-018-0785-y

Schlinkert, H., Westphal, C., Clough, Y., László, Z., Ludwig, M., and Tscharntke, T. (2015). Plant size as determinant of species richness of herbivores, 
natural enemies and pollinators across 21 Brassicaceae species. PloS ONE, 10, e0135928. https:// doi.org/10.1371/journal.pone.0135928

Schmelz, E. A., Carroll, M. J., LeClere, S., Phipps, S. M., Meredith, J., Chourey, P. S., Alborn, H. T., and Teal, P. E. A. (2006). Fragments of ATP synthase mediate plant perception of insect attack. Proceedings of the National Academy of Sciences of the United States of America, 103(23), 8894-8899. https://doi.org/10.1073/pnas.0602328103

Schoonhoven, L. M., van Loon, J. J. A., and Dicke, M. (2005). Insect-Plant Biology. Oxford University Press, Oxford.

Schuman, M. C., and Baldwin, I.T. (2016). The layers of plant responses to insect herbivores. Annual Review of Entomology, 61, 373-394. http://doi. org/10.1146/annurev-ento-010715-023851

Schweiger, R., Heise, A. M., Persicke, M., and Müller, C. (2014). Interactions between the jasmonic and salicylic acid pathway modulate the plant metabolome and affect herbivores of different feeding types. Plant, Cell and Environment, 37(7), 1574-1585. https://doi.org/10.1111/pce.12257

Snoeren, T. A. L., Broekgaarden, C., and Dicke, M. (2011). Jasmonates differentially affect interconnected signal-transduction pathways of Pieris rapae-induced defenses in Arabidopsis thaliana. Insect Science, 18, 249-258. https://doi.org/10.1111/j.1744-7917.2010.01364.x

Soler, R., Badenes-Pérez, F. R., Broekgaarden, C., Zheng, S. J., David, A., Boland, W., and Dicke, M. (2012). Plant-mediated facilitation between a leaf-feeding and a phloem-feeding insect in a brassicaceous plant: From insect performance to gene transcription. Functional Ecology, 26(1), 156166. https://doi.org/10.1111/j.1365-2435.2011.01902.x

Soler, R., Bezemer, T. M., Van Der Putten, W. H., Vet, L. E. M., and Harvey, J. A. (2005). Root herbivore effects on above-ground herbivore, parasitoid and hyperparasitoid performance via changes in plant quality. Journal of Animal Ecology, 74(6), 1121-1130. https://doi.org/10.1111/j.13652656.2005.01006.x

Soler, R., Harvey, J. A., Kamp, A. F. D., Vet, L. E. M., Van Der Putten, W. H., Van Dam, N. M., Stuefer, J. F., Gols, R., Hordijk, C. A., and Bezemer, T. 
M. (2007). Root herbivores influence the behaviour of an aboveground parasitoid through changes in plant-volatile signals. Oikos, 116(3), 367376. https://doi.org/10.1111/j.0030-1299.2007.15501.x

Soliveres, S., van der Plas, F., Manning, P., Prati, D., Gossner, M. M. , Renner, S. C., Alt, F., Arndt, H., Baumgartner, V., Bikenstein, J., et al. (2016). Biodiversity at multiple trophic levels is needed for ecosystem multifunctionality. Nature, 536(7617), 456-459. https://doi.org/10.1038/nature19092

Soper Gorden, N. L., and Adler, L. S. (2016). Florivory shapes both leaf and floral interactions. Ecosphere, 7(6), 1-15. https://doi.org/10.1002/ecs2.1326

Soper Gorden, N. L., and Adler, L. S. (2018). Consequences of multiple flowerinsect interactions for subsequent plant-insect interactions and plant reproduction. American Journal of Botany, 105(11), 1835-1846. https:// doi.org/10.1002/ajb2.1182

Stam, J. M., Chrétien, L., Dicke, M., and Poelman, E. H. (2017). Response of Brassica oleracea to temporal variation in attack by two herbivores affects preference and performance of a third herbivore. Ecological Entomology, 42(6), 803-815. https:// doi.org/10.1111/een.12455

Stam, J. M., Dicke, M., and Poelman, E. H. (2018). Order of herbivore arrival on wild cabbage populations influences subsequent arthropod community development. Oikos, 127(10), 1482-1493. https://doi.org/10.1111/oik.05265

Stam, J. M., Kos, M., Dicke, M., and Poelman, E. H. (2019). Cross-seasonal legacy effects of arthropod community on plant fitness in perennial plants. Journal of Ecology, 107(5), 2451-2463. https:// doi.org/10.1111/1365-2745.13231

Stam, J. M., Kroes, A., Li, Y., Gols, R., van Loon, J. J. A., Poelman, E. H., and Dicke, M. (2014). Plant interactions with multiple insect herbivores: from community to genes. Annual Review Plant Biology, 65, 689-713. https: // doi.org/10.1146/annurev-arplant-050213-035937

Stamp, N. (2001). Enemy-free space via host plant chemistry and dispersion: Assessing the influence of tri-trophic interactions. Oecologia, 128(2), 153163. https: / / doi.org/10.1007/s004420100679

Strauss, S. Y. (2014). Ecological and evolutionary responses in complex communities: Implications for invasions and eco-evolutionary 
feedbacks. Oikos, 123(3), 257-266. https://doi.org/10.1111/j.16000706.2013.01093.x

Thaler, J. S., Humphrey, P. T., and Whiteman, N. K. (2012). Evolution of jasmonate and salicylate signal crosstalk. Trends in Plant Science, 17(5), 260-270. https://doi.org/10.1016/j.tplants.2012.02.010

Tiffin, P., Inouye, B. D., and Underwood, N. (2006). Induction and herbivore mobility affect the evolutionary escalation of plant defence. Evolutionary Ecology Research, 8(2), 265-277.

Tholl, D., Chen, F., Petri, J., Gershenzon, J., and Pichersky, E. (2005). Two sesquiterpene synthases are responsible for the complex mixture of sesquiterpenes emitted from Arabidopsis flowers. Plant Journal, 42(5), 757-771. https://doi.org/10.1111/j.1365-313X.2005.02417.x

Traw, M. B., and Dawson, T. E. (2006). Reduced performance of two specialist herbivores (Lepidoptera: Pieridae, Coleoptera: Chrysomelidae) on new leaves of damaged Black Mustard Plants. Environmental Entomology, 31(4), 714-722. https://doi.org/10.1603/0046-225X-31.4.714

Truitt, C. L., Wei, H. X., and Paré, P. W. (2004). A Plasma Membrane Protein from Zea mays Binds with the Herbivore Elicitor Volicitin. Plant Cell, 16(2), 523-532. https://doi.org/10.1105/tpc.017723

Tylianakis, J. M., Didham, R. K., Bascompte, J., and Wardle, D. A. (2008). Global change and species interactions in terrestrial ecosystems. Ecology Letters, 11(12), 1351-1363. https://doi.org/10.1111/j.1461-0248.2008.01250.x

Tylianakis, J. M., Tscharntke, T., and Klein, A. M. (2006). Diversity, ecosystem function, and stability of parasitoid-host interactions across a tropical habitat gradient. Ecology, 87(12), 3047-3057. https://doi.org/ 10.1890/0012-9658(2006)87[3047:DEFASO]2.0.CO;2

Utsumi S., Ando Y., and Miki T. (2010). Linkages among trait-mediated indirect effects: a new framework for the indirect interaction web. Population Ecology, 52(4), 485-497. https://doi.org/10.1007/s10144-010-0237-2

Van Dam, N. M., and Raaijmakers, C. E. (2006). Local and systemic induced responses to cabbage root fly larvae (Delia radicum) in Brassica nigra and $B$. oleracea. Chemoecology, 16(1), 17-24. https://doi.org/10.1007/s00049-005-0323-7 
Van Dam, N. M., Raaijmakers, C. E., and Van Der Putten, W. H. (2005). Root herbivory reduces growth and survival of the shoot feeding specialist Pieris rapae on Brassica nigra. Entomologia Experimentalis et Applicata, 115(1), 161-170. https:// doi.org/10.1111/j.1570-7458.2005.00241.x

Van Wallendael, A., Soltani, A., Emery, N. C., Peixoto, M. M., Olsen, J., and Lowry, D. B. (2019). A molecular view of plant local adaptation: Incorporating stress-response networks. Annual Review of Plant Biology, 70, 559-583. https: //doi.org/10.1146/annurev-arplant-050718-100114

Van Wees, S. C., Van der Ent, S., and Pieterse, C. M. (2008). Plant immune responses triggered by beneficial microbes. Current Opinion in Plant Biology, 11(4), 443-448. https://doi.org/10.1016/j.pbi.2008.05.005

Van Zandt, P. A., and Agrawal, A. A. (2004). Specificity of induced plant responses to specialist herbivores of the common milkweed Asclepias syriaca. Oikos, 104(2), 401-409. https:// doi.org/10.1111/j.0030-1299.2004.12964.x

Verhage, A., Vlaardingerbroek, I., Raaymakers, C., Van Dam, N., Dicke, M., VanWees, S., and Pieterse, C. M. (2011). Rewiring of the jasmonate signaling pathway in Arabidopsis during insect herbivory. Frontiers in Plant Science, 2, 47. https://doi.org/10.3389/fpls.2011.00047

Viswanathan, D. V., Lifchits, O. A., and Thaler, J. S. (2007). Consequences of sequential attack for resistance to herbivores when plants have specific induced responses. Oikos, 116(8), 1389-1399. https://doi.org/ 10.1111/j.2007.0030-1299.15882.x

Viswanathan, D. V., Narwani, A. J. T., and Thaler, J. S. (2005). Specificity in induced plant responses shapes patterns of herbivore occurrence on Solanum dulcamara. Ecology, 86(4), 886-896. https://doi.org/ 10.1890/04-0313

Voelckel, C., and Baldwin, I. T. (2004). Herbivore-induced plant vaccination. Part II. Array-studies reveal the transience of herbivore-specific transcriptional imprints and a distinct imprint from stress combinations. Plant Journal, 38(4), 650-63. doi: 10.1111/j.1365-313X.2004.02077.x.

Vogel, H., Kroymann, J., and Mitchell-Olds, T. (2007). Different transcript patterns in response to specialist and generalist herbivores in the wild 
arabidopsis relative Boechera divaricarpa. PLOS ONE, 2(10), e1081. https: //doi.org/10.1371/journal.pone.0001081

Vos, I. A., Moritz, L., Pieterse, C. M. J., and van Wees, S. (2015). Impact of hormonal crosstalk on plant resistance and fitness under multi-attacker conditions. Frontiers in Plant Science, 6, 639. https: / / doi.org/10.3389/fpls.2015.00639

Walling, L. L. (2000). The myriad plant responses to herbivores. Journal of Plant Growth Regulation, 19(2), 195-216. https://doi.org/10.1007/ s003440000026

Wagner, D. L., Grames, E. M., Forister, M. L., Berenbaum, M. R., and Stopak, D. (2021). Insect decline in the Anthropocene: Death by a thousand cuts. Proceedings of the National Academy of Science of the United States of America, 118(2), 1-10. https://doi.org/10.1073/pnas.2023989118

War, A. R., Paulraj, M. G., Ahmad, T., Buhroo, A. A., Hussain, B., Ignacimuthu, S., and Sharma, H. C. (2012). Mechanisms of plant defense against insect herbivores. Plant Signaling \& Behavior, 7(10), 1306-1320. https: / / doi.org/ $10.4161 /$ psb.21663

Warren, M. S., Maes, D., van Swaay, C. A. M., Goffart, P., Van Dyck, H., Bourn, N. A. D., Wynhoff, I., Hoare, D., and Ellis, S. (2020). The decline of butterflies in Europe: problems, significance, and possible solutions. Proceedings of the National Academy of Science of the United States of America, 118(2), e2002551117. https://doi.org/10.1073/pnas.2002551117

Wasternack, C., and Song, S. S. (2017). Jasmonates: biosynthesis, metabolism, and signaling by proteins activating and repressing transcription. Journal of Experimental Botany, 68(6), 1303-1321. https://doi.org/10.1093/jxb/erw443

Wickham, H. (2016). ggplot2: Elegant Graphics for Data Analysis. SpringerVerlag New York. https://doi.org/10.1007/978-0-387-98141-3

Will T., Tjallingii W. F., Thonnessen A., and van Bel, A. J. E. (2007). Molecular sabotage of plant defense by aphid saliva. Proceedings of the National Academy of Sciences of the United States of America, 104(25), 10536-10541. https: / / doi.org/10.1073/pnas.0703535104

Williams, L., Rodriguez-Saona, C., Pare, P. W., and Crafts-Brandner, S. J. (2005). The piercing-sucking herbivores Lygus hesperus and Nezara viridula induce 
volatile emissions in plants. Archives of Insect Biochemistry and Physiology, 58(2), 84-96. https://doi.org/10.1002/arch.20035

Wise, M. J., and Rausher, M. D. (2013). Evolution of resistance to a multipleherbivore community: genetic correlations, diffuse coevolution, and constraints on the plants response to selection. Evolution, 67(3), 17671779. https://doi.org/10.1111/evo.12061

Wolinska, K. W., and Berens, M. L. (2019). Optimal Defense Theory 2.0: tissuespecific stress defense prioritization as an extra layer of complexity. Communicative \& Integrative Biology, 12(1), 91-95. https://doi.org/ 10.1080/19420889.2019.1625661

Ye, M., Luo, S. M., Xie, J. F., Li, Y. F., Xu, T., Liu, Y., Song, Y. Y., Zhu-Salzman, K., and Zeng, R. S. (2012). Silencing COI1 in rice increases susceptibility to chewing insects and impairs inducible defense. PLoS ONE, 7(4), e36214. https: / / doi.org/10.1371/journal.pone.0036214

Zamioudis, C., and Pieterse, C. M. J. (2012). Modulation of host immunity by beneficial microbes. Molecular Plant-Microbe Interactions, 25(2), 139-150. https://doi.org/10.1094/MPMI-06-11-0179

Zarate, S. I., Kempema, L. A., and Walling, L. L. (2007). Silverleaf whitefly induces salicylic acid defenses and suppresses effectual jasmonic acid defenses. Plant Physiology, 143(2), 866- 875. https://doi.org/10.1104/pp.106.090035 Zeileis, A., and Hothorn, T. (2002). Diagnostic checking in regression relationships. $R$ News $2,7-10$.

Zeng, Y., and Wiens, J. J. (2020). Species interactions have predictable impacts on diversification. Ecology Letters, 24(2), 239-248. https: / / doi.org/10.1111/ ele. 13635

Zhang, P.-J., Zheng, S.-J., van Loon, J. J. A., Boland, W., David, A., Mumm, R., and Dicke, M. (2009). Whiteflies interfere with indirect plant defense against spider mites in Lima bean. Proceedings of the National Academy of Scences of the United States of America, 106(50), 21202-21207. https:// doi. org/10.1073/pnas.0907890106

Zuur, A. F., Ieno, E. N., Walker, N., Saveliev, A. A., and Smith, G. M. (2009). Mixed effects models and extensions in ecology with R. Springer, New York, NY 
Züst, T., and Agrawal, A. A. (2016). Mechanisms and evolution of plant resistance to aphids. Nature Plants, 2(1), 1-9. https://doi.org/10.1038/ nplants.2015.206

Züst, T., Heichinger, C., Grossniklaus, U., Harrington, R., Kliebenstein, D. J., and Turnbull, L. A. (2012). Natural enemies drive geographic variation in plant defenses. Science, 338(6103), 116-119. https://doi.org/10.1126/ science. 1226397 


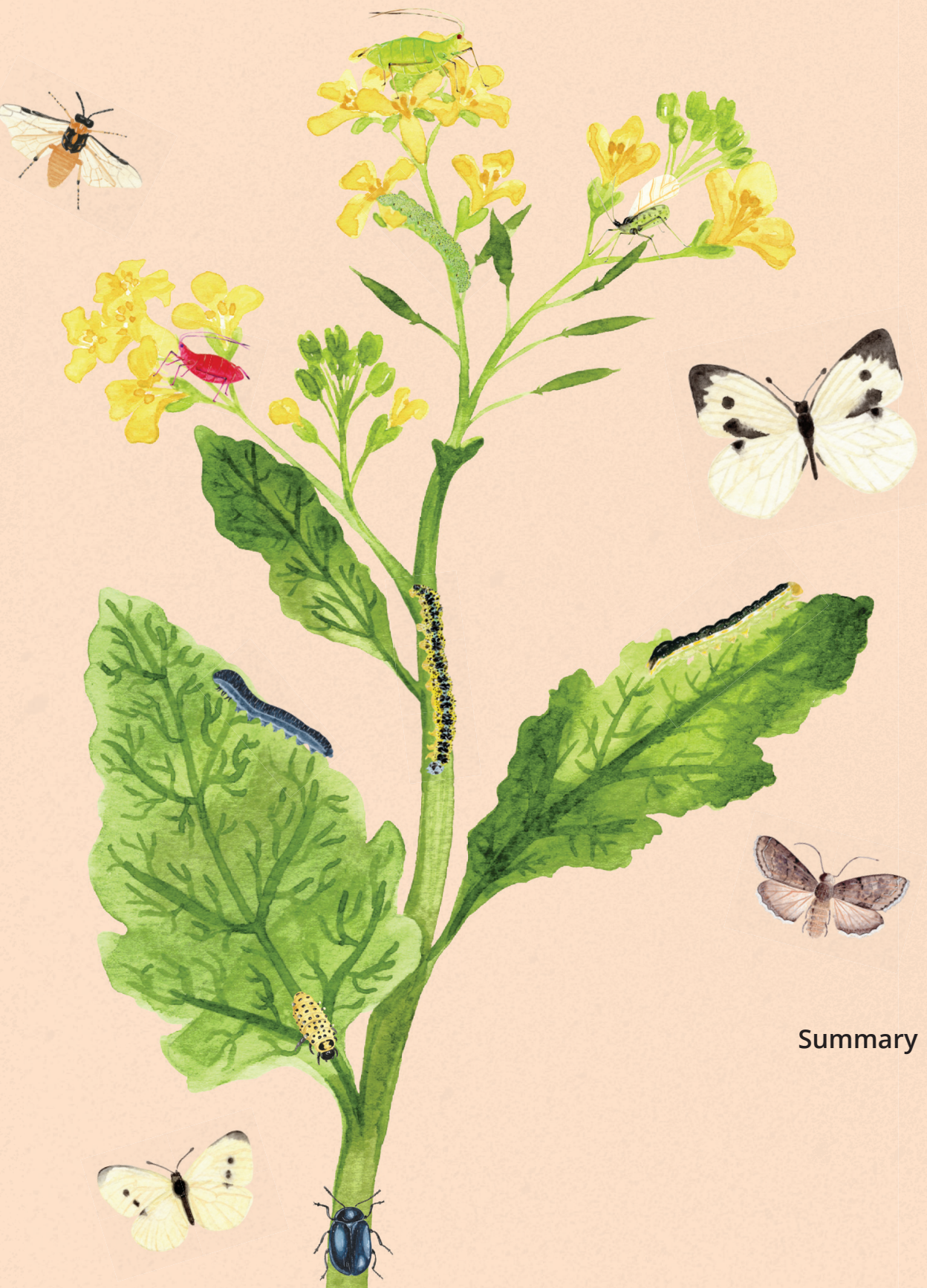

In terrestrial ecosystems, plants are the food source, mating place, or a shelter to hide from predators for many organisms. Consequently, plants are central in many ecosystems and mediate many direct and indirect interactions between organisms. Insects represent one of the most important groups of organisms interacting with plants. It is estimated that there are up to five million species of insects on Earth, of which 50\% are herbivores. Therefore, interactions with insect herbivores are not only very common, but also represent a strong selection force on plant traits. In nature, plants are under the attack by multiple herbivores that arrive simultaneously or sequentially. There is extensive knowledge on plant defence against single or dual attack. However, how plants defend themselves against the attack of multiple insects is poorly understood. Therefore, the aim of my PhD project, was to explore how Brassica nigra plants deal with multi-herbivore attack by its most common herbivores.

Insects represent one of the most diverse groups of organisms, and this diversity is reflected in the array of strategies with which they exploit plants. Plants recognize the attackers by their feeding guild and by elicitors in the saliva and induce a defence response tailor-made to the specific attacker. Although most plants are under attack by a large diversity of herbivores, surprisingly little is known about the physiological capabilities of plants to deal with multiherbivore attack. Studies on dual herbivore attack identified that defence to one attacker may cause energetic and physiological constraints to deal with a second attacker. How these constraints shape plant plasticity in defence to multiherbivore attack and what defence strategies plants may deploy to their full community of attackers is a major knowledge gap in plant science. In chapter 2, I provide a framework for plant defence to multi-herbivore attack by defining the repertoire of plastic defence strategies that may allow plants to optimize defence against a multitude of stressors.

Plants have evolved plastic defence strategies to deal with uncertainty of when, by which species and in which order attack by herbivores will take place. However, due to antagonistic cross-talk between phytohormones, induced plant 
responses to current herbivore attack may come with a cost of compromising resistance to other, later arriving herbivores. Studies on dual attack, show that the feeding guild of the initial herbivore is considered to be the primary factor determining whether resistance to subsequent attack is compromised. In line with the current paradigm of phytohormonal regulation of plant defence to insect herbivory, in chapter 3 I show that plant responses to chewers and phloem feeders are regulated via the SA and JA pathways, respectively. However, also in chapter 3, by investigating 90 pair-wise insect-herbivore interactions among ten different herbivore species, I show that resistance of the annual plant B. nigra to a later arriving herbivore species is not explained by feeding guild of the initial attacker. Instead, the prevalence of herbivore species that arrive on induced plants based on three years of season-long insect community assessments in the field explained cross-resistance. Plants maintained resistance to prevalent herbivores in common patterns of herbivore arrival and compromises in resistance especially occurred for rare patterns of herbivore attack. Therefore, plants tailor induced defence strategies to deal with common patterns of sequential herbivore attack and anticipate arrival of the most prevalent herbivores.

In chapter 4, I went one step further towards more natural conditions, and challenged plants with simultaneous attack by multiple herbivores. I subjected B. nigra plants to 51 treatments representing attack by an increasing species richness (1, 2 or 4 species) of either phloem feeders, leaf chewers, or a mix of both feeding guilds when keeping total density of attackers constant and studied how this affects plant resistance to subsequent attack by caterpillars of the diamondback moth (Plutella xylostella). I show that increased richness in phloem-feeding attackers compromised resistance to P. xylostella. In contrast, leaf chewers induced a stronger resistance to subsequent attack by caterpillars of $P$. xylostella while species richness did not play a significant role for chewing herbivore induced responses. Attack by a mix of herbivores from different feeding guilds resulted in plant resistance similar to resistance levels of plants that were not previously exposed to herbivory. Plant responses to all the chewers 
and to all the combination of chewers were regulated via the JA-pathway. In line with the JA/SA negative crosstalk paradigm I show that feeding by one or by four species of phloem feeders suppressed the expression of a JA-responsive gene compared with undamaged plants, or with plants treated with chewers or with a mix of chewers and phloem feeders. The expression of the SAresponsive gene (PR1) was not induced by any of the phloem feeders, compared to expression levels of these genes in undamaged plants.

In chapter 5, I studied how insect herbivore species richness and trait composition of these species affect plant interactions with other insect community members (i.e. a herbivore, its parasitoids and pollinators) and whether these interactions affect plant fitness. A main challenge in ecology is to assess how loss of species and consequently species interactions affect ecosystem stability. This knowledge is especially relevant within the context of global insect decline that planet Earth faces. I show that herbivore richness, its composition of functional traits and specific herbivore species affected the abundance of P. xylostella, the parasitism rate of $P$. xylostella and pollinator visitation to $B$. nigra plants. The composition of the introduced herbivore community affected plant fitness directly via reducing plant biomass that predicted resource investment into reproductive organs and these effects were stronger than fitness consequences of herbivore-induced plant-mediated interactions with other community members. Fitness effects of indirect interactions often included a network of multiple organisms and could be mediated by plant traits. All of these interactions involved effects of herbivores on pollinator visitation that closely correlated with the total number of seeds produced by a plant. For a species-rich community of phloem feeders as well as for the leaf-chewer rich community seed set was reduced via a chain of interactions that reduced pollinator visitation.

In chapter 4 and in chapter 5 I thus show that the richness of herbivores simultaneously attacking a plant influences a plant's ability to defend itself against a subsequent herbivore and to maintain interactions with mutualistic 
members of the community. However, the question of how plants defend against the attack of multiple herbivores that arrive sequentially, remained unanswered. Therefore, in chapter 6 I investigated whether B. nigra plants are able to defend themselves against caterpillars of the late arriving herbivore $P$. xylostella, when plants had been previously exposed to sequential attack by four other herbivores. I manipulated the order of arrival and the history of attack by four herbivores to investigate which patterns in sequential herbivory determine resistance against the fifth attacker. I found that history of sequential herbivore attack differentially affected the capability of B. nigra plants to defend themselves against larvae of P. xylostella. A sequence of attack with four episodes of attack by $P$. xylostella induced resistance to larvae of P. xylostella. The number of times the plant was attacked by herbivores of the same feeding guild, the identity of the first attacker, the identity and the guild of the last attacker as well as the order of attackers within the sequence of multiple herbivores influenced plant resistance to subsequent herbivory.

In conclusion, my thesis provides answers to important questions on plant responses to multi-herbivore attack. I show that plants tailor induced defence strategies to deal with common patterns of sequential herbivore attack and anticipate arrival of the most prevalent herbivores. Additionally, I show that B. nigra plants channel their defence responses stronger towards a feedingguild specific response when under multi-species attack by herbivores of the same feeding guild, but integrate responses when simultaneously confronted with a mix of herbivores from different feeding guilds. Importantly, under field conditions, I show that herbivore richness, functional trait and herbivore identity affect plant fitness and plant interactions with a herbivore, parasitoids and pollinators. Finally, I show that history of sequential attack is an important factor determining plant resistance to herbivores. 

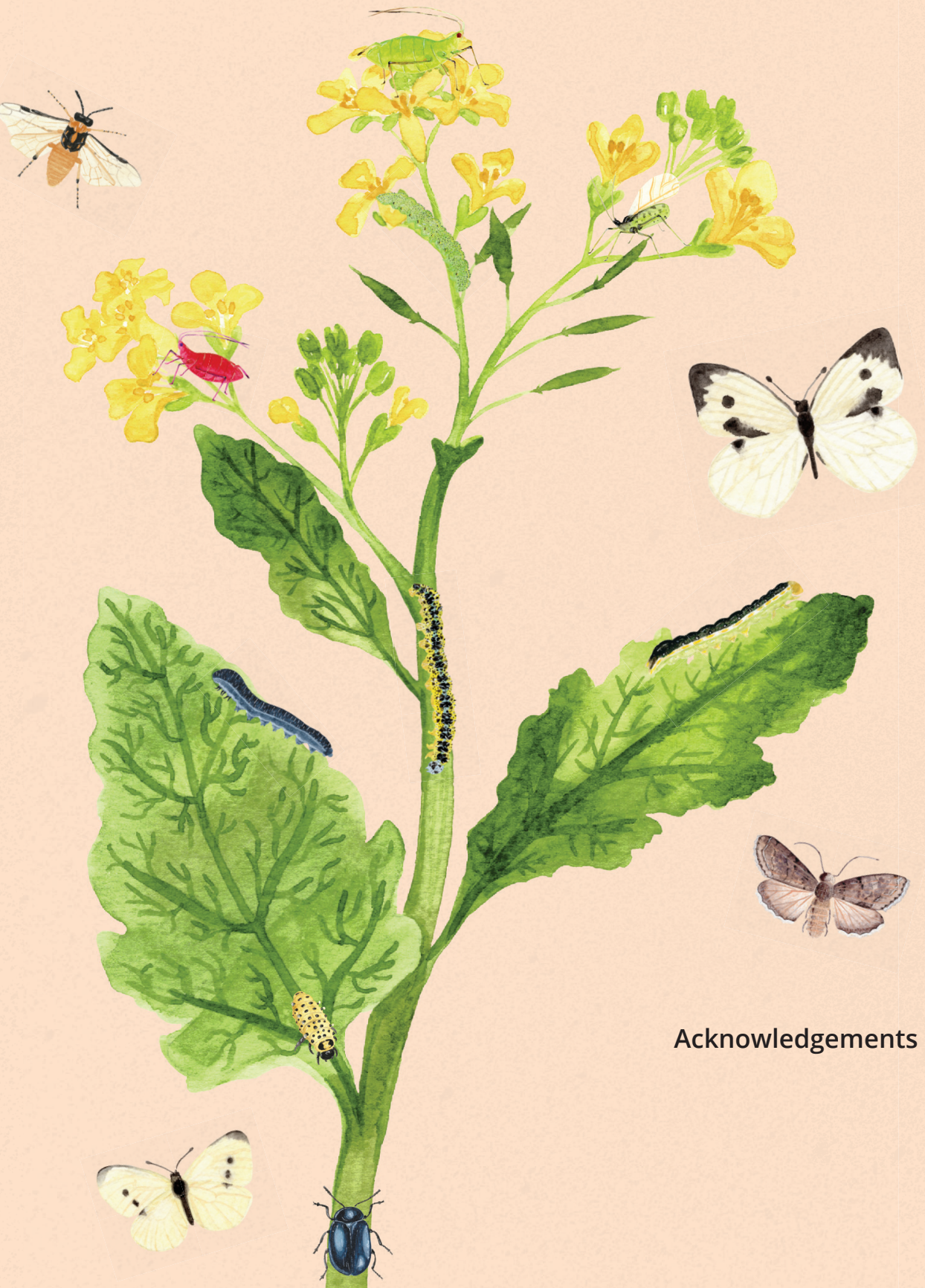

From the 4 years of my $\mathrm{PhD}$ I take many things. I have met great people, had amazing experiences, learnt very useful skills, and I have grown as a person. All this would not have been possible without the contribution of many great persons that I have met on the way. I am very thankful to all of them, and therefore, I would like to dedicate some words to at least the ones that made the biggest impact on my way.

Erik, there are no words to describe how thankful I am to you. I still remember when I met you in Insect Ecology. In your classes I fall in love even more with insects and their interactions. When you offered the $\mathrm{PhD}$ position, I immediately knew I wanted to work in your team. Five years later I can say this was the best decision. You are a very brilliant scientist, but most importantly, you are a great person. You care about people and always find some time in your tight agenda to help with your positive guidance. I take a lot from our interactions, and undoubtfully a way of mentoring I will apply when I teach Biology in Spain. Marcel, I admire your ability to multi-task without compromising excellence. You are a great leader, and a proof of this is the happy, lively and very productive team of the Laboratory of Entomology. Ana, tú despertaste mi interés por la ciencia y me ayudaste a creer en mis capacidades. Transmites tanta alegría y positivismo que después de hablar contigo siento que me puedo comer el mundo. Te has convertido en un referente muy importante en mi vida, a la vez que una amiga.

I would like to continue thanking Erik's team. Daan, thank you for all the great moments working in the greenhouse. Janneke, thank you for your technical assistance especially in the molecular part, but also in the greenhouse. Yavanna, I very much enjoyed the talks with you, as well as your photos and insect outfits. Ilka, I enjoyed our social interactions and your critical input in scientific meetings. Quint, working with you has always been a pleasure. I very much enjoyed our collaborations and I am very thankful for all your help. Alessia, you have been such a great addition to the team, such a hard worker, funny and lovely person! Bram, Luuk, Hanneke, Kyra and Max, thank you 
guys for all the laughs and for the very interesting scientific discussions. Mitchel, a special thanks to you, it was a real pleasure supervising your MSc thesis, and I was super happy you joined the team later.

Antonio, my friend, thank you for all the great moments, I know we will soon meet in Wageningen, Palermo or Valencia. Kathe, mil gracias por todos los bailes y las risas, te estaré esperando en Valencia. Kay, I really admire you in many aspects. It was great sharing many social moments with you, and even better sharing your house. I am super thankful for all your support and your friendship. Lucille, you have been one of my most important referents during the $\mathrm{PhD}$, thank you for all the chats, laughs, rides, music, poetry and art. Sandeep, really thank you for all the deep conversations we had, and the many laughs. Alexander, I really enjoyed talking with you about any topic. Gonzalo, gracias por traer risas, bailes y buenas conversaciones al grupo.

A big thank you to the students who chose my project to do their thesis. Sarah, you were the first student I supervised and I learnt a lot working with you. Roel, it was a pleasure working with you. Onne, I had a great time with you, listening to music and talking about festivals while inducing the plants. Kelly, my dearest camisa negra, you started as a student and ended as a very close friend. I love you, your happiness and your Greek drama. Cris, trabajar contigo fue un placer, y de estudiante pasaste a ser una gran amiga. Te quiero mucho y te deseo lo mejor.

Un especial agradecimiento para mis chicas del aquelarre, Karol y Mile. Mil gracias por todas las risas, los bailes, los chismes y la terapia. Sin duda os habéis convertido en grandes amigas. Tim, you are one of the first persons I met in Wageningen, in Haarweg 185. I always very much enjoyed the interactions with you at the house, at ento, at bootcamp and later during your visits to Valencia. Jeroen Alkema, we had some nice social moments that I hope we can repeat in the future. Gerard, thank you for being so sweet and always being on track of everyone. Joop, talking with you has always inspired me. Angelique, you were always very helpful and nice to me. Hans, you are a 
very talented photographer, as well as an admirable sportsman. Thank you for decorating the lab with your art. Patrick, thank you so much for everything. It was great working with you in the molecular lab, you were always very helpful, with a big smile and great music in the background. I also want to thank Rieta, Helen, Marieke, Pieter, Peter de Jong, Tessa, Davy, Max, Yidong, Filippo, Stijn, Steve, Karen, Jeroen Spitzen, Shaphan, Peter Karssemeijer, Julia, Els, Eveline, Julien, ALex Haverkamp, Gabriella, Jeanine. Thanks to Léon, Frans, André, Pieter, Seppe and Kimmy for culturing the insects.

I would like to thank Unifarm, especially Sean. Thank you for all your support for my experiments, you have always been super helpful, and always with a big smile which made my day. Gerrit, muchas gracias por todo. The collaboration with you has been very productive as well as pleasant. I always enjoyed our meetings. I learned very much from you and I hope you come to Valencia, perhaps on your way to find a Pangolin.

Thibault, we met in 2014, and very soon after we became friends. I have no words to thank you for all your support. For all the chats, walks, coffees, dinners with wine and liquors, dancing salsa, the trips, and even a shared thesis chapter. I love you. I know you are one of those friends that arrived to stay. I will be waiting for you in Valencia. Wadih, shukraan mi hermano. I will meet you in 2041 in Denia. Belen, muchísimas gracias por todo. Hemos compartido muchas cosas y te has convertido en alguien muy importante a quien quiero mantener en mi vida. Gauthier, muchas gracias por las excursiones, las cenas, la salsa, las conversaciones y los viajes. Francesco, you brought light, laughs and happiness to my life. Thousands of thanks to you, to Paula and to the whole family Bourlot, who welcome us every Pascueta as one member of your family. Marcela, desde el principio conectamos mucho, pero nunca imagine el nivel de amistad que construiríamos. Te convertiste en parte de mi familia en Holanda. Te agradezco tantísimas cosas que tendrás que volver pronto a visitarme. Jhon, muchas gracias por todos los bailes, las risas, las conversaciones y la escapada a Cádiz. Irina, querida amiga, muchas gracias por todas las conversaciones, las 
risas, los tés y los bailes. Irving, gracias por tu alegría, tus fiestas, y tu ayuda en la mudanza. Me alegro de que tu y Justé estéis disfrutando el apartamento, y espero que me visitéis pronto en España. Moisés, del año que compartimos me llevé muchos aprendizajes a nivel personal, pero también de la vida y por ello te estoy muy agradecida. Te deseo que todo te vaya muy bien.

Jochem, you are the most empathic person I have ever met. You know exactly what to say or not say to make someone feel better. We had so many great moments, and many more still to come. Paula, es war so schön dich kennen zu lernen. Vielen Dank für alle die Joggen- und Kletternabends, für die Kuchen, die Lasagne und die Gläser Wein. Ich habe dich lieb, und bin sehr dankbar für deine Freundschaft. Chris, die Jahre zusammen waren unglaublich schön und dafür bin ich sehr dankbar. Ich habe so viele Sachen von dir gelernt. Vielen Dank für alle. Ich will auch deine Familie danken. Ludwig und Karola, danke weil ich bei euch wie deine Tochter mich gefühlt habe. Tobie, danke für die Ausflüge und das Lachen, und Natalie, danke für die schöne Momente in Finnland.

Me falta por darle las gracias a mi gente de Valencia. Alba, Lucia, Lorena, David, Dani y Bris, millones de gracias porque con nuestros bailes, risas, conversaciones, viajes y excursiones he sobrellevado el fin de la tesis mucho mejor. Guille, nuestros viajes, fiestas y risas han sido la mejor terapia para los momentos duros. Paula, me has ayudado siempre que lo he necesitado y te estoy muy agradecida por ello. Carol, gracias por tu amistad incondicional, sabes que te quiero y que de viejitas seguiremos con nuestra amistad y nuestras risas. Carla, ¿qué te puedo decir? Hemos vivido tantas cosas. Siempre vamos a estar unidas no importa la distancia o el tiempo que llevemos sin hablar. Te quiero y siempre estarás en mi corazón y en el mechoncito que guardo en la mesita de noche. Alvarito, te considero mi mejor amigo. Siempre que te he necesitado has estado ahí, y eres uno de los mayores referentes en mi vida. Muchas gracias también a la gente del IVIA. Mile, gracias por todos los momentos en Valencia. Miguel, a pesar de no haberme incluido en tus publicaciones querría 
que abramos una empresa de control biológico en Valencia. Jesi, me encantó conocerte, y espero que vuelvas pronto para conocerte más e invitarte a unas croquetas. Paco y Azu, muchas gracias por todas las comidas, cenas, cerves y por los vinos y vermús. Espero que muchos más estén por venir. Angeliki, vivir contigo en Wageningen fue una experiencia genial.

Quiero también agradecer a mi familia por todo su apoyo. Mamá, me has dado mucha fuerza para seguir en los momentos duros. Gracias en especial por todo tu apoyo el último año del doctorado, que he vuelto a casa y me has cuidado mucho. Papá, gracias porque me has educado para ser muy trabajadora y siempre mejorar, y eso me ha ayudado a ser quien soy. Tía Carmen, eres un referente para mí en la vida en muchos aspectos. Gracias a ti y al tío por vuestro apoyo. Muchas gracias a todos mis hermanos, en especial a Bea, por las interminables charlas, y a Arancha, por tu apoyo en la distancia.

Per últim, Carlos, moltes gràcies per tot. Mentre el mon s'afonava nosaltres ens vam enamorar. Has contribuït molt a l'èxit del meu doctorat, amb el teu suport pràctic, emocional i intel·lectual. Els últims mesos han sigut meravellosos i tinc moltes ganes de continuar construint amb tu. 


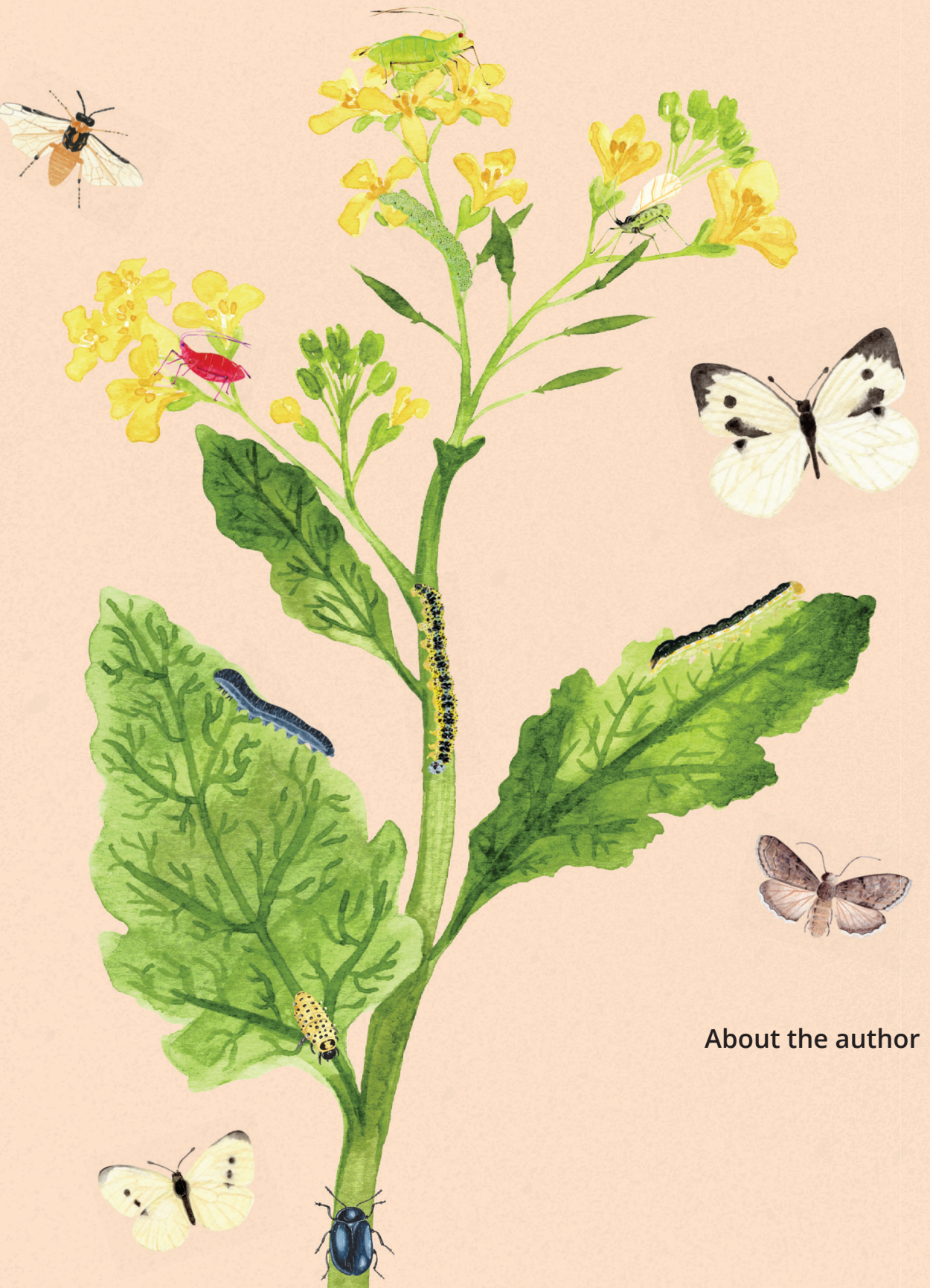

Maite Fernández de Bobadilla was born on July 21st, 1989 in Madrid, Spain. She grew up in Dénia, a very nice town at Alicante's coast. She studied Biology at València. The last year of her bachelor she joined an Erasmus program and moved to Prague. At that moment, she did not know that she would have to wait 8 years to come back to Spain. She lived one year in Augsburg, then moved to Wageningen to join the MSc program Plant Sciences with a focus on Entomology. For her first MSc thesis, supervised by Ana Pineda, she studied the role of drought on the interactions between plants, beneficial bacteria and an insect herbivore. She enjoyed this project so much that she decided doing a second MSc thesis, instead of an internship. She went to the Max Planck Institute in Jena, Germany. There, supervised by Dr Sybille Unsicker and Dr Franziska Eberl, she studied the interactions between poplar trees, a rust fungus and herbivore insects. This thesis was awarded with the East-West Seed Graduation prize for Plant Sciences. Her MSc program was awarded with the qualification of cum laude. In January 2017, she came back to Wageningen to join the team of Dr Erik Poelman as a $\mathrm{PhD}$ candidate. In her $\mathrm{PhD}$ project she studied plant adaptations to the attack of multiple insects. Maite did very diverse experiments, including greenhouse and molecular studies, behavioural assays and field work. The last year of her $\mathrm{PhD}$ Maite joined a training program to become a Biology teacher in Spanish secondary schools. At the beginning of March 2020, Maite came to València to do the practical and teach at a school. She was trapped in Spain due to the Covid-19 pandemic. Maite finished her training program and her $\mathrm{PhD}$ working from home, in València. For the future Maite aims at becoming a Biology teacher in Spain, with a strong emphasis on environmental awareness.

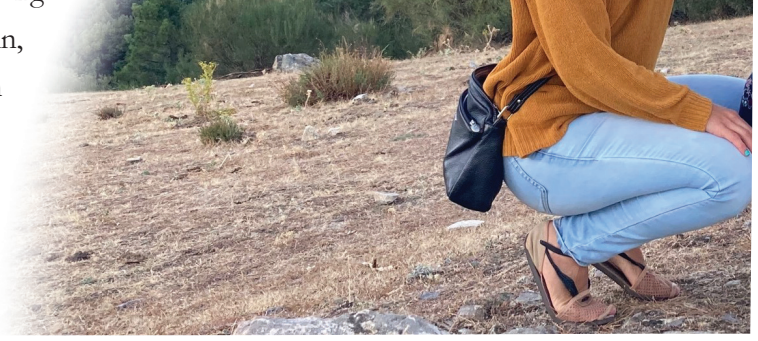




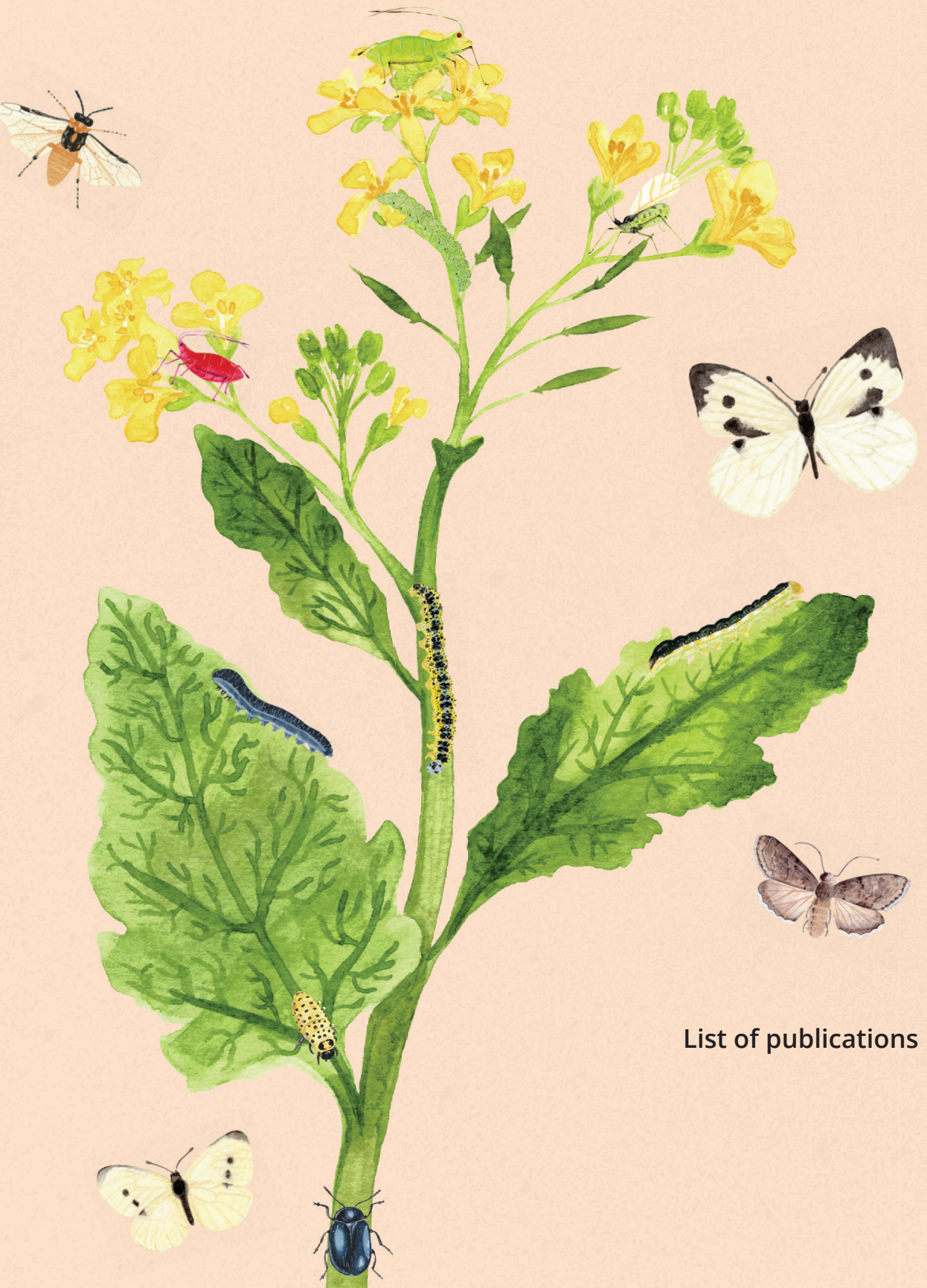



\section{PUBLISHED PAPERS}

Fernández de Bobadilla, M., Bourne, M. E., Bloem, J., Kalisvaart, S. N., Gort, G., Dicke, M., \& Poelman, E. H. (2021). Insect species richness affects plant responses to multi-herbivore attack. New Phytologist. https://doi. org/https://doi.org/10.1111/nph.17228

Eberl, F., Fernandez de Bobadilla, M., Reichelt, M., Hammerbacher, A., Gershenzon, J., \& Unsicker, S. B. (2020). Herbivory meets fungivory: insect herbivores feed on plant pathogenic fungi for their own benefit. Ecology Letters, 23(7), 1073-1084. https://doi.org/10.1111/ele.13506

Aartsma, Y., Cusumano, A., Fernández de Bobadilla, M., Rusman, Q., Vosteen, I., \& Poelman, E. H. (2019). Understanding insect foraging in complex habitats by comparing trophic levels: insights from specialist host-parasitoid-hyperparasitoid systems. Current Opinion in Insect Science, 32, 54-60. https://doi.org/10.1016/j.cois.2018.11.001

Fernández de Bobadilla, M., Friman, J., Pangesti, N., Dicke, M., van Loon, J. J. A., \& Pineda, A. (2017). Does drought stress modify the effects of plantgrowth promoting rhizobacteria on an aboveground chewing herbivore? Insect Science, 24(6), 1034-1044. https://doi.org/10.1111/1744-7917.12477

\section{SUBMITTED PAPERS}

Mertens, D., Fernández de Bobadilla, M., Rusman, Q., Bloem, J., Douma, J. C., \& Poelman, E. H. Plant defence to sequential attack is adapted to prevalent herbivores. Nature Plants.

Fernández de Bobadilla, M., Roel Van Wiechen. Gort, G., \& Poelman, E. H. Plasticity in induced resistance to sequential attack by multiple herbivores in Brassica nigra. Oecologia.

\section{IN PREPARATION}

Fernández de Bobadilla, M., Rusman, Q., Costaz, T., Alban, C., Arkoumanea, K., Mertens, D., \& Poelman, E. H. Herbivore species richness influences plant interactions with an herbivorous insect, its natural enemies, and plant reproduction. Ecology Letters. 


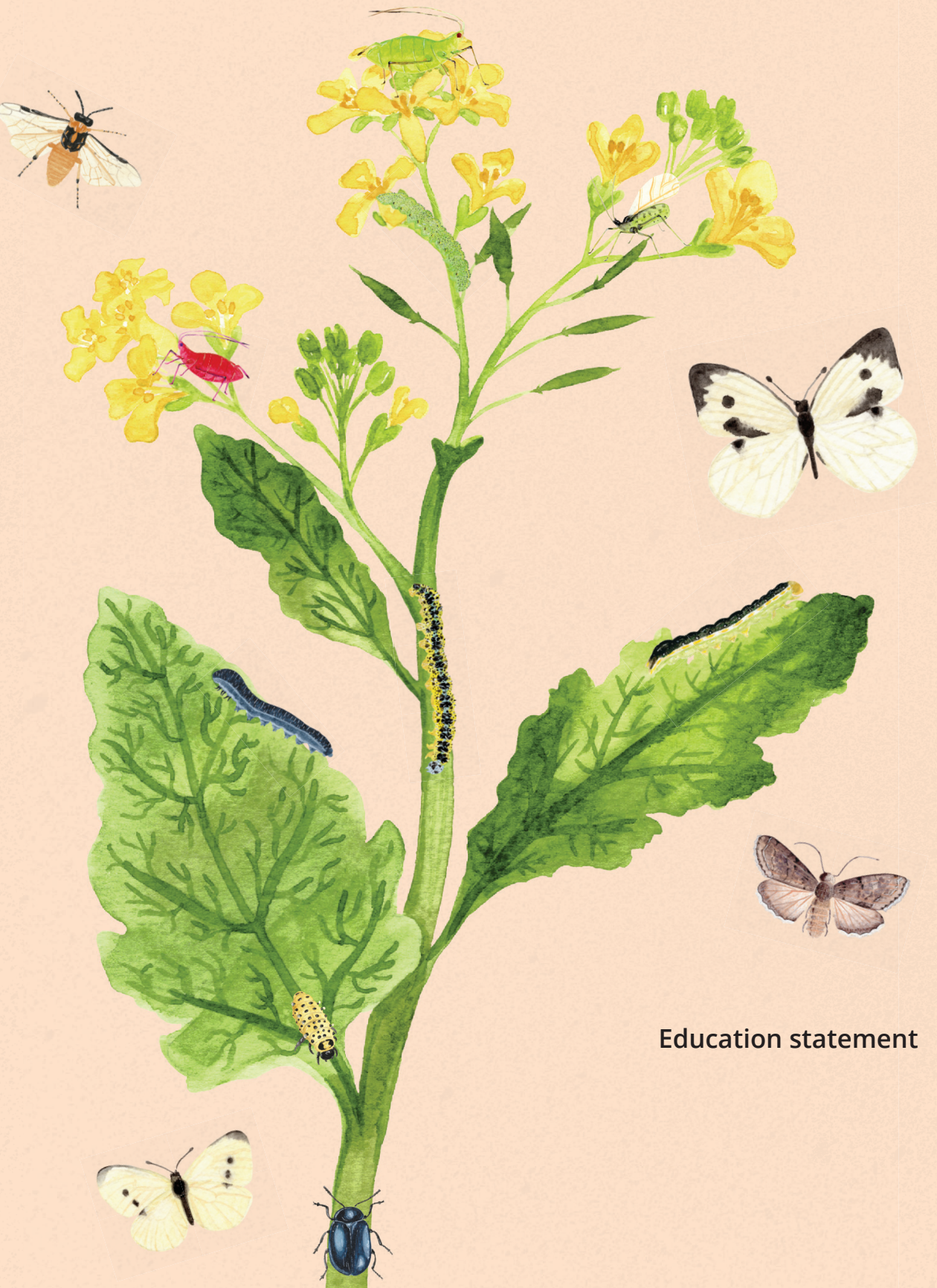



\section{Education Statement of the Graduate School Experimental Plant Sciences}

Issued to: Maite Fernández de Bobadilla

Date:

30 June 2021

Group: Laboratory of Entomology

University: Wageningen University \& Research

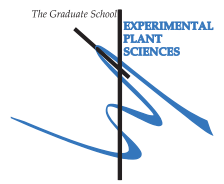

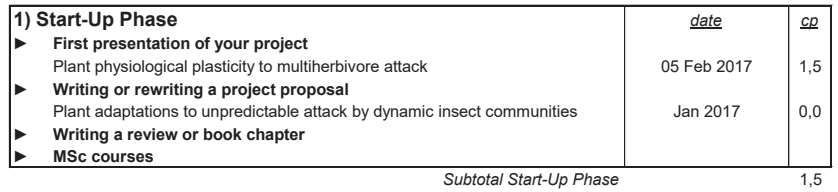

2) Scientific Exposure

- EPS PhD student days

Annual EPS PhD Student Days Get2Gether, Soest, NL

Annual EPS PhD Student Days Get2Gether, Soest, NL

- EPS theme symposia

EPS theme 2 symposium 'Interactions between Plants and Biotic Agents', together with Willie Commelin Scholten Day, Wageningen, NL

EPS theme 2 symposium 'Interactions between Plants and Biotic Agents',

together with Willie Commelin Scholten Day, Amsterdam, NL

EPS theme 2 symposium 'Interactions between Plants and Biotic Agents', together with Willie Commelin Scholten Day, Wageningen, NL

1

Lunteren Days and other national platforms

Netherlands Annual Ecology Meeting (NAEM), Lunteren, NL

29e Entomologendag 2017, Ede, NL

31e Entomologendag 2019, Ede, NL

- Seminars (series), workshops and symposia

4th Annual Wageningen PhD symposium - Science: From local to global, Wag

Insect-Plant-Interaction workshop, Wageningen, NL

Insect-Plant-Interaction workshop, Leiden, NL

Towards Ecological Intensification, the Importance of Biodiversity in

Agricultural Landscapes

Yearly Entomology Research Exchange Meeting (YELREM), Bennekom, NL

Yearly Entomology Research Exchange Meeting (YELREM), Bennekom, NL

Yearly Entomology Research Exchange Meeting (YELREM), Bennekom, NL

WEES seminar: Prof. dr. Bregje Wertheim Evolving immunity "Genomic basis

of the evolution and variation in parasitoid resistance"

WEES seminar: Dr. Mart Krupovic "Natural history of viral capsids"

WEES seminar: Prof. Michael Singer "Host shift in butterflies"

WEES seminar: Prof. Anne-Nathalie Volkoff "Endogenous viruses used by

parasitic wasps to deliver virulence molecules to their hosts"

WEES seminar: Alejandro Tena "Honeydew as food source for insects: a

sweet that can become toxic"

WEES seminar: Fred de Boer "Spatial aspects in disease ecology"

$\underline{\text { date }}$

09-10 Feb 2017

15-16 Feb 2018

$\underline{c p}$

Seminar plus

- International symposia and congresses

16th Symposium on Insect-Plant Interactions, Tours, FR

Spanish Applied Entomology Congress (SEEA), Madrid, ES

8th IOBC Working Group Meeting "Landscape management for functional biod

23 Jan 2017

0,6

\begin{tabular}{l|l}
0,6 \\
\hline
\end{tabular}

24 Jan 2018

0,3

01 Feb 2019

14-15 Feb 2017

15 Dec 2017

13 Dec 2019

03 May 2017

07 Nov 2017

09 Oct 2018

28 Aug 2019

01 Jun 2017

24 May 2018

04 Jun 2019

19 Jan 2017

23 Feb 2017

04 Dec 2017

14 Dec 2017

30 Jan 2018

Presentations

Netherlands Ecological Annual Meeting 2017 (poster presentation)

16th Symposium on Insect-Plant Interactions (poster presentation)

Speaker at IVIA (Valencian Institute of Agrarian Research)

Speaker at SEEA (Spanish Applied Entomology Congress)

Speaker at 31e Entomologendag

26 Feb 2018

02-06 Jul 2017

$04-08$ Nov 2019

27-29 Mar 2019

15 Feb 2017

04 Jul 2017

13 Nov 2018

04 Nov 2019

13 Dec 2019

IAB interview

- Excursions 


\section{3) In-Depth Studies}

- Advanced scientific courses \& workshops

Chemical Ecology, Jena, DE

Generalized Linear Models, Wageningen, NL

Bugs at your service, Wageningen, NL

- Journal club

Entomology, Wageningen, NL

- Individual research training

\begin{tabular}{c|c|}
$\underline{\text { date }}$ & $\underline{c p}$ \\
27 Jun - 08 Jul 2016 & 3,0 \\
14-15 Jun 2018 & 0,6 \\
31 Mar - 5 Apr 2019 & 1,5 \\
$2017-2021$ & 3,0 \\
\hline
\end{tabular}

\section{4) Personal Development}

\section{- General skill training courses}

PhD Competence Assessment, Wageningen, NL

Scientific Artwork with Photoshop and Illustrator, Wageningen, NL

EPS PhD Introduction Course, Wageningen, NL

Scientific Writing, Wageningen, NL

Máster Universitario en Formación del profesorado de Educación Secundaria

Obligatoria y Bachillerato, Formación profesional y Enseñanzas de idiomas,

especialidad Biología y Geología (UNIR) (Master for becoming a Teacher from

Compulsory Secondary Education and Bachiller, Vocational Training and

Language Teaching)

- Organisation of meetings, PhD courses or outreach activities

Membership of EPS PhD Council

Subtotal Personal Development

\begin{tabular}{|c|c|}
$\underline{\text { date }}$ & $\underline{c p}$ \\
Apr 2017 & 0,3 \\
28-29 Apr 2017 & 0,6 \\
26 Sep 2017 & 0,3 \\
02 Sep - 24 Oct 2019 & 1,8 \\
Sep 2019 - Jul 2020 & 60,0 \\
& \\
& \\
&
\end{tabular}

TOTAL NUMBER OF CREDIT POINTS*

Herewith the Graduate School declares that the PhD candidate has complied with the educational requirements set by the Educational Committee of EPS with a minimum total of 30 ECTS credits.

* A credit represents a normative study load of 28 hours of study. 

The research presented in this thesis was performed at the Laboratory of Entomology of Wageningen University \& Research (WUR) and was supported by the European Research Council (ERC) under the European Union's Horizon 2020 research and innovation program (Grant agreement No. 677139 to Erik H. Poelman).

Cover illustrations by Maite Méndez-Bushell

Cover design and thesis layout by Maite Fernández de Bobadilla and Claudia Herrero

Typesetting edition by Blanca Moncunill Solé

Printed by GVO drukkers en vormgevers B.V., Ede, The Netherlands 


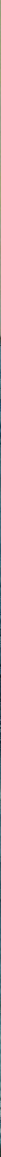

